Florida International University

FIU Digital Commons

FIU Electronic Theses and Dissertations

University Graduate School

$11-24-2004$

\title{
Comparison of coronary heart disease risk factors in foreign-born and United States-born Afro Caribbean Americans and African Americans in South Florida
}

Errol Earl Davis

Florida International University

DOI: $10.25148 /$ etd.FI14062211

Follow this and additional works at: https://digitalcommons.fiu.edu/etd

Part of the Dietetics and Clinical Nutrition Commons

\section{Recommended Citation}

Davis, Errol Earl, "Comparison of coronary heart disease risk factors in foreign-born and United States-born Afro Caribbean Americans and African Americans in South Florida" (2004). FIU Electronic Theses and Dissertations. 2728.

https://digitalcommons.fiu.edu/etd/2728

This work is brought to you for free and open access by the University Graduate School at FIU Digital Commons. It has been accepted for inclusion in FIU Electronic Theses and Dissertations by an authorized administrator of FIU Digital Commons. For more information, please contact dcc@fiu.edu. 
FLORIDA INTERNATIONAL UNIVERSITY

Miami, Florida

COMPARISON OF CORONARY HEART DISEASE RISK FACTORS IN FOREIGNBORN AND UNITED STATES-BORN AFRO CARIBBEAN AMERICANS AND AFRICAN AMERICANS IN SOUTH FLORIDA

A dissertation submitted in partial fulfillment of the requirements for the degree of DOCTOR OF PHILOSOPHY in DIETETICS AND NUTRITION

by

Errol Earl Davis 
To: Dean Ronald M. Berkman

College of Health and Urban Affairs

This dissertation, written by Errol Earl Davis, and entitled Comparison of Coronary Heart Disease Risk Factors in Foreign-born and United States-born Afro Caribbean Americans and African Americans in South Florida, having been approved in respect to style and intellectual content, is referred to you for judgment.

We have read this dissertation and recommend that it be approved.

Michele Ciccazzo

Paulette Johnson

John Landrum

Fatma G. Huffman, Major Professor

Date of Defense: November 24, 2004

The dissertation of Errol Earl Davis is approved.

Dean Ronald M. Berkman College of Health and Urban Affairs

Dean Douglas Wartzok University Graduate School

Florida International University, 2004 
(C) Copyright 2004 by Errol Earl Davis

All rights reserved. 


\section{DEDICATION}

I dedicate this dissertation to my daughter Sofi, sons Damany and Matigani and my parents. Without their love, support, patience and understanding the completion of this project would not have been possible. 


\section{ACKNOWLEDGMENTS}

This dissertation was supported in part by the Florida Foundation Fund. I would like to thank the members of this organization for their kind support. I would like to thank the members of my dissertation committee, Dr. Michele Ciccazzo, Dr. Paulette Johnson and Dr. John Landrum for their valuable suggestions and guidance. A special thank you to my major professor, Dr. Fatma G. Huffman who believed in me. Her advice, commitment, dedication, understanding, encouragement and support during this process were invaluable. Dr. Huffman, without your support and love, this would not have been possible.

I would like to thank all the Florida International University students who participated in this project. A special thank you to my family, friends and colleagues, especially Subrata Nath who helped with several activities related to the data collection process, Dr. Himburg, Dr. Maitland, brothers Albert, Cecil, Dennis, Jeffery, Noel, sister Beverly, cousin Marlene, very special friends Andrea, Bridgette, Chillean, Claudette, Mrs. Pryce, Salomie, Sonio and Tracy for your financial and social support. To Dianne who has been there for me, a very special 'thank you.' It was fun.

Finally, my experience at Florida International University could not have been possible without the power of God. I thank my spiritual father for his guidance, love and blessings throughout this dissertation process. 


\section{ABSTRACT OF THE DISSERTATION \\ COMPARISON OF CORONARY HEART DISEASE RISK FACTORS IN FOREIGN- BORN AND UNITED STATES-BORN AFRO CARIBBEAN AMERICANS AND AFRICAN AMERICANS IN SOUTH FLORIDA \\ by}

Errol Earl Davis

Florida International University, 2004

Miami, Florida

Professor Fatma G. Huffman, Major Professor

In a cross-sectional study design, risk factors for coronary heart disease (CHD) were evaluated in three groups: 66 Afro Caribbeans (FBCA) living in the US for less than 10 years, 62 US-born Afro Caribbean (USBCA) and 61 African American (AA) adults (18-40 years), with equal numbers of males and females in each group. Sociodemographic, dietary, anthropometric and blood pressure data were collected. Fasting blood glucose, blood lipids and high-sensitivity C-reactive protein (hs-CRP) were determined.

The USBCA and AA participants compared to the FBCA participants consumed significantly $(\mathrm{p}<0.05)$ more mean total fat $(\mathrm{g})(66.3 \pm 41.7$ and $73.0 \pm 47.8$ vs. $52.8 \pm$ $32.3)$, saturated fat $(\mathrm{g})(23.1 \pm 14.9$ and $24.9 \pm 15.8$ vs. $18.6 \pm 11.5)$, percent energy from fat $(\%)(33.1 \pm 6.5$ and $31.4 \pm 6.4$ vs. $29.3 \pm 6.8)$, fat servings $(1.8 \pm 1.2$ and $1.5 \pm 1.0$ vs $1.2 \pm 0.9)$, dietary cholesterol $(\mathrm{mg})(220.4 \pm 161.9$ and $244.1 \pm 155.0$ vs. $168.8 \pm 114.0)$ and sodium (mg) (2245.2 \pm 1238.3 and $2402.6 \pm 1359.3$ vs. $1838.0 \pm 983.4)$ and less than 2 servings of fruits per day (\%) (86.9 and 94.9 vs. 78.5$)$. These differences were more 
pronounced in males compared to females and remained after correcting for age. Also, the percentages of USBCA and AA participants who were obese $(17.1 \%$ and $23.0 \%$, respectively) were significantly $(p<0.05)$ higher compared to FBCA $(7.6 \%)$ participants. More USBCA and AA than FBCA individuals smoked cigarettes $(4.8 \%$ and $6.6 \%$ vs. $0.0 \%)$ and consumed alcoholic beverages ( $29.0 \%$ and $50.8 \%$ vs. $24.2 \%)$. The mean hsCRP level of the AA participants $(2.2 \pm 2.7 \mathrm{mg} / \mathrm{L})$ was significantly $(\mathrm{p}<0.01)$ higher compared to the FBCA $(1.1 \pm 1.3 \mathrm{mg} / \mathrm{L})$ and USBCA $(1.3 \pm 1.6 \mathrm{mg} / \mathrm{L})$ participants.

The FBCA participants had a better CHD risk profile than the USBCA and AA participants. Focus should be placed on the ethnic and cultural differences in a population to better understand the variations in health indicators among different ethnic groups of the same race. This focus can provide healthcare professionals and policy planners with the opportunity to develop culturally sensitive programs and strategies for the improvement of health outcomes. 


\section{TABLE OF CONTENTS}

CHAPTER

PAGE

I. INTRODUCTION 1

Statement of the Problem $\quad 2$

Significance 4

Research Questions $\quad 6$

$\begin{array}{ll}\text { Purpose and Specific Objectives } & 7\end{array}$

II. LITERATURE REVIEW 11

Cardiovascular Disease and Coronary Heart Disease $\quad 11$

Culture, Migration, Ethnicity and CHD 15

Race/Ethnic Disparities in Cardiovascular Health 19

African Americans, Foreign-born and US-born Afro

Caribbean Americans $\quad 20$

Risk Factors for CHD $\quad 25$

Socio-demographic Variables $\quad 26$

Age $\quad 26$

Gender $\quad 26$

Socio-economic Status (SES) and Stress 27

Knowledge of Risk Factors for CHD 28

Family History of Premature CHD 29

Behavioral/Environmental Risk Factors for CHD 31

Atherogenic Diet $\quad 31$

African Americans and Afro Caribbeans Eating Habits 34

Physical Activity/Inactivity $\quad 35$

Body Weight and Obesity 36

Cigarette Smoking and Alcohol Consumption $\quad 38$

Bio-physiological Risk Factors for CHD $\quad 40$

Blood Pressure and Hypertension $\quad 40$

Fasting Blood Glucose, Metabolic Syndrome and Diabetes $\quad 42$

Lipid Risk Factors - Serum Lipids $\quad 46$

$\begin{array}{ll}\text { Total Cholesterol } & 46\end{array}$

Low-density Lipoprotein Cholesterol 48

Serum Triglycerides $\quad 49$

High-density Lipoprotein Cholesterol $\quad 49$

Emerging Biochemical Risk Factors for CHD 51

High-sensitivity C-reactive Protein and Coronary Heart Disease 57

Factors that can Influence C-reactive Protein Levels 62

Total Cholesterol/High-density Lipoprotein Cholesterol Ratio 64

Improving the Health of Caribbean Americans 66 
III. METHODOLGY

Study Design

Study Population

Recruitment of Subjects

Data Collection

Statistical Analysis

IV. RESULTS

Section 1 - Research Question 1

Section 2 - Research Question 2

Section 3 - Research Question 3

Section 4 - Research Question 4

Additional Findings

Section 5 - Correlations

Research Questions Summary

V. DISCUSSION

Research Question 1

Research Question 2

Research Question 3

Research Question 4

Additional Findings

198

Strengths of Study

Limitations of the Study

203

Summary

204

Conclusion and Recommendations

\section{APPENDICES}


1 Composition and Growth of the non-Hispanic Black

Populations of the US, $1990-2000$

2 Socio-economic Characteristics of the Non-Hispanic Black Populations in Comparison with Other US Racial and Ethnic Groups, 1990 - 2000

3 Population Attributable Risk Percent for CHD and Stroke

4 Classification of Blood Pressure for Adults Aged 18 Years and Older

5 Clinical Identification of the Metabolic Syndrome

6 NCEP: ATP III Classification of Total Cholesterol, Low-density Lipoprotein Cholesterol, Triglycide and High-density Lipoprotein Cholesterol

$7 \quad$ Emerging Risk Factors for Coronary Heart Disease

8 Sample Size for the Study Population

9 Socio-demographic Factors of Study Participants by Gender

10 Socio-demographic Factors of the Participants by Ethnicity 91

11 Socio-demographic Factors of the Male Participants by Ethnicity

12 Socio-demographic Factors of the Female Participants by Ethnicity

13 Mean Perceived Stress and Knowledge of Coronary Heart Disease Risk Factor Scores of the Participants by Gender, Ethnicity and Gender-ethnicity Subgroups

14 Mean Energy and Nutrient Intakes of the Participants by Gender

15 Mean Energy and Nutrient Intakes of the Participants by Ethnicity 98

16 Mean Energy and Nutrient Intakes of Males by Ethnicity 100

17 Mean Energy and Nutrient Intakes of Females by Ethnicity 101

18 Mean Mineral Intakes of the Participants by Gender 103 
19 Mean Mineral Intakes of the Participants by Ethnicity 104

20 Mean Mineral Intakes of the Male Participants by Ethnicity 105

21 Mean Mineral Intakes of the Female Participants by Ethnicity 106

22 Mean Vitamin Intakes of the Participants by Gender 108

23 Mean Vitamin Intakes of the Participants by Ethnicity 109

24 Mean Vitamin Intakes of the Male Participants by Ethnicity 111

25 Mean Vitamin Intakes of the Female Participants by Ethnicity 112

26 Mean Carotenoid Intakes of the Participants by Gender 113

27 Mean Carotenoid Intakes of the Participants by Ethnicity 114

28 Mean Carotenoid Intakes of the Participants by Gender within Ethnicity 115

29 Macronutrient Intakes of the Participants Compared with

NHANES III by Gender, Ethnicity and Gender-ethnicity Subgroups

30 Percentages of the Participants Consuming Below or Above the

Dietary Guidelines and Food Pyramid Servings of Selected

Macronutrients by Gender

31 Percentages of the Participants Consuming Below or Above the Dietary Guidelines and Food Pyramid Servings of Selected Macronutrients by Ethnicity

32 Percentages of the Male Participants Consuming Below or Above the Dietary Guidelines and Food Pyramid Servings of Selected Macronutrients by Ethnicity

33 Percentages of the Female Participants Consuming Below or Above the Dietary Guidelines and Food Pyramid Servings of Selected Macronutrients by Ethnicity

34 Mean and Frequency Distribution of the Diet Scores of Participants by Gender, Ethnicity and Gender-ethnicity Subgroups

35 Mean Physical Activity Indices of the Participants by Gender, Ethnicity and Gender-ethnicity Subgroups 
36 Distribution of the Physical Activities of the Participants by Gender, Ethnicity and Gender-ethnicity Subgroups

37 Anthropometrics of the Participants by Gender, Ethnicity and Gender-ethnicity Subgroups

38 Distribution of the Anthropometrics of the Participants by Gender, Ethnicity and Gender-ethnicity Subgroups

39 Distribution of Cigarette Smoking and Alcohol Consumption of the Participants by Gender, Ethnicity and Gender-ethnicity Subgroups

40 Mean Blood Pressure Levels of Participants by Gender, Ethnicity and Gender-ethnicity Subgroups

41 Distribution of Blood Pressures of the Participants by Gender, Ethnicity and Gender-ethnicity Subgroups

42 Mean Physiological Risk Factors of the Participants by Gender, Ethnicity and Gender-ethnicity Subgroups

43 Distribution of Physiological Risk Factors of the Participants by Gender

44 Distribution of Physiological Risk Factors of the Participants by Ethnicity

45 Distribution of Physiological Risk Factors of the Male Participants by Ethnicity

46 Distribution of Physiological Risk Factors of the Female Participants by Ethnicity

47 Mean Emerging Risk Factors of the Participants by Gender, Ethnicity and Gender-ethnicity Subgroups

48 Distribution of the Emerging Risk Factors of the Participants by Gender, Ethnicity and Gender-ethnicity Subgroups

49 Mean and Frequency Distribution of the Behavioral/Environmental Risk Factors of Participants by Gender, Ethnicity and Gender-ethnicity Subgroups

50 Risk Factor Categories for the Metabolic Syndrome of Participants by Gender, Ethnicity and Gender-ethnicity Subgroups 
51 Risk Factor Categories for the Metabolic Syndrome of Participants by Gender, Ethnicity and Gender-ethnicity Subgroups

Using JNC VII Cut-points for Blood Pressure

52 Correlations between Selected Socio-demographic Variables and the Behavioral/Environmental Risk Factors of the Participants by Gender

53 Correlations between Selected Socio-demographic Variables and the behavioral/Environmental Risk Factors of the Participants by Ethnicity

54 Correlations between Selected Socio-demographic Variables and the Physiological Risk Factors of the Participants by Gender

55 Correlations between Selected Socio-demographic Variables and the Physiological Risk Factors of the Participants by Ethnicity

56 Correlations between Selected Dietary Components and Physiological Risk Factors of the Participants by Gender

57 Correlations between the Behavioral/Environmental and Physiological Risk Factors of the Participants by Gender

58 Correlations between Selected Dietary Components and Physiological Risk Factors of the Participants by Ethnicity

59 Correlations between Behaviora1/Environmental and

Physiological Risk Factors of the Participants by Ethnicity

60 Correlations between selected Socio-demographic Variables and the Emerging Risk Factors of the Participants by Gender and Ethnicity

61 Correlations between Selected Dietary Components and the Emerging Risk Factors of the Participants by Gender and Ethnicity

62 Correlations between the Behavioral/Environmental and the Emerging Risk Factors of the Participants by Gender and Ethnicity

63 Correlations between the Physiological and the Emerging Risk Factors of the Participant by Gender

64 Correlations between the Physiological and the Emerging Risk Factors of the Participant by Ethnicity 


\section{LIST OF FIGURES}

FIGURE

PAGE

1 Percentage Breakdown of Deaths from Cardiovascular Diseases: United States, 2001

2 Relative Risk of Future Cardiovascular Events

3 Prospective Studies of hs-CRP as a Marker for Future Cardiovascular Events in Populations of Apparently Healthy Men and Women (Without Known CHD)

4 Event-free Survival Among Women with hs-CRP and LDL-C Levels Above or Below the Median for the Study Population

5 Multivariable-adjusted Relative Risk of Cardiovascular Event by Framingham Risk Score

6 Cardiovascular Event-free Survival Among Apparently Healthy Individuals According to Baseline hs-CRP Levels

7 Distribution of the Participants by Countries of Birth

8 Distribution of the Participants' Parents by Countries of Birth 


\section{CHAPTER I}

\section{INTRODUCTION}

Cardiovascular disease (CVD) is a serious public health concern affecting more than 64 million Americans in 2001 (American Heart Association [AHA] Statistical Update, 2004). Since more than $55 \%$ of all CVD deaths are due to coronary heart disease (CHD), modification of the risk factors for CHD is important in the prevention of CHD (AHA Statistical Update, 2004). Evidence shows a decreasing trend in mortality due to CVD over the past 20 years (Braunwald, 1997; AHA Statistical Update, 2004; Rifai and Ridker, 2002). However, despite behavior/lifestyle changes and the use of new pharmacological drug therapies to decrease blood cholesterol (Shepard et al., 1995) and blood pressure levels (Kostis et al., 1997) CVD remains the leading cause of death in the United States (US) (Braunwald, 1997). Cardiovascular disease accounted for nearly 1 million deaths and over 6 million hospital discharges in 2001 and exacted a heavy burden on Black males and females (AHA Statistical Update, 2004). The incidence and prevalence of CVD will continue to increase as the population ages and, together may become one of the leading causes of disability-adjusted life years (DALYs) lost among working adults (Beaglehole et al., 2001; AHA Statistical Update, 2002). Nationally, the annual estimated direct and indirect cost of CVD in 2004 was over $\$ 368$ billion (AHA Statistical Update, 2004).

The current approach to the prevention of CHD is predicated on identification of risk factors and the quantitative assessment of short-term cardiovascular risk (Linton and Fazio, 2003). The Multiple Risk Factor Intervention Trial (MRFIT) (Stamler et al., 1986) 
and the Nurses' Health Study (Stampfer et al., 2000) have shown that the major risk factors for CHD (advancing age, cigarette smoking, elevated blood pressure [BP], total cholesterol [TC], low-density lipoprotein cholesterol [LDL-C], low levels of high-density lipoprotein cholesterol [HDL-C]) account for more than $70 \%$ of the excess risk for premature CHD. High fat diets, obesity and physical inactivity are now also considered major risk factors for CHD (National Cholesterol Education Program [NCEP]: Adult Treatment Panel [ATP] III, 2001). The measurement of serum lipids, especially LDL-C has been predictive of CHD (AHA, 2002; NCEP: ATP III, 2001).

Research indicates that $50 \%$ of males and $63 \%$ of females who died suddenly of CHD and had no previous symptoms of this disease (AHA Statistical Update, 2003), had normal or only slightly elevated lipid levels but, elevated high-sensitivity C-reactive protein (hs-CRP) levels, a marker of low-grade inflammation (Yu and Rifai, 2000; Rifai and Ridker, 2001). This is an indication that atherothrombosis can occur in the absence of hyperlipidemia (Ross, 1999; Ridker et al., 2002). Thus, in addition to the measurement of lipid parameters, measurements of hs-CRP have been shown to significantly improve the clinical prediction value in apparently healthy males and females, of first myocardial infarction (Ridker et al., 1998c; Ridker et al., 2000). This is important since apparently healthy young adults with normal lipid levels may have elevated hs-CRP levels and may be at risk for CHD.

\section{Statement of the problem}

The non-Hispanic Black (interchangeable with African American) population, now the second largest ethnic group in the US (US Census Bureau, 2000), is a composite 
of blacks born inside and outside the US, including groups such as foreign- and US-born Afro Caribbean Americans, a growing population in South Florida. In the year 2000, there were over 2.8 million foreign-born Caribbean Nationals in the US (US Census Bureau: Current Population Reports [P23-206], 2001). In March 2002, this population increased to over 3.1 million, with 55\% living in South Florida (US Census Bureau: Current Population Report [P20-539], 2003; US Census Bureau: Current Population Survey [CENBR], 2002). As this population increases it is important for healthcare professionals and health policy planners to monitor the health status of this and other foreign-born population. This is because their health care needs and access to quality health care may differ from that of US-born individuals (Stephen et al., 1994).

There is evidence showing lower all-cause mortality due to CHD in foreign-born Blacks compared to US-born Blacks and US-born Whites (Fang et al., 1997; Singh and Siahpush, 2002). In these studies, blacks included both non-Hispanic Blacks and Hispanic Blacks. Fang et al (1996) also showed that Caribbean Americans in New York City had substantially lower all-cause and cardiovascular mortality rates compared to Blacks born in the southern and northeastern US. However, risk factors for CHD were not investigated in this study.

Most of the studies investigating risk factors for CHD (including hs-CRP levels and TC/HDL-C ratio) have been done with adults aged between 40 years and older. There is a dearth of information on socio-demographic (educational attainment, household income, perceived stress, knowledge of risk factors for CHD, family history of CHD, medical insurance coverage, visitations to healthcare professionals), behavioral/environmental (atherogenic diet, physical inactivity, elevated body mass 
index, cigarette smoking and alcohol consumption,), physiological (elevated blood pressures, blood glucose, TC, LDL-C, TG and low HDL-C) and emerging (elevated hs$\mathrm{CRP}, \mathrm{TC} / \mathrm{HDL}-\mathrm{C}$ ratio) risk factors for CHD in foreign-born and US-born Caribbean Americans, especially younger adults (18-40 years).

Although national health data on African-Americans have been collected since the 1980 s, the data is of a composite nature and may have included foreign-born Afro Caribbeans. Also, it is being suggested that studies which compare minority populations with the majority 'White' population may give the impression that the latter group represented some kind of a health "norm" and that ethnic differences may represent some sort of a 'deviant' health state (Curtis and Lawson, 2000). Thus, it is suggested that we interpret these comparative studies with care.

\section{Significance}

Despite guidelines for the management of individual risk factors for CHD (National High Blood Pressure Education Program [JNC, VI, 1997], American Diabetes Association, 2002, NCEP: ATP III, 2001), CHD remains the number one killer of Americans (Braunwald, 1997; Beaglehole et al., 2001), thus the search for other (emerging) risk factors for CHD. Early detection of risk factors is important in reducing the severity and age of onset of CHD. The Healthy People 2010 Heart disease and Stroke Goal is to improve cardiovascular health and quality of life through the prevention, detection and treatment of risk factors; early identification and treatment of heart attacks and strokes; and prevention of recurrent cardiovascular events (HP 2010). The prevalence of risk factors for CHD is higher in non-Hispanic Blacks and other ethnic groups 
compared to non-Hispanic Whites (Gillum, 1988) and, in the absence of these risk factors, death from CHD is rare (Beaglehole et al., 2001).

Ethnic populations' beliefs and practices can influence the interactions between healthcare provider and individuals of this population. However, insufficient attention is paid to the area of ethno-medicine (Bronner, 1994). Information about an individual ethnic group is important for the identification, tracking and investigation of the reasons for ethnic differences in the prevalence and severity of disease and in the response of diseases to treatment (Burchard et al., 2003). Also, information about a person's ethnic group is important in identifying different risk factor profiles even when a disease does not occur with dramatically different frequencies in the different ethnic groups (Burchard et al., 2003). It is suggested that by focusing on the ethnic and cultural differences in a population we can better understand the variations within and between different ethnic and cultural groups and, the influence of birth origin on the prevalence of risk factors for and, severity of diseases (Burchard et al., 2003). This focus can provide the opportunity for healthcare professionals to develop culturally sensitive programs and strategies for the improvement of health outcomes.

No study has been done to evaluate the socio-demographic, behavioral/environmental, physiological and emerging risk factors of CHD in foreignborn and US-born Caribbean Americans, especially in South Florida, where there is a growing population. Foreign-born Afro Caribbeans may have different health perceptions, beliefs, behaviors, dietary habits and propensities to report health/nutritional conditions compared to US-born Caribbean Americans and African Americans. Also, there may be differences in socio-economic status, perception of stress, exposure to 
knowledge about CHD, eating habits, physical activity, obesity status, cigarette smoking and alcohol consumption. These differences may be distinguishing features of these groups and may influence risk factors for cardiovascular disease in this population of African ancestry (Curtis and Lawson, 2000). Designing effective prevention programs to reduce the risk of CHD requires responding to the needs of different ethnic groups (Dundas et al., 2001).

This research determined and compared socio-demographic (educational attainment, household income, perceived stress, knowledge of risk factors for CHD, family history of CHD, medical insurance coverage, visitations to healthcare professionals), behavioral/environmental (atherogenic diet, physical inactivity, elevated body mass index, cigarette smoking and alcohol consumption), physiological (elevated BP, fasting BG, serum TC, LDL-C, triglyceride (TG), low HDL-C) and emerging (elevated hs-CRP and TC/HDL-C ratio) risk factors for CHD in apparently healthy foreign-born Afro Caribbean (FBCA), US-born Afro Caribbean American (USBCA) and African American (AA) adults (18-40 years old). Data from this study may prove useful to healthcare professionals managing CHD risk factors reduction among this heterogeneous population.

\section{Research Questions}

1. Are the socio-demographic profiles of FBCA, USBCA and AA adults similar?

2. Are FBCA adults less likely to have behavioral risk/environmental risk factors of CHD as compared to USBCA and AA adults? 
3. Are FBCA adults less likely to have physiological risk factors of $\mathrm{CHD}$ as compared to USBCA and AA adults?

4. Are FBCA adults less likely to have emerging risk factors of $\mathrm{CHD}$ as compared to USBCA and AA adults?

\section{Purpose and Specific Objectives}

Ethnicity is a construct that encompasses both genetic and cultural differences (Anand, 1999; Yusuf et al., 2001). Thus, the purpose of the proposed research was: to determine and compare socio-demographic, behavioral/environmental, bio-physiological and emerging risk factors for CHD in apparently healthy FBCA, USBCA and AA adults (18-40 years old) in South Florida.

The following risk factors were included in the study:

1. Socio-demographic risk for $\mathrm{CHD}$ such as:

(a) Educational Attainment: was self-reported using the self-administered sociodemographic questionnaire (SDQ) as levels of educational attainment.

(b) Household income: was self-reported using the self-administered SDQ as levels of household income.

(c) Perceived Stress: was self-administered using the Cohen Perceived Stress scale (PSS). It contains 14 items and uses a five-point Likert scale (never, almost never, sometimes, fairly often, very often) to assess perceived stress levels.

(d) Knowledge of CHD risk factors: was self-administered using the "Knowledge of CHD Risk Factors" questionnaire. Subjects answered a set of questions that 
measured knowledge of CHD risks. The questionnaire contains 25 ' $\mathrm{T}$ ' or ' $\mathrm{F}$ ' responses.

(e) Family history of CHD: was self-reported using the self-administered SDQ as knowing or not knowing their family history of CHD.

(f) Medical insurance coverage: was self-reported using the self-administered SDQ as no coverage and type of insurance coverage.

(g) Visitations to healthcare professionals: was self-reported using the selfadministered SDQ as number of visits during the past year.

2. Behavioral and environmental risks for CHD such as:

(a) Dietary Intakes: was self-administered using the validated food frequency questionnaire (FFQ). Dietary intakes of carbohydrate, total fat, saturated fat, monounsaturated and polyunsaturated fats, fiber, fruits, vegetables and sodium were determined.

(b) Physical Activity: (PA) was self-administered using the Baecke questionnaire. This questionnaire assesses leisure time and occupational activities.

(c) Anthropometry: body mass index (weight (kilograms, kg) divided by height (meters, $\mathrm{m}^{2}$ ), was calculated using weight and height measurements of the subjects taken at the Nutrition Laboratory at Florida International University.

(d) Smoking status: was self-reported on the self-administered validated FFQ, as current smoker (number of cigarettes smoked per day) or not currently smoking.

(e) Alcohol Consumption: was self-reported on the self-administered validated FFQ as frequency of alcohol consumption per year/month/week/day. 
3. Bio-physiological risk factors for CHD such as:

(a) Blood pressure: (BP) was measured by a trained technician.

(b) Fasting blood glucose levels were measured by automated standard laboratory procedures.

(c) Total cholesterol (TC), high-density lipoprotein cholesterol (HDL-C), and triglyceride (TG) levels were measured by automated standard laboratory procedures. Serum LDL-C was calculated by the Friedwald equation.

4. Emerging markers of CHD risk such as:

(a) Fasting high-sensitivity C-reactive protein (hs-CRP) levels were measured by the Food and Drug Administration (FDA) approved latex-enhanced highsensitivity immunoephelometric assays on a BN II analyzer (Dade Behring, US).

(b) TC/HDL-C ratio was calculated by dividing TC concentration (mg/dL) by HDLC concentration (mg/dL).

Other cardiovascular diseases with significant public health impact include stroke, valvular heart disease, Chagas disease and heart failure (Yusuf et al., 2001). However, for the purpose of this study, the review of literature was limited to:

(a) Socio-demographic risk factors such as: socio-economic status (SES), perceived stress, knowledge of risk factors for CHD, family history of CHD,

(b) Behavioral/Environmental risk factors for $\mathrm{CHD}$ such as: atherogenic diet, physical inactivity, elevated body mass index (BMI), cigarette smoking and alcohol consumption, 
(c) Bio-physiological risk factors for CHD such as: elevated blood pressure, fasting blood glucose, TC, LDL-C, TG and low HDL-C levels and, (d)Emerging risk factors for CHD such as: elevated hs-CRP and TC/HDL-C ratio. 


\section{CHAPTER II}

\section{LITERATURE REVIEW}

\section{Cardiovascular Disease and Coronary Heart Disease}

Total cardiovascular disease (CVD) comprises all "diseases of the circulatory system" (International Classification of Diseases, $10^{\text {th }}$ revision [ICD/10] codes 100-199), and includes rheumatic fever/rheumatic heart disease (codes 100-109); hypertensive diseases (codes 110-115); ischemic (coronary) heart disease (codes 120-125); pulmonary heart disease and diseases of pulmonary circulation (codes 126-128); other forms of heart disease (codes 130-152); cerebrovascular disease (stroke) (codes 160-169); atherosclerosis (code 170); other diseases of the arteries, arterioles and capillaries (codes 171-179); diseases of the veins, lymphatic and lymph nodes, not classified elsewhere (codes 180-189); and other and unspecified disorders of the circulatory system (codes 195-199). Depending upon availability, data for congenital cardiovascular defects (codes Q20-Q28) are also included (American Heart Association [AHA] Statistical Update, 2003).

There is a decrease in mortality due to cardiovascular diseases (CVD) over the past 20 years. Indeed, during 1989-1999 the death rate from CVD declined by $15.6 \%$ (Braunwald, 1997; AHA Statistical Update, 2002; Rifai and Ridker, 2002) and continues to decline (AHA Statistical Update, 2004). However, despite lifestyle behavior changes and the use of new pharmacological drug therapies to decrease blood cholesterol (Shepard et al., 1995) and blood pressure levels (Kostis et al., 1997) CVD remains the leading cause of death among Americans of all race/ethnic groups and accounts for over 12 million deaths ( 7 million to coronary heart disease [CHD] and 5 million to stroke) 
annually, worldwide (Beaglehole et al, 2001). It is estimated that over 2,600 Americans die each day from CVD, an average of 1 death every 34 seconds (AHA Statistical Update, 2004). The incidence and prevalence of CVD will continue to increase as the population ages and, may become one of the leading causes of disability-adjusted life years (DALYs) lost among working adults (Beaglehole et al., 2001; AHA Statistical Update, 2002). Nationally, the annual estimated cost of CVD in 2004 is expected to be over $\$ 368.4$ billion, $\$ 226.7$ billion in direct costs and $\$ 141.7$ due to indirect costs (AHA Statistical Update, 2004).

In 2001, the age-adjusted prevalence of CVD in the US among non-Hispanic White males (30.0\%) and females (23.8\%) was lower compared to non-Hispanic Black males (40.5\%) and females (39.6\%). The age-adjusted prevalence for Mexican-American males $(28.8 \%)$ and females $(26.6 \%)$ was similar to the values seen in non-Hispanic Whites (AHA Statistical Update, 2004; NHANES III, 1988-94). Although more White males $(374,280)$ and females $(433,867)$ died from CVD compared to Black males $(48,939)$ and females $(56,821)$, the overall CVD mortality for 2001 was lower in White males $(384.3 / 100,000)$ and females $(273.6 / 100,000)$ compared to Black males $(510.5 / 100,000)$ and females $(376.6 / 100,000)$ (AHA Statistical Update, 2004; NHANES III, 1988-94). Also, higher numbers of CVD risk factors were seen among non-Hispanic Black and Mexican-American females than among non-Hispanic White females of comparable socio-economic status (NHANES III, 1988-94).

In the year 2000, Florida was ranked $19^{\text {th }}$ in age-adjusted CVD deaths with a death rate of $309.4 / 100,000$ population, a $16.3 \%$ decrease since 1990 , compared to the national average of $345.3 / 100,000$ population, a $16.5 \%$ decrease since 1990 (AHA 
Statistical Update, 2004). In Florida during the year 2000, there were more than 64, 000 deaths due to CVD (39,000 due to coronary heart disease and 10,000 due to stroke) and, 229,900 hospital discharges due to CHD and stroke (Florida Department of Health [FLDH], 2001). Cardiovascular disease is not just a disease of the elderly. In Florida during the year 2000, there were nearly 5,000 heart attack deaths and more than 1,100 stroke deaths in individuals under the age of 65 years. Also, there were more than 71,000 hospital discharges for heart disease and stroke among individuals under the age of 65 years (FLDH, 2001).

The major cardiovascular event is CHD and is defined as symptomatic ischemic heart disease including myocardial infarction (ICD/10 codes 121-122), stable or unstable angina (ICD/10 code 120 ), atherosclerotic cardiovascular disease (ICD/10 code 125.0), demonstrated myocardial ischemia by non-invasive testing and history of coronary artery procedures, and all other forms of chronic ischemic heart disease (ICD/10 codes 125.1125.9) (National Cholesterol Education Program [NCEP]: Adult Treatment Program [ATP] III, 2001; AHA Statistical Update, 2002). Coronary heart disease occurs when the coronary vessels (blood vessels that carry oxygen and nutrients to the heart) are clogged or blocked. If enough oxygen-carrying blood cannot reach the heart, the individual will feel pain in the chest or left arm or shoulder, called angina pectoris. If the blood supply is cut off completely, the individual will suffer a heart attack (AHA Statistical Update, 2002; Healthy People [HP], 2010). Coronary heart disease and stroke combined account for $72 \%$ of the total cardiovascular events in the US (Figure 1). 
Figure 1 - Percentage Breakdown of Deaths from Cardiovascular Diseases: United States, 2001.

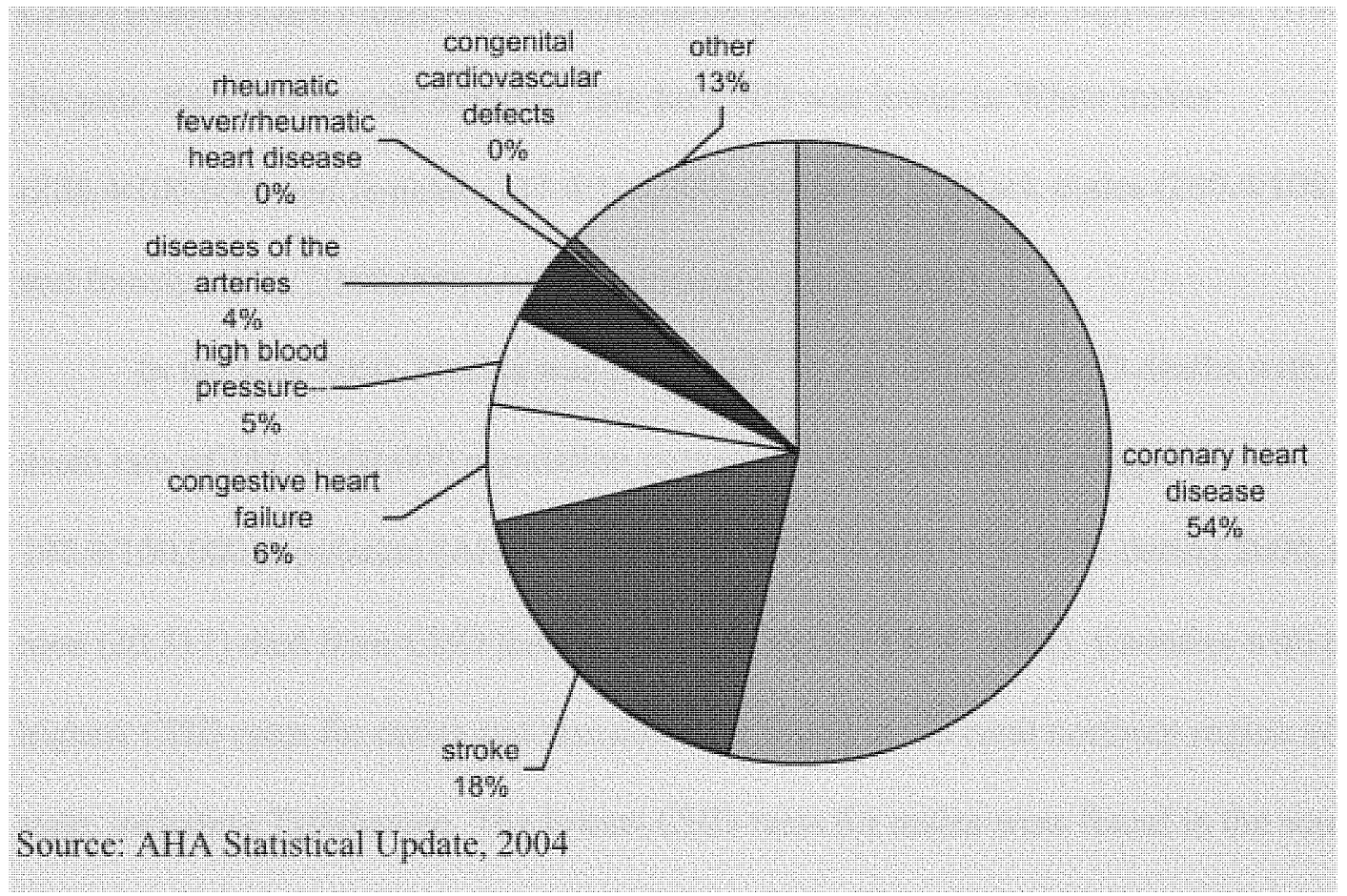

In the year 2001, over 13 million persons in the US (6.4\% of the population) had CHD whereas 4.8 million persons ( $2.0 \%$ of the population) had cerebrovascular disease, ischemic stroke being the major cerebrovascular event (AHA Statistical Update, 2004). Individuals with CHD often suffer from stroke and those with stroke often suffer from CHD (Assmann et al., 1999). In the US, a coronary event occurs every 26 seconds and every minute someone will die from one. Someone suffers a stroke every 53 seconds and every 3 minutes someone will die from one (AHA Statistical Update, 2004). Coronary heart disease is also the leading cause of premature, permanent disability in the US labor force, accounting for $19 \%$ of disability allowances paid by the Social Security Administration (AHA Statistical Update, 2002). In 1998, \$10.6 billion was paid to Medicare beneficiaries for CHD $(\$ 10,428$ per discharge for acute myocardial infarction; 
$\$ 11,399$ per discharge for coronary atherosclerosis and $\$ 3,617$ per discharge for other

CHD) (Health Care Financing Administration [HCFA] Statistical Supplement, 2000). In the year 2001, the overall mortality rate for CHD was lower in White males

$(228.4 / 100,000)$ and females $(137.4 / 100,000)$ compared to Black males $(260.0 / 100,000)$ and females $(176.7 / 100,000)$ (AHA Statistical Update, 2004).

In the year 2000, Florida was ranked $35^{\text {th }}$ in age-adjusted CHD deaths with a death rate of $191.3 / 100,000$ population compared to the national average of 188.9/100,000 population (AHA Statistical Update, 2004). In Florida during 2000, more men $(20,317)$ died from CHD than women (19,381) (FLDH, 2001). Counties with the highest $\mathrm{CHD}$ rates for males and females were found in the rural northern region of the state, including Tallahassee. The lowest CHD rates for males were found in the southern region of the state, including the cities of Miami, Fort Lauderdale and Fort Myers, whereas females in the city of Miami experienced intermediate rates of CHD deaths (Center of Disease Control [CDC] Fact sheets, 2002).

\section{Culture, Migration, Ethnicity and CHD}

The beliefs, traditions and practices by a community of individuals are classified as culture. These behavioral patterns are reinforced when the community is isolated by geography or segregated by socioeconomic status. This set of behavioral patterns is learned, not inherited, and is passed from one generation to the next through a process called enculturation (Plawecki, 1992). Cultural membership is defined by the term ethnicity (Brookins, 1993; Kittler and Sucher, 2001) which is a much broader construct that takes into account cultural traditions, common history and shared genetic heritage 
(Burchard et al., 2001). When individuals from one ethnic group migrate to an area with different cultural norms, these individuals will start to adapt to the cultural norms of the major society through a process known as acculturation. This occurs through a continuum of behavioral patterns (Kittler and Sucher, 2001). Some individuals become bicultural (practicing both cultures) whereas, others, especially second and third generation migrants may shed their ethnic identity and fully merge into the cultural norms of the majority culture, a process known as assimilation (Brookins, 1993; Kittler and Sucher, 2001). Although Bronner (1994) has suggested that individuals from a given culture may have similar health beliefs, attitudes, traditions and practices, the population may by no means be homogenous. Individuals may differ by socio-economic status, behavioral attitudes, exposure to stress, biophysical and biochemical parameters, country of origin and length of time in the US.

Individuals who migrate to other countries often carry patterns of disease and behavioral habits from their countries of origin to their countries of adoption. This has been shown in studies investigating risk for CHD in migrating Japanese (Robertson et al., 1977), Chinese (Gerber and Madhaven, 1980) and Filipinos (Gerber, 1980). However, as these individuals assimilate into the dominant society they may reflect the disease patterns of this society. This was seen in Southern-born Blacks who migrated to other regions in the US having higher mortality rates compared to Blacks native to their adopted region but below those of the south (Greenberg and Schneider, 1992). There are reports documenting this geographical transition of CHD risks. For example, in the NiHon-San study of Japanese migrants, low total cholesterol (TC) levels and CHD rates were seen in Japanese men in Japan whereas intermediate and high TC levels and CHD 
rates were seen in Honolulu and San Francisco, respectively (Kagan et al., 1974). Additionally, in investigating the rates of non-insulin dependent diabetes mellitus (NIDDM) among the population of the African Diaspora in Nigeria, Barbados, Jamaica, St. Lucia, the United States (US) and the United Kingdom (UK), Cooper et al (1997) showed that the age-adjusted prevalence rates for NIDDM was $2 \%$ in Nigeria, $9 \%$ in the Caribbean and $11 \%$ in the US and UK, respectively. In investigating the distribution of blood pressures in seven populations of African origin (West Africa, Nigeria, Cameroon), the Caribbean (Barbados, Jamaica, St. Lucia), the US (metropolitan Chicago, Illinois), Cooper et al (1997a) observed a consistent gradient of hypertension prevalence from 16\% in West Africa to $26 \%$ in the Caribbean and $33 \%$ in the US. They suggested that environmental factors such as dietary intakes of sodium and potassium and obesity may be important determinants of this gradient.

Individuals living in the same environment have been evaluated with respect to morbidity and mortality differentials. Singh and Siahpush (2002) analyzed data from the 1979-1989 National Longitudinal Mortality Study (NLMS) (longitudinal data set for examining socioeconomic status, occupational and demographic factors associated with all-cause and cause-specific mortality in the US) and, the 1993-1994 National Health Interview Survey (household survey in which data on socioeconomic status, demographic, behavioral [smoking, alcohol use, diet, physical activity], morbidity, health care are collected). The NLMS sample consisted of 240,738 US-born non-Hispanic Whites; 25,655 US-born non-Hispanic Blacks; 2,189 US-born Asians and Pacific Islanders; 6,686 US-born Hispanics; 2,095 American Indians and, 13,073 foreign-born non-Hispanic Whites; 777 foreign-born Blacks; 3,520 foreign-born Asian and Pacific 
Islanders and 6,177 foreign-born Hispanics. They found that immigrants tended to have more favorable health-enhancing behavioral habits than their US-born counterparts. They also showed that Black migrants compared to their US-born counterparts were less likely to smoke cigarettes ( $10.4 \%$ vs. $29.3 \%$ ), less likely to be overweight $(25.2 \%$ vs. $38.4 \%)$, and less likely to be hypertensive ( $16.8 \%$ vs. $25.5 \%)$ but more likely to have no medical insurance (40.2\% vs. $34.5 \%$ ). In this study (Singh and Siahpush, 2002), Blacks consisted of a composite group comprising African Americans, Nigerian Americans, Black Puerto Ricans, Jamaican Americans, West Indian Americans and Haitian Americans.

A similar study by Fang et al. (1997) showed 46\%-51\% lower mortality among Black migrants compared to US-born counterparts in New York City. In this study, Blacks were also a composite of African Americans, Black Puerto Ricans, Jamaicans, Nigerians, West Indians and Haitians. In an earlier study, Fang et al. (1996) showed that Caribbean-born Blacks in New York City had substantially lower all-cause and cardiovascular mortality rates compared to Blacks born in the southern and northern region of the US. In this study Fang et al (1996) linked mortality data from 1988 through 1992 with 1990 US Census data for New York City. Mortality data for African Americans born in the southern US (Alabama, Arkansas, Delaware, Florida, Georgia, Kentucky, Louisiana, Maryland, Mississippi, North Carolina, Oklahoma, South Carolina, Tennessee, Texas, Virginia, West Virginia, District of Columbia), Northeastern US (Connecticut, Maine, Massachusetts, New Hampshire, New Jersey, New York, Pennsylvania, Rhode Island, Vermont) and the Caribbean (mostly from Barbados [86\%], Grenada, Haiti, Jamaica, Trinidad and Tobago) were compared with those for nonHispanic Whites born in the Northeastern US. They found that the age-adjusted death rate 
from CHD in Caribbean Americans 25 to 44 years of age was lower (7.4/100,000 population) compared to non-Hispanic Whites born in the Northeast $(16.8 / 100,000$ population) and, African Americans from the Northeast (23.1/100,000 population) and South (30.1/100,000 population). Also, higher educational and employment levels were seen in Whites compared to African-Americans. More Caribbean Americans compared to African Americans born in the South were high school graduates and their average length of stay in the US was 13.9 years. This study did not evaluate the risk factors for CHD and stroke.

A study from the National Academy of Sciences (Hernandez, 1999) has reported on the protective effect of immigrant status. This report demonstrated that foreign-born young adults experienced more favorable physical and emotional health and less involvement in risky behaviors than US-born young adults of foreign-born parents and US-born young adults of US-born parents. The report also stated that the protective quality of immigrant status was not related to the family or neighborhood context of immigrant children. Thus, it is suggested that future research should concentrate on uncovering the mechanism(s) behind the health protective effect(s) of foreign birth.

\section{Race/Ethnic Disparities in Cardiovascular Health}

Ethnicity is an important risk factor for CHD. In general, members of minority groups are at higher risk of $\mathrm{CHD}$, have greater prevalence of risk factors for $\mathrm{CHD}$ and have greater death rates from CHD compared to non-Hispanic Whites (FLDH, 2001). African Americans have the highest overall mortality from CHD of any ethnic group in the US, particularly at younger ages (Clark et al., 2001; Gillum, 1997; NCEP: ATP III, 
2001). In 1995, the age-adjusted death rate for heart disease was $42 \%$ higher in African American males than in White males and 65\% higher in African American females than in White females and almost twice as high in males than females (HP, 2010). Disparities also exist in treatment outcomes for individuals who have heart attacks. Females ( $44 \%$ of those who experienced heart attacks) have poorer outcomes following a heart attack compared to males (27\%) who experienced heart attacks (Gillum et al., 1997). These differences can be explained, partially by the presence of high blood pressure, diabetes, elevated serum lipids and, possibly congestive heart failure (Zoratti, 1998; AHA Statistical Update, 2002). Other factors such as age, gender, low socio-economic status, and prior medical conditions have been associated with longer pre-hospital delays in seeking medical care for symptoms of a heart attack (AHA statistical Update, 2002).

It is important to monitor the health status of ethnic groups. The US population profile is changing and, by the year 2010, the percentages of African Americans, Hispanics and other minority groups are expected to increase (HP 2010). However, race/ethnic gaps in health care and inadequate access to health care assistance will remain if appropriate intervention programs are not instituted (HP, 2010).

\section{African Americans, Foreign-born and US-born Afro Caribbean Americans}

The non-Hispanic Black population of over 35 million persons is the nation's second largest ethnic group, comprising $12.3 \%$ of the entire United States (US) population (US Census, 2000). This population is heterogeneous and includes several groups with different cultural backgrounds such as African Americans, Afro Caribbean Americans (US-born Afro Caribbeans), Afro Caribbean migrants and other foreign-born 
individuals of African ancestry (US Census, 2000). The estimated foreign-born

population of the US, in March 2000, was 28.4 million with over 2.8 million foreign-born Caribbean Nationals (US Census Bureau: Current Population Reports [P23-206], 2001). In March 2002, the population of foreign-born Caribbean Nationals was estimated to be over 3.1 million with 55\% living in South Florida (US Census Bureau: Current Population Report [P20-539], 2003).

Caribbean migrants are a diverse group of individuals from the Caribbean region, which stretches from Florida to Venezuela. This region includes independent states such as Antigua/Barbuda, the Bahamas, Barbados, Belize, Cuba, Dominica, Dominican Republic, Grenada, Guyana, Jamaica, Montserrat, St. Christopher/Nevis, St. Lucia, St. Vincent/Grenadines, Trinidad \& Tobago, Haiti and the US territory of Puerto Rico. Some of the islands, such as the Cayman Islands (Britain), Turks and Caicos Islands (US), the Virgin Islands (US) and, Martinique (France) are still under foreign control. Most of these islands are south of the State of Florida, between longitudes $85 \mathrm{~W}$ and $59 \mathrm{~W}$ and latitudes $10 \mathrm{~N}$ and $25 \mathrm{~N}$. Belize and Guyana are on the mainland of Central and South America, respectively. Jamaican and Haitians are the two major groups contributing to the Afro Caribbean population in the US. Jamaicans are concentrated in Fort Lauderdale (46\%), New York (40\%), Nassau-Suffolk (39\%), Washington DC (49\%) and Atlanta (53\%), whereas Haitians are more populous in Miami (61\%), West Palm Beach (62\%), Boston (57\%) and Newark (50\%) (Logan and Deane, 2003).

Of the estimated 3.1 million foreign-born Caribbean Nationals living in the US, over 1.5 million are classified as Afro Caribbean and, since 1990, the Afro Caribbean 
population has increased by over $60 \%$ from 924,697 in 1990 to $1,542,895$ in 2000 with $60 \%$ being foreign-born (Table 1) (US Census, 2000; Logan and Deane, 2003).

Table 1 - Composition and Growth of the non-Hispanic Black Populations of the US, $1990-2000$

\begin{tabular}{|c|c|c|c|c|c|c|c|}
\hline & \multicolumn{2}{|c|}{ Population (000) } & \multicolumn{2}{|c|}{$\begin{array}{l}\text { Percent of Black } \\
\text { Population (\%) }\end{array}$} & \multicolumn{2}{|c|}{$\begin{array}{l}\text { Percent of Total } \\
\text { Population }(\%)\end{array}$} & $\begin{array}{l}\text { Growth } \\
(\%)\end{array}$ \\
\hline & 1990 & 2000 & 1990 & 2000 & 1990 & 2000 & $1990 / 2000$ \\
\hline AA & 28,034 & 33,048 & 96.0 & 93.9 & 11.3 & 11.7 & 17.9 \\
\hline $\mathrm{AC}$ & 924 & 1,542 & 3.2 & 4.4 & 0.4 & 0.5 & 66.9 \\
\hline African & 229 & 612 & 0.8 & 1.7 & 0.1 & 0.2 & 166.9 \\
\hline NHB & 29,188 & 35,203 & & & 11.7 & 12.5 & 20.6 \\
\hline Total US & 248,709 & 281,421 & & & 100.0 & 100.0 & 13.2 \\
\hline
\end{tabular}

Source: US Census, 2000; Logan and Deane, 2003 (Adapted)

$\mathrm{AA}=$ African Americans

$\mathrm{AC}=$ Afro Caribbean

NHB $=$ Non-Hispanic Black

In Florida, Miami has the highest proportion of foreign-born individuals. This population will increase in the future as migrants form families. One indication of this is the increase in the proportion of births to foreign-born women residing in the US from $6 \%$ in 1970 to over $20 \%$ in 1999 (US Census Bureau: Current Population Survey [CENBR], 2002). Individuals from the Caribbean Region are the fastest growing ancestry group in Broward, Palm Beach and Miami-Dade Counties in South Florida (US Census, 2000). For the year 1990 to 2000 , the Caribbean population grew by $173 \%$ (from 55,197 to 150,476 ) in Broward County, $142 \%$ (from 20,441 to 49,402) in Palm Beach County and $45 \%$ (from 105,477 to 153,255 ) in Miami-Date County (US Census, 2000). In spite of this increase in the foreign-born population of the US, there are relatively few studies 
investigating differentials in nutrition/health and mortality patterns between foreign-born and US-born individuals (Singh and Siahpush, 2002), especially Caribbean Americans.

In 2000, the average African American had a lower median household income $(\$ 35,679$ versus $\$ 41,321)$ and educational attainment $(17.5 \%$ college education versus 20.3\% college education) compared to Afro Caribbeans. Also, more African Americans were unemployed (11.2\% versus $8.7 \%)$ and below the poverty threshold ( $30.4 \%$ versus $18.8 \%$ ) compared to Afro Caribbeans. However, more African Americans owned their homes (53.1\% versus $49.8 \%$ ) compared to Afro Caribbeans (Table 2) (Logan and Deane, 2003).

Table 2 - Socio-economic Characteristics of the Non-Hispanic Black Populations in Comparison with Other US Racial and Ethnic Groups, 1990 - 2000

\begin{tabular}{|l|l|l|l|l|l|l|}
\hline & $\begin{array}{l}\text { Population } \\
(000)\end{array}$ & $\begin{array}{l}\text { Foreign- } \\
\text { born (\%) }\end{array}$ & $\begin{array}{l}\text { Years of } \\
\text { Education }\end{array}$ & $\begin{array}{l}\text { Median } \\
\text { Household } \\
\text { Income }\end{array}$ & $\begin{array}{l}\text { Unemploye } \\
\mathrm{d}(\%)\end{array}$ & $\begin{array}{l}\text { Below } \\
\text { Poverty } \\
(\%)\end{array}$ \\
\hline $\mathbf{1 9 9 0}$ & & & & & & \\
AA & 28,034 & 1.8 & 11.7 & $\$ 29,251$ & 12.5 & 32.8 \\
AC & 924 & 72.4 & 12.1 & $\$ 42,927$ & 9.4 & 17.8 \\
African & 229 & 72.1 & 14.3 & $\$ 35,041$ & 8.5 & 24.7 \\
NHB & 29,188 & 4.7 & 11.7 & $\$ 29,850$ & 12.3 & 32.3 \\
& & & & & & \\
$\mathbf{2 0 0 0}$ & & & 12.4 & $\$ 33,790$ & 11.2 & 30.4 \\
AA & 33,048 & 2.2 & 12.6 & $\$ 43,650$ & 8.7 & 18.8 \\
AC & 1,542 & 68.3 & 14.0 & $\$ 42,900$ & 7.3 & 22.1 \\
African & 612 & 78.5 & 12.5 & $\$ 34,300$ & 11.0 & 29.7 \\
NHB & 35,203 & 6.4 & & & & \\
\hline
\end{tabular}

Source: Logan and Deane, 2003 (Adapted)

$\mathrm{AA}=$ African Americans

$\mathrm{AC}=$ Afro Caribbean

$\mathrm{NHB}=$ Non-Hispanic Black 
Genetics, cultural patterns and behavioral/environmental factors may influence differences in morbidity and mortality from diet-related diseases in this population (Kumanyika, 1993; Department of Health and Human Services [DHHS], 1991). Also, subgroups within this Black population may have different cultural and lifestyle habits that may influence the direction of risks and risk factors for CHD.

During the past two decades, changes in dietary, nutrition and health behaviors (Popkin, 1994) have occurred in the Caribbean, reflective of the close ties with the US and the introduction of American dietary and behavioral habits in the region. The consequences of these transitions have been an increase in chronic degenerative conditions, such as cardiovascular disease, diabetes mellitus and obesity, now the main causes of death (Sinha, 1995; Jackson, 2001; Henry, 2001).

Most of the studies investigating risk factors for CHD (including CRP levels and $\mathrm{TC} / \mathrm{HDL}-\mathrm{C}$ ratio) have been done with adults aged between 40 and 84 years. There is a dearth of information on CHD risk factors (such as obesity, cigarette smoking, blood pressure values, blood glucose and lipids levels) in foreign-born and US-born Caribbean Americans. Also, the influence of diet, physical activity, perceived stress and knowledge of $\mathrm{CHD}$ on traditional risk factors for CHD is unknown in this population. In addition, there is no information on CRP levels and TC/HDL-C ratio in this population.

Nutritional and health information on African Americans may not be applicable to Caribbean migrants and Caribbean Americans. Caribbean migrants may have different health perceptions, beliefs, behaviors, dietary habits and propensities to influence nutritional/health conditions compared to African Americans and possibly Caribbean Americans. Also, there may be differences in exposure to knowledge about CHD, 
physical activity and stress. These differences may be distinguishing features among the groups and, may influence risk factors for cardiovascular disease between the population groups (Curtis and Lawson, 2000).

Thus, the Healthy People 2010 Heart Disease and Stroke (HP 2010: Priority 12) goal is to improve cardiovascular health and quality of life through the prevention, detection, and treatment of risk factors; early identification and treatment of heart attacks and strokes, and prevention of recurrent cardiovascular events in the US (HP 2010).

\section{Risk Factors for CHD}

Risk factors are characteristics, attributes or behaviors that increase an individual's risk or chances of developing or dying from a disease. There are several well-known modifiable and non-modifiable risk factors for CHD. Modifiable risk factors are those attributes or behaviors that increase our risk of developing or dying from CHD. These include an atherogenic diet (poor nutrition), physical inactivity, overweight and obesity, stress, tobacco use, elevated blood pressure, blood glucose and lipids. Each of these behaviors or conditions can be changed with appropriate intervention (AHA Statistical Update, 2002; NCEP: ATP III, 2001). In the absence of these risk factors death from CHD is non-existent (Beaglehole et al., 2001). Non-modifiable risk factors for CHD are those that cannot be changed and include advancing age, gender (males are at higher risk of CHD compared to females), and family history of CHD (AHA Statistical Update, 2002; NCEP: ATP III, 2001). Additionally, the population attributable risk percent (PAR\%) represents the percentage of all CHD deaths that could be avoided if a particular risk factor was eliminated from the population. For example, in Florida during 2001, 
elevated cholesterol and physical inactivity were responsible for an estimated $43 \%$ and $35 \%$ of all CHD deaths, respectively (Table 3) (FLDH, 2001).

Table 3 - Population Attributable Risk Percent for CHD and Stroke

\begin{tabular}{|c|c|c|}
\hline Population Attributable Risk & CHD & Stroke \\
\hline Physical inactivity & $35 \%$ & $*$ \\
\hline Obesity & $17 \%$ & $20 \%$ \\
\hline Cigarette use & $22 \%$ & $12 \%$ \\
\hline High blood pressure & $25 \%$ & $26 \%$ \\
\hline Diabetes & $8 \%$ & $3 \%$ \\
\hline Elevated cholesterol & $43 \%$ & $10 \%$ \\
\hline
\end{tabular}

*Data not available

Source: FLDH, 2001

\section{Socio-demographic Variables}

\section{Age}

As individuals age there is a progressive accumulation of coronary plaque due to cumulative exposure to $\mathrm{CHD}$ risk factors, both known and unknown. The plaque can rupture, causing acute coronary events such as unstable angina or myocardial infarction, and/or increase in size causing coronary obstructive symptoms, such as angina pectoris (NCEP: ATP III, 2001).

\section{Gender}

At any given age males have a greater risk for CHD and stroke than females (Wilson et al., 1998; NCEP: ATP III, 2001; AHA Statistical Update, 2002). The incidence of CHD in women lags behind men by 10 years for total CHD and by 20 years for more serious clinical events such as myocardial infarction and sudden death. The 
reasons for a gender difference in risk for CHD are not fully understood. It is suggested that the amount of sex hormones in the blood may influence the risk of CHD and stroke (AHA Statistical Update, 2002). There is evidence suggesting that men suffer more CHD events than women before women reach the age of menopause. If menopause is caused by surgery to remove the uterus and ovaries, the risk of CHD rises sharply, however, if menopause occurs naturally, the risk rises more slowly (AHA Statistical Update, 2002).

\section{Socio-economic Status (SES) and Stress}

Individuals with lower income and educational levels have a higher risk for stroke and CHD (AHA Statistical Update, 2002; Kaplan and Keil, 1993). African American and non-Hispanic White males with lower socio-economic status (SES) have been shown to have a higher risk of death from CHD than non-Hispanic White males of higher SES (Tyroler et al., 1984). Inversely, Keil et al (1984) showed that African American males with high SES had half the CHD of other African-American males and, experienced no angina pectoris, acute myocardial infarction (AMI) death or sudden death during the observation period of the study. Obviously, there are marked socio-economic differences in CHD morbidity and mortality (Wood et al., 1998). This may be partly explained by socio-economic differences in CHD risk factors such as smoking, blood pressure levels, blood lipid and glucose levels (Jacobson and Thelle, 1988; Luepker et al., 1993). Another suggestion is that individuals from lower socio-economic groups may have poorer health habits and health knowledge (Wood et al., 1998). Other researchers have shown that SES may be associated with higher cumulative levels of stress and that biological reaction to 
this stress may contribute to hypertension (Kington and Smith, 1997; Williams, 1992), overeating and/or increase in cigarette smoking (AHA Statistical Update, 2002).

\section{Knowledge of Risk Factors for CHD}

Little is known about the attitudes and knowledge of CHD risk factors in individuals and the factors will motivate individuals to effect changes in their behaviors in an attempt to reduce the onset of chronic diseases, such as CHD (Shepherd, 1998; Erhardt and Hobbs, 2002). The Heart European Leaders Panel (HELP) study in 5 European countries (France, Germany, Italy, Sweden, United Kingdom) showed that a considerable degree of indifference to CHD existed, despite the possession of a reasonable level of knowledge of CHD risk factors, even among individuals with $\mathrm{CHD}$ (Shepherd, 1998). In a survey (Hyman et al., 1993) conducted to examine cholesterolrelated knowledge, attitudes and behaviors among 547 randomly selected individuals 18 years and older, $76 \%$ reported knowledge of high blood or serum cholesterol whereas $30 \%$ had never had a cholesterol screening. The individuals consisted of Hispanics (54\%), African-Americans (28\%), non-Hispanic Whites (14\%), Asians and Native Americans (4\%). From this same study, $30 \%$ of Hispanics reported that they never read food labels, which was approximately double the percentage of African-Americans and non-Hispanic White individuals who did not. In a study conducted by Levy et al (1993) consumer knowledge about dietary fat and cholesterol was poor. This suggested a relatively low level of awareness of risk factors for CHD. In a study by Gates and McDonald (1997) ethnic differences were found in knowledge, attitudes and nutrient intakes related to CVD. In this same study, compared to non-Hispanic White females, 
African American females consumed poorer diets, were less aware of the relationship

between nutrient intakes and health and had less knowledge about food sources of fat and cholesterol. However, these African-American females were more aware of the need to change their diets and were more likely to report that nutrition was important when they shop for food than the non-Hispanic White females. Compared to non-Hispanic Whites, African Americans and other ethnic groups disproportionately have higher prevalence of poverty, lack of education and lack of access to health care thus, exposing them to greater risk of chronic diseases (Gates and McDonald, 1997). There is no available data comparing knowledge of CHD risk factors differentials between foreign-born and USborn Afro Caribbean Americans.

\section{Family History of Premature CHD}

There is evidence to indicate that a family history of premature CHD (definite myocardial infarction or sudden death before 55 years of age in father or other male firstdegree relative, or before 65 years of age in mother or other female first-degree relative, is an independent risk factor for coronary events even when other risk factors are absent (Benson et al., 1999; Li et al., 2000; Williams et al., 2001). The relative risk for CHD in first-degree relatives has been reported to range from 2 to as high as 12 times that of the general population (NCEP: ATP III, 2001; Phillips et al., 1974; Rissanen, 1979) and, that risk increases with the number of primary relatives affected and at younger ages of onset of the coronary event (Pohjola-Sintonen et al., 1998; Rissanen and Nikkila, 1977). Children of parents with heart disease are more likely to develop the condition 
themselves, probably due to shared socio-cultural environment, exposures to similar behavioral habits and genetics (NCEP: ATP III, 2001).

Many prospective cohort and case-control studies, including the recent Atherosclerosis Risk in Communities Study (ARIC) in four US communities, have shown family history of CHD to be independent of the traditional risk factors (Benson et al., 1999; Sharrett et al., 1999). Many risk factors for CHD are under genetic control (such as blood pressure, serum lipids and obesity), but they account for only a portion of the aggregation of CHD seen in families (Snowden et al., 1982; Khaw and Barrett-Connor, 1986). A positive family history of CHD captures the underlying complexities of genegene and gene-environment interactions by identifying families with combinations of risk factors, both measured and unmeasured, that lead to disease expression. Also, a positive family history of CHD identifies the relatively small subset of families in the population at highest risk for CVD who may benefit most from targeted screening and intensive intervention (Hunt et al., 2003). However, the use of family history of CHD in risk evaluation has created some debate (Gotto et al., 2000). For example, in the US, guidelines (NCEP: ATP III, 2001) consider family history of CHD to be an important non-modifiable risk factor for CHD, whereas in Europe, calculations do not generally adjust for family history of CHD but only note that a positive family history for CHD increases the predicted risk (Gotto et al., 2000). 


\section{Behavioral/Environmental Risk Factors for CHD}

\section{Atherogenic Diet}

Poor nutrition and physical inactivity together are the second leading preventable cause of death in the US. Poor nutrition and physical inactivity are responsible for an estimated 300,000 deaths each year and are causally related to a variety of chronic diseases and conditions, including overweight and obesity, high blood pressure, hyperglycemia and diabetes, hypercholesterolemia, CHD, stroke, osteoarthritis and some cancers (FLDH, 2001). Dietary habits can modify and/or influence CHD and stroke risk factors in the population (United States Department of Agriculture [USDA], 2000; Krauss et al., 2000). For example, dietary intakes high in saturated fatty acids and cholesterol can influence LDL and HDL- cholesterol and obesity whereas high salt intakes can influence blood pressure in high-risk populations (NCEP: ATP III, 2001). Krauss et al. (2000) have also indicated that populations consuming diets high in fruits, vegetables, whole grains and unsaturated fatty acids may be at lower risk for many of the chronic degenerative conditions, such as CHD, obesity, hypertension and diabetes. The particular nutrients that influence this lower risk have not been adequately defined, however fiber (both soluble and insoluble) (Rimm et al., 1996a), antioxidant nutrients, such as carotenoids, flavanoids, Vitamins C and E, B-complex of vitamins, omega-3 fatty acids and other micronutrients have been implicated (Krauss et al., 2000). Also, components of antioxidant enzymes, such as selenium and copper can act singly or jointly to protect the arterial vessels from oxidative damage and, thus may influence CHD risk (Gey et al., 1993). 
Fatty Acids and Dietary Cholesterol: The American Heart Association recommends a diet low in saturated fat, trans fat, cholesterol and sodium and high in vegetables, fruits, whole grains, legumes, fat-free or low-fat dairy products, and dietary fiber (AHA Statistical Update, 2002). The total fat (saturated, trans, monounsaturated, polyunsaturated) intake should be less than $30 \%$ of total daily calories, saturated fat and trans fat should not exceed $10 \%$ of total daily calories for healthy individuals. There is evidence from animal experiments, observational studies, clinical trials and metabolic studies conducted in different populations showing a strong and consistent relationship between dietary fats and CVD, especially CHD (Kris-Etherton et al., 2001a). Saturated fatty acids raise total cholesterol (TC) and low-density lipoprotein cholesterol (LDL-C), however, individual fatty acids may have different effects (Katan et al., 1996; Mensink et al., 1992). Monounsaturated fatty acids (MUFA) and n-6 polyunsaturated fatty acids (PUFA) have been shown to reduce TC and LDL-C levels (Kris-Etherton, 1999). Hu et al. (1997), using 14-year follow-up data from the Nurses' Health Study, have shown that intakes of MUFA and PUFA were inversely associated with CHD risk, whereas saturated and trans fats were positively associated with CHD risk.

Between 1965 and 1991, total fat as a proportion of daily calorie intake and saturated fat intakes fell from $39.1 \%$ to $33.1 \%$ and $14.4 \%$ and $11.0 \%$, respectively among US adults 18 years and older. The average daily total fat intake in the US was 81.4 grams (96.5 $\mathrm{g}$ for males and 67.3 $\mathrm{g}$ for females). Non-Hispanic Whites had higher total fat intake ( $82.7 \mathrm{~g}$ ) (99.0 $\mathrm{g}$ for males and $67.4 \mathrm{~g}$ for females) compared to non-Hispanic Blacks (82.0 g) (94.6 $\mathrm{g}$ for males and 71.2 $\mathrm{g}$ for females) (NHANES III, [1988-94]). The

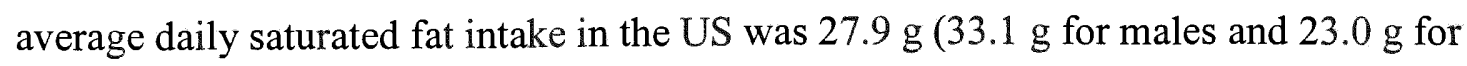


females). Non-Hispanic Whites also had higher saturated fat intake (28.4 g) (34.1 g for males and $23.1 \mathrm{~g}$ for females) compared to non-Hispanic Blacks (27.5 g) (31.7 $\mathrm{g}$ for males and $23.8 \mathrm{~g}$ for females).

Dietary cholesterol elevates plasma cholesterol levels (Hopkins, 1992), however, its association with CHD is still controversial (Hu et al., 1999). The recommended daily intake of dietary cholesterol for adults is less than 300 milligrams (mg) (NHANES III,[1988-1994]). The average daily intake of dietary cholesterol in the US was $269.6 \mathrm{mg}$ (323.5 mg for males and $218.9 \mathrm{mg}$ for females). Non-Hispanic Blacks consumed more dietary cholesterol (297.9 mg) (358.8 mg for males and $245.6 \mathrm{mg}$ for females) than nonHispanic Whites (259.3 mg) (312.6 mg for males and $209.1 \mathrm{mg}$ for females) (NHANES III, [1988-94]).

Dietary Fiber: Dietary fiber (soluble fiber-pectins, gums, mucilages, hemicelluloses; insoluble fiber-cellulose, hemicelluloses) has been shown to reduce TC and LDL-C (Anderson and Hanna, 1999). Several studies have demonstrated that a high fiber diet lowers the risk of CHD (Trushwell, 2002; Liu et al., 1999; Pietinen et al., 1996; Rimm et al., 1996). The recommended daily intake of fiber is $25 \mathrm{~g}$ or more while the average daily consumption of fiber in the US is $15.6 \mathrm{~g}(17.8 \mathrm{~g}$ for males and $13.6 \mathrm{~g}$ for females) (NHANES III,[1988-1994]). Non-Hispanic Blacks (13.4 g) (15.0 g for males and $12.0 \mathrm{~g}$ for females) consumed less fiber non-Hispanic Whites (15.8 $\mathrm{g}$ ) (18.1 $\mathrm{g}$ for males and 13.7 $\mathrm{g}$ for females) (NHANES III, [1988-94]). 


\section{African Americans and Afro Caribbeans Eating Habits}

The African American diet tends to be low in fruits and vegetables and high in fats and salt (Kant et al., 1991). Favorite food items of African Americans include fried chicken, barbecued ribs, corn bread, sweet potato pie, collard greens, and fruit-flavored drinks and juices (Kitler and Sucher, 2001). In addition, cookies or candies are preferred snack items (Kitler and Sucher, 2001). African Americans have adopted the traditional southern cuisine, known as "soul food", as an emblem of identity and recognition of Black history.

The traditional Caribbean diet is high in fiber and low in saturated fats and high in salt (Lemic-Stojcevic et al., 2001; Sharma et al., 1999). There are studies indicating that as individuals migrate from their region of origin to another country or region, they eventually practice the dietary habits of the dominant society (Sharma et al., 1999; Robertson et al., 1977; Gerber and Madhaven, 1980; Gerber, 1980). There is also evidence that as populations become more affluent they will also practice the dietary habits of more developed countries and experience increases in chronic degenerative conditions such as CHD, stroke, diabetes, obesity and some types of cancers (Sinha, 1995; Henry, 2001; WHO, 2000; Sharma et al., 1999). Sharma et al (1999) investigated nutrient intake trends among 102 male and 153 female Afro Caribbeans in Britain of which 18\% were born in England (second generation Afro-Caribbeans), 60\% in Jamaica, $6 \%$ in Barbados and $14 \%$ in other eastern Caribbean islands. They found that Caribbeanborn individuals (mean age 56 years, mean time in Britain 30 years) had significantly lower percent of energy from total fat (31.3\%) and saturated fat $(10.9 \%)$ than younger (mean age 29 years) British-born Afro Caribbeans (35\% and 12.6\%), respectively. The 
Caribbean-born individuals also ate more fruits (an additional $84 \mathrm{~g}$ per day) and vegetables (an additional $26 \mathrm{~g}$ per day) compared to British-born Afro Caribbeans. More than $30 \%$ of the adult population in Florida does not consume enough fruits and vegetables (FLDH, 2001). There is insufficient data on the dietary habits of Afro Caribbeans/Caribbean Americans living in the US.

\section{Physical Activity/Inactivity}

There are studies indicating that physical activity lowers LDL-C and TG levels, raises HDL-C cholesterol levels, improves insulin sensitivity and lowers blood pressure (Blair et al., 1983; King and Kriska, 1992; Helmrich et al., 1991; Haskell et al., 1994; NCEP: ATP III, 2001). Conversely, there is evidence indicating that physical inactivity is a major risk factor for CHD (NCEP: ATP III, 2001; Fletcher et al., 1994; US Surgeon General Report, 1996). The relative risk of CHD associated with physical inactivity ranges from 1.5 to 2.4 , an increase in risk comparable to that observed for high blood pressure, high blood cholesterol or cigarette smoking (AHA Statistical Update, 2004; Pate et al., 1995). The mechanisms whereby physical inactivity raises the risk for CHD are not fully understood and may be multi-factorial in nature (NCEP: ATP III, 2001). Individuals who are physically inactive reduce their caloric expenditure and this may contribute to obesity and its associated lipid and non-lipid risk factors (Grundy et al., 1999a) as well as to insulin resistance (Perseghin et al., 1996).

In the US, during the years $1997-1998,38.3 \%$ of adults 18 years and older reported no physical activity, $61.7 \%$ reported some activity but not enough to achieve recommended levels whereas, only $22.7 \%$ were reportedly engaged in light-moderate 
physical activity at least five times per week (AHA Statistical Update, 2004). Physical inactivity is more prevalent among women than men, among non-Hispanic Black men (46.0\%) and women $(57.1 \%)$ and, Hispanic men (49.9\%) and women $(57.1 \%)$ than nonHispanic White men (33.3\%) and women (38.9\%), among older than younger adults and among less affluent than the more affluent (AHA Statistical Update, 2004; USA Surgeon General's Report, 1996). A recent study of over 72,000 female nurses indicated that moderate-intensity physical activity, such as walking is associated with substantial reduction in risk of total and ischemic stroke when compared with physical activity done at an average or casual pace (Hu et al., 2000). In Florida, the percentage of adults who have no leisure-time physical activity $(70 \%-80 \%)$ has remained stable over the past 10 years (FLDH, 2001). However, there is no data on the prevalence of physical activity among the Caribbean Americans living in the US.

\section{Body Weight and Obesity}

Overweight is defined as a body mass index $(\mathrm{BMI}=$ weight in $\mathrm{kg}$ divided by the square of height in meters) of $25-29.9 \mathrm{~kg} / \mathrm{m}^{2}$ and obesity as BMI $\geq 30 \mathrm{~kg} / \mathrm{m}^{2}$ (Obesity: Class I BMI 30.0-34.9 kg/m²; Class II BMI $35.0-39.9 \mathrm{~kg} / \mathrm{m}^{2}$; Class III BMI $\geq 40.0$ $\mathrm{kg} / \mathrm{m}^{2}$ ). Over 108 million Americans age 20 years and older are overweight (over 55 million men and over 52 million women), with over 44 million being obese (over 18 million men and over 25 million women) (National Institutes of Health, 1998a,b; AHA Statistical Update, 2002; NCEP: ATP III, 2001). In addition, over 5 million children between the ages of 6-17 are overweight (based on the $95^{\text {th }}$ percentile of BMI values: CDC growth chart for the US, 2000). The annual total cost of obesity-related disorders in 
the US is over $\$ 100$ billion. As BMI values increase, the average blood pressure and total cholesterol levels increase, whereas the average HDL-C levels decrease (HP, 2010).

Individuals who are overweight or obese are at increased risk of all-cause mortality from CHD, stroke (Calle et al., 1999), high blood pressure (Blair et al., 1984), lipid disorders (Denke et al., 1993), type 2 diabetes mellitus (NCEP: ATP III, 2001; AHA Statistical Update, 2004), gallbladder disease, osteoarthritis, sleep apnea and other respiratory disorders and certain cancers (NIH, 1998a,b; HP, 2010; NCEP: ATP III 2001). Evidence from the Framingham Heart Study has indicated that obesity is strongly predictive of CHD and that risk for cardiovascular disease is increased when abdominal obesity is present (abdominal obesity defined as waist circumference $>102 \mathrm{~cm}[>40$ inches] in men and $>88 \mathrm{~cm}$ [ $>35$ inches] in women) $(\mathrm{NIH}, 1998 \mathrm{a}, \mathrm{b})$. Similarly, Han et al (1995) have shown that waist circumference, waist-to-hip ratio and BMI were positively correlated with measures of CHD risks such as blood pressure or blood lipids.

The age-adjusted prevalence of overweight in the US during 2001 increased from $55.9 \%$ in NHANES III (1988-1994) to $64.5 \%$ in NHANES IV (1999-2000). The prevalence of obesity also increased during this period from $22.9 \%$ to $30.5 \%$, whereas extreme obesity (Class III obesity) increased from $2.9 \%$ to $4.7 \%$ (AHA Statistical Update, 2004; Flegal et al., 2002). During this same period, the age-adjusted prevalence of overweight in the US was higher in non-Hispanic White men (67.4\%) compared to non-Hispanic Black men (60.7\%) but, higher in non-Hispanic Black women (77.3\%) compared to non-Hispanic White women (57.3\%). The age-adjusted prevalence of overweight in Mexican-American males and females was $74.7 \%$ and $71.9 \%$, respectively. The age-adjusted prevalence of obesity in non-Hispanic White men (27.3\%) and women 
(30.1\%) was lower compared to non-Hispanic Black men (28.9\%) and women (49.7\%), whereas the age-adjusted prevalence of obesity in Mexican-American males and females was $28.9 \%$ and $39.7 \%$, respectively (AHA Statistical Update, 2004; Flegal et al., 2002).

Other developed countries are experiencing similar increases and developing countries are showing increases in the incidence and prevalence of obesity and overweight as they become more affluent (Flegal et al., 2002; WHO, 2000b). The prevalence of obesity in Caribbean males is estimated to be $7-12 \%$ whereas the range in females is estimated to be $20-48 \%$ (Henry, 2001). In England, there is evidence showing higher age-adjusted prevalence of obesity (average 36.3\%) in Afro Caribbeans compared to Whites (average 28.7\%) (Dundas et al., 2001; Cappuccio et al., 1997). Singh and Siahpush (2002) have shown that the percentage of a composite migrant Black population (25.2\%) that was overweight was lower compared to their US-born counterparts $(38.4 \%)$. In Florida during the year 2002, more than $57 \%$ of the adult population was overweight, whereas $19.4 \%$ was obese (FLDH, 2001). More non-Hispanic Blacks (39.5\% and 32.3\%) were overweight and obese compared to non-Hispanic Whites (36.0\% and 18.0\%), respectively (FLDH, 2001). However, data relating to overweight and obesity differentials in foreign-born and US-born Afro Caribbeans in Florida is non-existent.

\section{Cigarette Smoking and Alcohol Consumption}

In the US, tobacco use is the leading preventable cause of death (FLDH, 2001). There is ample evidence to support the claim that cigarette smoking is a contributor to the risk for CHD and other forms of CVD (Kannel et al., 1980; Colditz et al., 1988; LaCroix, 1991; McBride, 1992; Jonas et al., 1992; Pyörälä et al., 1994). This relationship is dose 
related and smoke cessation will reduce the risk for CVD events within months of quitting (US Surgeon General Report, 1990). According to the American Heart Association (AHA Statistical Update, 2002), the risk of CHD is reduced up to $50 \%$ one year after smoke cessation and, within 15 years, the relative risk of dying from CHD of an ex-smoker approaches that of a lifetime non-smoker.

The age-adjusted prevalence of smoking in Americans 18 years and older is $25.2 \%(25,600,000)$ in males and $20.7 \%(22,600,000)$ in females putting them at increased risk for CHD and stroke (Health United States, 2001; AHA Statistical Update, 2004). The use of tobacco products in the year 2001 was higher in non-Hispanic Whites (31.3\%) compared to non-Hispanic Blacks (27.7\%) (AHA Statistical Update, 2004). A similar trend was seen in England where more Whites (31.2\%) were current smokers compared to Afro-Caribbeans (22.6\%) (Dundas et al., 2001; Cappuccio et al., 1997). There is also evidence showing that smoking prevalence is highest among those with 911 years of education $(35.4 \%)$ compared with those with more than 16 years of education $(11.6 \%)$ and is highest among individuals living below the poverty level (33.3\%) compared with other income groups (MMWR, Vol 48, No. 43, 1999; AHA Statistical Update, 2004). Singh and Siahpush, (2002) reported that the percentage of foreign-born Blacks who were current smokers was lower (10.4\%) compared to US-born Blacks $(29.3 \%)$.

Alcohol Consumption

There is a complex relationship (J-shaped) between alcohol consumption and CHD (Pearson et al., 1996). Individuals who consume three or more alcoholic beverages 
each day, total mortality increases with the total number of drinks per day, whereas the lowest mortality, a reduction in risk of approximately $30 \%$ to $50 \%$ occurs in those who consume one or two drinks per day (Bradley et al., 1993). In Florida, $6.4 \%$ of individuals reported heavy drinking (the consumption of $>2$ drinks/day for men and $>1$ drink/day for females during the past month). In the Miami-Miami Beach-Kendall section of Florida, 3.5\% of individuals reported heavy alcoholic beverage consumption (MMWR, 2004). There is limited data on the drinking habits of Afro Caribbean Americans.

\section{Bio-physiological Risk Factors for CHD}

\section{Blood Pressure and Hypertension}

High blood pressure (HBP) (hypertension) is traditional defined as systolic blood pressure (SBP) of $140 \mathrm{mmHg}$ or greater and/or diastolic blood pressure (DBP) of 90 $\mathrm{mmHg}$ or greater or current use of antihypertensive treatment $\left(6^{\text {th }}\right.$ Report of the Joint National Committee on Prevention, Detection, Evaluation and Treatment of High Blood Pressure [JNC VI], 1997). However, a recent report (JNC VII, 2003) has classified normal $\mathrm{BP}$ as $\mathrm{SBP}<120 \mathrm{~mm} \mathrm{Hg}$ and/or DBP $<80 \mathrm{~mm} \mathrm{Hg}$ and, has added a new category, designated pre-hypertension (SBP 120-139 mm Hg OR DBP 80-89 mm Hg) (Table 4). 
Table 4 - Classification of Blood Pressure for Adults Aged 18 Years and Older

\begin{tabular}{|c|c|c|}
\hline BP Classification & Systolic BP, $\mathrm{mm} \mathrm{Hg}^{*}$ & Diastolic BP, $\mathrm{mm} \mathrm{Hg}^{*}$ \\
\hline Normal & $<\mathbf{1 2 0}$ and & $<\mathbf{8 0}$ \\
\hline Pre-hypertension & $\mathbf{1 2 0 - 1 3 9}$ or & $\mathbf{8 0 - 8 9}$ \\
\hline Stage 1 Hypertension & $\mathbf{1 4 0 - 1 5 9}$ or & $90-99$ \\
\hline Stage 2 Hypertension & $\geq \mathbf{1 6 0}$ or & $\geq \mathbf{1 0 0}$ \\
\hline
\end{tabular}

Adapted from JNC VII, 2003).

*Treatment determined by highest BP category.

Hypertension is a major risk factor for $\mathrm{CHD}$, stroke, kidney failure and heart failure (JNC VI, 1997). Systolic blood pressure is now being recognized as a more potent predictor of CHD than DBP, especially in older adults (Kannel et al., 1980; Systolic Hypertension in the Elderly Program [SHEP], 1991). About 50 million persons, in the US, age 6 years and older have high blood pressure (AHA Statistical Update, 2002; NHANES III, 1988-1994). Of those with HBP, 30\% are unaware they have it, 34\% controlled with medication, $25 \%$ are on medication but do not have it under control and, $11 \%$ are not on medication (JNC VII, 2003). High blood pressure is associated with physical inactivity, high salt and alcohol intakes, and low intakes of potassium and calcium (FLDH, 2001). Control of HBP through weight loss, dietary, behavior or medication changes has been shown to be very effective in reducing the risk of CHD (FLDH, 2001). Individuals with lower educational and income levels tend to have higher blood pressure compared to those with higher educational and income levels (AHA Statistical Update, 2002).

The overall death rate from HBP in the US in 2001 was $16.5 / 100,000$ population. However, death rates for African American males and females were higher (47.8 and $38.9 / 100,000$ population, respectively), compared to White males and females (13.7 and 
13.4/100,000 population, respectively) (AHA Statistical Update, 2004). African Americans develop HBP earlier in life and their average blood pressures are higher than Whites. Thus, African Americans have 1.3 times greater rate of non-fatal stroke, 1.8 times greater rate of fatal stroke, 1.5 times greater rate of heart disease death and 4.2 times greater rate of end-stage kidney disease compared to Whites (JNC VI, 1997). The death rates from hypertensive disease in the Caribbean during 1987-1991 were much higher than those seen in the US and Canada during the same period (Sinha, 1995).

The age-adjusted prevalence of HBP in individuals 20 years and older in 2001, in the US, was higher in non-Hispanic Black males (41.6\%) and females (44.7\%) compared to non-Hispanic White males (32.2\%) and females (29.5\%) (AHA Statistical Update, 2004). Data from England also indicate that Afro-Caribbeans have higher blood pressure values compared to Whites (Lemic-Stojcevic et al., 2001; Cappuccio et al., 1997), however, data in the US (Singh and Siahpush, 2002) showed the percentage of foreignborn Blacks who had high blood pressure was lower (16.8\%) compared to US-born Blacks (25.5\%). Cooper et al (1997a) investigating hypertension prevalence in individuals of African Ancestry in the Caribbean and the US also showed the prevalence of hypertension was lower in the Caribbean (26\%) compared to the US (33\%). There is insufficient data on blood pressure differentials in foreign-born and US-born Afro Caribbean Americans in Florida, however.

\section{Fasting Blood Glucose, Metabolic Syndrome and Diabetes}

Impaired fasting blood glucose level (110-125 mg/dL) is an indicator of insulin resistance and is generally accompanied by other metabolic risk factors (Tripathy et al., 
2000; NCEP: ATP III, 2001). Some individuals with impaired fasting blood glucose will eventually develop type 2 diabetes (Lindahl et al., 1999), which further enhances the risk for CHD. Type 2 diabetes is the epitome of the metabolic syndrome, characterized by abdominal obesity, atherogenic dyslipidemia (elevated triglyceride, small low-density particles, low high-density cholesterol), elevated blood pressure, insulin resistance, prothrombotic and proinflammatory conditions (Table 5) (NCEP: ATP III, 2001). This disorder is associated with increased risk of CHD and occurs in $24 \%$ of the US adult population (Ford et al., 2002).

Table 5 - Clinical Identification of the Metabolic Syndrome*

\begin{tabular}{|c|c|}
\hline Risk Factors & Defining Level \\
\hline Abdominal Obesity & Waist circumference $\dagger$ \\
Men & $>40 \mathrm{in}(>102 \mathrm{~cm})$ \\
Women & $>35 \mathrm{in}(88 \mathrm{~cm})$ \\
\hline Triglycerides & $\geq 150 \mathrm{mg} / \mathrm{dL}$ \\
\hline HDL Cholesterol & $<40 \mathrm{mg} / \mathrm{dL}$ \\
Men & $<50 \mathrm{mg} / \mathrm{dL}$ \\
Women & $\geq 130 / 85 \mathrm{~mm} \mathrm{Hg}$ \\
\hline Blood Pressure & $\geq 110 \mathrm{mg} / \mathrm{dL}$ \\
\hline Fasting Blood Glucose &
\end{tabular}

* The ATP III panel did not find adequate evidence to recommend routine measurement of insulin resistance (e.g., plasma insulin), proinflammatory state (e.g., high-sensitivity C-reactive protein), or prothrombotic state (e.g., fibrinogen or PAI-1) in the diagnosis of the metabolic syndrome.

† Some male persons can develop multiple metabolic risk factors when the waist circumference is only marginally increased, e.g., $37-39$ in (94-102 cm). Such persons may have a strong genetic contribution to insulin resistance. They should benefit from changes in lifestyle habits, similarly to men with categorical increases in waist circumference.

Source: NCEP: ATP III, 2001 


\section{Diabetes Mellitus}

A sustained blood glucose level equal to or greater than $126 \mathrm{mg} / \mathrm{dL}$ is indicative of diabetes mellitus (Gavin et al., 1998). Diabetes mellitus (DM) is a multi-factorial condition caused by inherited and/or deficiency in insulin production, by the $\beta$-cells of the pancreas or, by ineffectiveness of the insulin produced. Sustained elevation of blood glucose and abnormalities in the metabolism of carbohydrates, fats, and proteins can lead to tissue and organ dysfunction and damage, especially in eyes, kidneys, nerves, and blood vessels. Type $1 \mathrm{DM}$ (insulin-dependent DM) (5\% of diabetics) is characterized by absolute lack of insulin whereas, Type 2 DM (non insulin-dependent DM) (90\% of diabetics) is characterized by insulin resistance and defects in insulin action. There are other types (5\%) of DM due to pregnancy, DM secondary to specific genetic syndromes, drugs, malnutrition, infections and other medical conditions (Gavin et al., 1998; CDC Diabetes Factsheets, 2000). Individuals with undiagnosed type $2 \mathrm{DM}$ are at increased risk for stroke, CHD, and peripheral vascular disease compared to the non-diabetic population (American Diabetes Association [ADA], 2002). Obesity, impaired glucose tolerance, habitual physical inactivity, stress, race/ethnicity, family history of DM, prior history of gestational DM, hypertension, high-density lipoprotein cholesterol $\leq 35 \mathrm{mg} / \mathrm{dL}$ and/or a triglyceride level $\geq 250 \mathrm{mg} / \mathrm{dL}$, and advancing age are recognized risk factors for type 2 DM (CDC Diabetes Factsheets, 2000; ADA, 2002).

The prevalence of DM in adults globally was estimated to be $4.0 \%$ (135 million individuals) in 1995 and, it is predicted to increase to $5.4 \%$ (300 million individuals) by the year 2025 (HP 2010). In 2001, the prevalence of DM in the US was over 11 million $(5.5 \%)$ with nearly 6 million $(2.9 \%)$ of these individuals unaware that they have elevated 
blood glucose (CDC Diabetes Factsheets, 2000; AHA Statistical Update, 2004). Over 600,000 individuals are diagnosed with DM each year. Diabetes accounts for over 200,000 deaths and \$18.9 million in ambulatory visits annually (CDC Diabetes Data Sheet, 2000). In the year 2001, the age-adjusted prevalence of DM in individuals 20 years and older for non-Hispanic White males (5.5\%) and females (4.7\%) was lower compared to non-Hispanic Black males (7.6\%) and females (9.5\%), with Hispanic males $(8.1 \%)$ and females (11.4\%) having even higher prevalence (NHANES III, 1988-94; AHA Statistical Update, 2004). A similar trend has been observed in Afro-Caribbeans in London who had significantly higher (18.3\%) glycosylated hemoglobin (HbAlc) levels compared to Whites (7.0\%) (Lemic-Stojcevic et al., 2001). Also, Cooper et al (1997) investigating rates of non-insulin dependent diabetes mellitus (NIDDM) in individuals of African Ancestry in Nigeria, the Caribbean, the US and the UK showed that the rates of NIDDM were $2 \%, 9 \%, 11 \%, 11 \%$, respectively. The prevalence of DM in the Caribbean is estimated to range from $10-15 \%$ (Sinha, 1995).

In Florida, trends in DM mortality rate, showed an increase from 11.5/100,000 population in 1950 to $13.3 / 100,000$ population in 2000 , with DM mortality rates being much higher in non-Hispanic Blacks (50\%) compared to non-Hispanic Whites (18\%) (FLDH, 2001). The annual economic cost of DM in Florida in 2001 was over $\$ 6$ billion (FLDH, 2001). There is no published data on the blood glucose levels in Caribbean Americans. 


\section{Lipid Risk Factors - Serum Lipids}

Observations about the role of lipids in cardiovascular disease were documented as early as the mid to late 1800 s. A century later, numerous clinical studies documented increased amounts of blood cholesterol in atherosclerotic vessels as well as in the blood of survivors of myocardial infarction. Studies in the 1950s demonstrated that serum cholesterol levels showed a dose-response relationship to CVD death rates (Criqui and Beatrice, 1998).

\section{Total Cholesterol}

In the US, more than 50 million adults have elevated blood cholesterol levels that need medical attention (HP 2010; Sempos et al., 1993), whereas over 104 million have blood cholesterol levels that are higher than desirable $(200 \mathrm{mg} / \mathrm{dL}$ and higher) (NCEP: ATP III, 2001; AHA Statistical Update, 2004). As blood cholesterol rises, so does risk for CHD. In the Multiple Risk Factor Intervention Trial (MRFIT), the age-adjusted death rate from CHD, in men, increased from 3.5/1,000 at a TC of $140 \mathrm{mg} / \mathrm{dL}$ to about 5.0/1,000 at a TC of $200 \mathrm{mg} / \mathrm{dL}$ (Martin et al., 1986). Data from the Seven Countries Study also supported this by showing an exponential relationship between CHD and higher levels of TC (Verschuren et al., 1995). When other risk factors such as high blood pressure and smoking are present, the risk will increase greatly. An individual's blood cholesterol levels are also affected by age, gender, heredity and diet (AHA Statistical Update, 2004; NCEP: ATP III, 2001). Mortality from CHD has been shown to begin with a blood cholesterol level as low as $180 \mathrm{mg} / \mathrm{dl}$, increasing as blood cholesterol increases, especially above $200 \mathrm{mg} / \mathrm{dL}$ (Stamler et al., 1986). Evidence from research, in the 1980s, 
showed for the first time that lowering high blood cholesterol significantly reduces the risk for heart attacks and heart attack deaths.

In the year 2001, the prevalence of TC (greater than $200 \mathrm{mg} / \mathrm{dL}$ ) was lower in non-Hispanic Black males (37.3\%) and females (46.4\%) compared to non-Hispanic White males (51.0\%) and females (53.6\%) (AHA Statistical Update, 2004). There is now evidence indicating that a $10 \%$ decrease in total cholesterol levels may result in an estimated 30\% reduction in the incidence of CHD (MMWR, 2000).

Similar trends in serum TC and HDL-C levels are seen in Afro-Caribbeans who live in London (Lemic-Stojcevic et al., 2001). Lemic-Stojcevic et al. (2001) showed that TC $(6.0 \mathrm{mmol} / \mathrm{L})$ and TG $(1.77 \mathrm{mmol} / \mathrm{L})$ levels in Whites were significantly $(\mathrm{p}<0.0001)$ higher than in Afro-Caribbeans (5.55 mmol/L and 1.39 respectively). However, HDL-C levels $(1.59 \mathrm{mmol} / \mathrm{L})$ in Afro-Caribbeans were significantly higher than values in Whites $(1.50 \mathrm{mmol} / \mathrm{L})$.

Lifestyle changes that can help prevent or lower high blood cholesterol include smoking cessation, a diet low in saturated fat and cholesterol, increasing physical activity and reducing excess weight (HP, 2010; NCEP: ATP III, 2001). The Framingham study (LaRosa et al., 1990) has shown that a treatment regime that lowers an individual's blood cholesterol by $1 \%$ can be translated into a $2 \%$ reduction in CHD risk. It is suggested that differences in lipid profile may account for some of the ethnic differences in CHD mortality rates in the US population (Whitty et al., 1999). There is no standardized data on the serum lipid levels of Caribbean Americans. These values may be different since Caribbean Americans in the US may have different dietary and behavioral habits compared to Afro-Caribbeans who live in England. 
The more risk factors one has, the greater the chance of developing CHD and stroke. Also, the greater the level of each risk factor, the greater the risk. Even though everyone with a serum cholesterol level greater than $240 \mathrm{mg} / \mathrm{dL}$ is considered at high risk, a person with a TC of $300 \mathrm{mg} / \mathrm{dL}$ is at a much greater risk than someone with a total cholesterol of $240 \mathrm{mg} / \mathrm{dL}$ (AHA Statistical Update, 2002). In the absence of these traditional risk factors, death from cardiovascular causes is relatively rare (Beaglehole et al., 2001).

\section{Low-density Lipoprotein Cholesterol}

High levels of Low-Density Lipoprotein Cholesterol (LDL-C), an atherogenic lipoprotein that contains most of the body's cholesterol, is a powerful risk factor for CHD (NCEP: ATP 111, 2001). There is evidence indicating that individuals who have very low levels of LDL-C throughout life due to familial hyperbetalipoproteinemia have an extended life span (NCEP: ATP III, 2001; Glueck et al., 1976). In individuals with the genetic forms of hypercholesterolemia advanced coronary atherosclerosis and premature CHD occur even in the complete absence of other risk factors (Brown and Goldstein, 1986; Austin et al., 2000a; NCEP: ATP III, 2001). These disorders provide the strongest evidence that LDL-C is a powerful atherogenic lipoprotein (NCEP: ATP III, 2001). The Framingham Heart Study (Wilson et al., 1998), the Multiple Risk Factor Intervention Trial (MRFIT) (Stamler et al., 1986), and the Lipid Research Clinics (LRC) trial (Lipid Research Clinics Program 1984a,b) have shown a direct relationship between LDL-C (or TC) and the rate of new onset of CHD in men and women who were initially free of CHD. Any LDL-C levels above $100 \mathrm{mg} / \mathrm{dL}$ appear to be atherogenic (NCEP:ATP III, 
2001). Atherosclerosis proceeds at a significant rate when LDL-C is between 130-159 $\mathrm{mg} / \mathrm{dL}$ whereas above $160 \mathrm{mg} / \mathrm{dL}$ it is markedly elevated. These relationships have been confirmed by the log-linear relationship between serum cholesterol levels and CHD risk observed in many populations (Law et al., 1994b; Law, 1999).

In the US, the mean LDL-C level of adults 20 years and older is $127 \mathrm{mg} / \mathrm{dL}$. In the year 2001, the prevalence of LDL-C $130 \mathrm{mg} / \mathrm{dL}$ and higher was higher in nonHispanic White males (49.6\%) and females (43.7\%) compared to non-Hispanic Black males (46.3\%) and females (41.6\%) (AHA Statistical Update, 2004). There is no published data on LDL-C levels in foreign-born and US-born Afro Caribbeans.

\section{Serum Triglycerides}

There is evidence, albeit inconsistent indicating a positive relationship between serum triglyceride (TG) levels and incidence of CHD (Austin et al., 1998; Assmann et al., 1998a; NCEP: ATP III, 2001). However, the NCEP: ATP III (2001) Guidelines give increased weight to elevated TG in cholesterol management as a marker for atherogenic remnant lipoproteins and as a marker for other lipid and non-lipid risk factors in the metabolic syndrome.

\section{High-density Lipoprotein Cholesterol}

There is strong epidemiological evidence indicating that low levels of HighDensity Lipoprotein Cholesterol (HDL-C) increase CHD mortality and morbidity (Wilson et al., 1998; Gordon et al., 1989; NCEP: ATP III, 2001). Levels of HDL-C above $60 \mathrm{mg} / \mathrm{dL}$ are considered desirable and cardio-protective, whereas levels below $40 \mathrm{mg} / \mathrm{dL}$ 
are considered a risk factor for CHD and stroke (NCEP: ATP III, 2001; AHA Statistical Update, 2004). Individuals with HDL-C $<40 \mathrm{mg} / \mathrm{dL}$ and $\mathrm{TG}>150 \mathrm{mg} / \mathrm{dL}$ are at increased risk of CHD. Indeed, males with HDL-C levels $37 \mathrm{mg} / \mathrm{dL}$ or lower and, females with HDL-C levels $47 \mathrm{mg} / \mathrm{dL}$ or lower are at the highest risk regardless of their TC levels (AHA Statistical Update, 2004). However, males with HDL-C levels $53 \mathrm{mg} / \mathrm{dL}$ or greater and, females with HDL-C levels $67 \mathrm{mg} / \mathrm{dL}$ or greater, are at low risk for CHD even if they have elevated TC levels (AHA Statistical Update, 2004).

In the NHANES III (1988-1994) study, the mean level of HDL-C for adults 20 years and older was $50.7 \mathrm{mg} / \mathrm{dL}$. In the year 2001, the prevalence of low HDL-C ( $<40$ $\mathrm{mg} / \mathrm{dL}$ ) was higher in males $(39.0 \%)$ compared to females $(14.9 \%)$. Also, the prevalence of low HDL-C was lower in non-Hispanic Black males (24.3\%) and females (13.0\%) compared to non-Hispanic White males (40.5\%), females (14.5\%) and Mexican American males (40.1\%) and females (18.4\%) (AHA Statistical Update, 2004).

There is also evidence indicating that a $1 \%$ decrease in HDL-C is associated with a 2-3\% increase in CHD risk (Gordon et al., 1989). The mechanism for this association is suggested to be through a reverse cholesterol transport pathway. Other suggested mechanisms include inhibition of lipid peroxidation (Mackness and Durrington, 1995), cellular adhesion (Barter, 1997) and/or platelet activation (Sugatani et al., 1996). In the US, low levels of HDL-C rather than high LDL-C levels are the most prevalent finding (Rubins et al., 1995). Blood levels of HDL-C can be influenced by elevated serum TG, overweight and obesity, physical inactivity, cigarette smoking, very high carbohydrate intakes (more than $60 \%$ of total energy intake), type 2 diabetes, certain drugs (beta- 
blockers, anabolic steroids, progestational agents) and genetic factors. Table 6 shows the classification of serum TC, LDL-C, TG and HDL-C levels (NCEP: ATP III, 2001).

Table 6 - NCEP: ATP III Classification of Total Cholesterol, Low-density Lipoprotein Cholesterol, Triglycide and High-density Lipoprotein Cholesterol

\begin{tabular}{|c|c|c|c|c|c|c|c|}
\hline \multicolumn{2}{|c|}{$\begin{array}{c}\text { Total Cholesterol } \\
\text { (mg/dL) }\end{array}$} & \multicolumn{2}{c|}{$\begin{array}{c}\text { LDL Cholesterol } \\
\text { (mg/dL) }\end{array}$} & \multicolumn{2}{c|}{$\begin{array}{c}\text { Triglycerides } \\
\text { (mg/dL) }\end{array}$} & \multicolumn{2}{|c|}{$\begin{array}{c}\text { HDL Cholesterol } \\
\text { (mg/dL) }\end{array}$} \\
\hline$<200$ & Desirable & $<100$ & Optimal & $<150$ & Normal & $\geq 60$ & Desirable \\
\hline $200-239$ & $\begin{array}{c}\text { Borderline } \\
\text { High }\end{array}$ & $100-129$ & $\begin{array}{c}\text { Near } \\
\text { optimal } \\
\text { above } \\
\text { optimal }\end{array}$ & $150-199$ & $\begin{array}{c}\text { Border } \\
\text { line } \\
\text { High }\end{array}$ & & \\
\hline$\geq 240$ & High & $130-159$ & $\begin{array}{c}\text { Borderline } \\
\text { High }\end{array}$ & $200-499$ & High & $<40$ & Undesirable \\
\hline & $160-189$ & $\begin{array}{c}\text { High } \\
\text { High }\end{array}$ & $\geq 500$ & $\begin{array}{c}\text { Very } \\
\text { High }\end{array}$ & & \\
\hline & & $\geq 190$ & Very High & & & & \\
\hline
\end{tabular}

Source: NCEP: ATP III, 2001

\section{Emerging Biochemical Risk Factors for CHD}

The behavioral/environmental and physiological risk factors for $\mathrm{CHD}$ (increasing age, cigarette smoking, elevated blood pressure, blood sugar, LDL-C, TC, low HDL-C), have been shown to be positively associated with the development of CHD (Greenland et al., 2003; Ridker, 2003a; Beaglehole et al., 2001; AHA Statistical Update,2002; NCEP: ATP III, 2001). However, these major risk factors can explain only about $70 \%$ of the variability in CHD risk in the US population (NCEP: ATP III, 2001; Ridker, 2003a). Thus, the intensive research to identify new (emerging) risk factors that will enhance predictive power in individuals with and without CHD is important (ATP III, 2001). These emerging risk factors are listed in Table 6 (Ross, 1999; NCEP: ATP III, 2001). 
Of the emerging risk factors listed in Table 7, inflammatory markers, such as high-sensitivity C-reactive protein (hs-CRP) has received the most attention (Ridker, 1999). However, these emerging risk factors must be evaluated against the following criteria used to identify the major risk factors:

Table 7 - Emerging Risk Factors for Coronary Heart Disease

Lipid Factors
- TC/HDL-C ratio*
- Lipoprotein (a)
- Lipoprotein Remnants
- - Small LDL Particles
- Apolipoprotein B
- Apolipoprotein A1

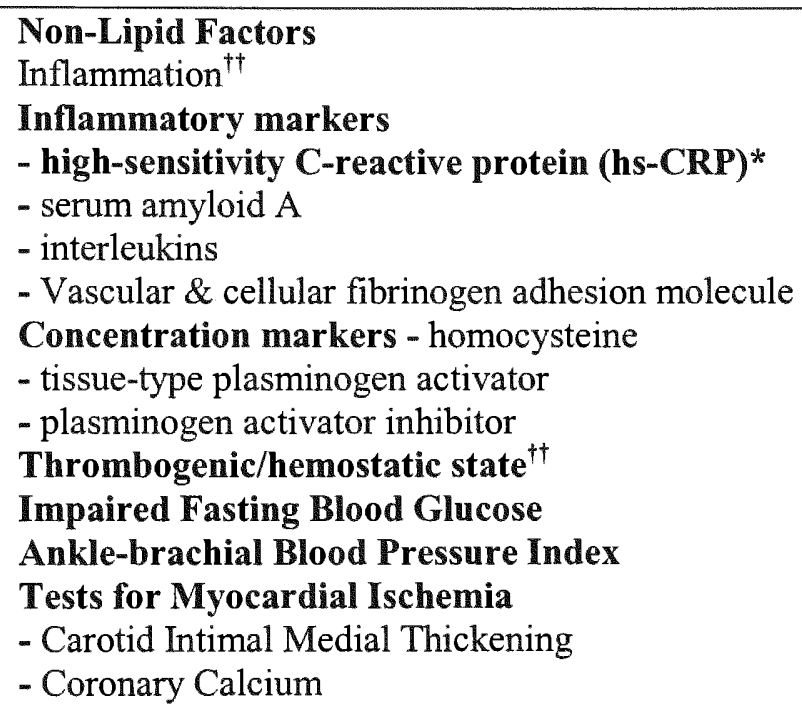

* Emerging risk factors investigated in the present study.

$\dagger$ Emerging evidence has indicated that chronic arterial inflammation as a risk factor for major coronary events.

$\dagger \dagger$ This risk is inferred from observations that antiplatelet drugs and anticoagulants have been shown to reduce risk for CHD.

Adapted from Ridker, 1997. Association of hemostatic and thrombotic factors with

cardiovascular risk. In: Schafer AI, ed. Molecular Mechanisms of Hypercoagulable States.

Austin, TX: Landes Boiscience; 1997; ATP III, 2001.

\section{Laboratory or clinical measurement must be widely available, well}

standardized, inexpensive, have accepted population-reference values, and be relatively stable biologically (Ridker, 1999; NCEP: ATP III, 2001). The efficacy and 
clinical use of other emerging markers of CHD has been inconsistent (Tate et al., 1998;

Ridker, 1999; Angleton et al., 1989; Andreotti and Kluft, 1991; Ridker, 1999). However, the testing for hs-CRP has provided consistent and reliable results (Macy et al., 1997; Ledue et al., 1997), and standards for the assessment of hs-CRP have been set by the World Health Organization (WHO, 1987).

\section{Significant predictive power that is independent of the other major risk}

factors. It is not sufficient that a particular marker of risk for CHD has predictive value in univariate analysis, since it may be confounded by other traditional risk factors (Ridker, 1999). Information from both the Physician Health Study (Ridker et al., 1998c) and the Women's Health Study (Ridker et al., 2000) have indicated that the predictive power of hs-CRP was significantly higher than those associated with the traditional biochemical markers of risk (TC, HDL-C, LDL-C, Lp(a), homocysteine, apolipoproteins $\mathrm{AI}$ and B) for CHD. In univariate analysis of future cardiovascular events hs-CRP was the strongest predictor of future risk (relative risk $[R R]=4.4 ; 95 \% \mathrm{CI}, 2.2-8.9$ ) whereas the LDL-C (well-established marker for $\mathrm{CHD})$ had a lesser predictive value $(\mathrm{RR}=2.4$; $95 \%$ CI, 1.3-4.6) for future cardiovascular events (Figure 3). However, when other risk factors for CHD (obesity, hypertension, diabetes, family history of CHD) were accounted for in multivariate analysis, only hs-CRP and TC/HDL-C ratio had independent predictive value (Figure 2). 
Figure 2 - Relative Risk of Future Cardiovascular Events

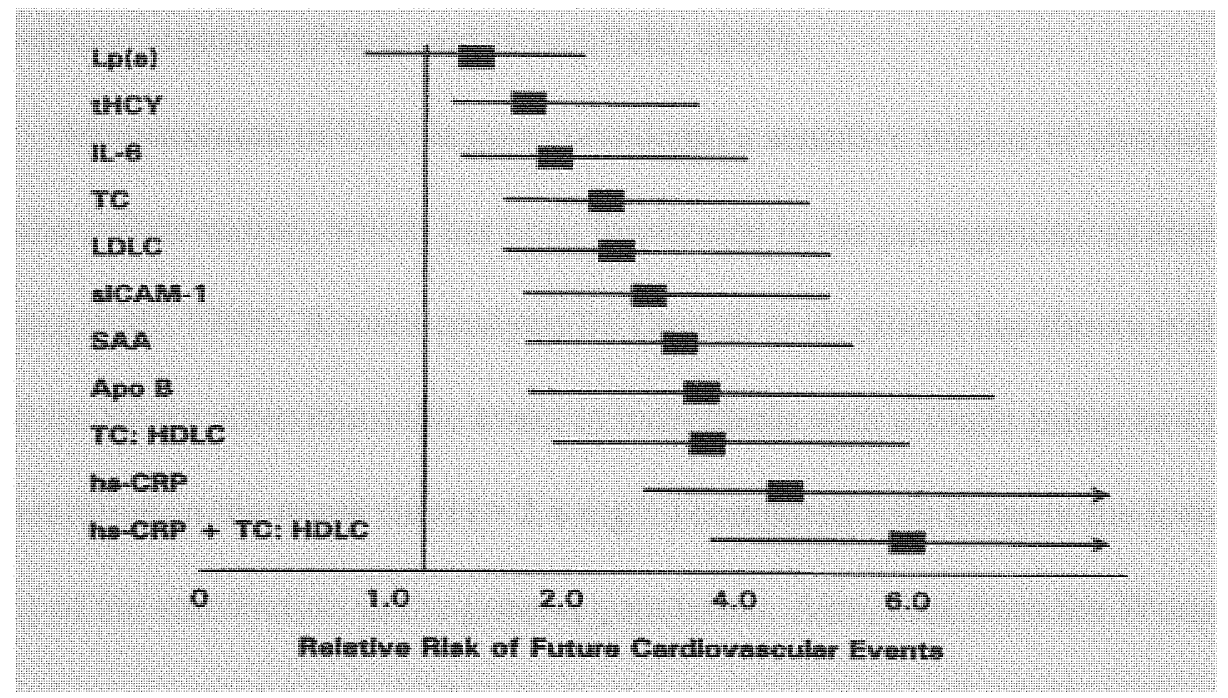

Relative Risk (RR) for future cardiovascular events among apparently healthy women in the WHS according to base line values of several biochemical markers. For consistency, risk estimates (1) and 95\% CIs (lines) are computed for those in the top compared with the bottom quartile for each marker. Lp(a), lipoprotein (a); tHCY, total homocysteine; IL-6, interleukin-6; sICAM-1, soluble intercellular adhesion molecule-1; LDLC, low-density lipoprotein cholesterol; SAA, serum amyloid A; Apo B, apolipoprotein B; HDLC, high-density lipoprotein cholesterol. Source: Ridker et al, 2000, with permission.

High-sensitivity CRP is highly stable over long periods of time and standardized, reproducible and automated analytical procedures for its measurement are available thus, it has become the most widely studied inflammatory bio-marker (Rifai and Ridker, 2002; Rifai et al., 1999). The plasma half-life of hs-CRP is approximately 19 hours and, is identical under all conditions (Koenig et al., 1999). Thus, the hepatic synthesis rate of hs-CRP is the sole determinant of its plasma concentration (Vigushin et al., 1993). Also, the intrinsic biological properties of hs-CRP, as an acute-phase 
substance, are favorable for its use as a sensitive quantitative systemic marker of the acute-phase response (Pepys and Hirschfield, 2003).

Measurement of CRP is now considered a sensitive marker of chronic low-grade vascular inflammation, which has been implicated in the pathogenesis of atherosclerosis (Koenig et al., 1999). A meta-analysis by Danesh et al. (2000) has shown a relative risk of 2.0 for future atherothrombic event in individuals with a single initial hs-CRP concentration in the upper third $(>2.4 \mathrm{mg} / \mathrm{L})$ compared with those in the lower third $(<1$ $\mathrm{mg} / \mathrm{L}$ ) of the distribution in the general population. Also, data from the Women's Health Study demonstrated that a single non-fasting measurement of hs-CRP (a) is a stronger predictor of future cardiovascular event compared to LDL-C, (b) identifies at-risk individuals with low LDL-C, (c) adds to the prognostic information of the currently used Framingham risk algorithm and, (d) identifies a higher risk for cardiovascular events in individuals with the metabolic syndrome (Ridker, 2003; Ridker et al., 2002; Ridker, 2003a). It is suggested that these findings are in strong agreement for screening of hsCRP to improve risk assessment in the primary prevention population (Ridker, 2003a).

\section{Preferably, but not necessarily, modification of the risk in clinical trials}

will have shown reduction in risk. Although there are no currently available therapies that specifically reduce hs-CRP levels, there is evidence showing a positive association between baseline hs-CRP levels and BMI, with weight loss lowering hs-CRP levels (Danish et al., 1999). Weight loss in obese elderly individuals has also been shown to reduce levels of acute phase proteins, such as hs-CRP (Calles-Escandon et al., 1996; Tisi et al., 1997). Tisi et al. (1997) investigated 49 individuals with intermittent claudication. 
They found that both serum hs-CRP and amyloid A were significantly reduced after 3 to 6 months of regular physical activity. Physical activity (Ford, 2002) and moderate alcohol consumption (Imhot et al., 2001) have also been associated with lowering of baseline hsCRP levels. There are results from clinical studies indicating that the relative efficacy of low-dose aspirin and 3-hydroxy-3-methylglutaryl coenzyme A reductase inhibitors (statins) may be greater in individuals with evidence of underlying inflammation as assessed by hs-CRP (Ridker et al., 1997; Ridker et al., 1998). Statins have also been shown to reduce the risk of future cardiovascular events to the same extent in individuals with normal and/or elevated LDL-C levels but, with baseline hs-CRP levels above the median value (Ridker et al., 2001). In the secondary prevention Cholesterol and Recurrent Events (CARE) trial, the magnitude of preventive benefit associated with pravastatin treatment was $55 \%$ in individuals with high hs-CRP concentrations compared with $30 \%$ in individuals with low hs-CRP concentrations (Ridker et al., 1998; Ridker, 2003a). In the primary prevention large-scale statin trial (Air Force/Texas Coronary Atherosclerosis Prevention Study), lovastatin treatment reduced the risk of first acute coronary events by $37 \%$ in individuals with average total and LDL-C concentrations and below average HDL-C concentrations. Also, after one year of lovastatin treatment, hsCRP concentrations were reduced by $15 \%$ compared to the untreated group. There were also large reductions in CHD event rates in the lovastatin group with low LDL-C/high hsCRP, high LDL-C/low hs-CRP and, high LDL-C/high hs-CRP concentrations compared to the placebo group, suggesting that apparently healthy individuals with low LDL-C maybe at increased risk of CHD events based on the measurements of hs-CRP (Ridker et al., 2001; Ridker, 2003a). It has been suggested that measurement of hs-CRP may thus, 
have important roles in monitoring preventive therapies or an indication for prophylactic antiatherosclerotic therapy in otherwise apparently healthy individuals (Ridker, 1999; Rohde et al., 1999; Pepys and Hirschfield, 2003).

\section{High-sensitivity C-reactive Protein and Coronary heart Disease}

Numerous prospective epidemiological studies in individuals without a history of CHD have consistently shown that a single, non-fasting measure of CRP to be a powerful predictor of future first coronary event (Figure 4) (Kuller et al., 1996; Ridker et al., 1997; Tracy et al., 1997b; Ridker et al., 1998a,b; Koenig et al., 1999; Ridker et al., 2000; Roivainen et al., 2000; Mendall et al., 2000; Danesh et al., 2000; Gussekloo et al., 2000; Packard et al., 2000; Lowe et al., 2001; Ridker et al., 2001; Rost et al., 2001; Pradham et al., 2002; Albert et al., 2002; Sakkinen et al., 2002). This has been observed across different populations, such as smokers enrolled in the Multiple Risk Factor Intervention Trial (MRFIT) (Kuller et al., 1996), elderly individuals in the Cardiovascular Health Study (Tracy et al., 1997b), postmenopausal women in the Women's Health Study (Ridker et al., 2000; Ridker et al., 1998b) and in Europe, individuals in the MONItoring trends and determinants in CArdiovascular disease (MONICA) Augsberg study (Koenig et al., 1999), the Helsinki Heart Study (Roivainen et al., 2000) and the British Regional Practice study (Danesh et al., 2000) (Figure 3). 
Figure 3 - Prospective Studies of hs-CRP as a Marker for Future Cardiovascular Events in Populations of Apparently Healthy Men and Women (Without Known CHD).



For Consistency across Studies, Risk Estimates ( $)$ and $95 \%$ CIs (lines) are calculated as comparison of top versus bottom quartile within each study population. CHD indicates coronary heart disease; $\mathrm{MI}$, myocardial infarction; $\mathrm{PAD}$, pulmonary artery disease; $\mathrm{CV}$, cardiovascular; MRFIT, Multiple Risk Factor Intervention Trial; PHS, Physicians' Health Study; CHS, Cardiovascular Health Study; RHPP, Rural Health Promotion Project; WHS, Women's Health Study; MONICA, MONItoring trends and determinants In CArdiovascular disease; HELSINKI, Helsinki Heart Study; CAERPHILLY, Caerphilly Heart Study; BRHS, British Regional Heart Study; LEIDEN, Leiden Heart Study; SPEEDWELL, Speedwell Heart Study; WOSCOPS, West of Scotland Coronary Prevention Study; AFCAPS, Air Force Coronary Atherosclerosis Prevention Study; FHS, Framingham Heart Study; WHI, Women's Health Initiative; and HHS, Honolulu Heart Study.

Source: Ridker, 2003 with permission.

From these same studies there is evidence indicating that serum hs-CRP levels are increased many years in advance of the first coronary and cerebrovascular events in healthy (Ridker et al., 1997; Ridker et al., 1998b; Koenig et al., 1999) and high-risk individuals (Tracy et al., 1997b; Kuller et al., 1996). In two of these studies (Ridker et al., 1998a, 2000), hs-CRP was shown to be a powerful indicator of future cardiovascular 
events even among subgroups of women with no history of hyperlipidemia, hypertension, smoking, diabetes or family history of CHD.

Ridker et al (2000) have shown that it is possible to predict future risk for cardiovascular events (including $\mathrm{MI}$, stroke, coronary revascularization) even in individuals with LDL-C values $<130 \mathrm{mg} / \mathrm{dL}$, a level stated by the NCEP: ATP III (2001) panel to be desirable. Riker et al (2002) evaluated hs-CRP levels in over 29,000 women (45 years and older) in the Women's Health Study. They showed that women in the high hs-CRP/low LDL-C group were at higher absolute risk compared to those in the low hsCRP/high LDL-C group (Figure 4). They suggested that these individuals (high hsCRP/low LDL-C) would normally not be considered at risk for preventive treatment in the clinical setting, thus, the importance of measuring hs-CRP along with LDL-C levels (Ridker et al., 2002). Also, hs-CRP levels were found to add substantial prognostic information to the Framingham risk score and the LDL-C categories of $<130 \mathrm{mg} / \mathrm{dL}, 130$ to $160 \mathrm{mg} / \mathrm{dL}$, and $>160 \mathrm{mg} / \mathrm{dL}$ used in the current ATP III guidelines (Figure 5) (NCEP: ATP III, 2001; Ridker, 2003a).

Ridker et al (2002) have shown that hs-CRP levels can either be interpreted in terms of population-based quintiles (graph on the left of Figure 6) or clinical cut-points (graph on right of figure 6). It is suggested that the graph on the right of figure 6 in which hs-CRP levels of $<1,1-3$, and $>3 \mathrm{mg} / \mathrm{L}$ represent low-, moderate-, and high-risk groups, respectively, may have more clinical application (Ridker et al. 2002). Both the American Heart Association and the Centers for Disease Control and Prevention now endorse these clinical cut points for hs-CRP evaluation (Pearson et al., 2003; Ridker, 2003a). 
Figure 4 - Event-free survival among women with hs-CRP and LDL-C levels above or below the median for the study population.

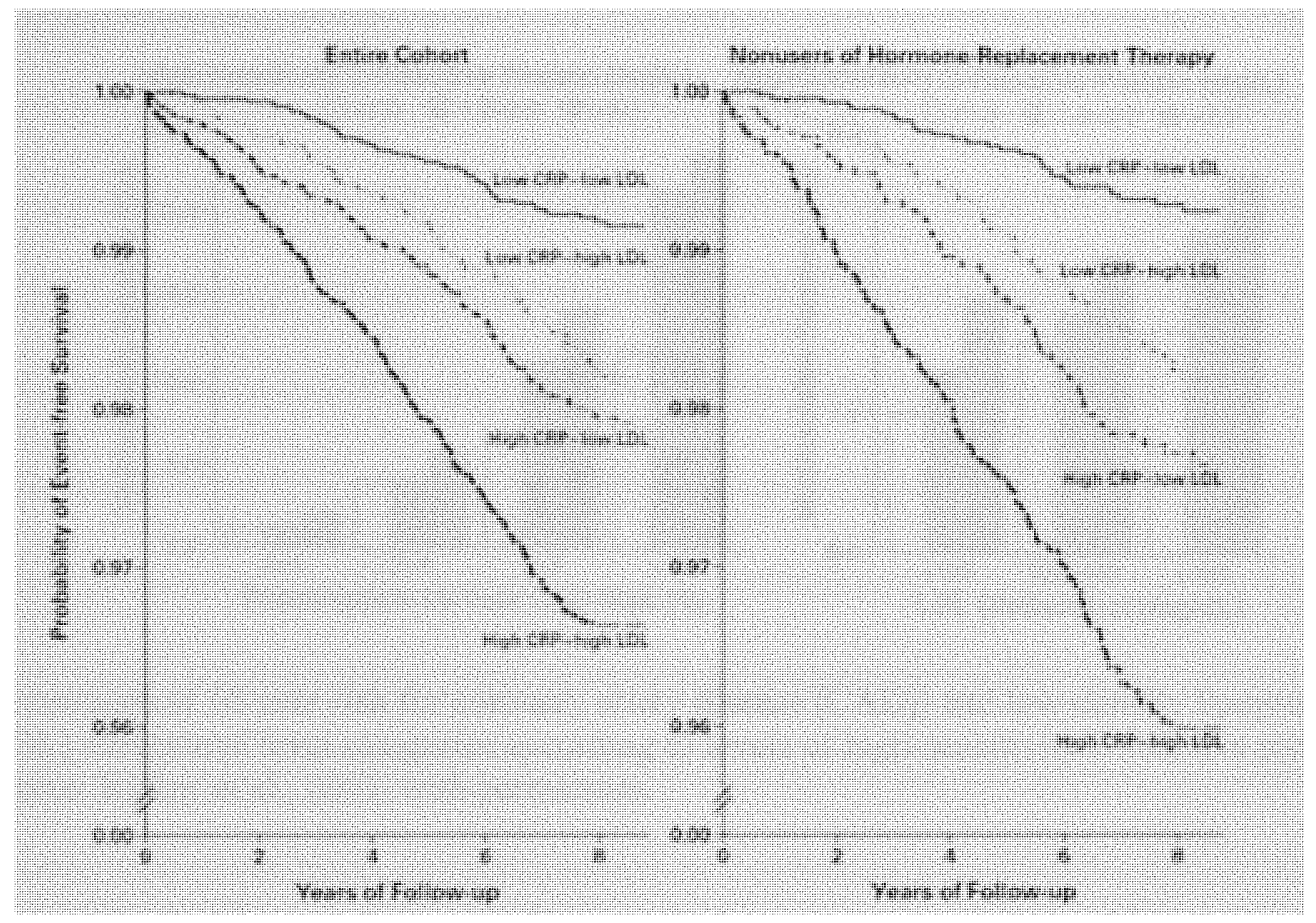

Data are shown for the entire cohort (27,939 women) and for women who were not taking hormone-replacement therapy at baseline ( 15,745 women). The median values were as follows: for hs-CRP, $1.52 \mathrm{mg} / \mathrm{L}$; for LDL-C, $123.7 \mathrm{mg} / \mathrm{dL}(3.20 \mathrm{mmol} / \mathrm{L})$. Note the expanded scale on the ordinate.

Source: Ridker et al., 2002, with permission. 
Figure 5 - Multivariable-adjusted relative risk of cardiovascular event by Framingham risk score

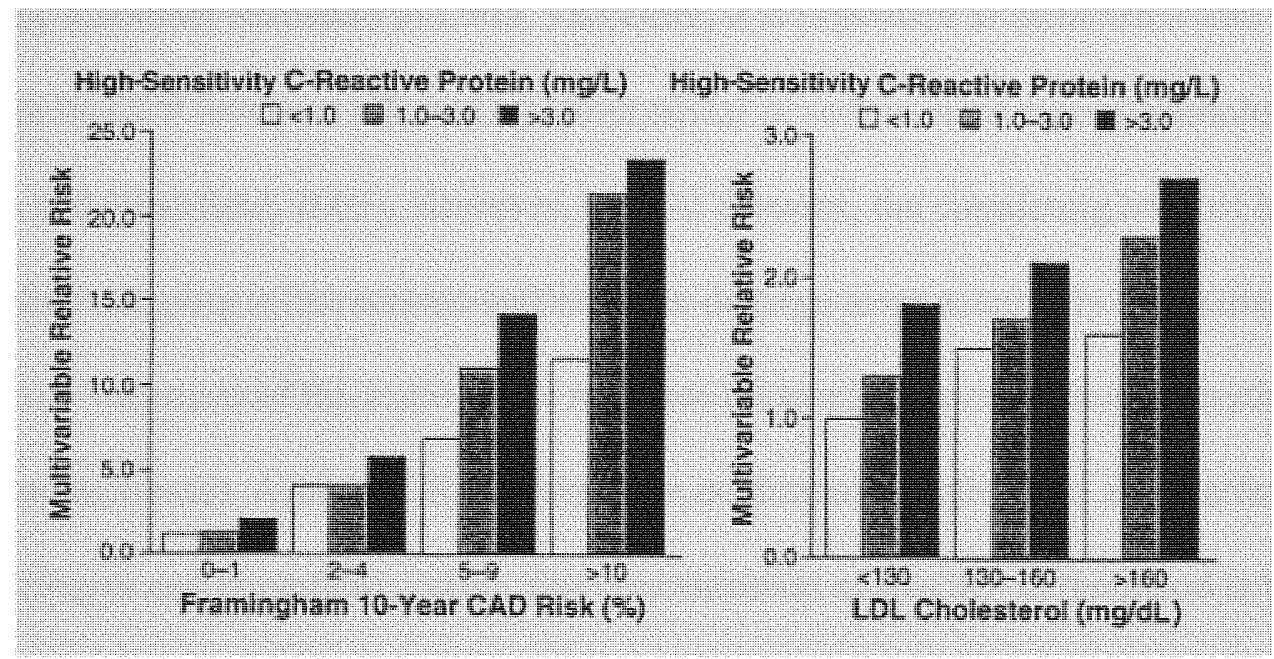

Percentages of 10-year risk for coronary artery disease [CAD]) and low-density lipoprotein (LDL) cholesterol level categories according to levels of high-sensitivity C-reactive protein. Source: Ridker et al., 2002, with permission.

Figure 6 - Cardiovascular event-free survival among apparently healthy individuals according to baseline hs-CRP levels.

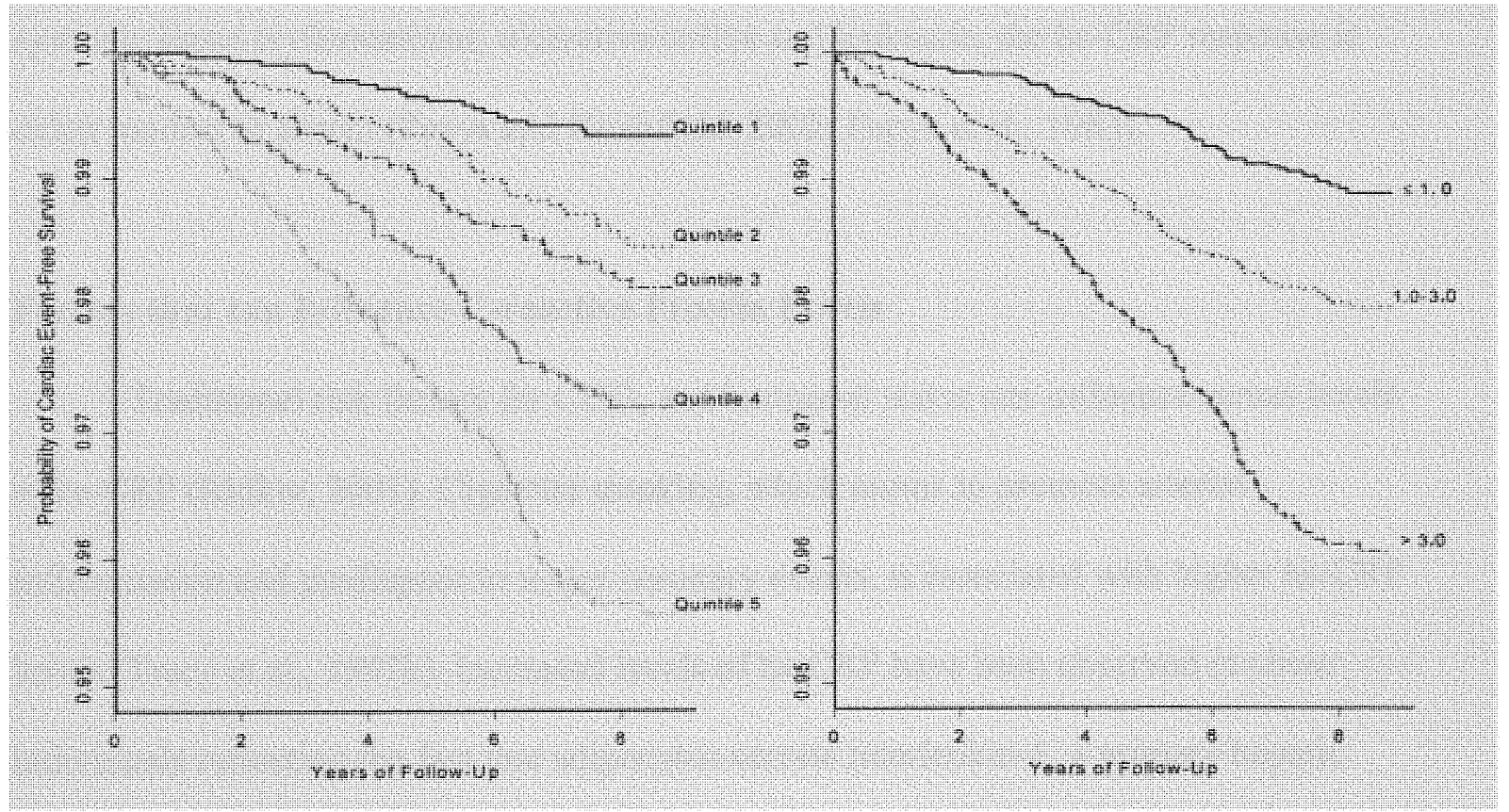

Data are shown using population-based quintiles for hs-CRP (left) and using 3 simple clinical cut-points for hs-CRP, $<1,1-3$, and $>3 \mathrm{mg} / \mathrm{L}$ (right) Source: Ridker, 2003, with permission. 
It is important to recognize that hs-CRP response is non-specific and is triggered by many disorders unrelated to CVD. Therefore, it is important to establish a true baseline hs-CRP level not affected by either trivial or serious inter-current pathologies (Pepys and Hirshfield, 2003). However, in most diseases, the circulating level of hs-CRP will reflect ongoing inflammation and/or tissue damage more accurately than other laboratory measures of the acute-phase response (example, plasma viscosity, erythrocyte sedimentation rate). Also, hs-CRP levels show no diurnal variation and are unaffected by eating (Pepys and Hirshfield, 2003). The concentration of hs-CRP in the blood is thus a very useful nonspecific biochemical marker of inflammation. The measurement of this marker will contribute importantly to (a) screening for organic disease, (b) monitoring of the response to treatment of inflammation and infection and, (c) detection of inter-current infection in immuno-compromised people. However, in a few specific cases, such as systemic lupus erythematosus, ulcerative colitis and leukemia, the hs-CRP response is either modest or absent (Pepys and Hirshfield, 2003; Pepys et al., 1983).

\section{Factors that can Influence C-reactive Protein Levels}

Traditional risk factors for $\mathrm{CHD}$, and hormonal status can influence serum levels of hs-CRP (Ridker, 2001). Cigarette smoking, which seems to promote arterial inflammation and predispose to major coronary events, is associated with higher hs-CRP levels (Tracy et al., 1997b). Increased levels of hs-CRP have been observed in obese individuals reflecting the fact that adipocytes secrete interleukin-6, a primary hepatic stimulant of hs-CRP (Visser et al., 1999; Yudkin et al., 1999) and, may also synthesize and secrete hs-CRP (Pepys And Hirschfield, 2003). There is evidence indicating that 
exercise reduces hs-CRP levels (Smith et al., 1999; Tisi et al., 1997). Individuals with diabetes (Ford, 1999) and the insulin resistance syndrome (Yudkin et al., 1999; Festa et al., 2000) have elevated serum hs-CRP suggesting that inflammation may play a role in these conditions. Similar results, showing hs-CRP levels to be associated with CVD risk have been demonstrated by Koenig et al (1999). They investigated and followed, for 8.2 years, 936 men between the ages of 45 to 64 years, who participated in the first Monitoring Trends and Determinants in Cardiovascular Disease (MONICA) Augsburg study, in Germany, between 1984 and 1985. They demonstrated that hs-CRP levels varied significantly with age, BMI, TC, smoking status, blood pressure and diabetes and, slightly with alcohol consumption, education, leisure-time physical activity and work activity.

Rhode et al (1999) have reported similar results for healthy men in the US. They investigated 1172 apparently healthy middle-aged (40-84 years) men who participated in the Physician Health Study. Significant positive associations were found between hs-CRP levels and age, number of cigarettes smoked each day, BMI, systolic and diastolic blood pressures, TC and TG. There were also significant inverse associations between hs-CRP and HDL-C, exercise frequency and apo A-1 and A-II. However, in multivariate analysis when age, smoking status, serum levels of tissue-type plasminogen activator antigen, fibrinogen, $\mathrm{Lp}(\mathrm{a})$ and total homocysteine and, several traditional risk factors for CHD were controlled, progressive increases in hs-CRP levels were still observed. These studies are suggesting significant association between hs-CRP levels and determinants of cardiovascular risks. However, these studies did not investigate hs-CRP levels and other 
determinants of CHD such as socio-economic status (SES), stress and knowledge of CHD.

There is limited data on hs-CRP levels in different populations. However, two large studies (Wener et al., 2000; Wong et al., 2001) using data from the NHANES III (1988-94) showed variation in hs-CRP levels by age, sex and ethnicity. In one of these studies (Wong et al., 2001) 4472 men and 5212 women 30-74 years old, without CHD, were evaluated. In this study, mean $( \pm \mathrm{SD})$ levels of hs-CRP in males $(0.41 \pm 0.64 \mathrm{mg} / \mathrm{dL})$ and females $(0.55 \pm 0.91 \mathrm{mg} / \mathrm{dL})$ were elevated. The mean hs-CRP levels in men were lower compared to values in women. This same study found that the hs-CRP levels in non-Hispanic Black men and Mexican-American women were higher than those in nonHispanic Whites. There is no published data for foreign-born and US-born Afro Caribbean Americans.

\section{Total Cholesterol/High-density Lipoprotein Cholesterol Ratio}

Current guidelines recommend using LDL-C levels and other risk factors to identify individuals at risk of CHD (NCEP: ATP III, 2001). However, there are studies indicating that almost half of all individuals with CHD have normal LDL-C but elevated CRP levels (Rifai and Ridker, 2001) and low HDL-C with or without elevated triglyceride levels (Goldbourt et al., 1997; Rubins et al., 1995; Natarajan et al., 2003). There is evidence indicating that the total cholesterol/HDL-Cholesterol ratio (TC/HDLC) is a powerful predictor for CHD risk (Kinosian et al., 1995; Criqui and Golomb, 1998). The ratio reflects two powerful components of risk: (1) high total cholesterol is a marker for atherogenic lipoproteins and (2) low HDL-Cholesterol correlates with the 
multiple risk factors of the metabolic syndrome and may impart some independent risk (NCEP: ATP III, 2001). Low-density lipoprotein cholesterol is now recognized as the atherogenic component of total cholesterol levels, whereas HDL-C is inversely related to the atherogenic process (Kannel et al., 2004). It is suggested that the TC/HDL-C ratio may be a better predictor of the dyslipidemic atherosclerotic process at all ages (Natarajan et al., 2003; Kannel et al., 2004). There is evidence indicating that changes in the TC/HDL-C ratio may be superior at predicting individuals who will or will not develop CHD compared to changes in LDL-C alone (Konosian et al., 1995; Grover et al., 1995). Konosian et al (1995) and Grover et al (1995), using data from the Framingham Heart Study and the Lipid Research Clinics Population Prevalence Study, have shown that the TC/HDL-C ratio is a better predictor of CHD risk, even when compared with newer risk factors, such as $\mathrm{Lp}(\mathrm{a})$, homocysteine, fibrinogen and tissue plasminogen activator antigen (see also Figure 3 , page 68). It is suggested that the readily available measures such as TC/HDL-C ratio should be incorporated in the current NCEP: ATP III Guidelines, both for the identification of individuals at very high risk for CHD and for monitoring cholesterol-lowering therapy (Natarajan et al., 2003). A TC/HDL-C ratio of $\leq$ 3.5 (males and females) is associated with a lower risk for CHD whereas TC/HDL-C ratios of $\geq 5.0$ are associated with higher risk for CHD (Sonnenberg et al., 1996; Kannel, 1983). There is no published data for foreign-born and US-born Afro Caribbean Americans. 


\section{Improving the Health of Caribbean Americans}

There is evidence showing lower all-cause mortality due to CHD in foreign-born Blacks compared to US-born Blacks and US-born Whites (Fang et al., 1997; Singh and Siahpush, 2002). In these studies, Blacks included both non-Hispanic Blacks and Hispanic Blacks. Fang et al (1996) also, showed that Caribbean Americans in New York City had substantially lower all-cause and cardiovascular mortality rates compared to Blacks born in the southern and northeastern USA. However, risk factors for CHD were not investigated in this study.

Most of the studies investigating risk factors for CHD (including CRP levels) have been done with adults aged between 40 and 84 years. There is a dearth of information on CHD risk factors (such as cigarette smoking, blood pressure values, physical activity, blood glucose and lipids levels) in foreign-born and US-born Caribbean Americans. Also, the influence of diet, body weight, perceived stress, knowledge of CHD and CRP levels on risks for CHD is unknown in this population.

Ethnic populations' beliefs and practices can influence the interactions between healthcare provider and individuals of this population. However, insufficient attention is paid to the area of ethno-medicine (Bronner, 1994). Information about an individual ethnic group is important for the identification, tracking and investigation of the reasons for ethnic differences in the prevalence and severity of disease and in response to treatment (Burchard et al., 2003). Also, information about a person's ethnic group is important in identifying different risk factor profiles even when a disease does not occur with dramatically different frequencies in the different ethnic groups (Burchard et al., 2003). It is suggested that by focusing on the ethnic and cultural differences in a 
population we can better understand the variations among different ethnic and cultural groups and the influence of migration on the prevalence and severity of diseases (Burchard et al., 2003). This focus can provide the opportunity for healthcare professionals and policy planners to develop appropriate programs and strategies for the improvement of health outcomes.

No study has been done to evaluate lifestyle and biochemical risk factors for CHD in foreign-born and US-born Caribbean Americans, especially in South Florida, where there is a growing population. Foreign-born Caribbean Americans may have different health perceptions, beliefs, behaviors, dietary habits and propensities to report health/nutritional conditions compared to US-born Caribbean Americans and African Americans. Also, there may be differences in socio-economic status, exposure to knowledge about CHD, physical activity and stress. These differences may be distinguishing features of these groups and may influence risk factors for cardiovascular disease in this population of African ancestry (Curtis and Lawson, 2000).

Designing effective prevention programs to reduce the risk of CHD requires responding to the needs of different ethnic groups (Dundas et al., 2001). This research determined and compared behavioral/environmental, bio-physiological and emerging determinants of CHD in apparently healthy Afro Caribbean American (foreign- and USborn) and African American adults (18-40 years old) in South Florida. Data from this study may also prove useful to healthcare professionals with little knowledge of or, familiarity with Afro Caribbean cultures. 


\section{CHAPTER III \\ METHODOLOGY}

\section{Study Design}

A cross sectional survey study design (Newman et al., 2001) using a convenience sample of individuals 18 to 40 years old from three groups (Foreign-born Afro Caribbeans, first generation Afro Caribbean Americans [US-born] and African Americans) was employed. Participants were surveyed only once.

In the published literature, Blacks and/or African Americans are terms used interchangeably to describe all persons of African ancestry. African Americans and Afro Caribbeans are participants from the same race but different ethnic and cultural backgrounds. For the purpose of this study the ethnic groups were defined as follows:

(a) Foreign-born Afro Caribbean Americans (FBCA) were individuals of African ancestry (non-Hispanic) born in one of the Caribbean Islands (i.e. Jamaica, Haiti, Trinidad and Tobago, Barbados or other small Caribbean Islands) and residing in the US for less than 10 years (Fang et al., 1996 showed mortality differentials due to CVD between foreign-born [13.9 years being the average length of stay in the US] and US-born Blacks). Haitians were included in the study if they understood and spook English.

(b) United States-born (first and subsequent generations) Afro Caribbean Americans (USBCA) were individuals born in the US to both Caribbean-born parents. 

the US to both parents who are also born in the US. The place of birth of participants and their parents was requested at the initial screening interview.

\section{Study Population}

The study participants were recruited from the Miami-Dade and Broward counties in South Florida. Individuals from the Caribbean, Caribbean American and African American populations were recruited. There is $85 \%$ power in a $2 \times 3$ analysis of variance (ANOVA) to detect a medium sized $(d=0.25)$ main effect or interaction with a 0.05 significance level (Cohen, 1988) using sample sizes of 60 respondents for each of the three ethnic groups. Based on the previous assumptions, the total sample size was 180 individuals (30 males, 30 females) from each of the three ethnic groups: In this study, risks of coronary heart disease (CHD) for the age range 18-40 years were considered low (NCEP: ATP III, 2001). Also, risks of CHD for both gender groups were assumed to be similar (NCEP: ATP III, 2001) and, thus, equal numbers of males and females were recruited. However, the actual number of subjects recruited for the study is shown in Table 7. The study was approximately 12 months in duration. 
Table 7 - Actual Sample Size for the Study Population

\begin{tabular}{|c|c|c|c|c|}
\hline & \multicolumn{3}{|c|}{ ETHNIC GROUPS } & \\
\hline GENDER & $\begin{array}{l}\text { Foreign-born } \\
\text { Caribbeans } \\
\text { (FBCA) }\end{array}$ & $\begin{array}{l}\text { US-born Caribbean } \\
\text { Americans } \\
\text { (USBCA) }\end{array}$ & $\begin{array}{l}\text { African } \\
\text { Americans } \\
\text { (AA) }\end{array}$ & Total \\
\hline Males & 31 & 30 & 30 & 91 \\
\hline Females & 35 & 32 & 31 & 98 \\
\hline Total & 66 & 62 & 61 & 189 \\
\hline
\end{tabular}

\section{Inclusion Criteria}

Participants were included in the study if they met the following criteria:

(a) Were from the targeted groups (FBCA \& living in the US $<10$ years, USBCA; both parents Afro Caribbean ancestry, AA; both parents AA ancestry).

(b) Between the ages of 18 and 40 years.

(c) Willing to sign an informed consent form.

(d) Not regularly taking anti-inflammatory drugs (aspirin Motrin, Advil)

\section{Exclusion Criteria}

(a) Not from the defined ethnic groups.

(b) Less than 18 years and older than 40 years.

(b) Diagnosed with any of the following medical conditions: cancer, cardiomyopathy, 
gout, migraine, peptic ulcer, psychiatric, renal, thyroid and hepatic diseases (selfreported).

(c) Taking over the counter herbal or prescription medications (anti-inflammatory agents, Anti-asthmatics, anti-depressants, anti-convulsives).

(d) Pregnant

(e) Chronic alcohol users (three or more drinks per day or per sitting). One drink was defined as: $12 \mathrm{oz}$. of $4.5 \%$ beer; 4 to $5 \mathrm{oz}$ of $14 \%$ wine; $10 \mathrm{oz}$. wine cooler; 1 to $11 / 2$ oz. distilled liquor ( $80 \%$ proof whiskey, scotch, rum, vodka).

\section{Pilot Study}

Initially, a convenience sample (different from the main population) of 10-15 Afro Caribbeans/Afro Americans from the Florida International University community was recruited, for validation of the research tools (Block food frequency, Baecke physical activity, Cohen perceived stress, knowledge of CHD risk factors questionnaires) that were used in this study. Each participant completed a 24-hour dietary recall at the same time the Block FFQ was given. For the test-retest reliability of the Baecke physical activity, Cohen perceived stress and the "knowledge of CHD risk factors" questionnaires, each participant completed these questionnaires a second time when they returned the first set of the questionnaires to the investigator. The participants were also asked to complete an activity log that asked questions about type of activity during the past month. The data were analyzed (information given in the results section) and the tools adjusted, if necessary. 


\section{Recruitment of Subjects}

Recruitment was based on the following methods: 1) Flyers (Appendix A) with the study outline and the inclusion criteria, benefits, the investigator's telephone numbers and e-mail address, were distributed and placed on notice boards at the Florida International University (FIU) and on notice boards at strategic locations in Miami-Dade and Broward counties. We also met with Caribbean American and African American Student Associations at FIU, distributed flyers and discussed the purpose of the study.

Subjects who were interested in the study were asked to contact the investigator by telephone or electronic mail (e-mail). During the initial telephone interview/e-mail contact, the investigator explained the purpose, procedures, risks and benefits of the study, the approximate length of time involved, determined eligibility and willingness to sign an informed consent form. Individuals who were interested in the study were asked to report on a specified day, in an overnight fasting state, to the Human Nutrition Research Laboratory (Health and Life Sciences Building, [HLS] 430), at the College of Health and Urban Affairs (CHUA) at FIU. When the participants visited our laboratory they were given an opportunity to review the study materials, ask questions and decide if they wanted to participate in the study. Those who decided to participate in the study, after meeting the inclusion criteria, were asked to sign an Informed Consent (IC) form (Appendix B) approved by the Institutional Review Board (IRB) at FIU (approval \# 010803). After the IC form was signed, each participant's blood pressure (taken twice), weight and height measurements were taken. They were asked to give one tablespoon of blood and given a packet that included the Socio-demographic Questionnaire (Appendix C), the Cohen Perceived Stress Questionnaire (Appendix D), the Knowledge of CHD 
Questionnaire (Appendix E), the Food Frequency Questionnaire (Appendix F) and the Baecke physical activity questionnaire (Appendix G). Only one visit was necessary to collect all of the required data. The entire process took approximately 60 minutes.

\section{DATA COLLECTION}

The following data sets were collected from each subject:

\section{Socio-demographic Profile}

a Socio-economic data (age, country of origin, education attainment and household income, family history of CHD, medical insurance coverage, healthcare visits)

a Cohen Perceived Stress Instrument

Knowledge of CHD Risk Factor Instrument

\section{Behavioral/environmental Assessment}

․ Dietary - Block Food Frequency Questionnaire (included information on cigarette smoking and alcohol consumption)

- Baecke Physical Activity Assessment Instrument

口 Anthropometry - Body Weight and Height

\section{Physiological Assessment}

a Blood Pressure

․ Fasting Blood Glucose

a Fasting Total Cholesterol (TC), Low-density Cholesterol (LDL-C), Triglyceride (TG), High-density Lipoprotein Cholesterol (HDL-C)

\section{Emerging Risk Factors Assessment}

a High-sensitivity C-reactive Protein (hs-CRP), TC/HDL-C ratio 


\section{Socio-Demographic Questionnaire (SDQ) (Appendix C)}

The Socio-demographic questionnaire (SDQ) included information on age, gender, ethnic background, education, household income, employment status, marital status, health insurance, place of birth, frequency of healthcare contact, number of individuals in the household, personal and family history of CHD, diabetes and length of residency in the US.

Socio-economic Data: As part of the SDQ, educational level attained and household income were used to assess SES. Educational attainment was estimated by each participant indicating on the SDQ the highest year of school completed. Educational attainment was categorized into those with less than or equal to high school education, those with college education but without college degree and, those with a college degree. Participants indicated on the demographic questionnaire the annual household income bracket in which they belong. The respondents were classified into three income groups $(<\$ 20,000, \$ 20,000-\$ 39,999, \geq \$ 40,000$ per year $)$. An indication of per capita income was calculated by dividing household income by the number of individuals in the household. In 2002, a family of five earning $<\$ 22,377$ per year, a family of four earning $<\$ 18,556$ per year, a family of three earning $<\$ 14,072$ per year, a family of two earning $<\$ 12,047$ and an individual earning less than $\$ 9,359$ were considered below the poverty line (US Census Bureau, 2002: Poverty). 


\section{Perceived Stress Assessment (Appendix D)}

An evaluation of perceived stress level was done using a self-administered 14item Perceived Stress Scale (PSS) (Cohen et al., 1983; Cohen and Williamson, 1988) (Appendix D). The PSS can be administered in a few minutes and, is a better predictor of health outcomes than a global measure of objective stressors such as life-event scales (Cohen et al., 1983). It is more sensitive to chronic stress deriving from ongoing life circumstances, to stress from expectations concerning future events, to stress from events not listed on a particular life-event scale, and to reactions to the specific events included on any scale (Cohen et al., 1983). Time reference for PSS measure is the past month. The areas covered refer to feeling in control, feeling nervous or stress, confidence in handling personal problems, life hassles and feeling irritated, angry and, upset. A five-point Likert scale (never, almost never, sometimes, fairly often, very often) was used to assess stress level. The PSS was obtained by reversing the scores $(0=4 ; 1=3 ; 2=2$; etc $)$ on the seven positive items (items $4,5,6,7,9,10,13$ ) and then summing across all 14 items. A single score was generated which represented overall level of stress. One half of the items was scored positively (i.e. greater stress) and the other half, reverse-scored because they were phrased to indicate lower stress. The items were general in nature, relatively free of content specific to any population group and thus, applicable to any population.

\section{Knowledge of CHD Risk Factors Questionnaire (Appendix E)}

Assessment of participants' knowledge of CHD risk factors was measured using the "Check Your Healthy Heart I.Q." (NIH Publication No. 93-2724) and "Check Your Cholesterol and Heart Disease I.Q." (NIH Publication No. 95-3794) questionnaires, 
developed by the National Heart, Lung and Blood Institute (NHLBI). The "Check Your Healthy Heart I.Q." (Questions 1-14) and "Check Your Cholesterol and Heart Disease I.Q." (Questions 15-25) questionnaires contain 14 and 11 "True" and "False" questions, respectively (Appendix E). Participants received a copy of the right answers and an explanation after completing the questionnaire. Participants' knowledge of risk factors for CHD (questions 1-14) and, knowledge and awareness of cholesterol and fat (questions 15-25) were analyzed and the scores added for analysis. The items are general in nature, relatively free of content specific to any population group and thus, applicable to any population.

\section{Dietary Assessment (Appendix F)}

\section{Food Frequency Questionnaire (FFQ)}

Dietary information was collected using a validated FFQ (Appendix F) (Block et al., 1986; Block et al., 1990; Subar et al., 2001). The scannable version of this questionnaire was used. Included with this questionnaire were pictorial representations of portion sizes to help the participants with portion size selections. Participants were asked to indicate on a separate form any food they had eaten during the past year that was not listed on the Block FFQ. The Block FFQ has been substantially revised, based on the National Health and Nutrition Examination Survey III (NHANES III) food intake data. This revised FFQ includes foods representing at least 90 percent of the intakes of each of the nutrients on the NHANES III database (National Center for Health Statistics, DHHS Publication, 94-1308). The food list was derived separately for African Americans (inclusive of foreign and US-born Afro Caribbeans), Whites and Hispanics, to ensure that 
major nutrient contributors for each of those groups were included. Nutrient content database was developed using NHANES III and CSFII survey databases and the United States Department of Agriculture (USDA) Standard Reference database (www.nal.usda.gov). The questionnaire consisted of 109 food items, as well as additional questions to assess use of fat-modified foods, types of milk used, servings of fruits and vegetables, frequency and type of fat used in cooking, and other nutrient-related behaviors. We also asked each participant to report any additional foods (local or international) eaten that were not stated on the FFQ. This FFQ also contained questions about drinking and cigarette smoking habits. The intakes of selected vitamins, minerals and supplements such as vitamins $\mathrm{A}, \beta$-carotene, $\mathrm{C}, \mathrm{E}, \mathrm{B}_{6}, \mathrm{~B}_{12}$, folate/folic acid, selenium, calcium, sodium, iron, zinc and fish oil were assessed. Food models, measuring utensils, ruler and imperial to metric conversion materials were used to help estimate portion sizes. Nutrients of interest included those recommended by the American Heart Association (AHA Statistical Update, 2002) to be important in a heart-healthy diet and including those that may influence CHD (Willett, 1998). These were: Energy (kilocalorie, kcal); Protein (grams, g); percent (\%) kcal from protein; Carbohydrate (g); percent kcal from carbohydrate; Dietary Fat (g) (saturated, monounsaturated, polyunsaturated, omega$3(\omega-3)$ fatty acid, (mg)); percent kcal from fat; Cholesterol (mg); Dietary Fiber (g) (soluble and insoluble); selected minerals (Sodium, [mg], Potassium, [mg], Calcium, [mg], Iron, [mg], Selenium, [mg], Copper, [mg],); vitamins (C, [mg], E, [mg alphatocopherol Equivalent], $B_{6},[\mathrm{mg}], B_{12},[\mathrm{mg}]$, folate, $[\mu \mathrm{g}]$, servings of fruits, vegetables and legumes, and frequency and type of fat used in cooking. 
A diet score was devised according to whether the participants met recommended dietary requirements for 7 selected nutrients (percent energy from fat, saturated fat, dietary cholesterol, fiber, sodium, fruit and vegetable servings) or not. Individuals who exceeded the dietary recommendations for percent energy from fat, saturated fat, cholesterol and sodium were assigned a score of 0 and a score of 1 if they met the dietary requirements. Participants who consumed below the dietary recommendations for dietary fiber, fruit and vegetable servings were assigned a score of 0 and 1 if they met the dietary requirements. This score was summed across the selected food items to give a total diet score. The diet score ranged from $0-7$. Higher scores were indicative of better quality diets.

Food frequency questionnaires (FFQs) are commonly used in epidemiological studies of chronic disease to evaluate usual and long-term dietary intakes of individuals. Accurate information about food intake is important because they may be independently associated with disease outcomes. Compared to diet histories and 24-hour recalls, FFQs require neither specialized training nor lengthy interviews. Thus, respondent's burden is light, with subsequent increased participation. The administration of FFQs can be done by telephone, mail, interviewer to interviewee (face to face) or be self-administered. This will lead to lower costs, especially in large-scale studies. There is evidence indicating that the FFQ has adequate test-retest reliability and that measures of reliability were comparable to those of food records (Willett et al., 1985). 


\section{Dietary Analysis}

The FFQ was sent to the Block Dietary Data Systems (Berkeley, CA) for dietary analysis. The FFQ data was coded, entered and analyzed by using techniques developed by Block et al., (1986). We also used the Caribbean Food and Nutrition Institute's (CFNI) Food composition database (Caribbean Food and Nutrition Institute, 1995; 2000) to aid in the assessment of the food intake of Caribbean Nationals. This database is a compilation of foods from Britain, the Caribbean and the US.

\section{Physical Activity (Appendix G)}

Physical activity was assessed using the validated Baecke physical activity questionnaire (validated also for blacks or African Americans, inclusive of foreign and US-born Afro Caribbeans) (Baecke et al., 1982; Kriska and Caspersen, 1997; Atherosclerosis Risk in Communities Study, 1989). This tool has been validated against other methods of assessing physical activity (Kriska and Caspersen, 1997) and in studies assessing CVD risk factors (Rodriquiz et al., 1994; NIH, 1995; Leon et al., 1997; McMurray et al., 1998). This questionnaire (16 questions) assesses leisure and occupational activities. It consists of three sections: work activity (questions 1-8), sports activity (question 9-12) and non-sports leisure activity (questions 13-16). Each section is scored on a five-point Likert scale, ranging from never to always or very often. For two of the most frequently reported sports activities, additional questions query the number of months per year and hours per week of participation. The three sections are further divided into three levels: 
Occupational physical activity: low level for occupations such as clerical work, driving, shop-keeping, teaching, studying, housework, medical practice and all other occupations with a university education; middle level for occupations such as factory work, plumbing, carpentry, and farming; and high level for occupations such as dock work, construction work and sport.

Sports: low level for sports such as billiards, sailing, bowling and golf (average energy expenditure (AEE) $0.76 \mathrm{MJ} / \mathrm{h}$ ); middle level for sports such as badminton, cycling, dancing, swimming and tennis (AEE $1.26 \mathrm{MJ} / \mathrm{h}$ ); and high level for sports such as boxing, basketball, football, soccer, rugby and rowing (AEE $1.76 \mathrm{MJ} / \mathrm{h}$ ). The sport score was calculated from a combination of the intensity of the sport which was played, the amount of time spent per week playing that sport and the amount of time during the year that the sport was played.

The total scores for each of the work, sports and non-sports leisure time activities were added to give the total score. The scores were then classified into quartiles for general classification of activity levels. There is evidence that self-assessment of physical assessment activity parallels the scores of actual physical activity reasonable well (Chave et al., 1978).

Anthropometry: As part of the SDQ, anthropometric data such as weight (collected as pounds, [lb], to the nearest half $\mathrm{lb}$, and expressed as kilograms, $[\mathrm{kg}]$ ), and height, (collected as inches, [in], to the nearest half in, and expressed as meters, [m], on a beam balance (calibrated weekly with a reference weight) were collected. These measurements were taken with the participants in light clothing and in socks. Waist circumference 
(collected as inches, [in] to the nearest half in, and (centimeter, $\mathrm{cm}$ ) was measured with each subject standing erect, abdomen relaxed, arms at their sides and feet together. The measurement was taken using a nonstretchable tape measure at the end of a normal expiration, without the tape compressing the skin. The tape is placed around the subject, in a horizontal plane, at the level of the natural waist, which is the narrowest part of the torso, as seen from the anterior aspect (Callaway et al., 1988). Body Mass Index (BMI), was calculated as weight (kilograms, $\mathrm{kg}$ ) divided by height squared (meters, $\mathrm{m}^{2}$ ). The BMI is highly correlated with body fat than any other indicator of height and weight and, was used to assess body fat (overall adiposity). Individuals with BMI less than 18.5 were considered underweight, between 18.5 and 24.9 normal, between 25.0 and 29.9 overweight and over 30.0 as obese. Waist circumference is positively correlated with abdominal fat content. Individuals with BMI 25.0 to 34.9 and waist circumference $>102$ $\mathrm{cm}(>40 \mathrm{in})$ males and $88 \mathrm{~cm}$ ( $>35 \mathrm{in})$ in females are at high to very high risk of developing obesity-related risk factors for CHD.

\section{Blood Pressure}

The blood pressure measurements of the participants were taken in the sitting position in a quiet, comfortable room following a 10 minutes of rest, by a trained assistant using a random zero sphygmomanometer (Tycos 5090-02 Welch Allyn Pocket Aneroid Sphygmomanometer, Arden, NC) and a stethoscope (Littmann Cardiology. 3M, St. Paul, MN). The appropriate adult-size arm cuff was used in order to record accurate measures. The appropriate blood pressure cuff was placed approximately one inch above the antecubital space of the right arm (a natural crease of the elbow), with the center of 
the bladder applied directly over the brachial artery in the antecubital space. The cuff size was determined by the width of the inflatable bladder, which was about $40 \%$ of the circumference from the midpoint of the arm. The measurements were read with the forearm and the manometer at the level of the heart. The cuff was inflated by squeezing the rubber bulb. The pressure was released at a rate of $2 \mathrm{~mm} \mathrm{Hg}$ per second after the monometer indicator exceeded $180 \mathrm{~mm} \mathrm{Hg}$. As the pressure fell, systolic blood pressure was determined at the point where the initial pulse sound was heard; Korotkoff's fifth phase was used to determine diastolic blood pressure. The cuff was deflated completly at the end of the measurement.

The first blood pressure reading was taken at the beginning of the study, with the subject seated after 10 minutes of rest. A total of two blood pressure readings were taken from each participant at least 10 minutes apart and the average calculated and recorded. Blood pressure was considered normal if the mean of two measurements was $\leq 120 / 80$ mm Hg; prehypertension if 120-139/80-89 mm Hg; stage 1 hypertension if 140-159/90$99 \mathrm{~mm} \mathrm{Hg}$ and stage 2 hypertension if $\geq 160 / 100 \mathrm{~mm} \mathrm{Hg}$ (JNC VII, 2003).

\section{Biochemical Data Collection (Blood Glucose, Lipids, hs-CRP)}

Fasting blood was collected by a licensed Phlebotomist, using standard procedures. Blood was collected from each participant after the IC form was signed, at the first visit to the Human Nutrition Laboratory at the CHUA at FIU. After the blood was coagulated (30 minutes), it was centrifuged at full speed (1100 RPM) for 15 minutes and the serum removed, placed in labeled plastic tubes for the determination of serum lipids (total cholesterol (TC), high-density lipoprotein cholesterol (HDL-C) and 
triglycerides, TG) by the methods of McNamara and Schaefer (1987) using the Beckman Synchron CX System (Global Chemical, INC., US). Very low-density lipoprotein cholesterol (VLDL-C) was estimated as TG/5. Low-density lipoprotein cholesterol (LDLC) was calculated by the Friedewald formula (Friedewald et al., 1972): LDL-C $=$ TC (TG/5 + HDL-C). TC values $\geq 200 \mathrm{mg} / \mathrm{dL}, \mathrm{LDL}-\mathrm{C}$ values $\geq 130 \mathrm{mg} / \mathrm{dL}$ and TG values $\geq$ $200 \mathrm{mg} / \mathrm{dL}$ were considered elevated whereas, $\mathrm{HDL}-\mathrm{C}<40 \mathrm{mg} / \mathrm{dL}$ was considered undesirable (NCEP: ATP III, 2001).

Fasting blood glucose was analyzed by enzymatic procedures using the automatic Chemistry Analyzer (Global Chemical, INC., US). Fasting blood glucose was considered normal if $<110 \mathrm{mg} / \mathrm{dL}$, glucose intolerant if between $110-126 \mathrm{mg} / \mathrm{dL}$ and elevated if $>$ $126 \mathrm{mg} / \mathrm{dL}$.

Serum high-sensitivity CRP (hsCRP) levels were assayed using the Food and Drug Administration approved latex-enhanced nephelomtric assays (Rifai et al., 1999) on a BN II analyzer (Dade Behring, USA). Levels of hs-CRP $>3 \mathrm{mg} / \mathrm{L}$ will be considered elevated (Ridker, 2003; Pearson et al., 2003).

\section{Statistical Analysis}

The following statistical analyses were done to address the purpose, specific objectives and research questions of this study. Validation of the Block FFQ for this population was evaluated by comparing dietary analyses of the Block FFQ and the 24hour dietary recall. Test-retest validation of the Baecke Physical Activity, the Cohen Perceived Stress and the "Knowledge of CHD Risk Factors" questionnaires was evaluated by comparing the "test" and "retest" scores of these questionnaires from the 
pilot study. Summary statistics using quantitative data from the SDQ, PSS, "Knowledge of CHD Risk Factors", FFQ, Baecke physical activity questionnaires and, information from the measured parameters such as weight/height (evaluated as body mass index), waist circumference, blood pressure, blood glucose, blood lipids, hs-CRP and, categorical variables from the socio-demographic questionnaire, such as ethnicity, place of birth, occupation, employment status, education status and household income were calculated using SPSS for Windows (v. 12.0). The results (socio-demographic, behavioral/environmental, physiological and emerging determinants of CHD) were expressed as mean \pm standard deviation, frequencies and percentages and compared by gender, ethnic groups and gender-ethnic subgroups (Research Questions 1 - 4). One-way analysis of Variance (ANOVA) and chi-square analysis were performed as appropriate. Pair-wise comparisons using Bonferroni's posthoc tests were performed to adjust for multiple comparisons (FBCA vs USBCA, FBCA vs AA, USBCA vs AA). Pearson's correlations were also performed to evaluate associations between the sociodemographic, behavioral/environmental, physiological and emerging risk factors for CHD. Differences were considered significant at $\mathrm{p}<0.05$. 


\section{CHAPTER IV}

\section{RESULTS}

This study determined and compared socio-demographic, behavioral/environmental, bio-physiological and emerging risk factors for coronary heart disease (CHD) in apparently healthy foreign-born Afro Caribbean (FBCA - residence in the US $<10$ years), US-born Afro Caribbean American (USBCA) and African American (AA) adults aged 18-40 years in South Florida.

The results of this study are organized according to the research questions and are presented in five sections. Section 1 covers the participants' socio-demographic information. Sections 2 to 4 cover the participants' behavioral/environmental, physiological and emerging risk factors for CHD. Reports on the correlations between the socio-demographics, behavioral/environmental, bio-physiological and emerging risk factors for $\mathrm{CHD}$ are presented in Section 5. The high test/retest correlations of the Baecke physical activity $(r=0.933 ; p<0.001)$, Cohen perceived stress $(r=0.986 ; p<0.001)$ and "knowledge of CHD risk factor" ( $r=0.927 ; \mathrm{p}<0.001$ ) questionnaires (Appendices D, E and $G$ ) indicated that the questionnaires were very reliable for this study population.

\section{Section 1 - Research Question 1: Are the socio-demographic profiles of FBCA, USBCA and AA adults similar?}

Over 400 individuals were contacted and/or responded to posted flyers, FIU-wide email advertisements, classroom presentations and presentations made at churches and school-based Afro Caribbean/African American Student Organizations. However, only 
those who met the inclusion criteria $(\mathrm{N}=189)$ of the research protocol and signed the IC form participated in the study.

\section{Country of Birth}

The majority of male $(n=25,80.6 \%)$ and female $(n=27,77.2 \%)$ FBCA participants were from Jamaica, Haiti, Trinidad and Tobago (Figure 7). The other 19.4\% of the male and $22.8 \%$ of the female FBCA were from the Dominican Republic, Antigua, the Cayman Islands, Dominica, Guyana and St. Lucia. The length of residence in the US of the male and female FBCA participants, ranged from 1 to 10 years. No significant difference was seen between the FBCA male and female participants residing in the US for $1-3(32.3 \%$ vs. $28.6 \%), 4-5(19.4 \%$ vs. $28.6 \%)$ or $6-10$ years $(45.2 \%$ vs. $40.0 \%)$. The majority of male and female USBCA participants were from Florida $(n=20,66.7 \% ; n=22$, $68.8 \%)$ and New York $(n=5,16.7 \% ; n=5,15.6 \%)$, respectively. The other $16.6 \%$ males and $15.6 \%$ females USBCA came from California, Georgia, Massachusetts, New Jersey and North Carolina (Figure 7). The male and female AA participants were mainly from Florida $(n=22,73.3 \% ; n=16,51.6 \%)$ and New York $(n=4,13.3 \% ; n=4,12.9 \%)$, respectively. The other $13.3 \%$ of the male and $35.5 \%$ of the female AA were from Chicago, Georgia, Maryland, Tennessee, Virginia and Washington, DC (Figure 7). The parents of the male and female FBCA participants were mainly from Jamaica ( $n=16$, $51.6 \% ; n=16,45.7 \%)$, Haiti $(n=5,16.1 \% ; n=7,20.0 \%)$ and Trinidad and Tobago $(n=4$, $12.9 \% ; n=5,14.3 \%$ ), respectively (Figure 8). Most of the parents of the males and females USBCA participants were born in Jamaica $(n=17,56.7 \% ; n=11,34.4 \%)$ and Haiti $(n=10,33.3 \% ; n=15,46.9 \%$ ) (Figure 8). 


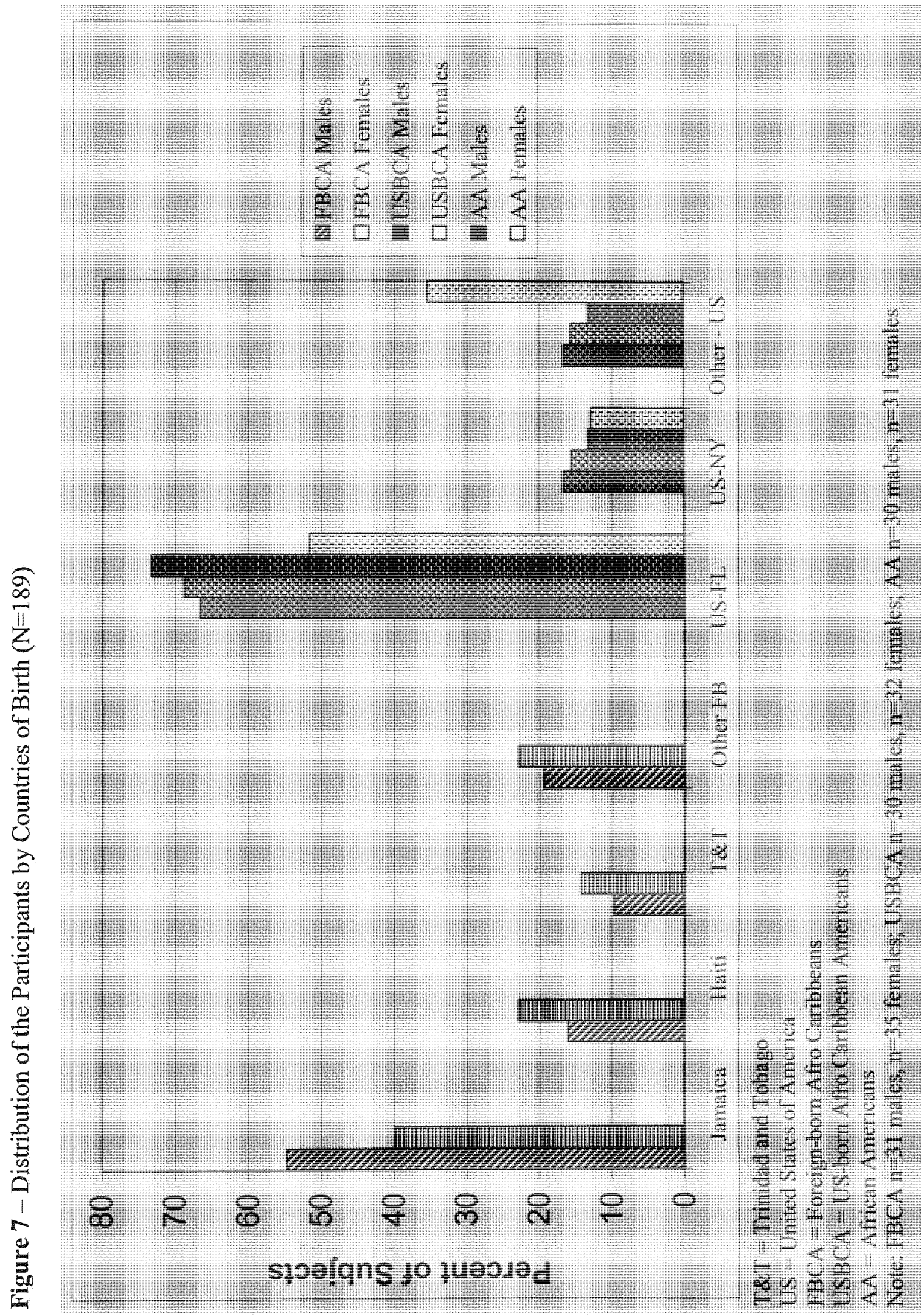




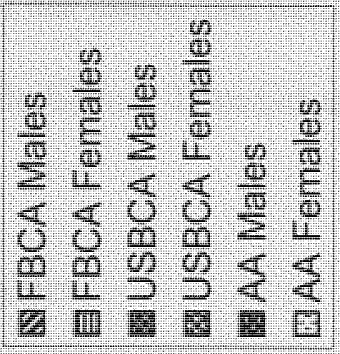

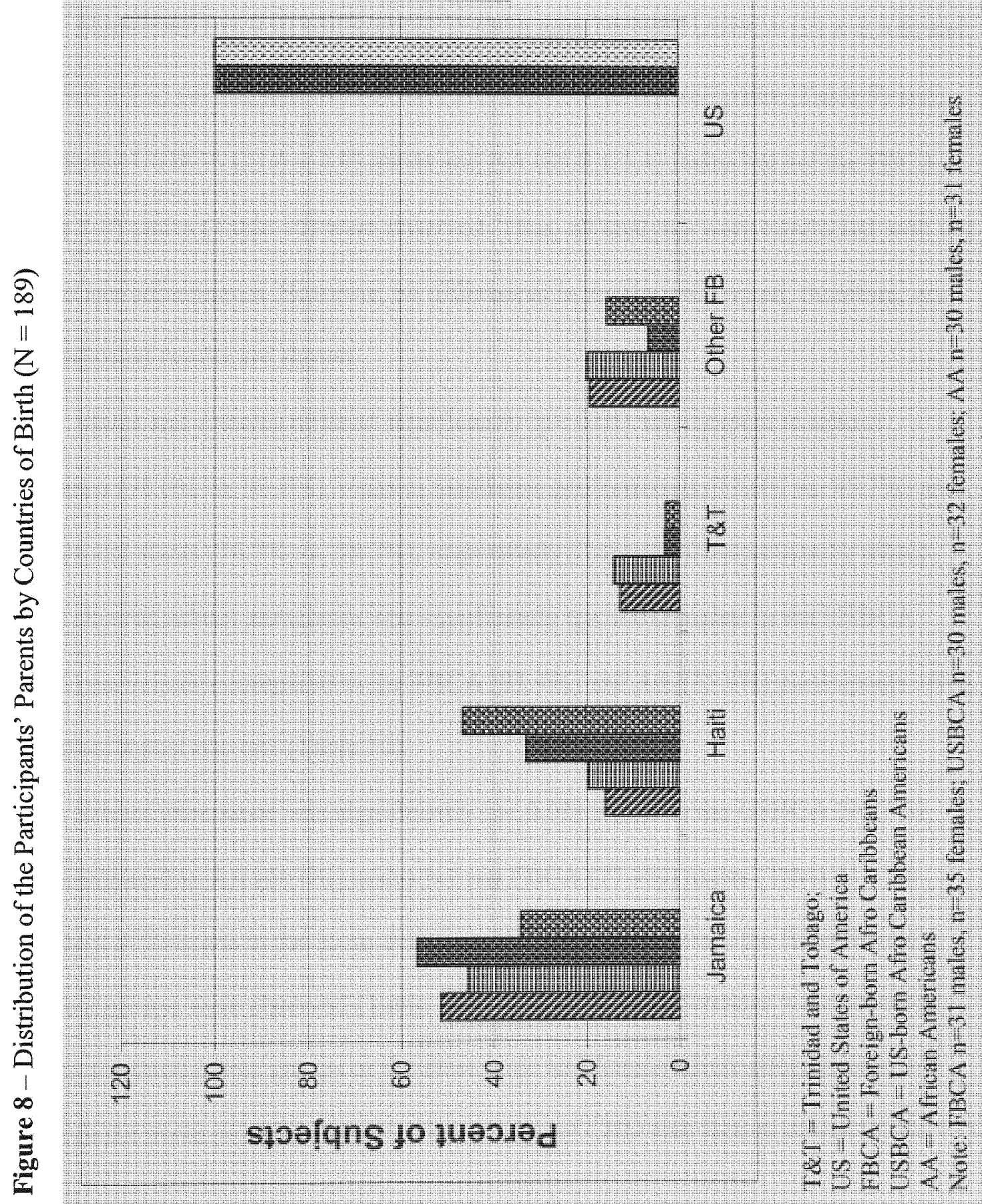


The other parents of the males and females USBCA (10.0\% and $18.7 \%)$, respectively were born in Barbados, Trinidad and Tobago and Guyana (Figure 8).

\section{Socio-demographic Variables}

Significant $(p<0.01)$ differences in mean ages between USBCA $(21.6 \pm 3.8)$ and AA (24.4 \pm 5.1$)$ participants but not the FBCA $(24.3 \pm 5.8)$ participants (Table 9) and, between the USBCA $(21.6 \pm 3.6)$ males and AA $(25.5 \pm 5.4)$ males but not the FBCA $(24.5 \pm 5.8)$ males (Table 10) were observed. Thus, all analyses were conducted with and without age-adjustments. However, no differences in results were noted, therefore, only the unadjusted results are shown.

Males and females differed significantly $(p<0.05)$ with respect to school attendance (78.0\% vs. $90.8 \%$ ), visits to healthcare professionals $(73.6 \%$ vs. $85.7 \%)$ and employment status ( $74.7 \%$ vs. $58.2 \%$ ), respectively (Table 9 ). Comparison by ethnic groups showed school attendance was significantly $(p<0.01)$ higher in the USBCA (96.8\%) participants compared to the FBCA (83.4\%) and AA (73.8\%) participants using Bonferroni's post hoc test (Table 10).

School attendance was significantly $(\mathrm{p}<0.05)$ higher in the USBCA $(96.7 \%)$ males compared to AA (60.0\%) males but not FBCA (77.4\%) males (Table 11). No significant differences in the socio-demographic variables between the three female ethnic subgroups were observed (Table 12). No significant differences were observed between the three ethnic groups or the three male and female ethnic subgroups with respect to the mean perceived stress or knowledge of CHD risk factors scores (Table 13). 
Table 9 - Socio-demographic Factors of Study Participants by Gender

\begin{tabular}{|c|c|c|c|}
\hline Variable & $\begin{array}{l}\text { Males } \\
\mathrm{n}=91\end{array}$ & $\begin{array}{l}\text { Females } \\
\mathbf{n}=98\end{array}$ & $p$ \\
\hline Age (mean \pm SD) & $23.9 \pm 5.3$ & $23.1 \pm 5.0$ & 0.305 \\
\hline \multicolumn{4}{|l|}{ Attend School (\%) } \\
\hline No & 22.0 & 9.2 & $0.038 *$ \\
\hline Yes & 78.0 & 90.8 & \\
\hline \multicolumn{4}{|l|}{ Educational Attainment (\%) } \\
\hline High School Graduate & 71.4 & 74.5 & 0.636 \\
\hline College/Graduate Studies & 28.6 & 25.5 & \\
\hline \multicolumn{4}{|l|}{ Household Income (\%) } \\
\hline$<\$ 20,000$ & 41.8 & 44.7 & 0.971 \\
\hline$\$ 20,000-\$ 39,999$ & 25.3 & 24.9 & \\
\hline$\geq \$ 40,000$ & 33.0 & 30.6 & \\
\hline \multicolumn{4}{|l|}{ Family History of CHD (\%) } \\
\hline No & 30.8 & 28.6 & 0.879 \\
\hline Yes & 33.0 & 31.6 & \\
\hline Do not Know & 36.3 & 39.8 & \\
\hline \multicolumn{4}{|l|}{ Medical Insurance (\%) } \\
\hline No & 26.4 & 34.7 & 0.215 \\
\hline Yes & 73.6 & 65.3 & \\
\hline
\end{tabular}

Visits to Health Care

Professional (\%)

No/never

26.4

14.3

Yes

73.6

85.7

$0.038^{*}$

Employment Status (\%)

Not Employed 25.3

41.8

$0.016^{*}$

Employed

74.7

58.2

Individuals in Household (\%)

$\begin{array}{lll}\leq 3 \text { individuals } & 67.1 & 68.4 \\ >3 \text { individuals } & 32.9 & 31.6\end{array}$

Family History of Diabetes (\%)

$\begin{array}{llll}\text { No } & 20.9 & 8.2 & 0.069 \\ \text { Yes } & 56.0 & 69.4 & \\ \text { Do not Know } & 23.1 & 22.4 & \end{array}$

$* \mathrm{p}<0.05$ 
Table 10 - Socio-demographic Factors of the Participants by Ethnicity

\begin{tabular}{cllll}
\hline Variables & $\begin{array}{l}\text { FBCA } \\
\mathbf{n}=66\end{array}$ & $\begin{array}{l}\text { USBCA } \\
\mathbf{n}=62\end{array}$ & $\begin{array}{l}\text { AA } \\
\mathbf{n}=61\end{array}$ & $\mathbf{p}$ \\
\hline $\begin{array}{c}\text { Age (mean } \pm \text { SD) } \\
\text { Attend School (\%) }\end{array}$ & $24.3 \pm 5.8^{\mathrm{ab}}$ & $21.6 \pm 3.8^{\mathrm{b}}$ & $24.4 \pm 5.1^{\mathrm{a}}$ & $\mathbf{0 . 0 0 2 ^ { * * }}$ \\
No & $16.7^{\mathrm{b}}$ & $3.2^{\mathrm{a}}$ & $26.2^{\mathrm{b}}$ & $\mathbf{0 . 0 0 2 ^ { * * }}$ \\
Yes & 83.4 & 96.8 & 73.8 &
\end{tabular}

Educational Attainment (\%)

High School Graduate

College/Graduate Studies

66.7

82.3

70.5

0.120

33.3

17.7

29.5

Household Income (\%)

$$
\begin{aligned}
& <\$ 20,000 \\
& \$ 20,000-\$ 39,999 \\
& \geq \$ 40,000
\end{aligned}
$$

38.7

45.9

31.1

34.8

24.2

23.0

Family History of CHD (\%)

$\begin{array}{llll}\text { No } & 30.3 & 22.6 & 36.1 \\ \text { Yes } & 39.4 & 25.8 & 31.1 \\ \text { Do not Know } & 30.3 & 51.6 & 32.8\end{array}$

Medical Insurance (\%)

No

Yes

\section{Visits to Health Care}

Professional (\%)

No/never

Yes

Employment Status (\%)

Not Employed

Employed

Individuals in Household (\%)

$\leq 3$ individuals

$>3$ individuals
28.8

71.2

19.7

80.3

31.8

68.2

71.2

28.8

15.2

62.1

22.7
14.5

85.5

30.6

69.4

32.8

67.2
26.2

73.8

0.673

37.1

32.8

Family History of Diabetes (\%)

No

Yes

Do not Know
38.7

61.3

66.2

33.9

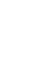


Table 11 - Socio-demographic Factors of the Male Participants by Ethnicity

\begin{tabular}{cllll}
\hline Variables & $\begin{array}{l}\text { FBCA } \\
\mathbf{n}=66\end{array}$ & $\begin{array}{l}\text { USBCA } \\
\mathbf{n}=62\end{array}$ & $\begin{array}{l}\text { AA } \\
\mathbf{n}=61\end{array}$ & $\mathbf{p}$ \\
\hline Age $($ mean \pm SD) & $24.5 \pm 5.8^{\mathrm{ab}}$ & $21.6 \pm 3.6^{\mathrm{b}}$ & $25.5 \pm 5.4^{\mathrm{a}}$ & $\mathbf{0 . 0 0 9 ^ { * * }}$ \\
$\begin{array}{c}\text { Attend School } \\
\text { No }\end{array}$ & $22.6^{\mathrm{ab}}$ & $3.3^{\mathrm{a}}$ & $40.0^{\mathrm{b}}$ & $\mathbf{0 . 0 1 4 ^ { * }}$ \\
Yes & 77.4 & 96.7 & 60.0 &
\end{tabular}

\section{Educational Attainment}

High School Graduate

61.3

83.3

70.0

0.428

College degree

32.3

13.3

26.7

Graduate Studies

6.5

3.3

3.3

Household Income

$\begin{array}{llll}<\$ 20,000 & 51.6 & 33.3 & 40.0 \\ \$ 20,000-\$ 39,999 & 16.2 & 20.0 & 40.0 \\ \geq \$ 40,000 & 32.2 & 46.7 & 20.0\end{array}$

Family History of CHD

No

Yes

Do not Know

Medical Insurance

No

Yes

Visits to Health Care

Professional

No/never

Yes

\section{Employment Status}

Not Employed

Employed

Individuals in Household

$$
\begin{aligned}
& \leq 3 \text { individuals } \\
& >3 \text { individuals }
\end{aligned}
$$

Family History of Diabetes

$\begin{array}{ll}\text { No } & 19.4 \\ \text { Yes } & 54.9\end{array}$

29.0

35.5

35.5

32.3

67.7

22.6

77.4

16.1

83.9

74.2

25.8

19.4

54.9
16.7

40.0

43.3

46.7

23.3

30.0
80.0

16.7

40.0

0.078

83.3

60.0

26.7

$\begin{array}{ll}66.7 & 73.3\end{array}$

0.296

66.7

0.739

33.3

40.0

13.3

30.0

0.434

63.4

${ }^{*} \mathrm{p}<0.05 ;{ }^{* *} \mathrm{p}<0.01$

a,b Values in a row with differing superscripts differed significantly at $\mathrm{p}<0.05$ (Bonferroni's test) FBCA = Foreign-born Afro Caribbeans

USBCA = US-born Afro Caribbean Americans

$\mathrm{AA}=$ African Americans 
Table 12 - Socio-demographic Factors of the Female Participants by Ethnicity

\begin{tabular}{|c|c|c|c|c|}
\hline Variables & $\begin{array}{l}\text { FBCA } \\
n=35\end{array}$ & $\begin{array}{l}\text { USBCA } \\
n=32\end{array}$ & $\begin{array}{l}\text { AA } \\
n=31\end{array}$ & p \\
\hline Age (mean \pm SD) & $24.2 \pm 6.0$ & $21.7 \pm 4.0$ & $23.4 \pm 4.5$ & 0.107 \\
\hline \multicolumn{5}{|l|}{ Attend School (\%) } \\
\hline No & 11.4 & 3.1 & 12.9 & \multirow[t]{2}{*}{0.344} \\
\hline Yes & 88.6 & 96.9 & 87.1 & \\
\hline \multicolumn{5}{|l|}{ Educational Attainment (\%) } \\
\hline High School Graduate & 71.5 & 81.2 & 71.0 & \multirow{3}{*}{0.564} \\
\hline College degree & 20.0 & 15.6 & 19.4 & \\
\hline Graduate Studies & 8.6 & 3.1 & 9.7 & \\
\hline \multicolumn{5}{|l|}{ Household Income (\%) } \\
\hline$<\$ 20,000$ & 40.0 & 43.8 & 51.7 & \multirow[t]{3}{*}{0.812} \\
\hline$\$ 20,000-\$ 39,999$ & 22.8 & 28.2 & 22.6 & \\
\hline$\geq \$ 40,000$ & 37.1 & 28.1 & 25.8 & \\
\hline \multicolumn{5}{|l|}{ Family History of CHD (\%) } \\
\hline No & 31.4 & 28.1 & 25.8 & \multirow[t]{3}{*}{0.136} \\
\hline Yes & 42.9 & 12.5 & 38.7 & \\
\hline Do not Know & 25.7 & 59.4 & 35.5 & \\
\hline \multicolumn{5}{|l|}{ Medical Insurance (\%) } \\
\hline No & 25.7 & 40.6 & 38.7 & \multirow[t]{2}{*}{0.375} \\
\hline Yes & 74.3 & 59.4 & 61.3 & \\
\hline \multicolumn{5}{|l|}{ Visits to Health Care } \\
\hline Professional (\%) & & & & \\
\hline No/never & 17.1 & 12.5 & 12.9 & \multirow[t]{2}{*}{0.237} \\
\hline Yes & 82.9 & 87.5 & 87.1 & \\
\hline \multicolumn{5}{|l|}{ Employment Status (\%) } \\
\hline Not Employed & 45.7 & 43.8 & 35.5 & \multirow[t]{2}{*}{0.678} \\
\hline Employed & 54.3 & 56.3 & 64.5 & \\
\hline \multicolumn{5}{|l|}{ Individuals in Household (\%) } \\
\hline$\leq 3$ individuals & 68.7 & 72.0 & 64.6 & \multirow[t]{2}{*}{0.895} \\
\hline$>3$ individuals & 31.4 & 28.0 & 35.4 & \\
\hline \multicolumn{5}{|l|}{ Family History of Diabetes (\%) } \\
\hline No & 11.4 & 6.3 & 6.5 & \multirow[t]{3}{*}{0.434} \\
\hline Yes & 68.6 & 65.7 & 74.2 & \\
\hline Do not Know & 20.0 & 28.1 & 19.3 & \\
\hline
\end{tabular}

FBCA = Foreign-born Afro Caribbeans

USBCA = US-born Afro Caribbean Americans

$\mathrm{AA}=$ African Americans 
Table 13 - Mean Perceived Stress and Knowledge of Coronary Heart Disease Risk Factor Scores of the Participants by Gender, Ethnicity and Gender-ethnicity Subgroups

\begin{tabular}{|c|c|c|c|}
\hline \multirow[t]{2}{*}{ Variable } & \multicolumn{2}{|c|}{$\begin{array}{l}\text { Perceived Stress } \\
\text { Score }\end{array}$} & \multirow[t]{2}{*}{$\begin{array}{l}\text { KCHDRF } \\
\text { Score }\end{array}$} \\
\hline & $\mathbf{n}$ & & \\
\hline \multicolumn{4}{|l|}{ Gender } \\
\hline Male & 91 & $22.9 \pm 7.8$ & $17.8 \pm 2.1$ \\
\hline Female & 98 & $24.9 \pm 7.1$ & $18.3 \pm 1.6$ \\
\hline Range & & $7-56$ & $0-25$ \\
\hline p-value & & 0.073 & 0.055 \\
\hline \multicolumn{4}{|l|}{ Ethnicity } \\
\hline FBCA & 66 & $24.3 \pm 7.2$ & $17.7 \pm 2.4$ \\
\hline USBCA & 62 & $23.9 \pm 7.5$ & $17.6 \pm 2.1$ \\
\hline $\mathrm{AA}$ & 61 & $23.6 \pm 8.0$ & $18.1 \pm 1.8$ \\
\hline Range & & $7-56$ & $0-25$ \\
\hline p-value & & 0.846 & 0.453 \\
\hline \multicolumn{4}{|c|}{ Males by Ethnicity } \\
\hline FBCA & 31 & $22.5 \pm 7.9$ & $18.0 \pm 1.9$ \\
\hline USBCA & 30 & $22.4 \pm 6.9$ & $18.0 \pm 2.0$ \\
\hline $\mathrm{AA}$ & 30 & $24.0 \pm 8.7$ & $18.3 \pm 1.8$ \\
\hline Range & & $7-56$ & $0-25$ \\
\hline p-value & & 0.665 & 0.674 \\
\hline \multicolumn{4}{|c|}{ Females by Ethnicity } \\
\hline FBCA & 35 & $26.0 \pm 6.1$ & $18.2 \pm 1.4$ \\
\hline USBCA & 32 & $25.4 \pm 7.8$ & $18.3 \pm 1.8$ \\
\hline $\mathrm{AA}$ & 31 & $23.1 \pm 7.3$ & $18.6 \pm 1.7$ \\
\hline Range & & $7-56$ & $0-25$ \\
\hline $\mathrm{p}$-value & & 0.236 & 0.580 \\
\hline
\end{tabular}

Perceived Stress, range $7-56$, where 7 is low stress and 56 is high stress; KCHDRF, Knowledge of Coronary Heart disease Risk Factors, range $0-25$, where 0 is low KCHDRF and 25 is high $\mathrm{KCHDRF}$

FBCA $=$ Foreign-born Afro Caribbeans

USBCA = US-born Afro Caribbean Americans

$\mathrm{AA}=$ African Americans 
Section 2 - Research Question 2: Are FBCA adults less likely to have behavioral/environmental risk factors for CHD as compared to USBCA and AA adults?

\section{Dietary Intakes}

For the dietary analysis, 2 males $(2.2 \%)$ and 2 females (2.0\%) were excluded from the dietary calculations of the data. These participants reported energy intakes either in excess of 7,000 kcal or less than $300 \mathrm{kcal}$ per day, which were outside the reported values by other participants by a large margin in the study.

\section{Energy and Macronutrients}

In general, the mean energy and macronutrient intakes of the males were significantly higher than the females (Table 14). Males consumed more energy (2117.7 \pm $1092.0 \mathrm{kcal}$ vs. $1461.3 \pm 693.5 \mathrm{kcal} ; \mathrm{p}<0.001)$, carbohydrates $(263.6 \pm 134.5 \mathrm{~g}$ vs. 201.8 $\pm 100.3 \mathrm{~g} ; \mathrm{p}<0.001)$, protein $(84.5 \pm 47.0 \mathrm{~g}$ vs. $53.6 \pm 26.3 \mathrm{~g} ; \mathrm{p}<0.001)$, total fat $(78.6 \pm$ 48.2 g vs. $49.8 \pm 28.0$ g; $p<0.001)$, saturated fat $(27.3 \pm 16.4$ g vs. $17.3 \pm 9.9$ g; $p<$ $0.001)$, monounsaturated fatty acid (MUFA) $(30.4 \pm 19.6$ g vs. $18.3 \pm 10.7 \mathrm{~g} ; \mathrm{p}<0.001)$, polyunsaturated fatty acid (PUFA) $(13.8 \pm 9.3 \mathrm{~g}$ vs. $9.8 \pm 6.2 \mathrm{~g} ; \mathrm{p}<0.001)$, cholesterol $(271.4 \pm 167.7 \mathrm{mg}$ vs. $152.8 \pm 95.0 \mathrm{mg} ; \mathrm{p}<0.01)$ and caffeine $(25.8 \pm 31.5 \mathrm{mg}$ vs. $17.1 \pm$ $23.4 \mathrm{mg} ; \mathrm{p}<0.05$ ) than the females. 
Table 14 - Mean Energy and Nutrient Intakes of the Participants by Gender

\begin{tabular}{|c|c|c|c|}
\hline \multirow[t]{2}{*}{ Variables } & Males & Females & \multirow[t]{2}{*}{$\mathbf{p}$} \\
\hline & \multicolumn{2}{|c|}{ Males $\quad$ Mean \pm SD } & \\
\hline Calories (Kcal) & $2117.7 \pm 1092.0$ & $1461.3 \pm 693.5$ & $0.0001 * * *$ \\
\hline $\mathrm{CHO}^{1}(\mathrm{~g})$ & $263.6 \pm 134.5$ & $201.8 \pm 100.3$ & $0.0001 * * *$ \\
\hline Protein (g) & $84.5 \pm 47.0$ & $53.6 \pm 26.3$ & $0.0001 * * *$ \\
\hline Total Fat (g) & $78.6 \pm 48.2$ & $49.8 \pm 28.0$ & $0.0001 * * *$ \\
\hline $\mathrm{SF}^{2}(\mathrm{~g})$ & $27.3 \pm 16.4$ & $17.3 \pm 9.9$ & $0.0001 * * *$ \\
\hline $\operatorname{MUFA}^{3}(\mathrm{~g})$ & $30.4 \pm 19.6$ & $18.3 \pm 10.7$ & $0.0001 * * *$ \\
\hline $\mathrm{PUFA}^{4}(\mathrm{~g})$ & $13.8 \pm 9.3$ & $9.8 \pm 6.2$ & $0.001 * *$ \\
\hline Cholesterol (mg) & $271.4 \pm 167.7$ & $152.8 \pm 95.0$ & $0.0001 * * *$ \\
\hline $\mathrm{Kcal} \mathrm{CHO}^{1}(\%)$ & $51.3 \pm 11.3$ & $55.3 \pm 8.2$ & $0.006^{* *}$ \\
\hline Kcal Prot (\%) & $16.1 \pm 4.1$ & $14.9 \pm 3.4$ & $0.034^{*}$ \\
\hline Kcal Fat (\%) & $32.2 \pm 7.2$ & $30.3 \pm 6.2$ & $0.050^{*}$ \\
\hline Kcal Alcohol (\%) & $2.7 \pm 4.3$ & $1.9 \pm 4.7$ & 0.234 \\
\hline Kcal Sweet (\%) & $11.4 \pm 8.9$ & $11.3 \pm 7.9$ & 0.917 \\
\hline Fiber $(\mathrm{g})$ & $16.5 \pm 9.6$ & $14.2 \pm 10.2$ & 0.115 \\
\hline Fiber (Beans) (g) & $2.9 \pm 4.5$ & $2.7 \pm 3.6$ & 0.685 \\
\hline Fiber (Vegetables) (g) & $6.5 \pm 5.1$ & $6.2 \pm 5.9$ & 0.675 \\
\hline Fiber (Grains) (g) & $7.3 \pm 4.4$ & $5.4 \pm 3.5$ & $0.001^{* *}$ \\
\hline Caffeine (mg) & $25.8 \pm 31.4$ & $17.1 \pm 23.4$ & $0.032 *$ \\
\hline Fruits (servings/d) & $1.1 \pm 0.7$ & $1.3 \pm 0.9$ & $0.035^{*}$ \\
\hline Vegetables (servings/d) & $2.4 \pm 2.4$ & $2.2 \pm 2.1$ & 0.550 \\
\hline Grains (servings/d) & $5.1 \pm 2.9$ & $4.1 \pm 2.6$ & $0.016^{*}$ \\
\hline Meats (servings/d) & $2.9 \pm 2.0$ & $1.7 \pm 1.1$ & $0.0001 * * *$ \\
\hline Dairy (servings/d) & $1.5 \pm 1.2$ & $1.0 \pm 0.8$ & $0.003^{* *}$ \\
\hline Fats (servings/d) & $1.6 \pm 1.2$ & $1.4 \pm 0.8$ & 0.115 \\
\hline
\end{tabular}

${ }^{*} \mathrm{p}<0.05 ;{ }^{* *} \mathrm{p}<0.01 ; * * * \mathrm{p}<0.001$

${ }^{1} \mathrm{CHO}=$ Carbohydrate; ${ }^{2} \mathrm{SF}=$ Saturated Fat; ${ }^{3}$ MUFA $=$ Monounsaturated Fatty Acids; ${ }^{4} \mathrm{PUFA}=$ Polyunsaturated Fatty Acids 
Males consumed significantly less energy from carbohydrates $(51.3 \pm 11.3 \%$ vs. $55.3 \pm$ $8.2 \% ; \mathrm{p}<0.01)$, but significantly more energy from protein $(16.1 \pm 4.1 \%$ vs. $14.9 \pm$ $3.4 \% ; p<0.05)$ and fat $(32.2 \pm 7.2 \%$ vs. $30.3 \pm 6.2 \% ; p=0.050)$ compared to females.

Males also consumed more fiber from grains than females $(7.3 \pm 4.4 \mathrm{~g}$ vs. $5.4 \pm 3.5 \mathrm{~g}$; $\mathrm{p}<$ 0.01). The consumption of foods from the food groups, grains ( $5.1 \pm 2.9$ servings vs. 4.1 \pm 2.6 servings; $p<0.05)$, meats $(2.9 \pm 2.0$ servings vs. $1.7 \pm 1.1$ servings; $p<0.001)$ and dairy $(1.5 \pm 1.2$ servings vs. $1.0 \pm 0.8$ servings; $p<0.01)$ were also significantly higher in males than females. However, males consumed significantly fewer serving of fruits per day compared to females $(1.1 \pm 0.7$ servings vs. $1.3 \pm 0.9$ servings; $\mathrm{p}<0.05)$ (Table 14$)$.

The FBCA participants compared to USBCA and AA participants consumed significantly less protein $(57.6 \pm 32.2 \mathrm{~g}$ vs. $74.0 \pm 44.1 \mathrm{~g}$ and $74.7 \pm 43.3 \mathrm{~g} ; \mathrm{p}<0.05)$,

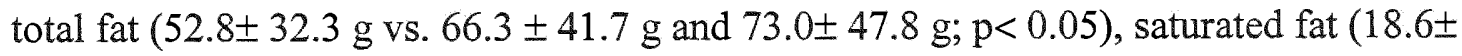
$11.5 \mathrm{~g}$ vs. $23.1 \pm 14.9 \mathrm{~g}$ and $24.9 \pm 15.8 \mathrm{~g} ; \mathrm{p}<0.05)$, MUFA $(19.7 \pm 13.3 \mathrm{~g}$ vs. $25.1 \pm$ $17.1 \mathrm{~g}$ and $27.9 \pm 18.9 \mathrm{~g} ; \mathrm{p}<0.05)$, PUFA $(9.5 \pm 5.9 \mathrm{~g}$ vs. $12.0 \pm 7.5 \mathrm{~g}$ and $13.9 \pm 10.0 \mathrm{~g}$; $\mathrm{p}<0.05)$, cholesterol (168.8 $\pm 114.0 \mathrm{mg}$ vs. $220.4 \pm 161.9 \mathrm{mg}$ and $244.1 \pm 155.0 \mathrm{mg} ; \mathrm{p}<$ $0.05)$ and meat serving per day ( $1.7 \pm 1.3$ servings vs. $2.6 \pm 1.9$ servings and $2.5 \pm 1.8$ servings; $\mathrm{p}<0.01$ ). In addition, the FBCA participants compared to the USBCA and AA participants consumed significantly more energy from carbohydrate $(56.7 \pm 10.2 \%$ vs. $52.7 \pm 10.2 \%$ and $50.6 \pm 8.5 \% ; \mathrm{p}<0.05)$. However, the FBCA participants compared to the AA but not the USBCA participants consumed significantly less energy from fat $(29.3 \pm 6.8 \%$ vs. $33.1 \pm 6.5 \%$ and $31.4 \pm 6.4 \% ; p<0.01)$ and fat servings $(1.2 \pm 0.9$ servings vs. $1.8 \pm 1.2$ servings and $1.5 \pm 1.0$ servings; $\mathrm{p}<0.05$ ), respectively (Table 15 ). 
Table 15 - Mean Energy and Nutrient Intakes of the Participants by Ethnicity

\begin{tabular}{|c|c|c|c|c|}
\hline Variables & 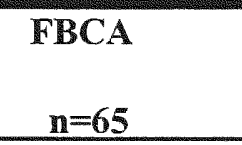 & $\begin{array}{c}\text { USBCA } \\
\text { Mean } \pm \text { SD } \\
n=61\end{array}$ & $\begin{array}{l}\mathrm{AA} \\
\mathrm{n}=59\end{array}$ & $\mathbf{p}$ \\
\hline$\overline{\text { Calories (Kcal) }}$ & $1557.9 \pm 775.2$ & $1843.4 \pm 976.8$ & $1950.1 \pm 1096.0$ & 0.061 \\
\hline $\mathrm{CHO}^{1}(\mathrm{~g})$ & $216.4 \pm 102.4$ & $235.1 \pm 120.8$ & $244.5 \pm 140.8$ & 0.423 \\
\hline Protein $(\mathrm{g})$ & $57.6 \pm 32.2^{\mathrm{a}}$ & $74.0 \pm 44.1^{\mathrm{b}}$ & $74.7 \pm 43.3^{b}$ & $0.027^{*}$ \\
\hline Total Fat (g) & $52.8 \pm 32.3^{\mathrm{a}}$ & $66.3 \pm 41.7^{b}$ & $73.0 \pm 47.8^{b}$ & $0.020^{*}$ \\
\hline $\mathrm{SF}^{2}(\mathrm{~g})$ & $18.6 \pm 11.5^{\mathrm{a}}$ & $23.1 \pm 14.9^{\mathrm{b}}$ & $24.9 \pm 15.8^{\mathrm{b}}$ & $0.042^{*}$ \\
\hline $\operatorname{MUFA}^{3}(\mathrm{~g})$ & $19.7 \pm 13.3^{\mathrm{a}}$ & $25.1 \pm 17.1^{b}$ & $27.9 \pm 18.9^{\mathrm{b}}$ & $0.019^{*}$ \\
\hline $\mathrm{PUFA}^{4}(\mathrm{~g})$ & $9.5 \pm 5.9^{\mathrm{a}}$ & $12.0 \pm 7.5^{\mathrm{b}}$ & $13.9 \pm 10.0^{b}$ & $0.011^{*}$ \\
\hline Cholesterol (mg) & $168.8 \pm 114.0^{\mathrm{a}}$ & $220.4 \pm 161.9^{\mathrm{b}}$ & $244.1 \pm 155.0^{\mathrm{b}}$ & $0.013^{*}$ \\
\hline $\mathrm{Kcal} \mathrm{CHO}^{1}(\%)$ & $56.7 \pm 10.2^{\mathrm{a}}$ & $52.7 \pm 10.2^{\mathrm{b}}$ & $50.6 \pm 8.5^{\mathrm{b}}$ & $0.002 * *$ \\
\hline Kcal Prot (\%) & $14.9 \pm 4.1$ & $15.9 \pm 3.6$ & $15.7 \pm 3.6$ & 0.282 \\
\hline Kcal Fat (\%) & $29.3 \pm 6.8^{\mathrm{a}}$ & $31.4 \pm 6.4^{a b}$ & $33.1 \pm 6.5^{\mathrm{b}}$ & $0.008^{* *}$ \\
\hline Kcal Alcohol (\%) & $1.7 \pm 4.0$ & $2.1 \pm 4.1$ & $3.1 \pm 5.3$ & 0.215 \\
\hline Kcal Sweet (\%) & $11.3 \pm 10.0$ & $10.6 \pm 6.4$ & $12.2 \pm 8.4$ & 0.597 \\
\hline Fiber $(\mathrm{g})$ & $15.6 \pm 10.7$ & $15.0 \pm 9.9$ & $15.3 \pm 9.2$ & 0.954 \\
\hline Fiber (Beans) (g) & $3.0 \pm 4.6$ & $2.9 \pm 3.9$ & $2.5 \pm 3.5$ & 0.773 \\
\hline Fiber (Vegetables) (g) & $6.7 \pm 5.8$ & $5.9 \pm 6.1$ & $6.4 \pm 4.6$ & 0.771 \\
\hline Fiber (Grains) (g) & $6.1 \pm 4.1$ & $6.2 \pm 3.9$ & $6.6 \pm 4.2$ & 0.712 \\
\hline Caffeine (mg) & $18.4 \pm 26.2$ & $21.0 \pm 24.6$ & $24.8 \pm 32.3$ & 0.440 \\
\hline Fruits (servings/d) & $1.4 \pm 0.9$ & $1.2 \pm 0.8$ & $1.1 \pm 0.6$ & 0.107 \\
\hline Vegetables (servings/d) & $2.3 \pm 2.3$ & $2.2 \pm 2.3$ & $2.5 \pm 2.1$ & 0.791 \\
\hline Grains (servings/d) & $4.4 \pm 2.9$ & $4.7 \pm 2.7$ & $4.7 \pm 2.9$ & 0.845 \\
\hline Meats (servings/d) & $1.7 \pm 1.3^{\mathrm{a}}$ & $2.6 \pm 1.9^{b}$ & $2.5 \pm 1.8^{b}$ & $0.009 * *$ \\
\hline Dairy (servings/d) & $1.2 \pm 1.1$ & $1.3 \pm 1.0$ & $1.2 \pm 1.0$ & 0.937 \\
\hline Fats (servings/d) & $1.2 \pm 0.9^{\mathrm{a}}$ & $1.5 \pm 1.0^{\mathrm{ab}}$ & $1.8 \pm 1.2^{b}$ & $0.015^{*}$ \\
\hline
\end{tabular}

${ }^{*} \mathrm{p}<0.05 ; * \mathrm{p}<0.01$

${ }^{a, b}$ Values in a row with differing superscripts differed significantly at $p<0.05$ (Bonferroni's test).

${ }^{1} \mathrm{CHO}=$ Carbohydrate $;{ }^{2} \mathrm{SF}=$ Saturated Fat $;{ }^{3}$ MUFA $=$ Monounsaturated Fatty Acids; ${ }^{4} \mathrm{PUFA}=$ Polyunsaturated Fatty Acids

$\mathrm{FBCA}=$ Foreign-born Afro Caribbeans

USBCA = US-born Afro Caribbean Americans

$\mathrm{AA}=$ African Americans 
In the male ethnic subgroups, the FBCA males compared to the USBCA and AA males, consumed significantly less energy $(1721.9 \pm 883.2 \mathrm{kcal}$ vs. $2301.3 \pm 1016.0 \mathrm{kcal}$ and $2337.3 \pm 1271.6 \mathrm{kcal} ; \mathrm{p}<0.05)$, protein $(66.9 \pm 38.0 \mathrm{~g}$ vs. $95.5 \pm 48.1 \mathrm{~g}$ and $91.3 \pm$ $50.4 \mathrm{~g} ; \mathrm{p}<0.05)$, total fat $(59.2 \pm 37.5 \mathrm{~g}$ vs. $86.7 \pm 47.0 \mathrm{~g}$ and $90.3 \pm 54.4 \mathrm{~g} ; \mathrm{p}<0.05)$, saturated fat $(21.4 \pm 13.4 \mathrm{~g}$ vs. $30.3 \pm 17.1 \mathrm{~g}$ and $30.5 \pm 17.4 \mathrm{~g} ; \mathrm{p}<0.05)$, MUFA $(22.5 \pm$ $15.8 \mathrm{~g}$ vs. $33.8 \pm 19.5 \mathrm{~g}$ and $35.1 \pm 21.5 \mathrm{~g} ; \mathrm{p}<0.05)$ and PUFA $(9.7 \pm 5.7 \mathrm{~g}$ vs. $14.8 \pm 7.9$ $\mathrm{g}$ and $17.0 \pm 11.9 \mathrm{~g} ; \mathrm{p}<0.01$ ) (Table 15). In addition, the FBCA males, compared to the USBCA and AA males, consumed significantly more energy from carbohydrates (56.0 \pm $12.2 \%$ vs. $49.5 \pm 11.7 \%$ and $48.4 \pm 8.1 \% ; p<0.05)$. The percentage energy intake from fat of the FBCA males $(29.4 \pm 7.6 \%)$ was significantly $(\mathrm{p}<0.05)$ lower compared to the AA (34.6 $\pm 5.5 \%)$ but not the USBCA males. The FBCA males ( $2.1 \pm 1.5$ servings $)$ compared to the USBCA ( $3.4 \pm 2.2$ servings $)$ but not the AA males $(3.2 \pm 2.2)$ consumed significantly less $(p<0.05)$ meat servings, whereas fat servings were significantly lower in the FBCA males compared to the USBCA and AA males $(1.2 \pm 1.0$ servings vs. $1.9 \pm$ 1.2 servings and $1.9 \pm 1.4$ servings; $p<0.05)$ (Table 16$)$.

In the female ethnic subgroups, the only difference observed was that of the FBCA females $(132.6 \pm 78.6 \mathrm{mg})$ compared to the AA $(188.4 \pm 126.7 \mathrm{mg})$ but not the USBCA females $(141.2 \pm 64.7 \mathrm{mg})$ consumed significantly $(\mathrm{p}<0.05)$ less dietary cholesterol (Table 17). 
Table 16 - Mean Energy and Nutrient Intakes of Males by Ethnicity

\begin{tabular}{|c|c|c|c|c|}
\hline Variables & $\begin{array}{l}\text { FBCA } \\
n=30 \\
\end{array}$ & $\begin{array}{c}\text { USBCA } \\
\text { Mean } \pm \text { SD } \\
n=30 \\
\end{array}$ & $\begin{array}{l}\text { AA } \\
n=29 \\
\end{array}$ & $\mathbf{p}$ \\
\hline Calories (Kcal) & $1721.9 \pm 883.2^{\mathrm{a}}$ & $2301.3 \pm 1016.0^{\mathrm{b}}$ & $2337.3 \pm 1271.6^{b}$ & $0.049^{*}$ \\
\hline $\mathrm{CHO}^{1}(\mathrm{~g})$ & $234.1 \pm 112.9$ & $273.8 \pm 118.9$ & $283.5 \pm 165.9$ & 0.328 \\
\hline Protein (g) & $66.9 \pm 38.0^{\mathrm{a}}$ & $95.5 \pm 48.1^{\mathrm{b}}$ & $91.3 \pm 50.4^{b}$ & $0.037 *$ \\
\hline Total Fat (g) & $59.2 \pm 37.4^{\mathrm{a}}$ & $86.7 \pm 47.0^{b}$ & $90.3 \pm 54.4^{\mathrm{b}}$ & $0.023^{*}$ \\
\hline $\mathrm{SF}^{2}(\mathrm{~g})$ & $21.4 \pm 13.4^{\mathrm{a}}$ & $30.3 \pm 17.1^{\mathrm{b}}$ & $30.5 \pm 17.4^{b}$ & $0.048^{*}$ \\
\hline $\operatorname{MUFA}^{3}(\mathrm{~g})$ & $22.5 \pm 15.6^{\mathrm{a}}$ & $33.8 \pm 19.5^{b}$ & $35.1 \pm 21.5^{\mathrm{b}}$ & $0.023^{*}$ \\
\hline $\mathrm{PUFA}^{4}(\mathrm{~g})$ & $9.7 \pm 5.7^{\mathrm{a}}$ & $14.8 \pm 7.9^{\mathrm{b}}$ & $17.0 \pm 11.9^{\mathrm{b}}$ & $0.006^{* *}$ \\
\hline Cholesterol (mg) & $211.2 \pm 134.1$ & $302.3 \pm 190.4$ & $301.7 \pm 162.4$ & 0.052 \\
\hline $\mathrm{Kcal} \mathrm{CHO}^{1}(\%)$ & $56.0 \pm 12.2^{\mathrm{a}}$ & $49.5 \pm 11.7^{b}$ & $48.4 \pm 8.1^{b}$ & $0.017 *$ \\
\hline Kcal Prot (\%) & $15.8 \pm 5.0$ & $16.4 \pm 3.6$ & $16.0 \pm 3.6$ & 0.873 \\
\hline Kcal Fat (\%) & $29.4 \pm 7.6^{\mathrm{a}}$ & $32.8 \pm 7.3^{\mathrm{ab}}$ & $34.6 \pm 5.5^{b}$ & $0.015^{*}$ \\
\hline Kcal Alcohol (\%) & $1.2 \pm 2.7$ & $3.6 \pm 5.4$ & $3.2 \pm 3.9$ & 0.058 \\
\hline Kcal Sweet (\%) & $10.6 \pm 10.7$ & $10.9 \pm 5.9$ & $12.8 \pm 9.6$ & 0.600 \\
\hline Fiber $(\mathrm{g})$ & $16.6 \pm 10.3$ & $15.5 \pm 7.0$ & $17.4 \pm 11.3$ & 0.757 \\
\hline Fiber (Beans) (g) & $3.7 \pm 5.7$ & $2.1 \pm 3.0$ & $3.0 \pm 4.3$ & 0.403 \\
\hline Fiber (Vegetables) (g) & $6.6 \pm 5.3$ & $5.9 \pm 4.2$ & $7.2 \pm 5.7$ & 0.641 \\
\hline Fiber (Grains) (g) & $6.8 \pm 4.1$ & $7.5 \pm 4.3$ & $7.6 \pm 4.9$ & 0.744 \\
\hline Caffeine (mg) & $20.0 \pm 32.9$ & $29.3 \pm 25.1$ & $28.2 \pm 35.7$ & 0.462 \\
\hline Fruits (servings/d) & $1.2 \pm 0.9$ & $1.0 \pm 0.6$ & $0.9 \pm 0.6$ & 0.229 \\
\hline Vegetables (servings/d) & $2.3 \pm 2.6$ & $2.2 \pm 2.1$ & $2.8 \pm 2.5$ & 0.590 \\
\hline Grains (servings/d) & $4.9 \pm 2.8$ & $5.1 \pm 2.6$ & $5.5 \pm 3.5$ & 0.838 \\
\hline Meats (servings/d) & $2.1 \pm 1.5^{\mathrm{a}}$ & $3.4 \pm 2.2^{\mathrm{b}}$ & $3.2 \pm 2.2^{\mathrm{ab}}$ & $0.034^{*}$ \\
\hline Dairy (servings/d) & $1.5 \pm 1.2$ & $1.5 \pm 1.0$ & $1.4 \pm 1.3$ & 0.964 \\
\hline Fats (servings/d) & $1.2 \pm 1.0^{\mathrm{a}}$ & $1.9 \pm 1.2^{\mathrm{b}}$ & $1.9 \pm 1.4^{\mathrm{b}}$ & $0.042^{*}$ \\
\hline
\end{tabular}

${ }^{*} \mathrm{p}<0.05 ;{ }^{* *} \mathrm{p}<0.01$

${ }^{a, b}$ Values in a row with differing superscripts differed significantly at $\mathrm{p}<0.05$ (Bonferroni's test)

${ }^{1} \mathrm{CHO}=$ Carbohydrate $;{ }^{2} \mathrm{SF}=$ Saturated Fat; ${ }^{3}$ MUFA $=$ Monounsaturated Fatty Acids; $;{ }^{4} \mathrm{PUFA}=$ Polyunsaturated Fatty Acids

FBCA $=$ Foreign-born Afro Caribbeans

USBCA = US-born Afro Caribbean Americans

$\mathrm{AA}=$ African Americans 
Table 17 - Mean Energy and Nutrient Intakes of Females by Ethnicity

\begin{tabular}{|c|c|c|c|c|}
\hline Variables & $\begin{array}{l}\text { FBCA } \\
n=35\end{array}$ & $\begin{array}{c}\text { USBCA } \\
\text { Mean } \pm \text { SD } \\
n=31\end{array}$ & $\begin{array}{l}\mathrm{AA} \\
\mathrm{n}=30\end{array}$ & $\mathrm{p}$ \\
\hline Calories (Kcal) & $1417.4 \pm 649.5$ & $1400.2 \pm 705.5$ & $1575.8 \pm 739.1$ & 0.554 \\
\hline $\mathrm{CHO}^{1}(\mathrm{~g})$ & $201.2 \pm 91.4$ & $197.6 \pm 112.1$ & $206.8 \pm 100.5$ & 0.938 \\
\hline Protein $(\mathrm{g})$ & $49.6 \pm 24.1$ & $53.1 \pm 27.2$ & $58.8 \pm 27.7$ & 0.374 \\
\hline Total Fat (g) & $47.3 \pm 26.5$ & $46.5 \pm 23.1$ & $56.3 \pm 33.6$ & 0.314 \\
\hline $\mathrm{SF}^{2}(\mathrm{~g})$ & $16.3 \pm 9.2$ & $16.2 \pm 8.0$ & $19.5 \pm 12.2$ & 0.338 \\
\hline MUFA $^{3}(\mathrm{~g})$ & $17.3 \pm 10.4$ & $16.6 \pm 8.2$ & $21.1 \pm 13.0$ & 0.220 \\
\hline $\mathrm{PUFA}^{4}(\mathrm{~g})$ & $9.4 \pm 6.2$ & $9.3 \pm 6.0$ & $10.8 \pm 6.6$ & 0.596 \\
\hline Cholesterol (mg) & $132.6 \pm 78.6^{\mathrm{a}}$ & $141.2 \pm 64.7^{\mathrm{ab}}$ & $188.4 \pm 126.7^{\mathrm{b}}$ & $0.042 *$ \\
\hline $\mathrm{Kcal} \mathrm{CHO}^{1}(\%)$ & $57.3 \pm 8.2$ & $55.8 \pm 7.4$ & $52.6 \pm 8.4$ & 0.065 \\
\hline Kcal Prot (\%) & $14.0 \pm 3.0$ & $15.3 \pm 3.5$ & $15.4 \pm 3.7$ & 0.176 \\
\hline Kcal Fat (\%) & $29.3 \pm 6.2$ & $30.2 \pm 5.2$ & $31.6 \pm 7.1$ & 0.340 \\
\hline Kcal Alcohol (\%) & $2.1 \pm 4.8$ & $0.6 \pm 1.0$ & $3.0 \pm 3.9$ & 0.143 \\
\hline Kcal Sweet (\%) & $11.9 \pm 9.4$ & $10.3 \pm 6.9$ & $11.6 \pm 6.5$ & 0.714 \\
\hline Fiber $(\mathrm{g})$ & $14.7 \pm 11.2$ & $14.6 \pm 12.3$ & $13.3 \pm 6.2$ & 0.831 \\
\hline Fiber (Beans) (g) & $2.5 \pm 3.4$ & $3.6 \pm 4.5$ & $2.1 \pm 2.5$ & 0.245 \\
\hline Fiber (Vegetables) (g) & $6.9 \pm 6.3$ & $5.9 \pm 7.6$ & $5.7 \pm 3.0$ & 0.687 \\
\hline Fiber (Grains) (g) & $5.4 \pm 4.0$ & $5.0 \pm 3.2$ & $5.7 \pm 3.2$ & 0.753 \\
\hline Caffeine (mg) & $17.0 \pm 19.1$ & $12.9 \pm 21.7$ & $21.5 \pm 28.9$ & 0.362 \\
\hline Fruits (servings/d) & $1.5 \pm 0.9$ & $1.3 \pm 1.0$ & $1.2 \pm 0.6$ & 0.440 \\
\hline Vegetables (servings/d) & $2.3 \pm 2.0$ & $2.2 \pm 2.6$ & $2.2 \pm 1.5$ & 0.958 \\
\hline Grains (servings/d) & $4.1 \pm 3.0$ & $4.2 \pm 2.7$ & $4.1 \pm 2.2$ & 0.975 \\
\hline Meats (servings/d) & $1.4 \pm 1.0$ & $1.7 \pm 1.1$ & $1.9 \pm 1.1$ & 0.150 \\
\hline Dairy (servings/d) & $1.0 \pm 0.9$ & $1.0 \pm 0.9$ & $1.0 \pm 0.7$ & 0.967 \\
\hline Fats (servings/d) & $1.3 \pm 0.7$ & $1.2 \pm 0.7$ & $1.7 \pm 1.0$ & 0.079 \\
\hline
\end{tabular}

$* \mathrm{p}<0.05$

${ }^{\mathrm{a}, \mathrm{b}}$ Values in a row with differing superscripts differed significantly at $\mathrm{p}<0.05$ (Bonferroni's test) ${ }^{1} \mathrm{CHO}=$ Carbohydrate; ${ }^{2} \mathrm{SF}=$ Saturated Fat; ${ }^{3}$ MUFA $=$ Monounsaturated Fatty Acids; ${ }^{4}$ PUFA $=$ Polyunsaturated Fatty Acids

$\mathrm{FBCA}=$ Foreign-born Afro Caribbeans

USBCA = US-born Afro Caribbean Americans

$\mathrm{AA}=$ African Americans 


\section{Mineral Intakes}

The mean sodium ( $2555.0 \pm 1365.6 \mathrm{mg}$ vs. $1779.0 \pm 916.4 \mathrm{mg} ; \mathrm{p}<001)$, potassium (3005.3 $\pm 1470.2 \mathrm{mg}$ vs. $2275.2 \pm 1136.9 \mathrm{mg} ; \mathrm{p}<0.001)$, calcium $724.7 \pm$ $408.9 \mathrm{mg}$ vs. $546.8 \pm 311.0 \mathrm{mg} ; \mathrm{p}<0.01)$, iron (15.5 $\pm 7.2 \mathrm{mg}$ vs. $11.3 \pm 6.4 \mathrm{mg} ; \mathrm{p}<$ $0.001)$, magnesium (292.1 $\pm 150.2 \mathrm{mg}$ vs. $220.4 \pm 116.2 \mathrm{mg} ; \mathrm{p}<0.001)$, zinc $(11.4 \pm 6.4$ mg vs. $7.2 \pm 4.1 \mathrm{mg} ; \mathrm{p}<0.001)$ and phosphorus intakes $(1237.7 \pm 621.9 \mathrm{mg}$ vs. $847.6 \pm$ $410.6 \mathrm{mg} ; \mathrm{p}<0.001$ ) of the males were significantly higher than those of the females. There were no significant differences between the gender groups with respect to mineral intakes from supplements (Table 18).

With respect to the three ethnic groups, the mean sodium intake of the FBCA participants $(1838.0 \pm 983.4 \mathrm{mg})$ was significantly $(\mathrm{p}<0.05)$ lower compared to AA (2402.6 $\pm 1359.3 \mathrm{mg})$ but not USBCA $(2245.2 \pm 1238.3 \mathrm{mg})$ participants. However, the mean iron intake of the FBCA participants $(11.2 \pm 5.6 \mathrm{mg})$ was significantly $(\mathrm{p}<0.01)$ lower than USBCA $(15.0 \pm 8.1 \mathrm{mg})$ but not AA participants $(13.8 \pm 7.0 \mathrm{mg})$ (Table 19). The mean sodium $(2031.7 \pm 1101.3 \mathrm{mg}$ vs. $2760.6 \pm 1296.7 \mathrm{mg}$ and $2883.7 \pm 1553.4 \mathrm{mg}$; $\mathrm{p}<0.05)$ and iron intakes $(12.7 \pm 6.0 \mathrm{mg}$ vs. $17.3 \pm 7.0 \mathrm{mg}$ and $16.4 \pm 7.9 \mathrm{mg} ; \mathrm{p}<0.05)$ of the FBCA males were significantly lower compared to the USBCA and AA males. There was no significant difference observed between the male ethnic subgroups with respect to the consumption of minerals from supplements (Table 20). There were no significant differences between the three female ethnic subgroups with respect to the consumption of minerals or minerals from supplements (Table 21). 
Table 18 - Mean Mineral Intakes of the Participants by Gender

\begin{tabular}{|c|c|c|c|}
\hline \multirow[t]{2}{*}{ Variables } & Males & Females & \multirow[t]{2}{*}{$\mathbf{p}$} \\
\hline & \multicolumn{2}{|c|}{ Mean \pm SD } & \\
\hline 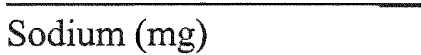 & $2555.0 \pm 1365.6$ & $1779.0 \pm 916.4$ & $0.0001^{* * * *}$ \\
\hline Potassium (mg) & $3005.3 \pm 1470.2$ & $2275.2 \pm 1136.9$ & $0.0001^{* * * *}$ \\
\hline Calcium (mg) & $724.7 \pm 408.9$ & $546.8 \pm 311.0$ & $0.001^{* *}$ \\
\hline Iron (mg) & $15.5 \pm 7.2$ & $11.3 \pm 6.4$ & $0.0001 * * *$ \\
\hline Magnesium (mg) & $292.1 \pm 150.2$ & $220.4 \pm 116.2$ & $0.0001 * * *$ \\
\hline Zinc (mg) & $11.4 \pm 6.4$ & $7.2 \pm 4.1$ & $0.0001 * * *$ \\
\hline Phosphorus (mg) & $1237.7 \pm 621.9$ & $847.6 \pm 410.6$ & $0.0001 * * *$ \\
\hline From Supplements Only & $\mathrm{n}=40$ & $\mathrm{n}=40$ & \\
\hline Calcium (mg) & $65.9 \pm 154.0$ & $91.6 \pm 191.8$ & 0.318 \\
\hline Iron (mg) FS ${ }^{\#}$ & $7.1 \pm 12.5$ & $10.4 \pm 18.8$ & 0.170 \\
\hline Magnesium (mg) & $21.0 \pm 30.6$ & $28.1 \pm 39.4$ & 0.175 \\
\hline Zinc (mg) & $4.8 \pm 8.8$ & $4.7 \pm 6.4$ & 0.876 \\
\hline Copper (mg) & $0.4 \pm 0.6$ & $0.6 \pm 0.8$ & 0.187 \\
\hline Selenium (mg) & $11.6 \pm 29.5$ & $7.3 \pm 12.2$ & 0.184 \\
\hline
\end{tabular}

** $p<0.01 ; * * * p<0.001$ 


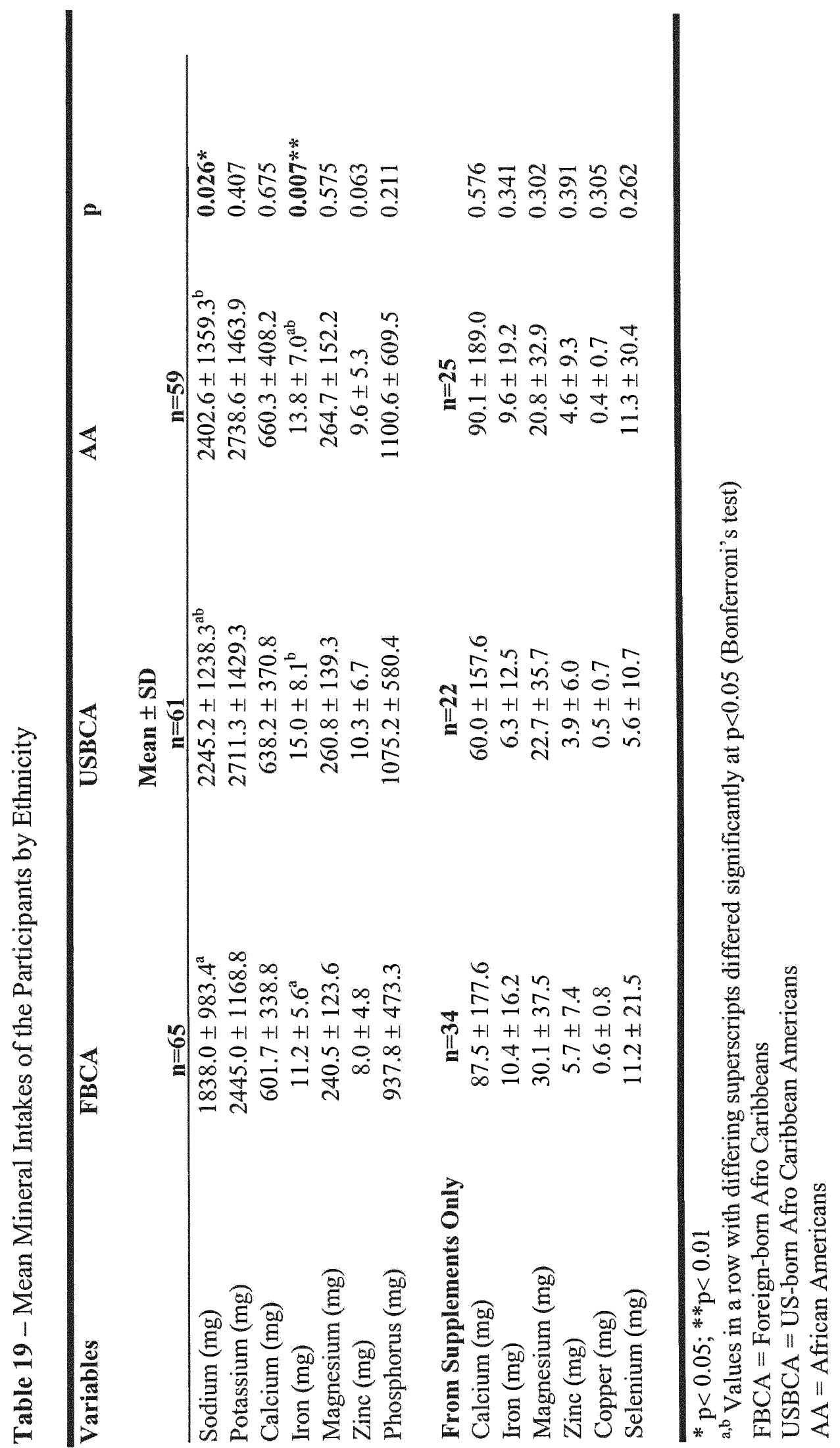




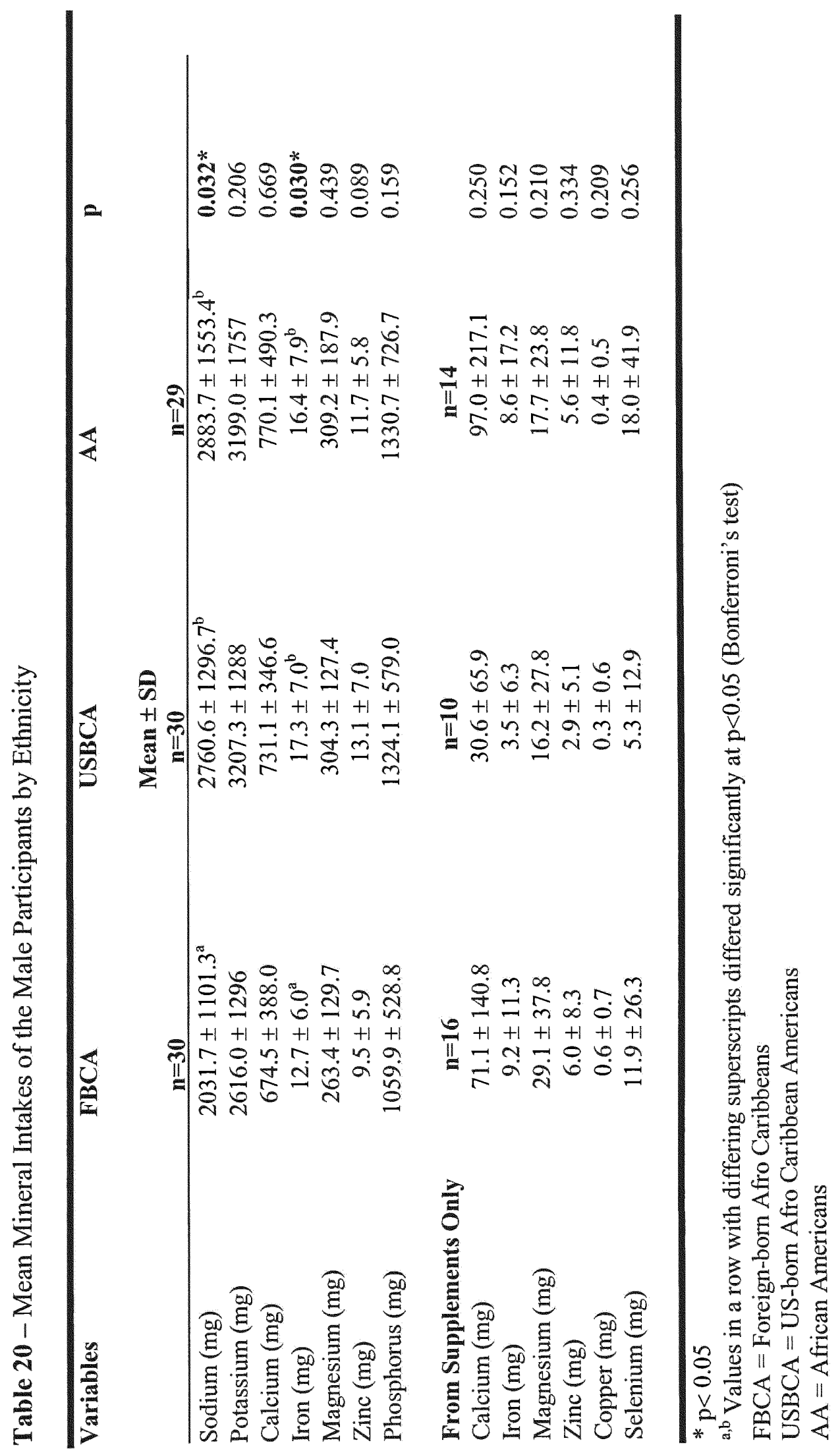




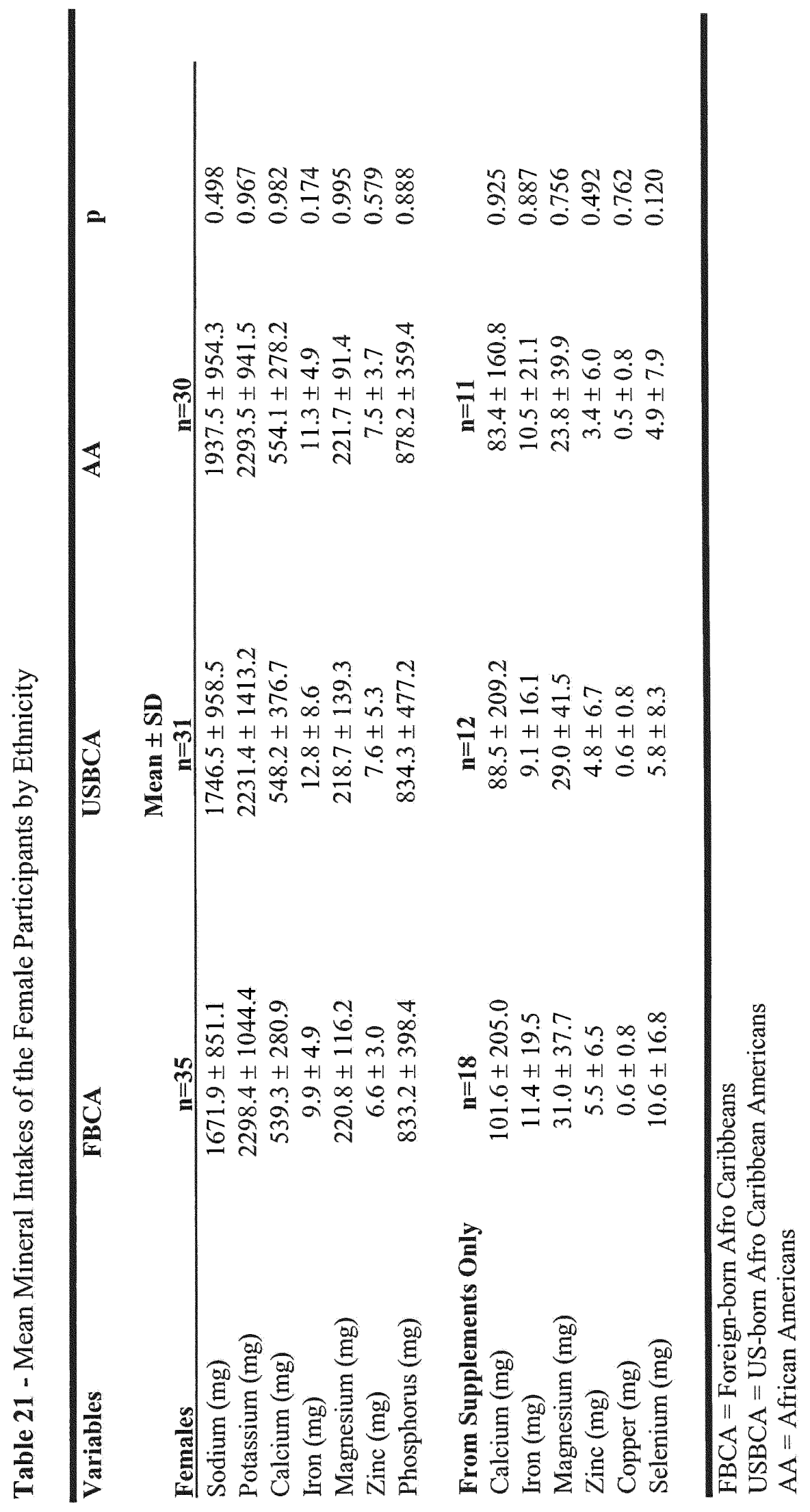


Males had significantly higher mean intakes of the energy-related vitamins such as vitamin $B 1(\mathrm{mg})$ (thiamin) (1.8 \pm 0.9 vs. $1.3 \pm 0.7 ; \mathrm{p}<0.001)$, vitamin $\mathrm{B} 2$ (mg) (riboflavin) (1.8 \pm 0.9 vs. $1.3 \pm 0.7 ; \mathrm{p}<0.001)$ and niacin $(\mathrm{mg})(22.7 \pm 12.3$ vs. $14.6 \pm$ $7.3 ; \mathrm{p}<0.001)$. Males also had significantly higher intakes of folate $(\mu \mathrm{g})(409.9 \pm 204.1$ vs. $332.0 \pm 189.1 ; \mathrm{p}<0.01)$, vitamin $\mathrm{B} 6(\mathrm{mg})(2.0 \pm 1.0$ vs. $1.4 \pm 0.8 ; \mathrm{p}<0.001)$ and vitamin B12 $(\mu \mathrm{g})(6.2 \pm 6.0$ vs. $2.9 \pm 2.5 ; \mathrm{p}<0.001)$. The mean intakes of retinol $(\mu \mathrm{g})$ (preformed vitamin A) (377.1 \pm 252.9 vs. $243.0 \pm 163.3 ; \mathrm{p}<0.001)$, vitamin $\mathrm{E}$ (mg) (7.9 \pm 5.3 vs. $6.3 \pm 4.9 ; p<0.05)$ and vitamin $D(I U) ~(207.0 \pm 210.3$ vs. $112.0 \pm 91.3 ; p<$ 0.001) were also significantly higher in the males compared to females. There were no significant differences between male and female groups with respect to vitamin intakes consumed from supplements (Table 22).

Within ethnic groups comparison showed the FBCA participants compared to the USBCA and AA participants had significantly lower mean intake of the energy-related vitamin niacin $(\mathrm{mg})(15.1 \pm 8.2$ vs. $20.6 \pm 11.3$ and $20.0 \pm 12.1 ; \mathrm{p}<0.01)$. However, the mean intakes from supplements were significantly higher for vitamin B1 (mg) (1.4 \pm 2.7 vs. $0.4 \pm 0.7$ and $0.7 \pm 1.7 ; \mathrm{p}<0.05)$, Vitamin B2 (mg) $(1.4 \pm 2.7$ vs. $0.4 \pm 0.7$ and $0.8 \pm$ 1.8; $\mathrm{p}<0.05)$, Niacin (mg) $(15.0 \pm 26.9$ vs. $5.0 \pm 8.1$ and $8.3 \pm 18.3 ; \mathrm{p}<0.05)$, Vitamin B6 (mg) $(1.1 \pm 1.5$ vs. $0.5 \pm 0.7$ and $0.6 \pm 1.2 ; \mathrm{p}<0.05)$, Vitamin $B 12(\mu \mathrm{g})(2.9 \pm 3.8$ vs. 1.4 \pm 2.2 and $1.7 \pm 3.1 ; \mathrm{p}<0.05)$ and Vitamin $\mathrm{E}(\mathrm{mg})(41.6 \pm 104.1$ vs. $10.0 \pm 19.8$ and 18.3 $\pm 51.2 ; \mathrm{p}<0.05$ ) in FBCA compared to USBCA but not AA participants (Table 23). 
Table 22 - Mean Vitamin Intakes of the Participants by Gender

\begin{tabular}{|c|c|c|c|}
\hline & Male & $\begin{array}{l}\text { Female } \\
\text { SD }\end{array}$ & $\mathbf{p}$ \\
\hline & $\mathrm{n}=89$ & $\mathrm{n}=96$ & \\
\hline Vitamin B1 (mg) & $1.8 \pm 0.9$ & $1.3 \pm 0.7$ & $0.0001 * * *$ \\
\hline Vitamin B2 (mg) & $1.8 \pm 0.9$ & $1.3 \pm 0.7$ & $0.0001 * * *$ \\
\hline Niacin (mg) & $22.7 \pm 12.3$ & $14.6 \pm 7.3$ & $0.0001^{* * *}$ \\
\hline Folate $(\mu \mathrm{g})$ & $409.9 \pm 204.1$ & $332.0 \pm 189.1$ & $0.008 * *$ \\
\hline Vitamin B6 (mg) & $2.0 \pm 1.0$ & $1.4 \pm 0.8$ & $0.0001 * * *$ \\
\hline Vitamin B12 $(\mu \mathrm{g})$ & $6.2 \pm 6.0$ & $2.9 \pm 2.5$ & $0.0001^{* * *}$ \\
\hline $\operatorname{Retinol}^{1}(\mu \mathrm{g})$ & $377.1 \pm 252.9$ & $243.0 \pm 163.3$ & $0.0001 * * *$ \\
\hline Vitamin $A(\mu g)$ & $1188.8 \pm 948.1$ & $975.4 \pm 856.4$ & 0.109 \\
\hline Vitamin C (mg) & $163.9 \pm 107.3$ & $147.8 \pm 102.6$ & 0.298 \\
\hline Vitamin $\mathrm{E}(\mathrm{mg})$ & $7.9 \pm 5.3$ & $6.3 \pm 4.9$ & $0.024^{*}$ \\
\hline Vitamin D (IU) & $207.0 \pm 210.3$ & $112.0 \pm 91.3$ & $0.0001^{* * *}$ \\
\hline From Supplements Only & $\mathrm{n}=40$ & $n=43$ & \\
\hline Vitamin B1 (mg) & $0.8 \pm 1.9$ & $0.9 \pm 2.0$ & 0.631 \\
\hline Vitamin B2 (mg) & $0.8 \pm 1.8$ & $1.0 \pm 2.1$ & 0.605 \\
\hline Niacin (mg) & $8.7 \pm 18.7$ & $10.4 \pm 21.1$ & 0.566 \\
\hline Folate $(\mu \mathrm{g})$ & $118.2 \pm 172.1$ & $144.7 \pm 208.1$ & 0.349 \\
\hline Vitamin B6 (mg) & $0.7 \pm 1.1$ & $0.8 \pm 1.3$ & 0.389 \\
\hline Vitamin B12 $(\mu \mathrm{g})$ & $1.8 \pm 2.9$ & $2.3 \pm 3.4$ & 0.323 \\
\hline Vitamin A $(\mu \mathrm{g})$ & $1372.7 \pm 2089.1$ & $1689.2 \pm 2422.1$ & 0.344 \\
\hline Vitamin C (mg) & $102.9 \pm 199.7$ & $113.6 \pm 216.3$ & 0.728 \\
\hline Vitamin E (mg) & $18.4 \pm 44.7$ & $28.7 \pm 87.2$ & 0.315 \\
\hline Vitamin D (IU) & $93.8 \pm 134.1$ & $118.2 \pm 164.4$ & 0.272 \\
\hline
\end{tabular}

$* \mathrm{p}<0.05 ; * * \mathrm{p}<0.01 ; * * * \mathrm{p}<0.001$

${ }^{1}$ Retinol $=$ preformed Vitamin $\mathrm{A}$ 


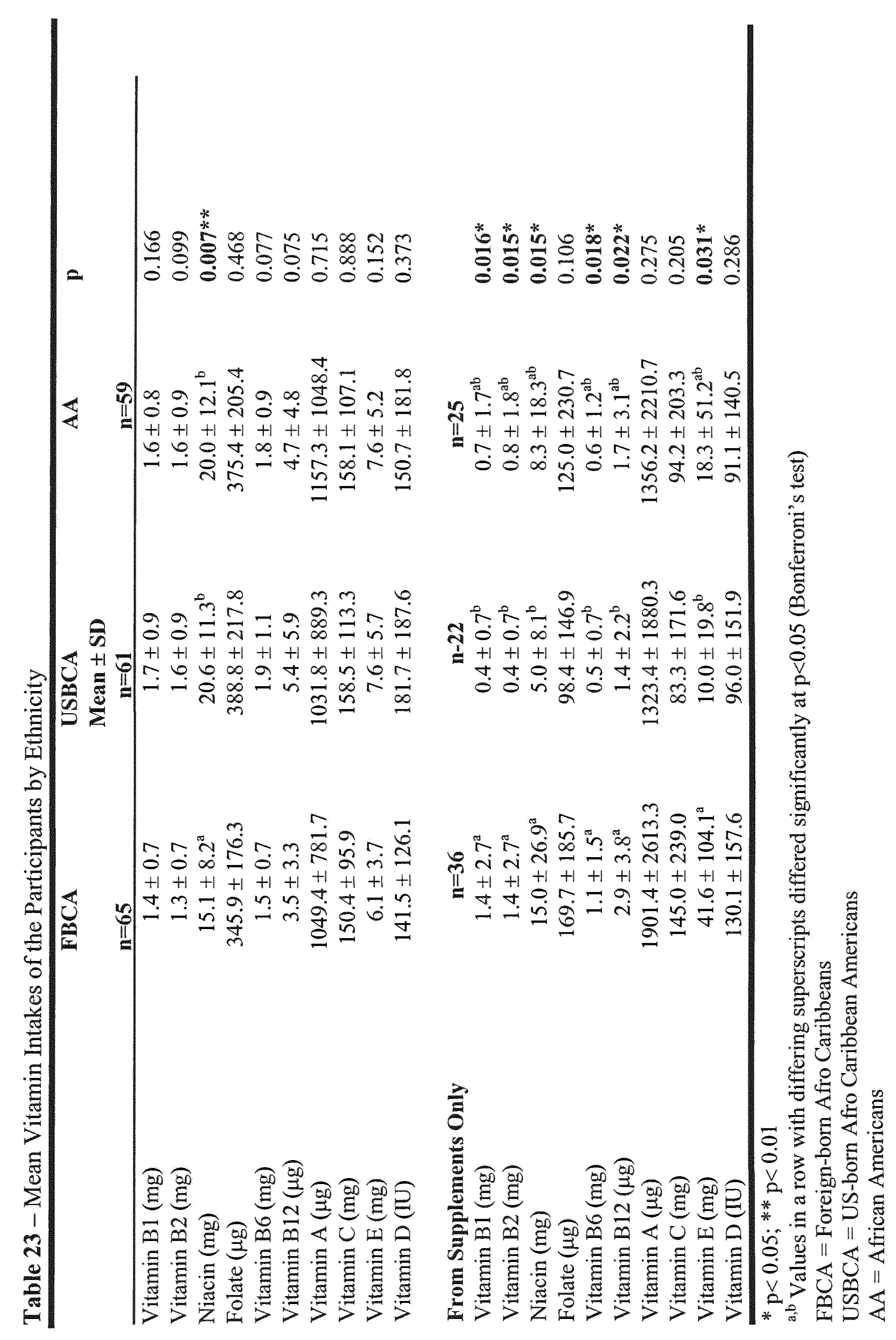


Within male ethnic groups comparison showed the mean niacin intake (mg) of the FBCA males $(17.5 \pm 9.2)$ was significantly $(\mathrm{p}<0.05)$ lower compared to the USBCA (26.0 \pm 11.4$)$ and AA males $(24.8 \pm 14.5)$. The mean vitamin intakes from supplements were significantly higher for niacin $(\mathrm{mg})(15.3 \pm 28.2$ vs. $4.2 \pm 7.9$ and $6.5 \pm 11.5$; $\mathrm{p}<$ $0.05)$, vitamin $B 6(\mathrm{mg})(1.1 \pm 1.6$ vs. $0.4 \pm 0.6$ and $0.5 \pm 0.8 ; \mathrm{p}<0.05)$ and vitamin $B 12$ $(\mu \mathrm{g})(2.9 \pm 4.1$ vs. $1.1 \pm 1.8$ and $1.4 \pm 2.1 ; \mathrm{p}<0.05)$ in FBCA males compared to the USBCA and AA males (Table 24).

There were no significant differences between the mean vitamin intakes or the mean vitamin intakes from supplements between the three female ethnic subgroups (Table 25).

\section{Dietary Intakes of Carotenoids}

The mean dietary intakes of carotenoids of the participants varied widely (Table 26). Within ethnic groups comparison showed the mean dietary intake of lycopene $(\mu \mathrm{g})$ was significantly $(p<0.01)$ lower in FBCA participants $(3431.7 \pm 3403.3)$ compared to USBCA (6038.2 \pm 6365.1$)$ and AA participants (5291.9 \pm 4227.3$)$ (Table 27).

Within male ethnic subgroups comparison showed the USBCA males compared to AA but not FBCA males had significantly $(\mathrm{p}<0.05)$ higher mean dietary intakes of daidzein $(\mu \mathrm{g})(559.1 \pm 1530.1$ vs. 0.0 and $85.6 \pm 346.7)$ and genisten $(\mu \mathrm{g})(807.5 \pm 2201.1$ vs. 0.0 and $123.6 \pm 500.8$ ), respectively (Table 28). 


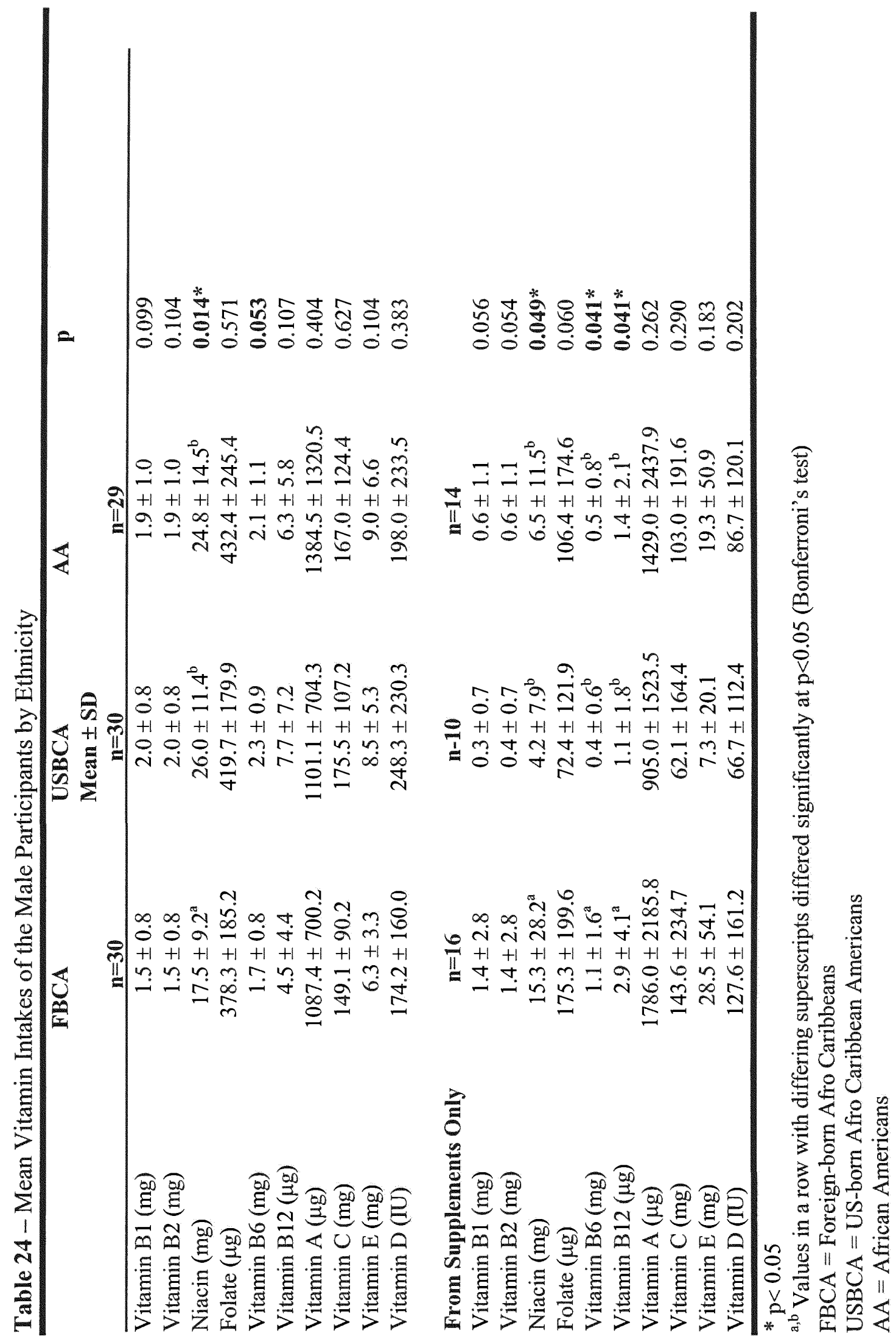




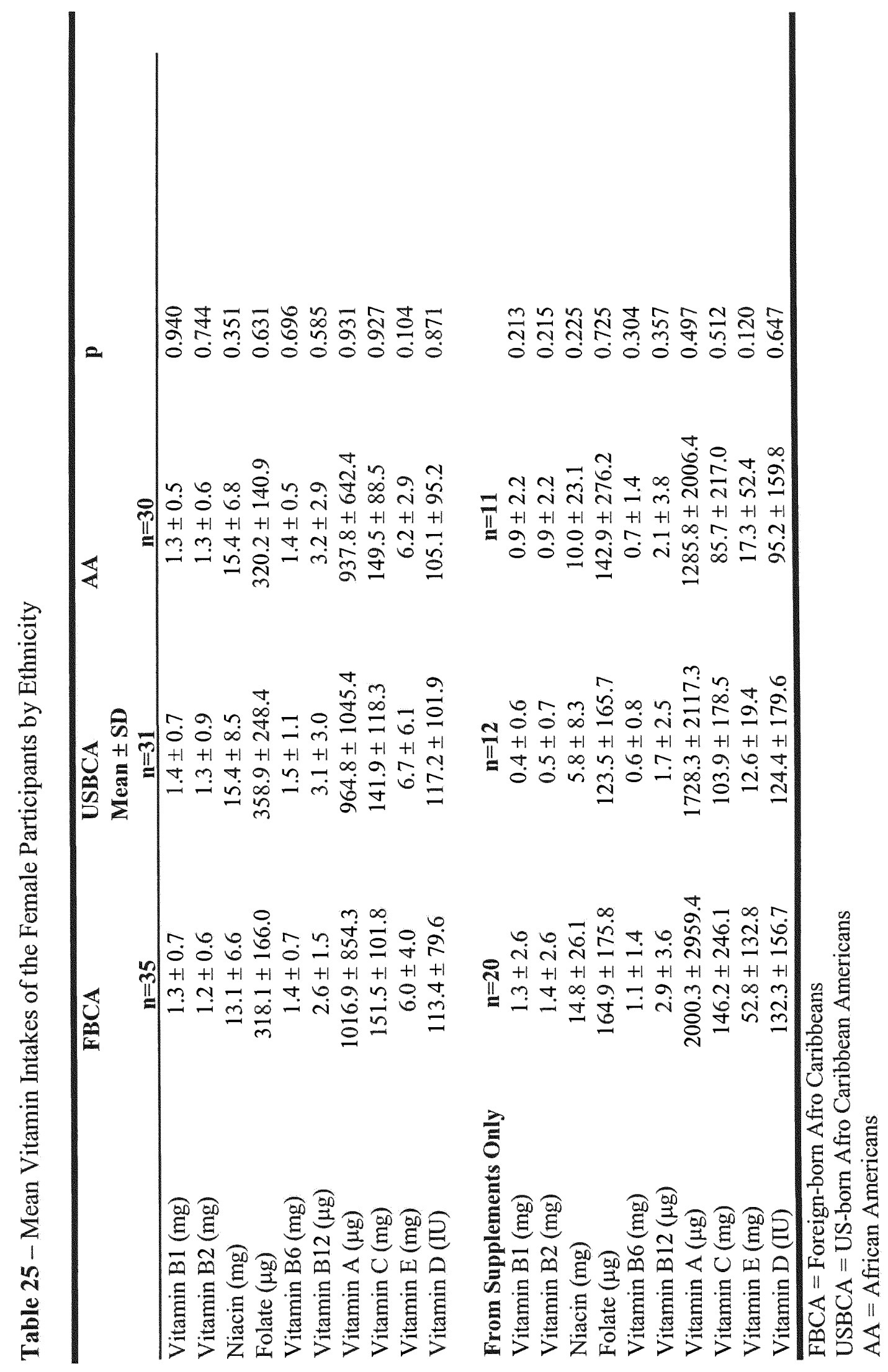


Table 26-Mean Carotenoid Intakes of the Participants by Gender

\begin{tabular}{|c|c|c|c|}
\hline \multirow[t]{2}{*}{ Variables } & Males & Females & $\mathbf{p}$ \\
\hline & \multicolumn{2}{|c|}{ Mean \pm SD } & \\
\hline Total Carotenes $^{1}(\mu \mathrm{g})$ & $4870.9 \pm 5174.6$ & $4396.0 \pm 4977.3$ & 0.525 \\
\hline$\alpha$-carotene $(\mu \mathrm{g})$ & $678.4 \pm 965.9$ & $610.9 \pm 873.8$ & 0.618 \\
\hline$\beta$-carotene $(\mu \mathrm{g})$ & $3679.0 \pm 3704.5$ & $3261.5 \pm 3570.8$ & 0.436 \\
\hline Cryptoxanthin $(\mu \mathrm{g})$ & $228.0 \pm 202.2$ & $232.0 \pm 199.8$ & 0.891 \\
\hline Lutein $(\mu \mathrm{g})$ & $1509.7 \pm 1652.9$ & $1740.4 \pm 2179.4$ & 0.421 \\
\hline \multirow[t]{2}{*}{ Lycopene $(\mu \mathrm{g})$} & $5582.3 \pm 5390.5$ & $4237.4 \pm 4347.8$ & 0.063 \\
\hline & $\mathrm{n}=7$ & $n=14$ & \\
\hline Daidzein $(\mu \mathrm{g})$ & $217.3 \pm 934.0$ & $505.7 \pm 1511.4$ & 0.124 \\
\hline Genisten $(\mu \mathrm{g})$ & $313.9 \pm 1349.2$ & $730.5 \pm 2183.2$ & 0.124 \\
\hline
\end{tabular}

${ }^{1}$ Carotene, Provitamin A corotenoids 


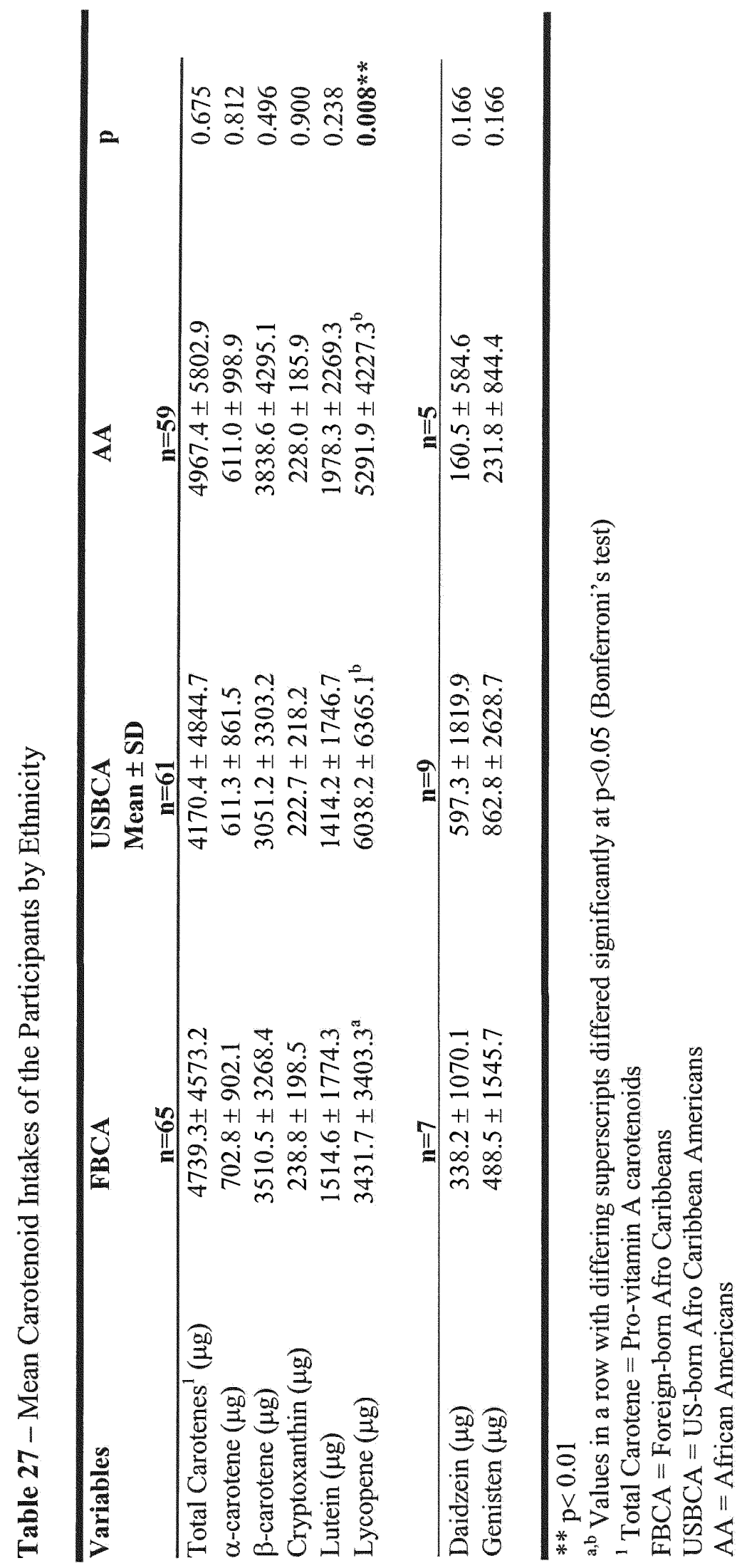




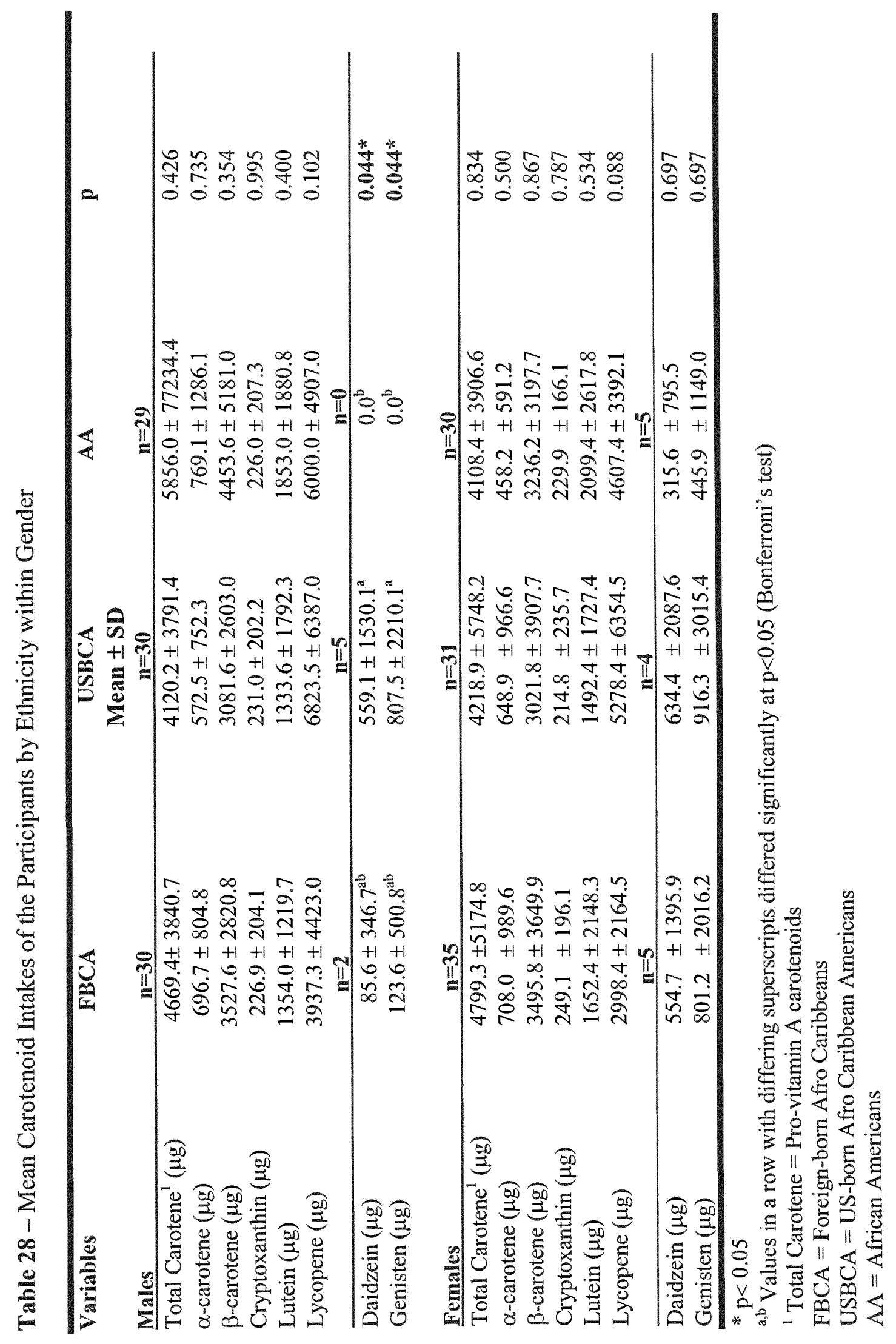




\section{Macronutrient Intakes Compared to NHANES III}

Males in this study, compared to non-Hispanic Black males in the NHANES III (1988-1994), had significantly $(\mathrm{p}<0.05)$ lower total fat $(78.6 \pm 48.2 \mathrm{~g}$ vs. $94.6 \mathrm{~g})$, saturated fat ( $27.3 \pm 16.4 \mathrm{~g}$ vs. $31.7 \mathrm{~g})$ and dietary cholesterol $(271.4 \pm 167.7 \mathrm{mg}$ vs. $358.8 \mathrm{mg}$ ) intakes (Table 29). Similarly for the females, the total fat $(49.8 \pm 28.0 \mathrm{~g}$ vs. $71.2 \mathrm{~g})$, saturated fat $(17.3 \pm 9.9 \mathrm{~g}$ vs. $23.8 \mathrm{~g})$ and dietary cholesterol $(152.8 \pm 95.0 \mathrm{mg}$ vs. $245.6 \mathrm{mg}$ ) intakes were significantly $(\mathrm{p}<0.05)$ lower than the NHANES III values, whereas the fiber intake $(14.2 \pm 10.2 \mathrm{~g}$ vs. $12.0 \mathrm{~g})$ was significantly $(\mathrm{p}<0.05)$ higher than the NHANES III value (Table 29).

Comparison by ethnic groups showed the FBCA and USBCA participants total fat $(52.8 \pm 32.3 \mathrm{~g}$ and $66.3 \pm 41.7 \mathrm{~g}$ vs. $82.0 \mathrm{~g})$, saturated fat $(18.6 \pm 11.5 \mathrm{~g}$ and $23.1 \pm 14.9 \mathrm{~g}$ vs. $27.5 \mathrm{~g})$ and dietary cholesterol (168.8 $\pm 114.0 \mathrm{mg}$ and $220.4 \pm 161.9 \mathrm{mg}$ vs. $297.9 \mathrm{mg})$ intakes were significantly $(\mathrm{p}<0.05)$ lower than the NHANES III values, whereas for the AA participants only dietary cholesterol $(244.1 \pm 155.0 \mathrm{mg}$ vs. $297.9 \mathrm{mg})$ intake was significantly $(p<0.05)$ lower than the NHANES III value (Table 29).

Only the FBCA males total fat $(59.2 \pm 37.4 \mathrm{~g}$ vs. $94.6 \mathrm{~g})$, saturated fat $(21.4 \pm$ $13.4 \mathrm{~g}$ vs. $31.7 \mathrm{~g})$ and dietary cholesterol $(211.2 \pm 134.1 \mathrm{mg}$ vs. $358.8 \mathrm{mg})$ intakes were significantly $(p<0.001)$ lower than the NHANES III values (Table 29).

For the FBCA and USBCA females, total fat $(47.3 \pm 26.5 \mathrm{~g}$ and $46.5 \pm 23.1 \mathrm{~g}$ vs. $71.2 \mathrm{~g})$, saturated fat $(16.3 \pm 9.2 \mathrm{~g}$ and $16.2 \pm 8.0 \mathrm{~g}$ vs. $23.9 \mathrm{~g})$ and dietary cholesterol $(132.6 \pm 78.6 \mathrm{mg}$ and $141.2 \pm 64.7 \mathrm{mg}$ vs. $245.6 \mathrm{mg})$ intakes were significantly ( $\mathrm{p}<$ 0.001) lower than the NHANES III values (Table 29). 
Table 29 - Macronutrient Intakes of the Participants Compared with NHANES $\operatorname{III}^{1}$ by Gender, Ethnicity and Gender-ethnicity Subgroups

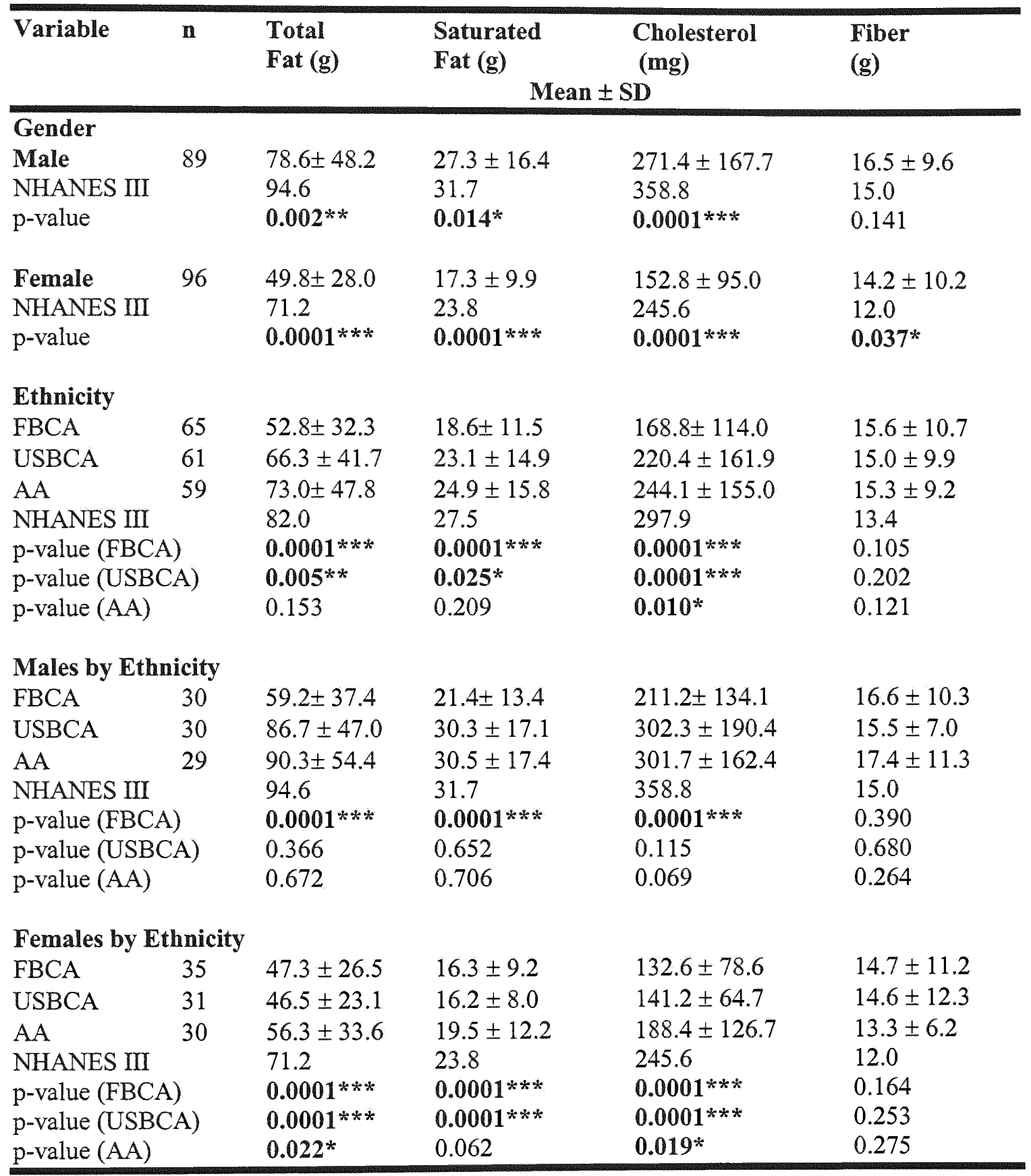

* $\mathrm{p}<0.05 ; * * \mathrm{p}<0.01 ; * * * \mathrm{p}<0.001$

${ }^{1}$ NHANES III (values are for non-Hispanic Blacks, Popkin et al., 2001)

FBCA = Foreign-born Afro Caribbeans;

USBCA = US-born Afro Caribbean Americans

$\mathrm{AA}=$ African Americans 
However, for the AA females, only total fat $(56.3 \pm 33.6 \mathrm{~g}$ vs. $71.2 \mathrm{~g})$ and dietary cholesterol (188.4 $\pm 126.7 \mathrm{mg}$ vs. $245.6 \mathrm{mg})$ intakes were significantly $(\mathrm{p}<0.05)$ lower than the NHANES III value (Table 29).

\section{Dietary Guidelines and Food Group Servings}

In comparing the participants reported dietary intakes with the American Heart Association/National Cholesterol Education Program Dietary Guidelines and the USDA Food Guide Pyramid serving guidelines, a tendency to over-consume sodium, energy from fat and, under-consume the food group servings, especially fruits, vegetables, grains and dairy was observed. More females compared to males, consumed significantly $(\mathrm{p}<$ $0.05)$ more energy from carbohydrate ( $50.0 \%$ vs. $31.5 \%)$, folate $(71.9 \%$ vs. $53.9 \%)$, vitamin B6 (43.8\% vs. $27.0 \%)$ and vitamin B12 (54.2\% vs. $23.6 \%)$, whereas more males consumed significantly $(\mathrm{p}<0.05)$ more energy from fat $(69.7 \%$ vs. $53.1 \%)$, saturated fat ( $56.2 \%$ vs. $32.3 \%)$, dietary cholesterol ( $38.2 \%$ vs. $7.3 \%)$ and sodium ( $48.3 \%$ vs. $20.8 \%)$ (Table 30).

More FBCA (55.4\%) participants than AA (25.4\%) but not the USBCA participants $(41.0 \%)$ consumed significantly $(\mathrm{p}<0.01)$ more energy from carbohydrate. In addition, more FBCA participants than USBCA and AA participants consumed significantly $(\mathrm{p}<0.01)$ less energy from fat $(46.2 \%$ vs. $65.6 \%$ and $72.9 \%)$. Also, more FBCA (21.5\%) participants compared to AA (5.1\%) but not USBCA (13.1\%) participants consumed significantly $(\mathrm{p}<0.01)$ more than two servings of fruits per day (Table 31$)$. 
Table 30 - Percentages of the Participants Consuming Below or Above the Dietary Guidelines $^{1}$ and Food Guide Pyramid Servings ${ }^{2}$ of Selected Macronutrients by Gender

\begin{tabular}{|c|c|c|c|c|}
\hline Variables & $\begin{array}{l}\text { Males } \\
\mathrm{n}=89 \\
\%\end{array}$ & $\begin{array}{l}\text { Females } \\
\mathrm{n}=96 \\
\%\end{array}$ & $\mathbf{p}$ & $\begin{array}{l}\text { Dietary } \\
\text { Guidelines }{ }^{1} \& \text { Food } \\
\text { Guide Sevings }{ }^{2}\end{array}$ \\
\hline \multicolumn{5}{|c|}{ Kcal Carbohydrate (\%) } \\
\hline$<55$ & 68.5 & 50.0 & $0.010^{*}$ & $55-60$ \\
\hline $\begin{array}{r}\geq 55 \\
\end{array}$ & 31.5 & 50.0 & & \\
\hline \multicolumn{5}{|l|}{ Kcal Fat (\%) } \\
\hline$<30$ & 30.3 & 46.9 & $0.021 *$ & $<30$ \\
\hline$\geq 30$ & 69.7 & 53.1 & & \\
\hline \multicolumn{5}{|c|}{ Saturated Fat (g) } \\
\hline$\leq 20$ & 43.8 & 67.7 & $0.001^{* *}$ & $\leq 20$ \\
\hline$>20$ & 56.2 & 32.3 & & \\
\hline
\end{tabular}

Cholesterol (mg)

$\begin{array}{llll}\leq 300 & 61.8 & 92.7 & \mathbf{0 . 0 0 0 1 * * *} \leq 300 \\ >300 & 38.2 & 7.3 & \end{array}$

Sodium (mg)

$\begin{array}{lllll}\leq 2400 & 51.7 & 79.2 & 0.0001^{* * *} & 2400 \\ >2400 & 48.3 & 20.8 & & \end{array}$

Fiber $(\mathrm{g})$

$\begin{array}{lllll}\geq 25 & 15.7 & 11.5 & 0.396 & 25-35 \\ <25 & 84.3 & 88.5 & & \end{array}$

Fruits (servings/d)

$\begin{array}{lllll}\geq 2 & 10.1 & 16.7 & 0.193 & 2-4 \\ <2 & 89.9 & 83.3 & & \end{array}$

Vegetables (servings/d)

$\begin{array}{lll}\geq 3 & 25.8 & 28.1 \\ <3 & 74.2 & 71.9\end{array}$

Folate $(\mu \mathrm{g})$

$\begin{array}{lllll}\geq 400 & 46.1 & 28.1 & \mathbf{0 . 0 1 1}^{*} & 400^{3} \\ <400 & 53.9 & 71.9 & & \end{array}$

Vitamin B6 (mg)

$\begin{array}{lllll}\geq 1.3 & 73.0 & 56.3 & 0.017^{*} & 1.3 \\ <1.3 & 27.0 & 43.8 & & \end{array}$

Vitamin B12 $(\mu \mathrm{g})$

$\begin{array}{lllll}\geq 2.4 & 76.4 & 45.8 & 0.0001 * * * & 2.4 \\ <2.4 & 23.6 & 54.2 & & \end{array}$

${ }^{*} \mathrm{p}<0.05 ; * * \mathrm{p}<0.01 ; * * * \mathrm{p}<0.001$

${ }^{1}$ Dietary Guidelines of Americans (AHA statistical Update, 2003; NCEP: ATP III, 2001)

${ }^{2}$ Food Guide Pyramid (www.usda.gov/fnic/Fpyr/pyramid.html. Accessed 05/16/04)

${ }^{3}$ As dietary folate equivalent (DFE) 
Table 31 - Percentages of the Participants Consuming Below or Above the Dietary Guidelines $^{1}$ and Food Guide Pyramid Servings ${ }^{2}$ of Selected Macronutrients by Ethnicity

\begin{tabular}{|c|c|c|c|c|c|}
\hline Variables & $\begin{array}{l}\text { FBCA } \\
n=65 \\
\% \\
\end{array}$ & $\begin{array}{l}\text { USBCA } \\
\mathrm{n}=61 \\
\%\end{array}$ & $\begin{array}{l}\text { AA } \\
\mathrm{n}=59 \\
\%\end{array}$ & $\mathrm{p}$ & $\begin{array}{r}\text { Dietary } \\
\text { Guidelines }{ }^{1} \& \\
\text { Food Guide } \\
\text { Sevings }\end{array}$ \\
\hline \multicolumn{6}{|c|}{ Kcal Carbohydrate (\%) } \\
\hline$<55$ & 44.6 & 59.0 & 74.6 & $0.003^{* *}$ & $55-60$ \\
\hline$\geq 55$ & $55.4^{\mathrm{a}}$ & $41.0^{\mathrm{ab}}$ & $25.4^{\mathrm{b}}$ & & \\
\hline \multicolumn{6}{|l|}{ Keal Fat (\%) } \\
\hline$<30$ & $53.8^{\mathrm{a}}$ & $34.4^{\mathrm{b}}$ & $27.1^{\mathrm{b}}$ & $0.007^{* *}$ & $<30$ \\
\hline$\geq 30$ & 46.2 & 65.6 & 72.9 & & \\
\hline \multicolumn{6}{|c|}{ Saturated Fat (g) } \\
\hline$\leq 20$ & 64.6 & 54.1 & 49.2 & 0.205 & $\leq 20$ \\
\hline$>20$ & 35.4 & 45.9 & 50.8 & & \\
\hline \multicolumn{6}{|c|}{ Cholesterol (mg) } \\
\hline$\leq 300$ & 83.1 & 77.0 & 72.9 & 0.387 & $\leq 300$ \\
\hline$>300$ & 16.9 & 23.0 & 27.1 & & \\
\hline \multicolumn{6}{|l|}{ Sodium (mg) } \\
\hline$\leq 2400$ & 73.8 & 62.3 & 61.0 & 0.246 & 2400 \\
\hline$>2400$ & 26.2 & 37.7 & 39.0 & & \\
\hline \multicolumn{6}{|l|}{ Fiber $(\mathrm{g})$} \\
\hline$\geq 25$ & 18.5 & 11.5 & 10.2 & 0.343 & $25-35$ \\
\hline$<25$ & 81.5 & 88.5 & 89.8 & & \\
\hline \multicolumn{6}{|c|}{ Fruits (servings/d) } \\
\hline$\geq 2$ & $21.5^{\mathrm{a}}$ & $13.1^{\mathrm{ab}}$ & $5.1^{\mathrm{b}}$ & $0.028^{*}$ & $2-4$ \\
\hline$<2$ & 78.5 & 86.9 & 94.9 & & \\
\hline \multicolumn{6}{|c|}{ Vegetables (servings/d) } \\
\hline$\geq 3$ & 30.8 & 19.7 & 30.5 & 0.287 & $3-5$ \\
\hline$<3$ & 69.2 & 80.3 & 69.5 & & \\
\hline \multicolumn{6}{|l|}{ Folate $(\mu \mathrm{g})$} \\
\hline$\geq 400$ & 32.3 & 41.0 & 37.3 & 0.598 & $400^{3}$ \\
\hline$<400$ & 67.7 & 59.0 & 62.7 & & \\
\hline \multicolumn{6}{|c|}{ Vitamin B6 (mg) } \\
\hline$\geq 1.3$ & 58.5 & 65.6 & 69.5 & 0.427 & 1.3 \\
\hline$<1.3$ & 41.5 & 34.4 & 30.5 & & \\
\hline \multicolumn{6}{|c|}{ Vitamin B12 $(\mu \mathrm{g})$} \\
\hline$\geq 2.4$ & 56.9 & 62.3 & 62.7 & 0.759 & 2.4 \\
\hline$<2.4$ & 43.1 & 37.7 & 37.3 & & \\
\hline
\end{tabular}

$* \mathrm{p}<0.05 ; * \mathrm{p}<0.01$

${ }^{a, b}$ Values in a row with differing superscripts differed significantly at $\mathrm{p}<0.05$ (Bonferroni's test)

${ }^{1}$ Dietary Guidelines of Americans (AHA statistical Update, 2003; NCEP: ATP III, 2001)

${ }^{2}$ Food Guide Pyramid (www.usda.gov/fnic/Fpyr/pyramid.html. Accessed 05/16/04)

${ }^{3}$ As dietary folate equivalent (DFE)

$\mathrm{FBCA}=$ Foreign-born Afro Caribbeans

USBCA = US-born Afro Caribbean Americans

$\mathrm{AA}=\mathrm{African}$ Americans 
Within male ethnic groups comparison showed significantly $(\mathrm{p}<0.05)$ more FBCA males (50.0\%) compared to AA (13.8\%) but not USBCA males $(30.0 \%)$, consumed more energy from carbohydrate, whereas significantly $(\mathrm{p}<0.05)$ more FBCA (50.0\%) males compared to AA (17.2\%) but not USBCA (23.3\%) males consumed less energy from fat (Table 32). With respect to the dietary guidelines, no significant differences between the three female ethnic subgroups were observed (Table 33).

\section{Diet Score}

A diet score was devised based on 7 selected recommended dietary behaviors (percent energy from fat, saturated fat, dietary cholesterol, fiber, sodium, fruit and vegetable servings). Individuals who exceeded the dietary recommendations for percent energy from fat, saturated fat, cholesterol and sodium were assigned a score of 0 and a score of 1 if they met the dietary requirements. Participants who consumed below the dietary recommendations for dietary fiber, fruit and vegetable servings were assigned a score of 0 and 1 if they met the dietary requirements. This score was summed across the selected food items to give a total diet score. The diet score ranged from $0-7$. Higher scores were indicative of good diet quality. For this study, the diet scores $0-2$ were categorized as 'poor diet quality', $3-5$ as 'moderate' and $\geq 6$ and greater as 'good' 
Table 32 - Percentages of Male Participants Consuming Below or Above the Dietary Guidelines $^{1}$ and Food Guide Pyramid Servings ${ }^{2}$ of Selected Macronutrients by Ethnicity

\begin{tabular}{|c|c|c|c|c|c|}
\hline Variables & $\begin{array}{l}\text { FBCA } \\
\mathrm{n}=30 \\
\%\end{array}$ & $\begin{array}{l}\text { USBCA } \\
\mathrm{n}=30 \\
\%\end{array}$ & $\begin{array}{l}\mathrm{AA} \\
\mathrm{n}=29 \\
\% \\
\end{array}$ & $\mathbf{p}$ & $\begin{array}{l}\text { Dietary } \\
\text { Guidelines }{ }^{1} \\
\text { \& Food Guide } \\
\text { Sevings }^{2} \\
\end{array}$ \\
\hline \multicolumn{6}{|l|}{$\mathrm{Kcal} \mathrm{CHO}^{3}(\%)$} \\
\hline$<55$ & 50.0 & 70.0 & 86.2 & $0.011^{*}$ & $55-60$ \\
\hline$\geq 55$ & $50.0^{\mathrm{a}}$ & $30.0^{\mathrm{ab}}$ & $13.8^{\mathrm{b}}$ & & \\
\hline \multicolumn{6}{|l|}{ Kcal Fat (\%) } \\
\hline$<30$ & 50.0 & 23.3 & 17.2 & $0.014^{*}$ & $<30$ \\
\hline$\geq 30$ & $50.0^{\mathrm{a}}$ & $76.7^{\mathrm{ab}}$ & $82.8^{b}$ & & \\
\hline \multicolumn{6}{|l|}{ Saturated Fat $(g)$} \\
\hline$\leq 20$ & 53.3 & 40.0 & 37.9 & 0.430 & $\leq 20$ \\
\hline$>20$ & 46.7 & 60.0 & 62.1 & & \\
\hline \multicolumn{6}{|l|}{ Cholesterol (mg) } \\
\hline$\leq 300$ & 70.0 & 56.7 & 58.6 & 0.519 & $\leq 300$ \\
\hline$>300$ & 30.0 & 43.3 & 41.4 & & \\
\hline \multicolumn{6}{|l|}{ Sodium (mg) } \\
\hline$\leq 2400$ & 66.7 & 46.7 & 41.4 & 0.120 & 2400 \\
\hline$>2400$ & 33.3 & 53.3 & 58.6 & & \\
\hline \multicolumn{6}{|l|}{ Fiber $(\mathrm{g})$} \\
\hline$\geq 25$ & 20.0 & 10.0 & 17.2 & 0.547 & $25-35$ \\
\hline$<25$ & 80.0 & 90.0 & 82.8 & & \\
\hline \multicolumn{6}{|c|}{ Fruits (servings/d) } \\
\hline$\geq 2$ & 20.0 & 6.7 & 3.4 & 0.081 & $2-4$ \\
\hline$<2$ & 80.0 & 93.3 & 96.6 & & \\
\hline \multicolumn{6}{|c|}{ Vegetables (servings/d) } \\
\hline$\geq 3$ & 23.3 & 16.7 & 37.9 & 0.163 & $3-5$ \\
\hline$<3$ & 76.7 & 83.3 & 62.1 & & \\
\hline \multicolumn{6}{|l|}{ Folate $(\mu \mathrm{g})$} \\
\hline$\geq 400$ & 43.3 & 43.3 & 51.7 & 0.758 & $400^{3}$ \\
\hline$<400$ & 56.7 & 56.7 & 48.3 & & \\
\hline \multicolumn{6}{|c|}{ Vitamin B6 (mg) } \\
\hline$\geq 1.3$ & 66.7 & 76.7 & 75.9 & 0.626 & 1.3 \\
\hline$<1.3$ & 33.3 & 23.3 & 24.1 & & \\
\hline \multicolumn{6}{|c|}{ Vitamin B12 $(\mu \mathrm{g})$} \\
\hline$\geq 2.4$ & 66.7 & 80.0 & 82.8 & 0.295 & 2.4 \\
\hline$<2.4$ & 33.3 & 20.0 & 17.2 & & \\
\hline
\end{tabular}

* $\mathrm{p}<0.05$

${ }^{a, b}$ Values in a row with differing superscripts differed significantly at $\mathrm{p}<0.05$ (Bonferroni's test)

${ }^{1}$ Dietary Guidelines of Americans (AHA statistical Update, 2003; NCEP: ATP III, 2001)

${ }^{2}$ Food Guide Pyramid (www.usda.gov/fnic/Fpyr/pyramid.html. Accessed 05/16/04)

${ }^{3}$ As dietary folate equivalent (DFE)

FBCA $=$ Foreign-born Afro Caribbeans

USBCA = US-born Afro Caribbean Americans

$\mathrm{AA}=$ African Americans 
Table 33 - Percentages of Female Participants Consuming Below or Above the Dietary Guidelines ${ }^{1}$ and Food Guide Pyramid Servings ${ }^{2}$ of Selected Macronutrients by Ethnicity

\begin{tabular}{|c|c|c|c|c|c|}
\hline Variables & $\begin{array}{l}\text { FBCA } \\
n=30 \\
\%\end{array}$ & $\begin{array}{l}\text { USBCA } \\
n=30 \\
\%\end{array}$ & $\begin{array}{l}\mathrm{AA} \\
\mathrm{n}=29 \\
\% \\
\end{array}$ & $\mathbf{p}$ & $\begin{array}{l}\text { Dietary } \\
\text { Guidelines } \\
{ }^{1} \\
\text { \& Food Guide } \\
\text { Sevings }^{2} \\
\end{array}$ \\
\hline \multicolumn{6}{|l|}{$\mathrm{Kcal} \mathrm{CHO}^{3}(\%)$} \\
\hline$<55$ & 40.0 & 48.4 & 63.3 & 0.168 & $55-60$ \\
\hline$\geq 55$ & 60.0 & 51.6 & 36.7 & & \\
\hline \multicolumn{6}{|l|}{ Kcal Fat (\%) } \\
\hline$<30$ & 57.1 & 45.2 & 36.7 & 0.250 & $<30$ \\
\hline$\geq 30$ & 42.9 & 54.8 & 63.3 & & \\
\hline \multicolumn{6}{|l|}{ Saturated Fat } \\
\hline$\leq 20$ & 74.3 & 67.7 & 60.0 & 0.471 & $\leq 20$ \\
\hline$>20$ & 25.7 & 32.3 & 40.0 & & \\
\hline \multicolumn{6}{|l|}{ Cholesterol (mg) } \\
\hline$\leq 300$ & 94.3 & 96.8 & 86.7 & 0.286 & $\leq 300$ \\
\hline$>300$ & 5.7 & 3.2 & 13.3 & & \\
\hline \multicolumn{6}{|l|}{ Sodium (mg) } \\
\hline$\leq 2400$ & 80.0 & 77.4 & 80.0 & 0.959 & 2400 \\
\hline$>2400$ & 20.0 & 22.6 & 20.0 & & \\
\hline \multicolumn{6}{|l|}{ Fiber $(g)$} \\
\hline$\geq 25$ & 17.1 & 12.9 & 3.3 & 0.209 & $25-35$ \\
\hline$<25$ & 82.9 & 87.1 & 96.7 & & \\
\hline \multicolumn{6}{|c|}{ Fruits (servings/d) } \\
\hline$\geq 2$ & 22.9 & 19.4 & 6.7 & 0.193 & $2-4$ \\
\hline$<2$ & 77.1 & 80.6 & 93.3 & & \\
\hline \multicolumn{6}{|c|}{ Vegetables (servings/d) } \\
\hline$\geq 3$ & 37.1 & 22.6 & 23.3 & 0.330 & $3-5$ \\
\hline$<3$ & 62.9 & 77.4 & 76.7 & & \\
\hline \multicolumn{6}{|l|}{ Folate $(\mu \mathrm{g})$} \\
\hline$\geq 400$ & 22.9 & 38.7 & 23.3 & 0.281 & $400^{3}$ \\
\hline$<400$ & 77.1 & 61.3 & 76.7 & & \\
\hline \multicolumn{6}{|c|}{ Vitamin B6 (mg) } \\
\hline$\geq 1.3$ & 51.4 & 54.8 & 63.3 & 0.617 & 1.3 \\
\hline$<1.3$ & 48.6 & 45.2 & 36.7 & & \\
\hline \multicolumn{6}{|c|}{ Vitamin B12 $(\mu \mathrm{g})$} \\
\hline$\geq 2.4$ & 48.6 & 45.2 & 43.3 & 0.911 & 2.4 \\
\hline$<2.4$ & 51.4 & 54.8 & 56.7 & & \\
\hline
\end{tabular}

${ }^{1}$ Dietary Guidelines of Americans (AHA statistical Update, 2003; NCEP: ATP III, 2001)

${ }^{2}$ Food Guide Pyramid (www.usda.gov/fnic/Fpyr/pyramid.html. Accessed 05/16/04)

${ }^{3} \mathrm{CHO}=$ Carbohydrate

FBCA $=$ Foreign-born Afro Caribbeans

USBCA = US-born Afro Caribbean Americans

$\mathrm{AA}=$ African Americans 
The mean diet score of males $(2.4 \pm 1.7)$ was significantly $(\mathrm{p}<0.001)$ lower than females $(3.4 \pm 1.4)$. Also, the percentage of males (32.6\%) with diet scores $0-2$ was significantly $(\mathrm{p}<0.001)$ higher than females $(8.3 \%)$ (Table 34$)$.

Within ethnic groups comparison showed the mean diet score of the FBCA participants $(3.5 \pm 1.6)$ was significantly $(\mathrm{p}<0.01)$ higher than the USBCA $(2.7 \pm 1.7)$ and AA participants (2.6 \pm 1.5$)$ (Table 34). Within male ethnic subgroups comparison showed similar trends as the mean diet score of FBCA males $(3.0 \pm 1.6)$ was significantly $(\mathrm{p}<0.05)$ higher than the USBCA $(2.0 \pm 1.9)$ but not the AA males $(2.1 \pm 1.4)$. Also, the percentage of FBCA males $(16.7 \%)$ with diet score $0-2$ was significantly $(\mathrm{p}<0.05)$ lower than USBCA (50.0\%) but not AA males (31.0\%) (Table 34). However, the mean diet score of FBCA females $(3.8 \pm 1.5)$ was significantly $(\mathrm{p}<0.05)$ higher compared to the AA (3.0 \pm 1.5$)$ but not the USBCA females $(3.4 \pm 0.9)$ (Table 34).

\section{Physical Activity Indices Scores, Sports, and Television Watching}

The work activity index (WAI), sports activity index (SAI) and leisure time activity index (LTAI) scores ranged from $0.0-5.0$. The summation of these scores is named the physical activity index (PAI) score. The PAI score ranged from 0.0-15.0. Although more males than females had significantly $(\mathrm{p}<0.001)$ higher mean PAI $(8.4 \pm$ 1.1 vs. $7.2 \pm 1.3)$, WAI ( $2.7 \pm 0.7$ vs. $2.4 \pm 0.5)$, SAI ( $3.0 \pm 0.8$ vs. $2.2 \pm 0.8)$ and played sports ( $73.6 \%$ vs. $26.5 \%$ ), no significant differences were observed between the three ethnic groups, the three male ethnic subgroups or the three female ethnic subgroups with respect to these variables (Tables 35 and 36). 
Table 34 - Mean and Frequency Distribution of the Diet Scores of Participants by Gender, Ethnicity and Gender-ethnicity Subgroups

\begin{tabular}{|c|c|c|c|c|c|}
\hline \multirow[t]{2}{*}{ Variable } & \multirow[b]{2}{*}{$\mathbf{n}$} & \multirow{2}{*}{$\begin{array}{l}\text { Diet Score } \\
\text { Mean } \pm \text { SD }\end{array}$} & \multicolumn{3}{|c|}{ Diet Score Categories } \\
\hline & & & $\begin{array}{l}0-2 \\
\%\end{array}$ & $\begin{array}{l}3-5 \\
\%\end{array}$ & $\begin{array}{l}\geq 6 \\
\%\end{array}$ \\
\hline \multicolumn{6}{|l|}{ Gender } \\
\hline Male & 89 & $2.4 \pm 1.7$ & 32.6 & 56.2 & 11.2 \\
\hline Female & 96 & $3.4 \pm 1.4$ & 8.3 & 75.0 & 16.7 \\
\hline $\mathrm{p}$-value & & $0.001 * * *$ & & $0.001 * * *$ & \\
\hline \multicolumn{6}{|l|}{ Ethnicity } \\
\hline FBCA & 65 & $3.5 \pm 1.6^{\mathrm{a}}$ & 10.8 & 69.2 & 20.0 \\
\hline USBCA & 61 & $2.7 \pm 1.7^{b}$ & 26.2 & 62.3 & 11.5 \\
\hline $\mathrm{AA}$ & 59 & $2.6 \pm 1.5^{b}$ & 23.7 & 66.1 & 10.2 \\
\hline $\mathrm{p}$-value & & $0.003^{* *}$ & & 0.128 & \\
\hline \multicolumn{6}{|c|}{ Males by Ethnicity } \\
\hline FBCA & 30 & $3.0 \pm 1.6^{\mathrm{a}}$ & $16.7^{\mathrm{a}}$ & 66.7 & 16.7 \\
\hline USBCA & 30 & $2.0 \pm 1.9^{\mathrm{b}}$ & $50.0^{b}$ & 36.7 & 13.3 \\
\hline $\mathrm{AA}$ & 29 & $2.1 \pm 1.4^{\mathrm{ab}}$ & $31.0^{\mathrm{ab}}$ & 65.5 & 3.4 \\
\hline $\mathrm{p}$-value & & $0.038^{*}$ & & $0.030^{*}$ & \\
\hline \multicolumn{6}{|c|}{ Females by Ethnicity } \\
\hline FBCA & 35 & $3.8 \pm 1.5^{\mathrm{a}}$ & 3.2 & 87.1 & 9.7 \\
\hline USBCA & 31 & $3.4 \pm 0.9^{\mathrm{ab}}$ & 3.2 & 87.1 & 9.7 \\
\hline $\mathrm{AA}$ & 30 & $3.0 \pm 1.5^{b}$ & 16.7 & 66.7 & 16.7 \\
\hline $\mathrm{p}$-value & & $0.036^{*}$ & & 0.170 & \\
\hline
\end{tabular}

$* \mathrm{p}<0.05 ; * * \mathrm{p}<0.01 ; * * * \mathrm{p}<0.001$

${ }^{a, b}$ Values in a column with differing superscripts differed significantly at $\mathrm{p}<0.05$ (Bonferroni's test)

Diet Score Range $0-7$, where 0 is poor diet and 7 good diet

FBCA $=$ Foreign-born Afro Caribbeans

USBCA = US-born Afro Caribbean Americans

$\mathrm{AA}=$ African Americans 
Table 35 - Mean Physical Activity Indices of the Participants by Gender, Ethnicity and Gender-ethnicity Subgroups



$* * * \mathrm{p}<0.001$

$\mathrm{PAI}=$ Physical Activity Index, Range 3.0 - 15.0, where 3.0 is low and 15.0 is high; WAI = Work Activity Index, Range $1.0-5.0$, where 1.0 is low and 5.0 is high; SAI = Sports Activity Index, Range 1.0-5.0, where 1.0 is low and 5.0 is high; LTAI = Leisure Time Activity Index, Range $1.0-5.0$, where 1.0 is low and 5.0 is high

FBCA = Foreign-born Afro Caribbeans

USBCA = US-born Afro Caribbean Americans

$\mathrm{AA}=$ African Americans 
Table 36 - Distribution of the Physical Activities of the Participants by Gender, Ethnicity and Gender-ethnicity Subgroups

\begin{tabular}{|c|c|c|c|c|c|}
\hline \multirow[t]{2}{*}{ Variable } & \multirow[t]{2}{*}{$\mathbf{n}$} & \multicolumn{2}{|c|}{ Played Sports } & \multicolumn{2}{|c|}{ Watched Television } \\
\hline & & No & Yes & $\begin{array}{l}\text { Never/ } \\
\text { Sometimes }\end{array}$ & $\begin{array}{l}\text { Often/ } \\
\text { Very Often }\end{array}$ \\
\hline \multicolumn{6}{|l|}{ Gender } \\
\hline Male & 91 & 26.4 & 73.6 & 43.7 & 52.7 \\
\hline Female & 98 & 73.5 & 26.5 & 53.1 & 46.9 \\
\hline $\mathrm{p}$-value & & \multicolumn{2}{|c|}{$0.001^{* * *}$} & \multicolumn{2}{|c|}{0.425} \\
\hline \multicolumn{6}{|l|}{ Ethnicity } \\
\hline FBCA & 66 & 40.9 & 59.1 & 54.5 & 45.5 \\
\hline USBCA & 62 & 56.5 & 43.5 & 45.2 & 54.8 \\
\hline $\mathrm{AA}$ & 61 & 55.7 & 44.3 & 50.8 & 49.2 \\
\hline p-value & & \multicolumn{2}{|c|}{0.137} & \multicolumn{2}{|c|}{0.566} \\
\hline \multicolumn{6}{|c|}{ Males by Ethnicity } \\
\hline FBCA & 31 & 16.1 & 83.9 & 58.1 & 41.9 \\
\hline USBCA & 30 & 30.0 & 70.0 & 40.0 & 60.0 \\
\hline $\mathrm{AA}$ & 30 & 33.3 & 66.7 & 43.3 & 56.7 \\
\hline $\mathrm{p}$-value & & \multicolumn{2}{|c|}{0.269} & \multicolumn{2}{|c|}{0.321} \\
\hline \multicolumn{6}{|c|}{ Females by Ethnicity } \\
\hline FBCA & 35 & 62.9 & 37.1 & 51.4 & 48.6 \\
\hline USBCA & 32 & 81.3 & 18.7 & 50.0 & 50.0 \\
\hline $\mathrm{AA}$ & 31 & 77.4 & 22.6 & 58.1 & 41.9 \\
\hline p-value & & \multicolumn{2}{|c|}{0.196} & \multicolumn{2}{|c|}{0.791} \\
\hline
\end{tabular}

$* * * \mathrm{p}<0.001$

FBCA $=$ Foreign-born Afro Caribbeans

USBCA = US-born Afro Caribbean Americans

$\mathrm{AA}=$ African Americans 


\section{Anthropometrics}

Body mass index [BMI; body weight $(\mathrm{kg}) /$ height $\left.\left(\mathrm{m}^{2}\right)\right]$ was used as an indicator of overall adiposity. Normal weight is defined as BMI $18.5-24.9 \mathrm{~kg} / \mathrm{m}^{2}$, overweight as BMI $25-29.9 \mathrm{~kg} / \mathrm{m}^{2}$ and obesity as BMI $\geq 30 \mathrm{~kg} / \mathrm{m}^{2}$ (Obesity: Class I BMI 30.0-34.9 $\mathrm{kg} / \mathrm{m}^{2}$; Class II BMI $35.0-39.9 \mathrm{~kg} / \mathrm{m}^{2}$; Class III BMI $40.0 \mathrm{~kg} / \mathrm{m}^{2}$ ). Abdominal obesity was defined as waist circumference (WC) greater than 40 inches for males and 35 inches for females (NCEP: ATP III, 2001).

The mean body weight $(85.1 \pm 21.0 \mathrm{~kg}$ vs. $66.1 \pm 13.6 \mathrm{~kg})$, height $(1.8 \pm 0.1 \mathrm{~m}$ vs. $1.6 \pm 0.1)$, BMI ( $26.9 \pm 5.8$ vs. $24.9 \pm 4.4)$ and $\mathrm{WC}(34.8 \pm 4.7$ vs. $31.6 \pm 4.0)$ were significantly $(\mathrm{p}<0.001)$ higher in males than females (Table 37). Also, significantly $(\mathrm{p}<$ $0.05)$ more males than females were overweight ( $36.3 \%$ vs. $26.5 \%)$ and obese $(19.8 \%$ vs. $12.2 \%$ ) (Table 38). Ethnic groups comparison showed that although there were no significant differences between the three ethnic groups with respect to the mean weight, height, BMI and WC values (Table 37), significantly $(\mathrm{p}<0.05)$ more AA $(23.0 \%)$ and USBCA participants $(17.7 \%)$ were obese compared to the FBCA participants $(7.6 \%)$ (Table 38). Similarly, although there were no significant differences between the three male ethnic subgroups with respect to the mean weight, height, BMI and WC values (Table 37), significantly $(\mathrm{p}<0.01)$ more AA $(33.3 \%)$ and USBCA males $(20.0 \%)$ were obese compared to the FBCA males (6.5\%) (Table 38). There were no significant differences between the three female ethnic subgroups with respect to the mean weight, height, BMI and WC values (Table 37) or the percentages of those who were obese and had abdominal obesity (Table 38). 
Table 37 - Anthropometrics of the Participants by Gender, Ethnicity and Genderethnicity Subgroups

\begin{tabular}{|c|c|c|c|c|c|}
\hline \multirow[t]{2}{*}{ Variable } & \multirow[t]{2}{*}{ n } & $\begin{array}{l}\text { Height } \\
\text { (m) }\end{array}$ & $\begin{array}{l}\text { Weight } \\
\text { (kg) }\end{array}$ & $\begin{array}{l}\text { BMI } \\
\left(\mathrm{kg} / \mathrm{m}^{2}\right)\end{array}$ & \multirow[t]{2}{*}{$\begin{array}{l}\text { WC } \\
\text { (inches) }\end{array}$} \\
\hline & & \multicolumn{3}{|c|}{ Mean \pm SD } & \\
\hline \multicolumn{6}{|l|}{ Gender } \\
\hline Male & 91 & $1.8 \pm 0.1$ & $85.1 \pm 21.0$ & $26.9 \pm 5.8$ & $34.8 \pm 4.7$ \\
\hline Female & 98 & $1.6 \pm 0.1$ & $66.1 \pm 13.6$ & $24.9 \pm 4.4$ & $31.6 \pm 4.0$ \\
\hline p-value & & $0.001 * * *$ & $0.001 * * *$ & $0.008 * *$ & $0.001 * * *$ \\
\hline \multicolumn{6}{|l|}{ Ethnicity } \\
\hline FBCA & 66 & $1.7 \pm 0.1$ & $71.7 \pm 15.1$ & $24.8 \pm 4.0$ & $32.6 \pm 4.1$ \\
\hline USBCA & 62 & $1.7 \pm 0.1$ & $76.1 \pm 17.9$ & $26.2 \pm 4.8$ & $33.5 \pm 4.6$ \\
\hline $\mathrm{AA}$ & 61 & $1.7 \pm 1.0$ & $78.4 \pm 25.4$ & $26.7 \pm 6.6$ & $33.5 \pm 5.2$ \\
\hline p-value & & 0.740 & 0.144 & 0.104 & 0.430 \\
\hline \multicolumn{6}{|c|}{ Males by Ethnicity } \\
\hline FBCA & 31 & $1.8 \pm 0.1$ & $80.6 \pm 14.1$ & $25.4 \pm 4.2$ & $33.8 \pm 3.9$ \\
\hline USBCA & 30 & $1.8 \pm 0.1$ & $85.4 \pm 16.9$ & $27.3 \pm 4.9$ & $35.6 \pm 4.2$ \\
\hline $\mathrm{AA}$ & 30 & $1.8 \pm 0.1$ & $89.5 \pm 28.9$ & $28.2 \pm 7.7$ & $35.0 \pm 5.8$ \\
\hline p-value & & 0.735 & 0.258 & 0.165 & 0.346 \\
\hline \multicolumn{6}{|c|}{ Females by Ethnicity } \\
\hline FBCA & 35 & $1.6 \pm 0.1$ & $63.6 \pm 11.1$ & $24.3 \pm 3.8$ & $31.5 \pm 4.0$ \\
\hline USBCA & 32 & $1.6 \pm 0.1$ & $67.3 \pm 14.2$ & $25.2 \pm 4.5$ & $31.6 \pm 4.0$ \\
\hline $\mathrm{AA}$ & 31 & $1.6 \pm 0.1$ & $67.7 \pm 15.6$ & $25.3 \pm 5.0$ & $32.1 \pm 4.1$ \\
\hline p-value & & 0.593 & 0.400 & 0.601 & 0.826 \\
\hline
\end{tabular}

** $\mathrm{p}<0.01 ; * * * \mathrm{p}<0.001$

$\mathrm{BMI}=$ Body Mass Index; $\mathrm{WC}=$ Waist Circumference

$\mathrm{FBCA}=$ Foreign-born Afro Caribbeans

USBCA $=$ US-born Afro Caribbean Americans

$\mathrm{AA}=$ African Americans 
Table 38 - Distribution of the Anthropometrics of the Participants by Gender, Ethnicity and Gender-ethnicity Subgroups

\begin{tabular}{|c|c|c|c|c|c|c|}
\hline \multirow[t]{2}{*}{ Variable } & \multirow[t]{2}{*}{$\mathbf{n}$} & \multicolumn{3}{|c|}{ BMI (kg/m2) } & \multicolumn{2}{|c|}{ WC (inches) } \\
\hline & & $\begin{array}{l}<25.0 \\
\% \\
\end{array}$ & $\begin{array}{l}25.0-29.9 \\
\% \\
\end{array}$ & $\begin{array}{c}\geq 30.0 \\
\%\end{array}$ & $\begin{array}{l}\leq 40 \mathrm{~m} / 35 \mathrm{f} \\
\%\end{array}$ & $\begin{array}{c}>40 \mathrm{~m} / 35 \mathrm{f} \\
\% \\
\end{array}$ \\
\hline \multicolumn{7}{|l|}{ Gender } \\
\hline Male & 91 & 44.0 & 36.2 & 19.8 & 87.9 & 12.1 \\
\hline Female & 98 & 61.2 & 26.5 & 12.2 & 83.7 & 16.3 \\
\hline p-value & & & $0.017 *$ & & \multicolumn{2}{|c|}{0.405} \\
\hline \multicolumn{7}{|l|}{ Ethnicity } \\
\hline FBCA & 66 & 62.1 & 30.3 & $7.6^{\mathrm{a}}$ & 89.4 & 10.6 \\
\hline USBCA & 62 & 41.9 & 40.3 & $17.7^{\mathrm{b}}$ & 83.9 & 16.1 \\
\hline $\mathrm{AA}$ & 61 & 54.0 & 23.0 & $23.0^{\mathrm{b}}$ & 83.6 & 16.4 \\
\hline $\mathrm{p}$-value & & & $0.034^{*}$ & & \multicolumn{2}{|c|}{0.570} \\
\hline \multicolumn{7}{|c|}{ Males by Ethnicity } \\
\hline FBCA & 31 & 58.0 & 35.5 & $6.5^{\mathrm{a}}$ & 93.5 & 6.5 \\
\hline USBCA & 30 & 26.7 & 53.3 & $20.0^{b}$ & 86.7 & 13.3 \\
\hline $\mathrm{AA}$ & 30 & 46.7 & 20.0 & $33.3^{\mathrm{b}}$ & 83.3 & 16.7 \\
\hline $\mathrm{p}$-value & & & $0.008 * *$ & & \multicolumn{2}{|c|}{0.458} \\
\hline \multicolumn{7}{|c|}{ Females by Ethnicity } \\
\hline FBCA & 35 & 65.7 & 25.7 & 8.6 & 88.6 & 11.4 \\
\hline USBCA & 32 & 56.3 & 28.1 & 15.6 & 81.3 & 18.8 \\
\hline $\mathrm{AA}$ & 31 & 61.3 & 25.8 & 12.9 & 83.9 & 16.1 \\
\hline p-value & & & 0.912 & & \multicolumn{2}{|c|}{0.885} \\
\hline
\end{tabular}

${ }^{*} \mathrm{p}<0.05 ; * \mathrm{p}<0.01$

${ }^{a, b}$ Values in a column with differing superscripts differed significantly at $\mathrm{p}<0.05$ (Bonferroni's test)

$\mathrm{BMI}=$ Body Mass Index; $\mathrm{WC}=$ Waist Circumference

FBCA $=$ Foreign-born Afro Caribbeans

USBCA = US-born Afro Caribbean Americans

$\mathrm{AA}=$ African Americans 


\section{Cigarette Smoking and Alcohol Consumption}

Although significantly $(p<0.05)$ more AA $(n=3,9.7 \%)$ females smoked cigarettes compared to FBCA $(0.0 \%)$ and USBCA $(0.0 \%)$ females, additional analysis using the Bonferroni's post hoc test showed no significant differences in smoking habits between the three females ethnic subgroups (Table 39). The percentage of FBCA $(n=16,24.2 \%)$ participants who consumed more than one alcoholic beverage per month was significantly $(\mathrm{p}<0.05)$ lower than AA $(n=31,50.8 \%)$ participants but not the USBCA $(n=18,29.0 \%)$ participants. However, further analysis using the Bonferroni's post hoc test showed no significant differences in alcohol consumption between the three ethnic groups (Table 39).

Similarly, the percentage of FBCA $(n=5,16.1 \%)$ males who consumed more than one alcoholic beverage per month was significantly $(\mathrm{p}<0.01)$ lower compared to USBCA $(\mathrm{n}=13,43.3 \%)$ and $\mathrm{AA}(\mathrm{n}=19,63.3 \%)$ participants, but further analysis using the Bonferroni's post hoc test showed no significant differences in alcohol consumption between the three male ethnic subgroups (Table 39). 
Table 39 - Distribution Cigarette Smoking and Alcohol Consumption of the of the Participants by Gender, Ethnicity and Gender-ethnicity Subgroups

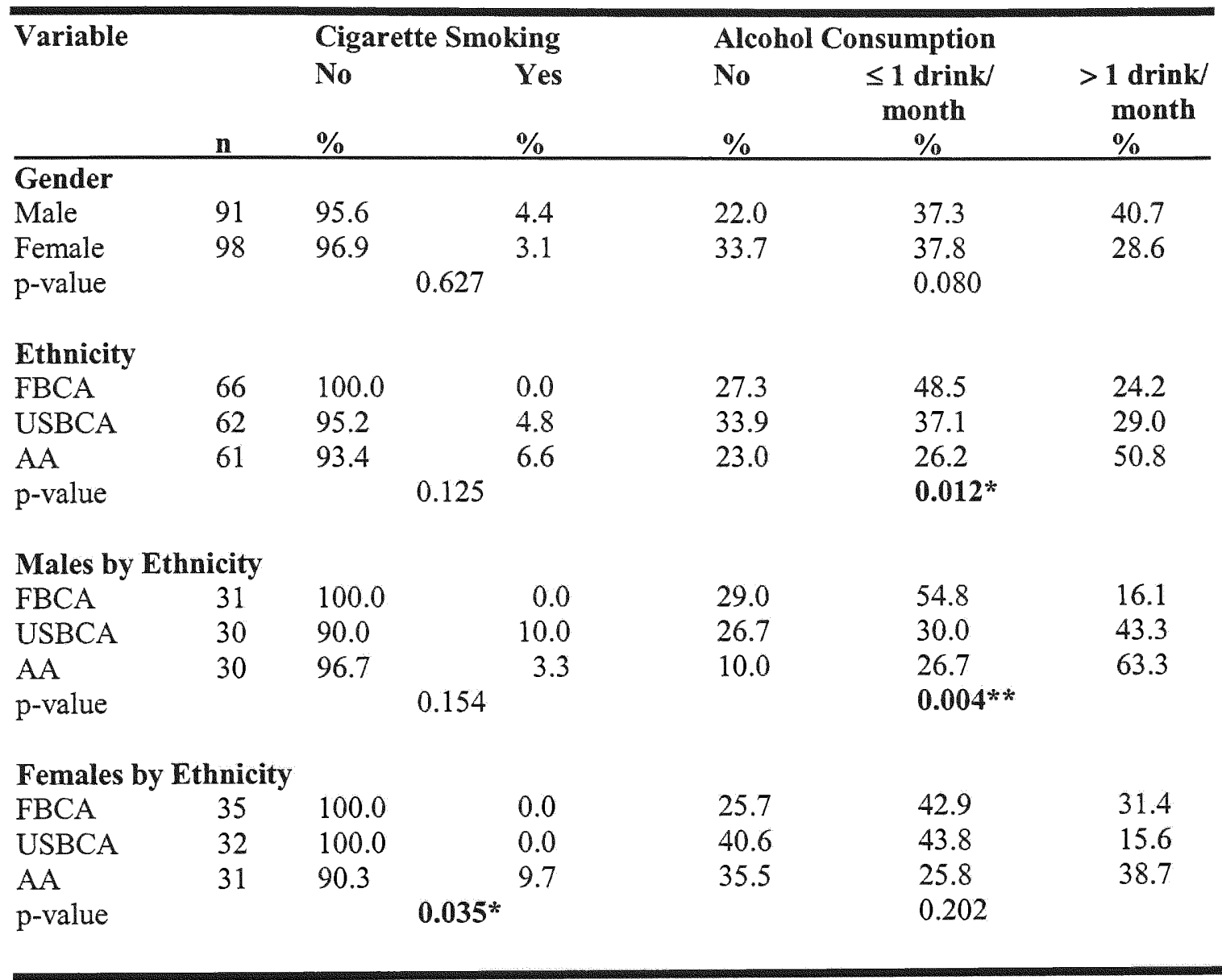

* $\mathrm{p}<0.05 ; * * \mathrm{p}<0.01$

FBCA $=$ Foreign-born Afro Caribbeans

USBCA = US-born Afro Caribbean Americans

$\mathrm{AA}=$ African Americans 
Section 3-Research Question 3: Are FBCA adults less likely to have physiological risk factors for CHD as compared to USBCA and AA adults?

Systolic Blood pressure (SBP) (> $120 \mathrm{~mm} \mathrm{Hg}$ ), diastolic blood pressure (DBP) (> $80 \mathrm{~mm} \mathrm{Hg}$ ), fasting blood glucose (FBG) (>110 mg/dL), total cholesterol (TC) $(\geq 200$ $\mathrm{mg} / \mathrm{dL})$, low-density lipoprotein cholesterol (LDL-C) $(\geq 130 \mathrm{mg} / \mathrm{dL})$ and triglyceride (TG) levels ( $\geq 200 \mathrm{mg} / \mathrm{dL}$ ) are considered elevated, whereas high-density lipoprotein cholesterol (HDL-C) levels $<40 \mathrm{mg} / \mathrm{dL}$, males and $<50 \mathrm{mg} / \mathrm{dL}$, females are considered undesirable. These risk factors are those currently used to evaluate risk for CHD (NCEP: ATP III, 2001; AHA Statistical Update, 2004).

\section{Blood Pressures}

The mean SBP $(\mathrm{mm} \mathrm{Hg})(111.9 \pm 11.7$ vs. $104.6 \pm 9.9)$ and DBP $(74.6 \pm 8.6$ vs. $69.9 \pm 8.8)$ of the males were significantly $(\mathrm{p}<0.001)$ higher than the females (Table 40$)$. Also, significantly $(\mathrm{p}<0.05)$ more males than females $(22.0 \%$ vs. $13.3 \%)$ had elevated SBP and DBP (35.2\% vs. $19.4 \%)$ (Table 41$)$.

There were no significant differences between the three ethnic groups or the three male and three female ethnic subgroups with respect to the mean SBP, DBP or the percentages of the three ethnic groups or the three male and three female ethnic subgroups with elevated SBP and DBP (Tables 40 and 41). 
Table 40 - Mean Blood Pressure Levels of Participants by Gender, Ethnicity and Gender-ethnicity Subgroups

\begin{tabular}{|c|c|c|c|}
\hline \multirow[t]{2}{*}{ Variable } & n & $\mathrm{SBP}(\mathrm{mm} \mathrm{Hg})$ & $\mathrm{DBP}(\mathrm{mm} \mathrm{Hg})$ \\
\hline & \multicolumn{3}{|c|}{ Mean $\pm S D$} \\
\hline \multicolumn{4}{|l|}{ Gender } \\
\hline Male & 91 & $111.9 \pm 11.7$ & $74.6 \pm 8.6$ \\
\hline Female & 98 & $104.6 \pm 9.9$ & $69.9 \pm 8.8$ \\
\hline p-value & & $0.001 * * *$ & $0.001 * * *$ \\
\hline \multicolumn{4}{|l|}{ Ethnicity } \\
\hline FBCA & 66 & $107.8 \pm 10.3$ & $72.6 \pm 9.1$ \\
\hline USBCA & 62 & $107.6 \pm 11.0$ & $71.5 \pm 8.9$ \\
\hline $\mathrm{AA}$ & 61 & $109.0 \pm 12.8$ & $72.4 \pm 9.3$ \\
\hline $\mathrm{p}$-value & & 0.753 & 0.790 \\
\hline \multicolumn{4}{|c|}{ Males by Ethnicity } \\
\hline FBCA & 31 & $110.3 \pm 12.0$ & $74.0 \pm 9.6$ \\
\hline USBCA & 30 & $111.0 \pm 10.2$ & $74.0 \pm 7.4$ \\
\hline $\mathrm{AA}$ & 30 & $114.3 \pm 12.8$ & $75.8 \pm 8.9$ \\
\hline $\mathrm{p}$-value & & 0.365 & 0.646 \\
\hline \multicolumn{4}{|c|}{ Females by Ethnicity } \\
\hline FBCA & 35 & $105.6 \pm 8.0$ & $71.3 \pm 8.5$ \\
\hline USBCA & 32 & $104.4 \pm 10.9$ & $69.2 \pm 9.6$ \\
\hline $\mathrm{AA}$ & 31 & $103.9 \pm 10.8$ & $69.0 \pm 8.4$ \\
\hline $\mathrm{p}$-value & & 0.773 & 0.513 \\
\hline
\end{tabular}

*** $\mathrm{p}<0.001$

$\mathrm{SBP}=$ Systolic Blood Pressure; DBP = Diastolic Blood Pressure

FBCA $=$ Foreign-born Afro Caribbeans

USBCA = US-born Afro Caribbean Americans

$\mathrm{AA}=$ African Americans 
Table 41 - Distribution of Blood Pressures of the Participants by Gender, Ethnicity and Gender-ethnicity Subgroups

\begin{tabular}{|c|c|c|c|c|c|}
\hline \multirow[t]{2}{*}{ Variable } & \multirow[t]{2}{*}{ n } & \multicolumn{2}{|c|}{$\mathrm{SBP}(\mathrm{mm} \mathrm{Hg})$} & \multicolumn{2}{|c|}{$\mathrm{DBP}(\mathrm{mm} \mathrm{Hg})$} \\
\hline & & $\begin{array}{l}<120 \\
\%\end{array}$ & $\begin{array}{l}\geq 20 \\
\%\end{array}$ & $\begin{array}{l}<80 \\
\%\end{array}$ & $\begin{array}{l}\geq 80 \\
\%\end{array}$ \\
\hline \multicolumn{6}{|l|}{ Gender } \\
\hline Male & 91 & 78.0 & 22.0 & 64.8 & 35.2 \\
\hline Female & 98 & 90.8 & 13.3 & 80.6 & 19.4 \\
\hline $\mathrm{p}$-value & \multicolumn{3}{|c|}{$0.015^{*}$} & \multicolumn{2}{|c|}{$0.015^{*}$} \\
\hline \multicolumn{6}{|l|}{ Ethnicity } \\
\hline FBCA & 66 & 84.8 & 15.2 & 72.2 & 27.3 \\
\hline USBCA & 62 & 87.1 & 12.9 & 79.0 & 21.0 \\
\hline $\mathrm{AA}$ & 61 & 80.0 & 18.0 & 67.2 & 32.8 \\
\hline $\mathrm{p}$-value & \multicolumn{3}{|c|}{0.731} & \multicolumn{2}{|c|}{0.335} \\
\hline \multicolumn{6}{|c|}{ Males by Ethnicity } \\
\hline FBCA & $31^{\circ}$ & 74.2 & 25.8 & 71.0 & 29.0 \\
\hline USBCA & 30 & 86.7 & 13.3 & 73.3 & 26.7 \\
\hline $\mathrm{AA}$ & 30 & 73.3 & 26.7 & 50.0 & 50.0 \\
\hline $\mathrm{p}$-value & \multicolumn{3}{|c|}{0.376} & \multicolumn{2}{|c|}{0.113} \\
\hline \multicolumn{6}{|c|}{ Females by Ethnicity } \\
\hline FBCA & 35 & 94.3 & 5.7 & 74.3 & 25.7 \\
\hline USBCA & 32 & 87.5 & 12.5 & 84.4 & 15.6 \\
\hline $\mathrm{AA}$ & 31 & 90.3 & 9.7 & 83.9 & 16.1 \\
\hline $\mathrm{p}$-value & \multicolumn{3}{|c|}{0.626} & \multicolumn{2}{|c|}{0.386} \\
\hline
\end{tabular}

$* \mathrm{p}<0.05$

$\mathrm{SBP}=$ Systolic Blood Pressure; DBP = Diastolic Blood Pressure

$\mathrm{FBCA}=$ Foreign-born Afro Caribbeans

USBCA = US-born Afro Caribbean Americans

$\mathrm{AA}=$ African Americans 


\section{Blood Glucose and Blood Lipids}

The mean FBG, TC, LDL-C, TG and VLDL values were not significantly different between the genders, the three ethnic groups or between the three male and three female ethnic subgroups (Table 42). Although no significant differences were observed between the percentages of participants with elevated FBG ( $\geq 110 \mathrm{mg} / \mathrm{dL})$, TC $(\geq 200 \mathrm{mg} / \mathrm{dL}), \mathrm{LDL}-\mathrm{C}(\geq 130 \mathrm{mg} / \mathrm{dL})$, and TG ( $\geq 150 \mathrm{mg} / \mathrm{dL})$, the percentages of those with elevated FBG levels ranged from $5.7 \%$ in FBCA females to $6.7 \%$ in USBCA and AA males, those with elevated TC ranged from $19.4 \%$ in FBCA males to $37.5 \%$ in USBCA females, those with elevated LDL-C ranged from $22.6 \%$ in FBCA males to $43.3 \%$ in USBCA males and those with elevated TG ranged from $8.6 \%$ in FBCA females to $16.1 \%$ in FBCA males (Table 43 ).

However, the mean HDL-C level $(\mathrm{mg} / \mathrm{dL})$ of the males $(43.7 \pm 7.7)$ was significantly $(\mathrm{p}<0.001)$ lower compared to the females $(55.0 \pm 12.5)$ (Table 42). Also, the percentage of the males $(35.2 \%)$ with undesirable HDL-C $(<40 \mathrm{mg} / \mathrm{dL})$ was significantly $(p<0.001)$ higher compared to the females $(8.2 \%)$ (Table 43$)$.

The mean HDL-C levels or the percentages of individuals with undesirable HDLC levels did not differ between the three ethnic groups (Tables 42 and 44). However, the percentage of the FBCA males (48.1\%) with undesirable HDL-C $(<40 \mathrm{mg} / \mathrm{dL})$ was higher than the USBCA (20.0\%) and the AA males (36.7\%) (Table 45) whereas, the percentage of USBCA (50.0\%) females with undesirable HDL-C level was higher compared to FBCA (31.4\%) and AA (19.4\%) females (Table 46). 


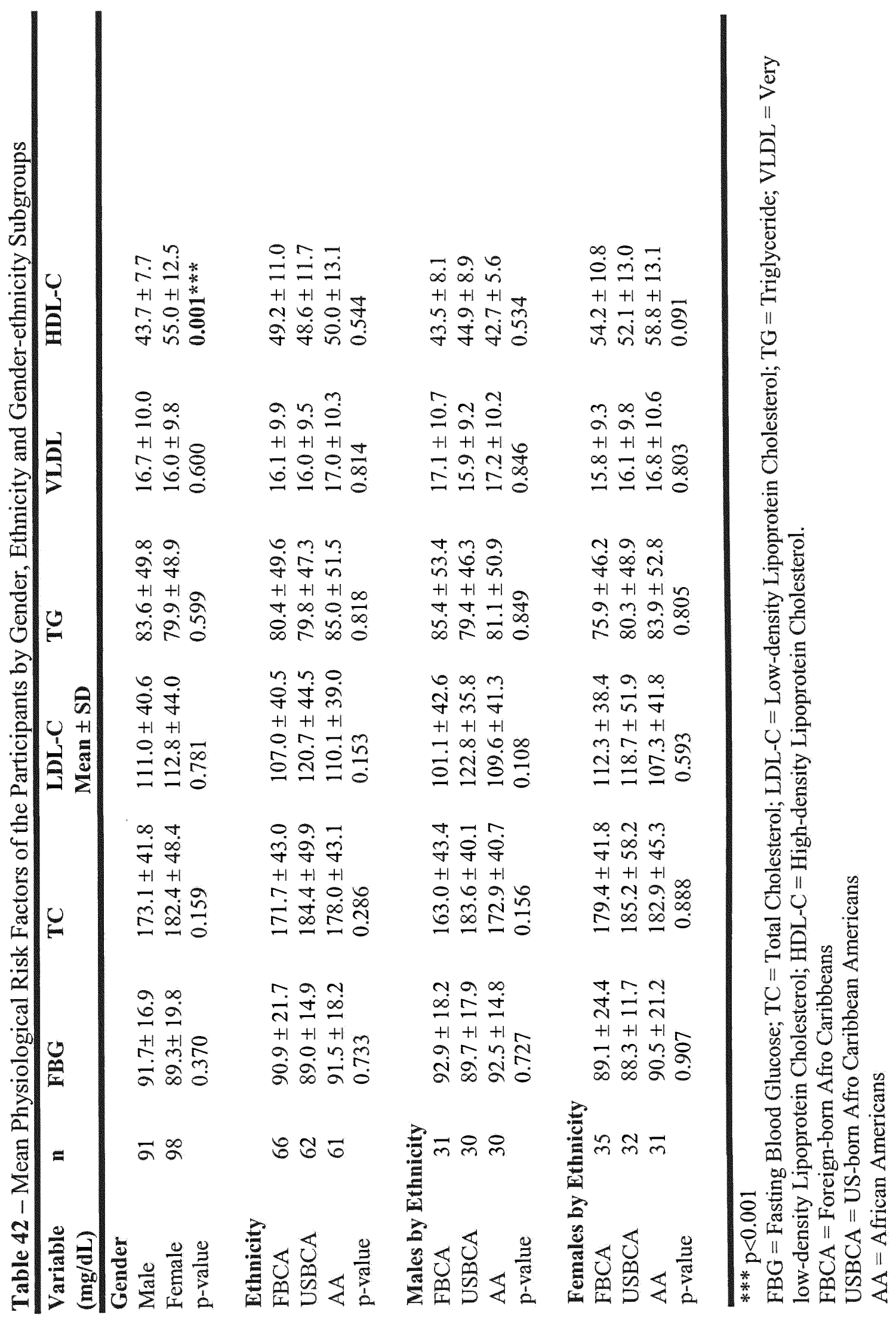


Table 43 - Distribution of Physiological Risk Factors of the Participants by Gender

\begin{tabular}{llll}
\hline Variables & Males & Females & $\mathbf{p}$ \\
& $\mathbf{n}=91$ \\
$\%$ & & $\begin{array}{l}\mathbf{n}=98 \\
\%\end{array}$ & \\
\hline
\end{tabular}

FBG (mg/dL)

$\begin{array}{lrrr}<110 & 93.4 & 93.9 & 0.894 \\ \geq 110 & 6.6 & 6.1 & \end{array}$

TC (mg/dL)

$\begin{array}{llll}<200 & 72.5 & 65.3 & 0.284 \\ \geq 200 & 27.5 & 34.7 & \end{array}$

LDL-C (mg/dL)

$\begin{array}{llll}<130 & 69.2 & 72.4 & 0.626 \\ \geq 130 & 30.8 & 27.6 & \end{array}$

TG $(\mathrm{mg} / \mathrm{dL})$

$\begin{array}{llll}<150 & 87.9 & 88.8 & 0.853 \\ \geq 150 & 12.1 & 11.2 & \end{array}$

HDL-C (mg/dL)

$\begin{array}{llll}<40^{\mathrm{m}} /<50^{\mathrm{f}} & 35.2 & 8.2 & \mathbf{0 . 0 0 1 * * *} \\ 40-59.9^{\mathrm{m}} / 50-59.9^{\mathrm{f}} & 61.6 & 61.2 & \\ \geq 60 & 3.3 & 30.6 & \end{array}$

\section{**: $p<0.001$}

$\mathrm{m}=$ male

$\mathrm{f}=$ female

FBG $=$ Fasting Blood Glucose $\geq 110 \mathrm{mg} / \mathrm{dL}$ undesirable; $\mathrm{TC}=$ Total Cholesterol $\geq 200 \mathrm{mg} / \mathrm{dL}=$ elevated; LDL-C $=$ Low-density Lipoprotein Cholesterol $\geq 130 \mathrm{mg} / \mathrm{dL}=$ Undesirable; HDL-C = High-density Lipoprotein Cholesterol $(\mathrm{mg} / \mathrm{dL})<40$ (males),$<50$ (females) = Undesirable; TG $=$ Triglyceride $\geq 150=$ undesirable (NCEP: ATP III, 2001; JNC VII, 2003). 
Table 44 - Distribution of Physiological Risk Factors of the Participants by Ethnicity

\begin{tabular}{|c|c|c|c|c|}
\hline Variables & $\begin{array}{l}\text { FBCA } \\
\mathrm{n}=66 \\
\% \\
\end{array}$ & $\begin{array}{l}\text { USBCA } \\
\mathrm{n}=62 \\
\% \\
\end{array}$ & $\begin{array}{l}\text { AA } \\
\mathrm{n}=61 \\
\%\end{array}$ & $\mathbf{p}$ \\
\hline \multicolumn{5}{|l|}{ FBG (mg/dL) } \\
\hline$<110$ & 93.9 & 93.5 & 93.4 & \multirow[t]{2}{*}{0.993} \\
\hline$\geq 110$ & 6.1 & 6.5 & 6.6 & \\
\hline \multicolumn{5}{|l|}{ TC (mg/dL) } \\
\hline$<200$ & 74.2 & 62.9 & 68.9 & \multirow[t]{2}{*}{0.384} \\
\hline$\geq 200$ & 25.8 & 37.1 & 31.1 & \\
\hline \multicolumn{5}{|l|}{ LDL-C (mg/dL) } \\
\hline$<130$ & 72.7 & 64.5 & 75.4 & \multirow[t]{2}{*}{0.380} \\
\hline$\geq 130$ & 27.3 & 35.5 & 24.6 & \\
\hline \multicolumn{5}{|l|}{$\mathbf{T G}(\mathrm{mg} / \mathrm{dL})$} \\
\hline$<150$ & 87.9 & 88.7 & 88.5 & \multirow[t]{2}{*}{0.988} \\
\hline$\geq 150$ & 12.1 & 11.3 & 11.5 & \\
\hline \multicolumn{5}{|l|}{ HDL-C (mg/dL) } \\
\hline$<40^{\mathrm{m}} / 50^{\mathrm{f}}$ & 39.4 & 35.5 & 29.5 & \multirow{3}{*}{0.425} \\
\hline $40-59.9^{m} / 50-59^{f}$ & 50.0 & 46.8 & 47.5 & \\
\hline$\geq 60$ & 10.6 & 17.7 & 23.0 & \\
\hline \multicolumn{5}{|l|}{$\mathrm{m}=\mathrm{male}$} \\
\hline \multicolumn{5}{|l|}{$\mathrm{f}=$ female } \\
\hline \multicolumn{5}{|c|}{$\begin{array}{l}\text { FBG }=\text { Fasting Blood Glucose } \geq 110 \mathrm{mg} / \mathrm{dL} \text { undesirable; TC }=\text { Total Cholesterol } \geq 200 \mathrm{mg} / \mathrm{dL}= \\
\text { elevated; LDL-C }=\text { Low-density Lipoprotein Cholesterol } \geq 130 \mathrm{mg} / \mathrm{dL}=\text { Undesirable; HDL-C }= \\
\text { High-density Lipoprotein Cholesterol }(\mathrm{mg} / \mathrm{dL})<40 \text { (males), }<50(\mathrm{females})=\text { Undesirable; TG } \\
=\text { Triglyceride } \geq 150=\text { undesirable (NCEP: ATP III, 2001; JNC VII, 2003). } \\
\text { FBCA = Foreign-born Afro Caribbeans } \\
\text { USBCA = US-born Afro Caribbean Americans } \\
\text { AA = African Americans }\end{array}$} \\
\hline
\end{tabular}


Table 45 - Distribution of the Physiological Risk Factors of the Male Participants by Ethnicity

\begin{tabular}{|c|c|c|c|c|}
\hline Variables & $\begin{array}{l}\text { FBCA } \\
\mathrm{n}=31 \\
\%\end{array}$ & $\begin{array}{l}\text { USBCA } \\
\mathrm{n}=30 \\
\%\end{array}$ & $\begin{array}{l}\mathbf{A A} \\
\mathrm{n}=\mathbf{3 0} \\
\%\end{array}$ & p \\
\hline \multicolumn{5}{|l|}{$\overline{\mathrm{FBG}(\mathrm{mg} / \mathrm{dL})}$} \\
\hline$<110$ & 93.5 & 93.3 & 93.3 & 0.999 \\
\hline$\geq 110$ & 6.5 & 6.7 & 6.7 & \\
\hline
\end{tabular}

TC $(\mathrm{mg} / \mathrm{dL})$

$\begin{array}{lllll}<200 & 80.6 & 63.3 & 73.3 & 0.315 \\ \geq 200 & 19.4 & 36.7 & 26.7 & \end{array}$

LDL-C (mg/dL)

$\begin{array}{llll}<130 & 77.4 & 56.7 & 73.3 \\ \geq 130 & 22.6 & 43.3 & 26.7\end{array}$

TG $(\mathrm{mg} / \mathrm{dL})$

$<150$

83.9

90.0

90.0

0.697

$\geq 150$

16.1

10.0

10.0

HDL-C (mg/dL)

$$
\begin{aligned}
& <40 \\
& 40-59.9 \\
& \geq 60
\end{aligned}
$$

$48.4^{\mathrm{a}}$
51.6
0.0

$20.0^{\mathrm{b}}$

73.3

$36.7^{\mathrm{ab}}$

0.002 **

6.7

60.0

3.3

**p $<0.01$

${ }^{a, b}$ Values in a row with differing superscripts differed significantly at $p<0.05$ (Bonferroni's test) FBG $=$ Fasting Blood Glucose $\geq 110 \mathrm{mg} / \mathrm{dL}$ undesirable; TC $=$ Total Cholesterol $\geq 200 \mathrm{mg} / \mathrm{dL}=$ elevated; LDL-C = Low-density Lipoprotein Cholesterol $\geq 130 \mathrm{mg} / \mathrm{dL}=$ Undesirable; HDL-C = High-density Lipoprotein Cholesterol $<40 \mathrm{mg} / \mathrm{dL}=$ Undesirable; $\mathrm{TG}=$ Triglyceride $\geq 150=$ undesirable (NCEP: ATP III, 2001; JNC VII, 2003).

FBCA $=$ Foreign-born Afro Caribbeans

USBCA = US-born Afro Caribbean Americans

$\mathrm{AA}=$ African Americans 
Table 46 - Distribution of the Physiological Risk Factors of the Female Participants by Ethnicity

\begin{tabular}{|c|c|c|c|c|}
\hline Variable & $\begin{array}{l}\text { FBCA } \\
\mathrm{n}=35 \\
\% \\
\end{array}$ & $\begin{array}{l}\text { USBCA } \\
\mathrm{n}=32 \\
\%\end{array}$ & $\begin{array}{l}\text { AA } \\
\mathrm{n}=\mathbf{3 1} \\
\%\end{array}$ & p \\
\hline \multicolumn{5}{|l|}{$\overline{\mathbf{F B G}(\mathrm{mg} / \mathrm{dL})}$} \\
\hline$<110$ & 94.3 & 93.8 & 93.5 & 0.384 \\
\hline$\geq 110$ & 5.7 & 6.2 & 6.6 & \\
\hline \multicolumn{5}{|l|}{$\mathrm{TC}(\mathrm{mg} / \mathrm{dL})$} \\
\hline$<200$ & 68.6 & 62.5 & 64.5 & 0.681 \\
\hline$\geq 200$ & 31.4 & 37.5 & 35.5 & \\
\hline \multicolumn{5}{|l|}{ LDL-C (mg/dL) } \\
\hline$<130$ & 68.5 & 71.9 & 77.4 & 0.525 \\
\hline$\geq 130$ & 31.4 & 30.0 & 23.3 & \\
\hline \multicolumn{5}{|l|}{ TG $(\mathrm{mg} / \mathrm{dL})$} \\
\hline$<150$ & 91.4 & 87.5 & 87.1 & 0.425 \\
\hline$\geq 150$ & 8.6 & 12.5 & 12.9 & \\
\hline \multicolumn{5}{|l|}{ HDL-C (mg/dL) } \\
\hline$<50$ & $31.4^{\mathrm{ab}}$ & $50.0^{\mathrm{b}}$ & $19.5^{\mathrm{a}}$ & $0.033^{*}$ \\
\hline $50.0-59.9$ & 48.6 & 21.9 & 38.7 & \\
\hline$\geq 60$ & 20.0 & 28.1 & 41.9 & \\
\hline
\end{tabular}

$* \mathrm{p}<0.05$

${ }^{a, b}$ Values in a row with differing superscripts differed significantly at $\mathrm{p}<0.05$ (Bonferroni's test) FBG $=$ Fasting Blood Glucose $\geq 110 \mathrm{mg} / \mathrm{dL}$ undesirable; TC $=$ Total Cholesterol $\geq 200 \mathrm{mg} / \mathrm{dL}=$ elevated; LDL-C = Low-density Lipoprotein Cholesterol $\geq 130 \mathrm{mg} / \mathrm{dL}=$ Undesirable; HDL-C $=$ High-density Lipoprotein Cholesterol $<50 \mathrm{mg} / \mathrm{dL}=$ Undesirable; $\mathrm{TG}=$ Triglyceride $\geq 150=$ undesirable (NCEP: ATP III, 2001; JNC 7, 2003).

FBCA $=$ Foreign-born Afro Caribbeans

USBCA = US-born Afro Caribbean Americans

$\mathrm{AA}=$ African Americans 


\section{Section 4 - Research Question 4: Are FBCA Less Likely to have Emerging Risk Factors for Coronary Heart Disease compared to USBCA and AA Adults?}

\section{High-sensitivity C-Reactive Protein}

It is suggested that high-sensitivity CRP (hs-CRP) levels greater than $10 \mathrm{mg} / \mathrm{L}$ should not be used to assess CVD risk since this may reflect ongoing infection, systemic inflammation or trauma (Pearson et al., 2003). Thus, individuals ( 3 males; 12 females) with hs-CRP levels greater than $10 \mathrm{mg} / \mathrm{L}$ were excluded from data analysis in this study (. Participants with hs-CRP levels greater than $3.0 \mathrm{mg} / \mathrm{L}$ were considered to be at increased risk for CHD (Pearson et al., 2003).

Comparing by ethnicity, the mean hs-CRP levels of the FBCA $(1.1 \pm 1.3 \mathrm{mg} / \mathrm{L})$ and USBCA participants $(1.3 \pm 1.6 \mathrm{mg} / \mathrm{L})$ were significantly $(\mathrm{p}<0.01)$ lower compared to the AA participants $(2.2 \pm 2.7 \mathrm{mg} / \mathrm{L})$ (Table 47$)$. Comparing by female ethnic subgroups, the mean hs-CRP levels $(\mathrm{mg} / \mathrm{L})$ of the FBCA $(1.0 \pm 1.3)$ and USBCA females $(1.1 \pm 1.4)$ were significantly $(\mathrm{p}<0.05)$ lower compared to the AA females $(2.4 \pm 2.8)$ (Table 47$)$.

\section{Total Cholesterol/High-density Lipoprotein Cholesterol (TC/HDL-C) Ratio}

Participants with a TC/HDL-C ratio $\geq 5.0$ were considered to be at increased risk for CHD (Rifai and Ridker, 2001; Natarajan et al., 2003). In our study, the mean TC/HDL-C ratio of the males $(4.0 \pm 1.2)$ was significantly $(\mathrm{p}<0.01)$ higher than females $(3.5 \pm 1.4)$ (Table 47). Also, the percentage of the males (18.7\%) with elevated TC/HDL-C ratio ( $\geq$ 5.0) was significantly $(p<0.001)$ higher than that of the females $(12.23 \%)$ (Table 48$)$. 
Table 47 - Mean Emerging Risk Factors of the Participants by Gender, ethnicity and Gender-ethnicity Subgroups

\begin{tabular}{|c|c|c|c|c|c|c|}
\hline Variables & $\mathbf{n}$ & $\begin{array}{c}\text { hs-CRP } \\
(\mathrm{mg} / \mathrm{L})\end{array}$ & n & $\begin{array}{l}\text { TC/HDL-C } \\
\text { Ratio } \\
\text { Mean } \pm \text { SD }\end{array}$ & $\mathbf{n}$ & $\begin{array}{l}\text { Homocysteine } \\
(\mu \mathrm{mol} / \mathrm{L})\end{array}$ \\
\hline \multicolumn{7}{|l|}{ Gender } \\
\hline Male & 88 & $1.6 \pm 2.0$ & 91 & $4.0 \pm 1.2$ & 88 & $7.4 \pm 2.2$ \\
\hline Female & 86 & $1.5 \pm 2.1$ & 98 & $3.5 \pm 1.4$ & 95 & $6.0 \pm 2.3$ \\
\hline $\mathrm{p}$-value & & 0.803 & & $0.003^{* *}$ & & $0.001 * * *$ \\
\hline \multicolumn{7}{|l|}{ Ethnicity } \\
\hline FBCA & 57 & $1.1 \pm 1.3^{\mathrm{a}}$ & 66 & $3.6 \pm 1.3$ & 62 & $6.6 \pm 2.7$ \\
\hline USBCA & 57 & $1.3 \pm 1.6^{2}$ & 62 & $4.0 \pm 1.5$ & 60 & $6.6 \pm 1.8$ \\
\hline $\mathrm{AA}$ & 60 & $2.2 \pm 2.7^{\mathrm{b}}$ & 61 & $3.6 \pm 1.1$ & 61 & $6.8 \pm 2.5$ \\
\hline $\mathrm{p}$-value & & $0.004^{* *}$ & & 0.190 & & 0.895 \\
\hline \multicolumn{7}{|c|}{ Males by Ethnicity } \\
\hline FBCA & 28 & $1.2 \pm 1.2$ & 31 & $3.8 \pm 1.3$ & 28 & $7.4 \pm 1.5$ \\
\hline USBCA & 30 & $1.4 \pm 1.8$ & 30 & $4.2 \pm 1.1$ & 30 & $7.4 \pm 1.7$ \\
\hline $\mathrm{AA}$ & 30 & $2.1 \pm 2.7$ & 30 & $4.1 \pm 1.2$ & 30 & $7.4 \pm 3.2$ \\
\hline p-value & & 0.219 & & 0.458 & & 0.999 \\
\hline \multicolumn{7}{|c|}{ Females by Ethnicity } \\
\hline FBCA & 29 & $1.0 \pm 1.3^{\mathrm{a}}$ & 35 & $3.5 \pm 1.2$ & 34 & $6.0 \pm 3.2$ \\
\hline USBCA & 27 & $1.1 \pm 1.4^{\mathrm{a}}$ & 32 & $3.8 \pm 1.8$ & 30 & $5.8 \pm 1.5$ \\
\hline $\mathrm{AA}$ & 30 & $2.4 \pm 2.8^{b}$ & 31 & $3.2 \pm 0.8$ & 31 & $6.2 \pm 1.5$ \\
\hline $\mathrm{p}$-value & & $0.013^{*}$ & & 0.169 & & 0.799 \\
\hline
\end{tabular}

${ }^{*} \mathrm{p}<0.05 ;{ }^{* *} \mathrm{p}<0.01 ;{ }^{* * *} \mathrm{p}<0.001$

$a, b$ Values in a column with differing superscripts differed significantly at $p<0.05$ (Bonferroni test) hs-CRP = high-sensitivity C-reactive Protein

TC/HDL-C $=$ Total Cholesterol/High-density Lipoprotein Cholesterol Ratio

FBCA $=$ Foreign-born Afro Caribbeans

USBCA = US-born Afro Caribbean Americans

$\mathrm{AA}=$ African Americans 


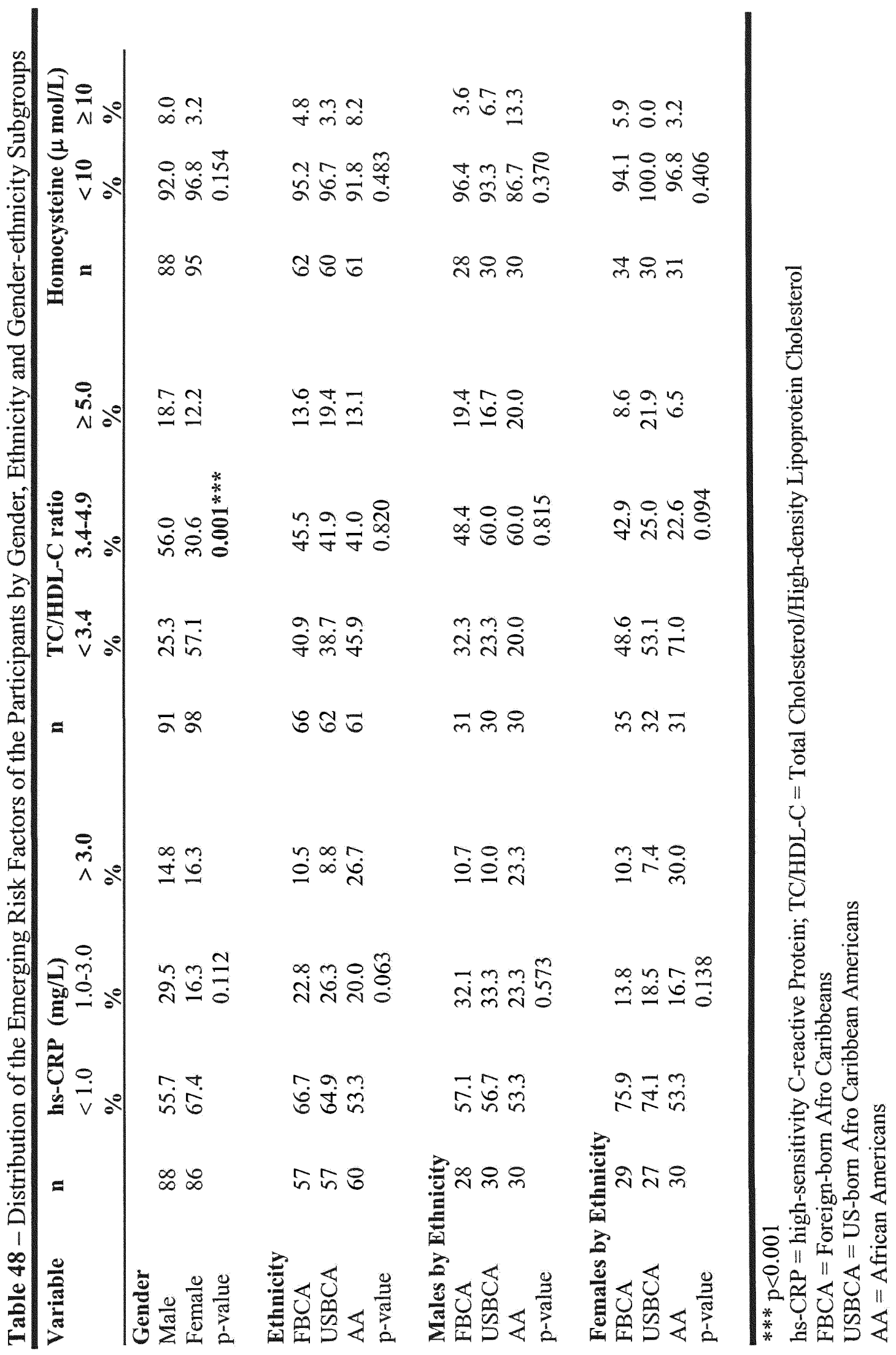




\section{Homocysteine}

Homocysteine levels greater than $10 \mu \mathrm{mol} / \mathrm{L}$ were considered elevated (Malinow et al., 1999). In the present study, the mean homocysteine level $(\mu \mathrm{mol} / \mathrm{L})$ of males $(7.4 \pm$ 2.2) was significantly $(\mathrm{p}<0.001)$ higher than that of the females $(6.0 \pm 2.3)$ (Table 47).

\section{Additional Findings Clustering of Behavioral and Metabolic Risk Factors}

The risk of CHD rises sharply as the number of risk factors increase. Individuals in whom $\mathrm{CHD}$ develops generally have more than one risk factor.

For the purposes of this study behavioral and metabolic risk factors were organized into 2 categories:

1. Behaviora1/Environmental Risks (diet score $0-2$, watched TV often/very often (an indication of sedentary lifestyle), BMI $\geq 25.0$, cigarette smoking, consuming alcoholic beverages $>$ once per month) were each given a score of 1 and, (diet score $\geq 3$, watched TV never/sometimes, BMI $<25.0$, no cigarette smoking, consuming alcoholic beverages < once per month) were each given a score of 0 . The scores were summed across the selected behavioral/environmental risks to give a total score. Higher scores were indicative of 'poor' behavioral/environmental habits.

2. According to the NCEP: ATP III Panel, individuals with 3 of the following 5 factors: (1) abdominal obesity - waist circumference $>40$ inches in men and $>$ 35 inches in women; (2) S/DBP $\geq 130 / 85 \mathrm{~mm} \mathrm{Hg}$; (3) FBG $\geq 110 \mathrm{mg} / \mathrm{dL}$; (4) $\mathrm{TG} \geq 150 \mathrm{mg} / \mathrm{dL} ;(5) \mathrm{HDL}-\mathrm{C}<40 \mathrm{mg} / \mathrm{dL}$, were classified as meeting the 
criteria for the metabolic syndrome (MS). Waist circumference was used because it correlates more strongly with abdominal obesity than BMI. An additional evaluation of the individuals classified with or without the MS was done using the new guideline for BP cut-points (JNC VII, 2004).

The mean behavioral/environmental risk factors for CHD was significantly $(\mathrm{p}<$ $0.001)$ lower in females $(1.2 \pm 0.9)$ compared to males $(1.9 \pm 1.1)$. Significantly $(p<0.01)$ more males $(24.2 \%)$ than females $(8.2 \%)$ had three or more behavioral risk factors (Table 49).

By ethnicity, the mean behavioral/environmental risk factors for $\mathrm{CHD}$ was significantly $(\mathrm{p}<0.01)$ lower in FBCA $(1.2 \pm 0.8)$ participants compared to USBCA $(1.7$ $\pm 1.2)$ and AA $(1.7 \pm 1.0)$ participants (Table 49). Significantly $(\mathrm{p}<0.05)$ more USBCA $(22.6 \%)$ and $\mathrm{AA}(19.7 \%)$ participants had 3 or more behavioral/environmental risk factors compared to the FBCA (6.1\%) participants (Table 49). By ethnicity subgroups, the mean behavioral/environment risk factors for CHD of the FBCA $(1.2 \pm 0.8)$ males was significantly $(\mathrm{p}<0.001)$ lower compared to the USBCA $(2.4 \pm 1.2)$ and AA $(2.0 \pm$ 1.0) males (Table 49). Similarly, the percentages of USBCA (36.7\%) and AA (33.3\%) males with 3 or more behavioral/environmental risk factors were significantly $(p<0.01)$ higher compared to FBCA (3.2\%) males (Table 49). 
Table 49 - Mean and Frequency Distribution of the Behavioral/Environmental Risk

Factors of Participants by Gender, Ethnicity and Gender-ethnicity Subgroups

\begin{tabular}{|c|c|c|c|c|c|}
\hline \multirow[t]{4}{*}{ Variables } & \multirow[t]{3}{*}{ n } & \multicolumn{4}{|c|}{ Behavioral/Environmental Risk Factors } \\
\hline & & & Risk & Categories $^{1}$ & \\
\hline & & & 0 & $1-2$ & $\geq 3$ \\
\hline & n & Mean \pm SD & $\%$ & $\%$ & $\%$ \\
\hline \multicolumn{6}{|l|}{ Gender } \\
\hline Male & 91 & $1.9 \pm 1.1$ & 9.9 & 65.9 & 24.2 \\
\hline Female & 98 & $1.2 \pm 0.9$ & 18.8 & 73.5 & 8.2 \\
\hline p-value & & $0.001 * * *$ & & $0.006^{* *}$ & \\
\hline \multicolumn{6}{|l|}{ Ethnicity } \\
\hline $\mathrm{FBCA}$ & 66 & $1.2 \pm 0.8^{\mathrm{a}}$ & 21.2 & 72.7 & $6.1^{\mathrm{a}}$ \\
\hline USBCA & 62 & $1.7 \pm 1.2^{\mathrm{b}}$ & 11.3 & 66.1 & $22.6^{b}$ \\
\hline AA & 61 & $1.7 \pm 1.0^{\mathrm{b}}$ & 9.8 & 70.5 & $19.7^{\mathrm{b}}$ \\
\hline p-value & & $0.01 * *$ & & $0.041^{*}$ & \\
\hline \multicolumn{6}{|c|}{ Males by Ethnicity } \\
\hline FBCA & 31 & $1.2 \pm 0.8^{\mathrm{a}}$ & 22.6 & 74.2 & $3.2^{\mathrm{a}}$ \\
\hline USBCA & 30 & $2.4 \pm 1.2^{b}$ & 0.0 & 63.3 & $36.7^{\mathrm{b}}$ \\
\hline $\mathrm{AA}$ & 30 & $2.0 \pm 1.0^{b}$ & 6.7 & 60.0 & $33.3^{\mathrm{b}}$ \\
\hline p-value & & $0.0001 * * *$ & & $0.002^{* *}$ & \\
\hline \multicolumn{6}{|c|}{ Females by Ethnicity } \\
\hline FBCA & 35 & $1.2 \pm 0.9$ & 20.0 & 71.4 & 8.6 \\
\hline USBCA & 32 & $1.1 \pm 0.9$ & 21.9 & 68.8 & 9.4 \\
\hline $\mathrm{AA}$ & 31 & $1.5 \pm 0.8$ & 12.9 & 80.6 & 6.5 \\
\hline p-value & & 0.258 & & 0.865 & \\
\hline
\end{tabular}

${ }^{*} \mathrm{p}<0.05 ; * * \mathrm{p}<0.01 ; * * * \mathrm{p}<0.001$

a,b Values in a column with differing superscripts differed significantly at $p<0.05$ (Bonferroni's test)

${ }^{1}$ Range $0-5$ for Behavioral/Environmental Risks

FBCA $=$ Foreign-born Afro Caribbeans

USBCA = US-born Afro Caribbean Americans

$\mathrm{AA}=$ African Americans 
The NCEP: ATP III (2001) criteria for the MS were detected in $2.1 \%$ of this population (males, 2.2\%; females, $2.0 \%$ ). However, significantly $(\mathrm{p}<0.05)$ more female $(71.4 \%)$ than male (49.5\%) participants had no risk factors for the MS (Table 50). There was no significant difference in the percentages of FBCA (1.5\%), USBCA (1.6\%) or AA (3.2\%) participants who met the criteria for the MS (Table 50). When the JNC VII Guidelines for elevated blood pressure (S/DBP $\geq 120 / 80 \mathrm{~mm} \mathrm{Hg}$ ) were used, with respect to the metabolic syndrome, an additional 4 males met the criteria for the MS compared to the females (6.6\% vs. $2.0 \%)$ (Table 51$)$. 
Table 50 - Risk Factor Categories for the Metabolic Syndrome of Participants by Gender, Ethnicity and Gender-ethnicity Subgroups

\begin{tabular}{|c|c|c|c|c|}
\hline \multirow[t]{2}{*}{ Variables } & \multirow[b]{2}{*}{$\mathbf{n}$} & \multicolumn{3}{|c|}{ Number of Risk Factors for the Metabolic Syndrome } \\
\hline & & $\begin{array}{l}0 \\
\% \\
\end{array}$ & $\begin{array}{l}1-2 \\
\%\end{array}$ & $\begin{array}{l}\geq 3 \text { (MS) } \\
\%\end{array}$ \\
\hline \multicolumn{5}{|l|}{ Gender } \\
\hline Male & 91 & 49.5 & 48.4 & 2.2 \\
\hline Female & 98 & 71.4 & 26.5 & 2.0 \\
\hline p-value & & & $0.023 *$ & \\
\hline \multicolumn{5}{|l|}{ Ethnicity } \\
\hline FBCA & 66 & 59.1 & 39.4 & 1.5 \\
\hline USBCA & 62 & 61.3 & 37.1 & 1.6 \\
\hline AA & 61 & 62.3 & 34.4 & 3.2 \\
\hline p-value & & & 0.932 & \\
\hline \multicolumn{5}{|c|}{ Males by Ethnicity } \\
\hline FBCA & 31 & 41.9 & 58.1 & 0.0 \\
\hline USBCA & 30 & 56.7 & 43.3 & 0.0 \\
\hline $\mathrm{AA}$ & 30 & 50.0 & 43.3 & 6.7 \\
\hline $\mathrm{p}$-value & & & 0.642 & \\
\hline \multicolumn{5}{|c|}{ Females by Ethnicity } \\
\hline FBCA & 35 & 74.3 & 22.9 & 2.9 \\
\hline USBCA & 32 & 65.6 & 31.3 & 3.1 \\
\hline $\mathrm{AA}$ & 31 & 74.2 & 25.8 & 0.0 \\
\hline p-value & & & 0.865 & \\
\hline \multicolumn{5}{|l|}{$* \mathrm{p}<0.05$} \\
\hline \multicolumn{5}{|c|}{$\mathrm{MS}=$ Metabolic Syndrome } \\
\hline \multicolumn{5}{|c|}{ FBCA = Foreign-born Afro Caribbeans } \\
\hline \multicolumn{5}{|c|}{ USBCA = US-born Afro Caribbean Americans } \\
\hline $\mathrm{AA}=$ & & & & \\
\hline
\end{tabular}


Table 51 - Risk Factor Categories for the Metabolic Syndrome of Participants by Gender, Ethnicity and Gender-ethnicity Subgroups Using JNC VII Cut-points for Blood Pressure

Variables

(2)

1

\section{Gender}

Male

Female

p-value

91

91
98

66.3

Number of Risk Factors for the Metabolic Syndrome

0

$\%$

45.1
66.3

$1-2$

$\%$

48.4

31.7

$0.039 *$

Ethnicity

AA

37.8

4.5

40.3

$\geq 3$ (MS)

$\%$

32.8

6.5

0.867

6.6

2.0

54.9

6.5

46.7

43.3

0.0

0.505

13.4
FBCA

USBCA

66

62

61

p-value

Males by Ethnicity

FBCA 31

USBCA 30

AA 30

p-value
38.7

53.5

43.3

68.6

62.5

67.7
54.5

58.1

55.7

Females by Ethnicity

FBCA $\quad 35$

USBCA $\quad 32$

AA 31

p-value

$31 \quad 67.7$

* $\mathrm{p}<0.05$

MS = Metabolic Syndrome

FBCA $=$ Foreign-born Afro Caribbeans

USBCA = US-born Afro Caribbean Americans

$\mathrm{AA}=$ African Americans 


\section{Section 5-Relationships between Socio-demographic Variables and Behavioral/Environmental Risk Factors in FBCA, USBCA and AA Participants}

There were no associations between either education or household income (proxy for socio-economic status) with the behavioral/environmental risk factors by gender (Table 52). Age correlated positively with the diet score $(\mathrm{r}=0.245 ; \mathrm{p}<0.05)$ and positively with body mass index (BMI) in males but not in females, whereas age correlated positively with cigarette smoking in the females. Family history of CHD was positively associated with cigarette smoking $(r=0.213 ; \mathrm{p}<0.05)$ in males but not females (Table 52).

Like the gender groups, there were no associations between the socio-economic status (SES) and the behavioral/environmental risk factors by ethnicity (Table 53). In the FBCA and USBCA participants, age was positively associated with BMI ( $r=0.277, \mathrm{p}<$ $0.05 ; \mathrm{r}=0.277, \mathrm{p}<0.05$, respectively). Also, age was positively associated with the diet score $(r=0.321 ; \mathrm{p}<0.05)$ in FBCA participants and positively with cigarette smoking $(\mathrm{r}=0.413 ; \mathrm{p}<0.01)$ in AA participants (Table 53). Knowledge of CHD risk factors was negatively associated with the physical activity index $(\mathrm{PAI})(\mathrm{r}=-0.413 ; \mathrm{p}<0.01)$ in the FBCA participants and positively associated with BMI in AA participants (Table 53). 
Table 52 - Correlations between Selected Socio-demographic Variables and the Behavioral/Environmental Risk Factors of the Participants by Gender

\begin{tabular}{lcc}
\hline Variables & $\begin{array}{c}\text { Males } \\
\mathbf{n}=91\end{array}$ & $\begin{array}{c}\text { Females } \\
\mathbf{n}=98\end{array}$ \\
\hline Age & & -0.159 \\
Diet Score & $\mathbf{0 . 2 4 5 ^ { * }}$ & 0.054 \\
Physical Activity Index & -0.096 & 0.080 \\
Body Mass Index & $\mathbf{0 . 2 9 6 ^ { * * }}$ & $\mathbf{0 . 2 5 9 ^ { * }}$ \\
Cigarette Smoking & 0.098 & 0.122 \\
Alcohol Intake & -0.073 & \\
Education & & 0.105 \\
Diet Score & -0.154 & -0.141 \\
Physical Activity Index & -0.064 & 0.010 \\
Body Mass Index & 0.102 & -0.073 \\
Cigarette Smoking & -0.145 & 0.132 \\
Alcohol Intake & -0.045 & \\
& & -0.023 \\
Household Income & & 0.037 \\
Diet Score & -0.121 & -0.111 \\
Physical Activity Index & -0.159 & -0.024 \\
Body Mass Index & 0.111 & 0.069 \\
Cigarette Smoking & -0.084 & \\
Alcohol Intake & -0.004 & -0.109 \\
& & -0.061 \\
Knowledge of CHD Risks & & 0.064 \\
Diet Score & 0.056 & 0.069 \\
Physical Activity Index & -0.158 & 0.020 \\
Body Mass Index & 0.175 & \\
Cigarette Smoking & 0.027 & 0.034 \\
Alcohol Intake & -0.031 & 0.064 \\
& & 0.010 \\
Family History of CHD & 0.058 & 0.020 \\
Diet Score & -0.071 & \\
Physical Activity Index & -0.019 & \\
Body Mass Index & $\mathbf{0 . 2 1 3 *}$ & \\
Cigarette Smoking & 0.064 & \\
Alcohol Intake & & \\
& & \\
\hline
\end{tabular}

* $p<0.05 ;{ }^{*} \mathrm{p}<0.01$

$\mathrm{CHD}=$ Coronary Heart Disease 
Table 53 - Correlations between Selected Socio-demographic Variables and the behavioral/Environmental Risk Factors of the Participants by Ethnicity

\begin{tabular}{|c|c|c|c|}
\hline Variables & $\begin{array}{r}\text { FBCA } \\
\text { n=66 } \\
\end{array}$ & $\begin{array}{l}\text { USBCA } \\
\mathrm{n}=62\end{array}$ & $\begin{array}{c}\mathbf{A A} \\
\mathrm{n}=61\end{array}$ \\
\hline \multicolumn{4}{|l|}{$\overline{\text { Age }}$} \\
\hline Diet Score & $0.321^{*}$ & -0.140 & 0.016 \\
\hline Physical Activity Index & 0.017 & -0.207 & 0.139 \\
\hline Body Mass Index & $0.277^{*}$ & $0.255^{*}$ & 0.204 \\
\hline Cigarette Smoking & -- & 0.043 & $0.413^{* *}$ \\
\hline Alcohol Intake & -0.032 & 0.083 & -0.002 \\
\hline \multicolumn{4}{|l|}{ Knowledge of CHD Risks } \\
\hline Diet Score & -0.045 & 0.003 & -0.182 \\
\hline Physical Activity Index & $-0.413 * *$ & -0.102 & 0.059 \\
\hline Body Mass Index & -0.039 & -0.130 & $0.378^{* *}$ \\
\hline Cigarette Smoking & -- & 0.020 & 0.031 \\
\hline Alcohol Intake & 0.081 & -0.003 & -0.109 \\
\hline \multicolumn{4}{|l|}{ Family History of CHD } \\
\hline Diet Score & 0.085 & 0.077 & -0.132 \\
\hline Physical Activity Index & -0.060 & -0.191 & 0.008 \\
\hline Body Mass Index & -0.039 & 0.184 & -0.004 \\
\hline Cigarette Smoking & -- & 0.152 & 0.154 \\
\hline Alcohol Intake & -0.011 & 0.102 & 0.093 \\
\hline \multicolumn{4}{|l|}{ Education } \\
\hline Diet Score & -0.044 & -0.153 & 0.087 \\
\hline Physical Activity Index & -0.162 & -0.192 & 0.001 \\
\hline Body Mass Index & 0.080 & 0.023 & 0.072 \\
\hline Cigarette Smoking & -- & -0.203 & -0.111 \\
\hline Alcohol Intake & 0.085 & 0.021 & 0.009 \\
\hline \multicolumn{4}{|l|}{ Household Income } \\
\hline Diet Score & 0.005 & -0.150 & -0.014 \\
\hline Physical Activity Index & -0.112 & 0.140 & -0.068 \\
\hline Body Mass Index & -0.054 & 0.012 & 0.089 \\
\hline Cigarette Smoking & - & -0.142 & 0.003 \\
\hline Alcohol Intake & 0.114 & 0.077 & -0.081 \\
\hline
\end{tabular}

\footnotetext{
${ }^{*} \mathrm{p}<0.05 ;{ }^{* *} \mathrm{p}<0.01$

$\mathrm{CHD}=$ Coronary Heart Disease

FBCA $=$ Foreign-born Afro Caribbeans

USBCA = US-born Afro Caribbean Americans

$\mathrm{AA}=$ African Americans
} 


\section{Relationships between Socio-demographic Variables and Physiological Risk Factors}

In females, age was positively associated with diastolic blood pressure (DBP) $(r=0.231 ; p<0.05)$. In males, education was negatively associated with systolic blood pressure $(\mathrm{SBP})(\mathrm{r}=-0.227 ; \mathrm{p}<0.05)$ whereas in females, education was positively associated with total cholesterol $(\mathrm{TC})(\mathrm{r}=0.254 ; \mathrm{p}<0.05)$ and low-density lipoprotein cholesterol (LDL-C) $(r=0.257 ; \mathrm{p}<0.05)$ (Table 54). In males, the perceived stress (PS) score was positively associated with HDL-C $(r=0.210 ; \mathrm{p}<0.05)$ (Table 54).

In the AA participants, age was positively associated with $\mathrm{SBP}(\mathrm{r}=0.500 ; \mathrm{p}<0.01)$ and DBP $(r=0.406 ; p<0.01)$ (Table 55). In USBCA participants, education was positively associated with total cholesterol (TC) $(r=0.307 ; \mathrm{p}<0.05)$ and, in USBCA and AA participants, positively associated with LDL-C $(r=0.302, p<0.05 ; \mathrm{r}=0.309 ; \mathrm{p}<0.05)$, respectively (Table 55). In the FBCA participants, household income was negatively associated with TC $(r=-0.297 ; \mathrm{p}<0.05)$ and LDL-C $(r=-0.329 ; \mathrm{p}<0.01)$ (Table 55). The PS score in USBCA participants was negatively associated with SBP $(r=-0.377$; $p<$ $0.01)$ and DBP $(r=-0.365 ; \mathrm{p}<0.01)$. Also, the PS score was positively associated with fasting blood glucose $(\mathrm{FBG})(\mathrm{r}=0.284 ; \mathrm{p}<0.05)$ in AA participants whereas, in FBCA participants, the PS score was positively associated with high-density lipoprotein cholesterol (HDL-C) (Table 55). 
Table 54 - Correlations between Selected Socio-demographic Variables and the Physiological Risk Factors of the Participants by Gender

\begin{tabular}{lcc}
\hline Variables & Males & Females \\
$\mathbf{n}=\mathbf{9 1}$ & $\mathbf{n}=\mathbf{9 8}$ \\
\hline Age & & \\
Systolic Blood Pressure & 0.129 & 0.163 \\
Diastolic Blood Pressure & 0.080 & $\mathbf{0 . 2 3 1 ^ { * }}$ \\
Fasting Blood Glucose & 0.037 & -0.076 \\
Total Cholesterol & 0.149 & 0.018 \\
LDL-C & 0.045 & 0.066 \\
TG & -0.034 & -0.106 \\
HDL-C & 0.151 & -0.089 \\
Education & & \\
Systolic Blood Pressure & $\mathbf{0 . 2 2 7 ^ { * }}$ & 0.167 \\
Diastolic Blood Pressure & -0.129 & 0.174 \\
Fasting Blood Glucose & 0.072 & 0.007 \\
Total Cholesterol & 0.030 & $\mathbf{0 . 2 5 4}$ \\
LDL-C & 0.036 & $\mathbf{0 . 2 5 7}$ \\
TG & 0.030 & -0.081 \\
HDL-C & -0.122 & 0.094 \\
Household Income & & \\
Systolic Blood Pressure & 0.019 & 0.003 \\
Diastolic Blood Pressure & -0.076 & 0.131 \\
Fasting Blood Glucose & 0.045 & -0.026 \\
Total Cholesterol & -0.128 & -0.118 \\
LDL-C & -0.111 & -0.178 \\
TG & -0.028 & -0.134 \\
HDL-C & 0.110 & -0.106 \\
Perceived Stress Score & & \\
Systolic Blood Pressure & -0.119 & -0.170 \\
Diastolic Blood Pressure & -0.156 & -0.132 \\
Fasting Blood Glucose & -0.066 & 0.118 \\
Total Cholesterol & -0.093 & -0.094 \\
LDL-C & -0.110 & -0.073 \\
TG & -0.065 & -0.006 \\
HDL-C & $\mathbf{0 . 2 1 0 *}$ & -0.167 \\
Family History of CHD & & \\
Systolic Blood Pressure & -0.021 & -0.162 \\
Diastolic Blood Pressure & 0.035 & -0.146 \\
Fasting Blood Glucose & -0.140 & 0.105 \\
Total Cholesterol & 0.198 & 0.148 \\
LDL-C & 0.161 & 0.054 \\
TG & 0.048 & 0.101 \\
HDL-C & 0.132 & \\
\hline
\end{tabular}

$* \mathrm{p}<0.05 ; * \mathrm{p}<0.01$

$\mathrm{CHD}=$ Coronary Heart Disease; LDL-C $=$ Low-density Lipoprotein Cholesterol; TG = Triglyceride; HDL-C = High-density Lipoprotein Cholesterol 
Table 55 - Correlations between Selected Socio-demographic Variables and the Physiological Risk Factors of the Participants by Ethnicity

\begin{tabular}{|c|c|c|c|}
\hline Variables & $\begin{array}{l}\text { FBCA } \\
\mathbf{n}=66\end{array}$ & $\begin{array}{l}\text { USBCA } \\
\mathrm{n}=62\end{array}$ & $\begin{array}{l}\mathbf{A A} \\
\mathbf{n}=61\end{array}$ \\
\hline \multicolumn{4}{|l|}{ Age } \\
\hline Systolic Blood Pressure & -0.045 & -0.087 & $0.500 * *$ \\
\hline Diastolic Blood Pressure & 0.054 & -0.116 & $0.406 * *$ \\
\hline Fasting Blood Glucose & -0.101 & -0.059 & 0.112 \\
\hline Total Cholesterol & 0.069 & -0.018 & 0.202 \\
\hline LDL-C & 0.054 & -0.010 & 0.153 \\
\hline TG & -0.148 & -0.080 & 0.032 \\
\hline $\mathrm{HDL}-\mathrm{C}$ & 0.170 & -0.099 & 0.064 \\
\hline \multicolumn{4}{|l|}{ Education } \\
\hline Systolic Blood Pressure & -0.078 & -0.066 & -0.012 \\
\hline Diastolic Blood Pressure & 0.047 & -0.112 & 0.080 \\
\hline Fasting Blood Glucose & 0.073 & 0.040 & -0.043 \\
\hline Total Cholesterol & 0.003 & $0.307 *$ & 0.247 \\
\hline LDL-C & -0.022 & $0.302 *$ & $0.309 *$ \\
\hline TG & -0.093 & 0.013 & 0.009 \\
\hline HDL-C & 0.083 & 0.124 & -0.119 \\
\hline \multicolumn{4}{|l|}{ Household Income } \\
\hline Systolic Blood Pressure & 0.046 & -0.089 & 0.090 \\
\hline Diastolic Blood Pressure & 0.081 & -0.079 & 0.083 \\
\hline Fasting Blood Glucose & -0.151 & 0.074 & 0.111 \\
\hline Total Cholesterol & $-0.297 *$ & -0.144 & 0.051 \\
\hline LDL-C & $-0.329 * *$ & -0.172 & 0.068 \\
\hline TG & -0.127 & -0.030 & -0.081 \\
\hline HDL-C & -0.019 & -0.012 & 0.158 \\
\hline \multicolumn{4}{|l|}{ Perceived Stress Score } \\
\hline Systolic Blood Pressure & -0.172 & $-0.377 * *$ & -0.008 \\
\hline Diastolic Blood Pressure & -0.109 & $-0.365 * *$ & -0.059 \\
\hline Fasting Blood Glucose & -0.145 & -0.057 & $0.284^{*}$ \\
\hline Total Cholesterol & -0.024 & -0.073 & -0.131 \\
\hline LDL-C & -0.070 & -0.062 & -0.134 \\
\hline TG & -0.075 & -0.039 & -0.005 \\
\hline $\mathrm{HDL}-\mathrm{C}$ & $0.267 *$ & -0.079 & -0.028 \\
\hline \multicolumn{4}{|l|}{ Family History of CHD } \\
\hline Systolic Blood Pressure & -0.193 & 0.009 & -0.103 \\
\hline Diastolic Blood Pressure & -0.160 & 0.041 & -0.065 \\
\hline Fasting Blood Glucose & -0.081 & -0.058 & -0.208 \\
\hline Total Cholesterol & 0.099 & $0.273^{*}$ & 0.025 \\
\hline LDL-C & 0.067 & $0.319 *$ & -0.005 \\
\hline $\mathrm{HDL}-\mathrm{C}$ & 0.055 & -0.114 & 0.123 \\
\hline
\end{tabular}

${ }^{*} \mathrm{p}<0.05 ;{ }^{* *} \mathrm{p}<0.01$

$\mathrm{CHD}=$ Coronary Heart Disease; LDL-C $=$ Low-density Lipoprotein Cholesterol; HDL-C $=$ Highdensity Lipoprotein Cholesterol

$\mathrm{FBCA}=$ Foreign-born Afro Caribbeans

USBCA = US-born Afro Caribbean Americans

$\mathrm{AA}=$ African Americans 


\section{Relationships between Behavioral/Environmental and Physiological Risk Factors}

The number of vegetable servings was negatively associated with $\mathrm{TC}(\mathrm{r}=0.231$; $\mathrm{p}<0.05$ ) in females (Table 56). In the male and female groups, there were strong and positive associations between $\mathrm{BMI}$ and $\mathrm{SBP}(\mathrm{r}=0.503, \mathrm{p}<0.01 ; \mathrm{r}=0.261 ; \mathrm{p}<0.01)$ and, BMI and DBP ( $r=0.471, p<0.01 ; r=0.309 ; p<0.01)$, respectively (Table 57). Also, in males, BMI was positively associated with TC $(r=0.293$; $<<0.01)$, LDL-C $(r=0.275 ; p<$ $0.01)$ and TG $(r=0.256 ; p<0.05)$, whereas in females, BMI was negatively associated with HDL-C $(r=-0.277 ; \mathrm{p}<0.01)$ (Table 57$)$. Cigarette smoking was positively associated with FBG $(r=0.202 ; \mathrm{p}<0.05)$ in females, whereas alcohol consumption was positively associated with triglyceride (TG) $(\mathrm{r}=0.253 ; \mathrm{p}<0.05)$ in males (Table 57$)$.

Within ethnic groups comparison showed the diet score was positively associated with HDL-C $(r=0.287 ; p<0.05)$ in the AA participants (Table 58). The number of vegetable servings was positively associated with $\mathrm{DBP}(\mathrm{r}=0.264 ; \mathrm{p}<0.05)$ and negatively with TC $(r=-0.325 ; \mathrm{p}<0.05)$ in USBCA participants. Also, in the USBCA participants, fiber intake was negatively associated with TC $(r=-0.306 ; p<0.05)$, whereas in AA participants dietary cholesterol intake was negatively associated with HDL-C $(r=-0.297$; $\mathrm{p}<0.05)($ Table 58). 
Table 56 - Correlations between Selected Dietary Components and Physiological Risk Factors of the Participants by Gender

\begin{tabular}{|c|c|c|}
\hline Variables & $\begin{array}{l}\text { Males } \\
\mathrm{n}=89\end{array}$ & $\begin{array}{l}\text { Females } \\
\mathbf{n}=96\end{array}$ \\
\hline \multicolumn{3}{|l|}{ Diet Score } \\
\hline Systolic BP & -0.083 & -0.145 \\
\hline Diastolic BP & -0.081 & -0.164 \\
\hline Fasting BG & -0.108 & -0.018 \\
\hline Total Cholesterol & 0.187 & 0.001 \\
\hline LDL-C & 0.190 & 0.007 \\
\hline TG & -0.041 & 0.040 \\
\hline HDL-C & 0.093 & -0.083 \\
\hline \multicolumn{3}{|l|}{ Vegetable Servings } \\
\hline Systolic BP & 0.165 & 0.110 \\
\hline Diastolic BP & 0.031 & 0.133 \\
\hline Fasting BG & -0.017 & 0.183 \\
\hline Total Cholesterol & 0.009 & $-0.231^{*}$ \\
\hline LDL-C & 0.030 & -0.192 \\
\hline TG & -0.069 & -0.025 \\
\hline HDL-C & -0.010 & 0.009 \\
\hline \multicolumn{3}{|l|}{ Fiber } \\
\hline Body Mass Index & 0.086 & 0.011 \\
\hline Systolic BP & 0.145 & 0.018 \\
\hline Diastolic BP & 0.004 & 0.063 \\
\hline Fasting BG & -0.002 & 0.071 \\
\hline Total Cholesterol & -0.057 & -0.162 \\
\hline LDL-C & -0.062 & -0.100 \\
\hline TG & -0.047 & 0.032 \\
\hline HDL-C & 0.133 & -0.068 \\
\hline \multicolumn{3}{|l|}{ Cholesterol } \\
\hline Body Mass Index & 0.013 & -0.101 \\
\hline Systolic BP & -0.032 & -0.002 \\
\hline Diastolic BP & -0.067 & -0.078 \\
\hline Fasting BG & -0.070 & -0.046 \\
\hline Total Cholesterol & 0.199 & -0.064 \\
\hline LDL-C & 0.190 & -0.045 \\
\hline $\mathrm{TG}$ & 0.018 & -0.079 \\
\hline HDL-C & 0.073 & -0.032 \\
\hline
\end{tabular}

${ }^{*} \mathrm{p}<0.05$

$\mathrm{BP}=$ Blood Pressure BG = Blood Glucose LDL-C = Low-density Lipoprotein Cholesterol; TG

$=$ Triglyceride; HDL-C $=$ High-density Lipoprotein Cholesterol 
Table 57 - Correlations between the Behavioral/Environmental and Physiological Risk Factors of the Participants by Gender

\begin{tabular}{lcc}
\hline Variables & $\begin{array}{l}\text { Males } \\
\mathbf{n}=\mathbf{8 9}\end{array}$ & $\begin{array}{c}\text { Females } \\
\mathbf{n}=\mathbf{9 6}\end{array}$ \\
\hline Physical Activity Index & & \\
Systolic BP & 0.091 & -0.013 \\
Diastolic BP & 0.018 & -0.013 \\
Fasting BG & 0.085 & 0.018 \\
Total Cholesterol & 0.008 & -0.112 \\
LDL-C & 0.041 & -0.138 \\
TG & -0.059 & 0.024 \\
HDL-C & -0.045 & 0.163 \\
Body Mass Index & & \\
Systolic BP & $\mathbf{0 . 5 0 3 * *}$ & $\mathbf{0 . 2 6 1 ^ { * * }}$ \\
Diastolic BP & $\mathbf{0 . 4 7 1 ^ { * * }}$ & $\mathbf{0 . 3 0 9}$ \\
Fasting BG & 0.156 & 0.075 \\
Total Cholesterol & $\mathbf{0 . 2 9 3 ^ { * * }}$ & -0.059 \\
LDL-C & $\mathbf{0 . 2 7 5 ^ { * * }}$ & -0.002 \\
TG & $\mathbf{0 . 2 5 6 ^ { * }}$ & 0.088 \\
HDL-C & -0.140 & $\mathbf{- 0 . 2 7 7 ^ { * * }}$ \\
Cigarette Smoking & & \\
Body Mass Index & 0.077 & -0.107 \\
Systolic BP & -0.114 & 0.149 \\
Diastolic BP & -0.158 & 0.063 \\
Fasting BG & -0.057 & $\mathbf{0 . 2 0 2}$ \\
Total Cholesterol & 0.108 & -0.005 \\
LDL-C & 0.205 & -0.110 \\
TG & 0.085 & 0.124 \\
HDL-C & -0.158 & -0.053 \\
Alcohol Intake & & \\
Systolic BP & -0.158 & 0.044 \\
Diastolic BP & 0.002 & -0.060 \\
Fasting BG & 0.031 & -0.016 \\
Total Cholesterol & 0.102 & 0.019 \\
LDL-C & 0.054 & 0.065 \\
TG & $\mathbf{0 . 2 5 3 ^ { * }}$ & -0.034 \\
HDL-C & -0.140 & \\
& & \\
\hline
\end{tabular}

${ }^{*} \mathrm{p}<0.05 ;{ }^{*} \mathrm{p}<0.01$

$\mathrm{BP}=$ Blood Pressure; $\mathrm{BG}=$ Blood Glucose; LDL-C = Low-density Lipoprotein Cholesterol; TG

$=$ Triglyceride; HDL-C $=$, High-density Lipoprotein Cholesterol 
Table 58 - Correlations between Selected Dietary Components and Physiological Risk Factors of the Participants by Ethnicity

\begin{tabular}{|c|c|c|c|}
\hline Variables & $\begin{array}{l}\text { FBCA } \\
\mathrm{n}=65\end{array}$ & $\begin{array}{l}\text { USBCA } \\
\mathrm{n}=61\end{array}$ & $\begin{array}{l}\mathrm{AA} \\
\mathrm{n}=59\end{array}$ \\
\hline \multicolumn{4}{|l|}{ Diet Score } \\
\hline Systolic BP & -0.050 & 0.048 & 0.015 \\
\hline Diastolic BP & -0.013 & 0.087 & -0.144 \\
\hline Fasting BG & -0.091 & 0.043 & -0.052 \\
\hline Total Cholesterol & -0.071 & 0.201 & -0.103 \\
\hline LDL-C & -0.081 & 0.234 & 0.013 \\
\hline TG & 0.203 & -0.095 & -0.124 \\
\hline HDL-C & -0.181 & -0.056 & $0.287^{*}$ \\
\hline \multicolumn{4}{|l|}{ Vegetable Servings } \\
\hline Systolic BP & 0.091 & 0.187 & 0.161 \\
\hline Diastolic BP & -0.065 & $0.264^{*}$ & 0.077 \\
\hline Fasting BG & 0.011 & 0.146 & 0.143 \\
\hline Total Cholesterol & -0.038 & $-0.325^{*}$ & 0.063 \\
\hline LDL-C & -0.012 & -0.210 & 0.004 \\
\hline TG & -0.237 & -0.111 & 0.229 \\
\hline HDL-C & 0.057 & -0.144 & 0.015 \\
\hline \multicolumn{4}{|l|}{ Fiber } \\
\hline Systolic BP & 0.139 & 0.037 & 0.172 \\
\hline Diastolic BP & -0.128 & 0.246 & 0.109 \\
\hline Fasting BG & -0.014 & 0.202 & -0.003 \\
\hline Total Cholesterol & -0.077 & $-0.306^{*}$ & 0.038 \\
\hline LDL-C & -0.055 & -0.197 & 0.024 \\
\hline TG & -0.131 & 0.013 & 0.146 \\
\hline HDL-C & 0.075 & -0.187 & -0.062 \\
\hline \multicolumn{4}{|l|}{ Cholesterol } \\
\hline Systolic BP & 0.059 & 0.078 & 0.183 \\
\hline Diastolic BP & -0.118 & 0.119 & 0.114 \\
\hline Fasting BG & -0.097 & 0.075 & -0.065 \\
\hline Total Cholesterol & 0.016 & 0.088 & -0.093 \\
\hline LDL-C & -0.021 & 0.157 & 0.000 \\
\hline TG & 0.154 & -0.129 & -0.018 \\
\hline HDL-C & -0.147 & -0.129 & $-0.297 *$ \\
\hline
\end{tabular}

${ }^{*} \mathrm{p}<0.05$.

BP = Blood Pressure; BG = Blood Glucose; LDL-C = Low-density Lipoprotein Cholesterol; TG

$=$ Triglyceride; HDL-C $=$ High-density Lipoprotein Cholesterol

FBCA $=$ Foreign-born Afro Caribbeans

USBCA = US-born Afro Caribbean Americans

$\mathrm{AA}=$ African Americans 
For the AA participants, the physical activity index (PAI) was positively associated with SBP $(r=0.312 ; \mathrm{p}<0.05)$, whereas for the USBCA participants, the PAI was negatively associated with TC $(r=-0.319 ; p<0.05)$ and LDL-C $(r=-0.329 ; p<0.01)$ (Table 59). For the FBCA participants, BMI was positively correlated with DBP $(r=0.270 ; p<0.05)$, whereas for the USBCA and AA participants, BMI was strongly and positively associated with $\operatorname{SBP}(\mathrm{r}=0.514, \mathrm{p}<0.01 ; \mathrm{r}=0.566 ; \mathrm{p}<0.01)$ and $\mathrm{DBP}(\mathrm{r}=0.434$, $\mathrm{p}<0.01 ; \mathrm{r}=0.555 ; \mathrm{p}<0.01)$, respectively. The BMI was also associated positively with TC $(r=0.270 ; \mathrm{p}<0.05)$ and LDL-C $(r=0.307 ; \mathrm{p}<0.05)$ in the USBCA participants, whereas the BMI was negatively associated with HDL-C in the FBCA $(r=-0.309 ; \mathrm{p}<0.05)$ and AA participants $(r=-0.306 ; \mathrm{p}<0.05)($ Table 59).

\section{Relationships between Socio-demographic Variables and Emerging Risk Factors}

Within gender groups comparison showed no significant relationship between the socio-demographic variables and the emerging risk factors for $\mathrm{CHD}$ (Table 60). Within ethnic groups comparison showed age was positively associated with TC/HDL-C ratio $(r=0.282 ; \mathrm{p}<0.05)$ in AA participants (Table 60). For the AA participants, education was positively associated with TC/HDL-C ratio $(r=0.289 ; \mathrm{p}<0.05)$, whereas for the USBCA participants, family history of $\mathrm{CHD}$ was positively associated with hs-CRP ( $\mathrm{r}=0.270$; $\mathrm{p}<$ 0.05) (Table 60). 
Table 59 - Correlations between Behavioral/Environmental and Physiological Risk Factors of the Participants by Ethnicity

\begin{tabular}{|c|c|c|c|}
\hline Variables & $\begin{array}{l}\text { FBCA } \\
n=66\end{array}$ & $\begin{array}{l}\text { USBCA } \\
\mathrm{n}=62\end{array}$ & $\begin{array}{l}\mathrm{AA} \\
\mathrm{n}=61\end{array}$ \\
\hline \multicolumn{4}{|c|}{ Physical Activity Index } \\
\hline Systolic BP & 0.044 & 0.121 & $0.312^{*}$ \\
\hline Diastolic BP & -0.067 & 0.196 & 0.229 \\
\hline Fasting BG & 0.096 & 0.030 & 0.058 \\
\hline Total Cholesterol & -0.118 & $-0.319^{*}$ & 0.143 \\
\hline LDL-C & -0.058 & $-0.329 * *$ & 0.219 \\
\hline TG & 0.125 & -0.211 & 0.052 \\
\hline HDL-C & -0.232 & 0.079 & -0.226 \\
\hline \multicolumn{4}{|l|}{ Body Mass Index } \\
\hline SBP & 0.114 & $0.514^{* *}$ & $0.566^{* *}$ \\
\hline DBP & $0.270^{*}$ & $0.434^{* *}$ & $0.555^{* *}$ \\
\hline FBG & 0.172 & 0.151 & 0.088 \\
\hline $\mathrm{TC}$ & 0.041 & $0.270^{*}$ & -0.036 \\
\hline LDL-C & 0.111 & $0.307 *$ & -0.002 \\
\hline TG & 0.126 & 0.157 & 0.241 \\
\hline HDL-C & $-0.309^{*}$ & -0.219 & $-0.306^{*}$ \\
\hline \multicolumn{4}{|l|}{ Cigarette Smoking } \\
\hline Systolic BP & -- & 0.084 & 0.020 \\
\hline Diastolic BP & -- & 0.046 & -0.033 \\
\hline Fasting BG & -- & 0.072 & 0.136 \\
\hline Total Cholesterol & -- & 0.177 & 0.082 \\
\hline LDL-C & -- & 0.209 & 0.009 \\
\hline TG & -- & 0.081 & 0.049 \\
\hline HDL-C & -- & -0.121 & 0.206 \\
\hline \multicolumn{4}{|l|}{ Alcohol Intake } \\
\hline Systolic BP & -0.094 & -0.137 & 0.049 \\
\hline Diastolic BP & -0.005 & 0.016 & 0.067 \\
\hline Fasting $B G$ & 0.004 & 0.002 & -0.092 \\
\hline Total Cholesterol & 0.114 & 0.006 & 0.085 \\
\hline LDL-C & 0.070 & -0.009 & 0.098 \\
\hline TG & 0.147 & 0.078 & 0.214 \\
\hline HDL-C & 0.224 & -0.089 & -0.181 \\
\hline
\end{tabular}

$* \mathrm{p}<0.05 ; * * \mathrm{p}<0.01$

BP = Blood Pressure; BG = Blood Glucose; LDL-C = Low-density Lipoprotein Cholesterol; TG = Triglyceride; HDL-C $=$ High-density Lipoprotein Cholesterol

FBCA = Foreign-born Afro Caribbeans

USBCA = US-born Afro Caribbean Americans

$\mathrm{AA}=$ African Americans 
Table 60 - Correlations between selected Socio-demographic Variables and the Emerging Risk Factors of the Participants by Gender and by Ethnicity

\begin{tabular}{|c|c|c|c|}
\hline $\begin{array}{l}\text { Gender } \\
\text { Variables }\end{array}$ & & & $\begin{array}{c}\text { Females } \\
\mathbf{n}=98\end{array}$ \\
\hline \multicolumn{4}{|l|}{ Age } \\
\hline hs-CRP & \multicolumn{2}{|c|}{0.089} & 0.115 \\
\hline TC/HDL-C Ratio & \multicolumn{2}{|c|}{0.135} & 0.071 \\
\hline \multicolumn{4}{|l|}{ Education } \\
\hline hs-CRP & \multicolumn{2}{|c|}{-0.077} & 0.088 \\
\hline TC/HDL-C Ratio & \multicolumn{2}{|c|}{0.046} & 0.079 \\
\hline \multicolumn{4}{|l|}{ Household Income } \\
\hline hs-CRP & \multirow{2}{*}{\multicolumn{2}{|c|}{$\begin{array}{l}-0.017 \\
-0.005\end{array}$}} & \multirow{2}{*}{$\begin{array}{r}0.065 \\
-0.112\end{array}$} \\
\hline TC/HDL-C Ratio & & & \\
\hline \multicolumn{4}{|c|}{ Family History of CHD } \\
\hline hs-CRP & \multicolumn{2}{|c|}{0.153} & 0.107 \\
\hline TC/HDL-C Ratio & \multicolumn{2}{|c|}{0.042} & 0.136 \\
\hline $\begin{array}{l}\text { Ethnicity } \\
\text { Variables }\end{array}$ & $\begin{array}{c}\begin{array}{c}\text { FBCA } \\
n=66\end{array} \\
\text { n }\end{array}$ & $\begin{array}{c}\text { USBCA } \\
n=62\end{array}$ & $\begin{array}{c}\mathbf{A A} \\
\mathrm{n}=61\end{array}$ \\
\hline \multicolumn{4}{|l|}{ Age } \\
\hline hs-CRP & 0.070 & 0.106 & 0.076 \\
\hline TC/HDL-C Ratio & 0.113 & 0.097 & $0.282^{*}$ \\
\hline \multicolumn{4}{|l|}{ Education } \\
\hline hs-CRP & 0.155 & 0.087 & -0.131 \\
\hline TC/HDL-C Ratio & -0.105 & 0.103 & $0.289 *$ \\
\hline \multicolumn{4}{|l|}{ Household Income } \\
\hline hs-CRP & 0.128 & -0.016 & 0.074 \\
\hline TC/HDL-C Ratio & -0.172 & -0.049 & 0.048 \\
\hline \multicolumn{4}{|l|}{ Family History of } \\
\hline \multicolumn{4}{|l|}{ CHD } \\
\hline hs-CRP & -0.015 & $0.270^{*}$ & 0.160 \\
\hline TC/HDL-C Ratio & 0.065 & 0.158 & -0.082 \\
\hline
\end{tabular}

\footnotetext{
* $\mathrm{p}<0.05$
}

$\mathrm{CHD}=$ Coronary Heart Disease; hs-CRP = high-sensitivity C-reactive Protein, TC/HDL-C $=$ Total Cholesterol to High-density Lipoprotein Cholesterol

FBCA = Foreign-born Afro Caribbeans

USBCA = US-born Afro Caribbean Americans

$\mathrm{AA}=$ African Americans 


\section{Relationships between Behavioral/Environmental and Emerging Risk Factors}

The number of vegetable servings was negatively associated with TC/HDL-C ratio $(\mathrm{r}=-0.201 ; \mathrm{p}<0.05)$ in females (Table 61$)$. In males and females, BMI was positively associated with hs-CRP $(r=0.258, p<0.05 ; r=0.377, p<0.01)$, whereas in males only, BMI was positively associated with TC/HDL-C ratio $(r=0.325 ; \mathrm{p}<0.01)$ (Table 62). Also, cigarette smoking was positively associated with hs-CRP $(r=0.397 ; p<0.01)$ in males (Table 62). Within ethnic groups comparison showed the PAI was negatively associated with TC/HDL-C ratio $(r=-0.340 ; p<0.01)$ in USBCA participants (Table 62). For the FBCA and USBCA participants, BMI was positively associated with hs-CRP ( $\mathrm{r}=0.278$, $\mathrm{p}<0.05 ; \mathrm{r}=0.357, \mathrm{p}<0.05)$ and TC/HDL-C ratio $(\mathrm{r}=0.287, \mathrm{p}<0.05 ; \mathrm{r}=0.298, \mathrm{p}<0.05)$, respectively, whereas $\mathrm{BMI}$ was only positively associated with hs-CRP $(\mathrm{r}=0.308 ; \mathrm{p}<$ 0.05 ) in the AA participants (Table 62). Also, cigarette smoking was strongly and positively associated with hs-CRP $(r=0.522 ; \mathrm{p}<0.01)$ in USBCA participants (Table 62). 
Table 61 - Correlations between Selected Dietary Components and the Emerging Risk Factors of the Participants by Gender and by Ethnicity

\begin{tabular}{|c|c|c|c|}
\hline $\begin{array}{l}\text { Gender } \\
\text { Variables }\end{array}$ & \multicolumn{2}{|c|}{$\begin{array}{l}\text { Males } \\
\mathrm{n}=91\end{array}$} & $\begin{array}{c}\begin{array}{c}\text { Females } \\
\mathbf{n}=98\end{array} \\
\end{array}$ \\
\hline \multicolumn{4}{|l|}{ Diet Score } \\
\hline hs-CRP & \multirow{2}{*}{\multicolumn{2}{|c|}{$\begin{array}{r}-0.051 \\
0.073\end{array}$}} & 0.041 \\
\hline TC/HDL-C Ratio & & & 0.053 \\
\hline \multicolumn{4}{|l|}{ Vegetable Servings } \\
\hline hs-CRP & \multirow{2}{*}{\multicolumn{2}{|c|}{$\begin{array}{r}0.211 \\
-0.006\end{array}$}} & -0.047 \\
\hline TC/HDL-C Ratio & & & $-0.201 *$ \\
\hline \multicolumn{4}{|l|}{ Fiber } \\
\hline hs-CRP & \multicolumn{2}{|c|}{0.063} & -0.037 \\
\hline TC/HDL-C Ratio & \multicolumn{2}{|c|}{-0.123} & -0.105 \\
\hline Homocysteine & \multicolumn{2}{|c|}{0.129} & 0.182 \\
\hline \multicolumn{4}{|l|}{ Cholesterol } \\
\hline hs-CRP & \multicolumn{2}{|c|}{0.030} & -0.068 \\
\hline TC/HDL-C Ratio & \multicolumn{2}{|c|}{0.090} & -0.057 \\
\hline Ethnicity & FBCA & USBCA & $\mathbf{A A}$ \\
\hline Variables & $\mathrm{n}=66$ & $n=62$ & $\mathrm{n}=61$ \\
\hline \multicolumn{4}{|l|}{ Diet Score } \\
\hline hs-CRP & 0.150 & 0.049 & -0.214 \\
\hline TC/HDL-C Ratio & 0.056 & 0.138 & 0.146 \\
\hline \multicolumn{4}{|l|}{ Vegetable Servings } \\
\hline hs-CRP & 0.023 & 0.036 & -0.018 \\
\hline TC/HDL-C Ratio & -0.072 & -0.202 & 0.063 \\
\hline \multicolumn{4}{|l|}{ Fiber } \\
\hline hs-CRP & -0.004 & -0.025 & -0.091 \\
\hline TC/HDL-C Ratio & -0.111 & -0.170 & 0.087 \\
\hline \multicolumn{4}{|l|}{ Cholesterol } \\
\hline hs-CRP & -0.072 & -0.126 & -0.074 \\
\hline TC/HDL-C Ratio & 0.046 & 0.083 & 0.181 \\
\hline
\end{tabular}

\section{$* \mathrm{p}<0.05$}

hs-CRP = high-sensitivity $\mathrm{C}$-reactive Protein, TC/HDL-C = Total Cholesterol to High-density Lipoprotein Cholesterol FBCA = Foreign-born Afro Caribbeans USBCA $=$ US-born Afro Caribbean Americans $\mathrm{AA}=$ African Americans 
Table 62 - Correlations between the Behavioral/Environmental and the Emerging Risk Factors of the Participants by Gender and Ethnicity

\begin{tabular}{|c|c|c|c|}
\hline & \multicolumn{2}{|c|}{$\begin{array}{c}\text { Males } \\
\mathrm{n}=91\end{array}$} & $\begin{array}{c}\text { Females } \\
\mathbf{n}=98\end{array}$ \\
\hline Variables & \multicolumn{2}{|c|}{ rvalue } & \\
\hline \multicolumn{4}{|l|}{ Physical Activity Index } \\
\hline hs-CRP & \multicolumn{2}{|c|}{-0.039} & -0.022 \\
\hline TC/HDL-C Ratio & \multicolumn{2}{|c|}{0.055} & $-0.232^{*}$ \\
\hline \multicolumn{4}{|l|}{ Body Mass Index } \\
\hline hs-CRP & \multicolumn{2}{|c|}{$0.258 *$} & $0.377^{* *}$ \\
\hline TC/HDL-C Ratio & \multicolumn{2}{|c|}{$0.325^{* *}$} & 0.136 \\
\hline \multicolumn{4}{|l|}{ Cigarette Smoking } \\
\hline hs-CRP & \multirow{2}{*}{\multicolumn{2}{|c|}{$\begin{array}{l}0.397 * * \\
0.121\end{array}$}} & -0.102 \\
\hline TC/HDL-C Ratio & & & -0.066 \\
\hline \multicolumn{4}{|l|}{ Alcohol Intake } \\
\hline hs-CRP & \multicolumn{2}{|c|}{0.059} & 0.129 \\
\hline TC/HDL-C Ratio & \multicolumn{2}{|c|}{0.090} & 0.034 \\
\hline By Ethnicity & $\begin{array}{c}\mathrm{FBCA} \\
\mathrm{n}=66\end{array}$ & $\begin{array}{c}\begin{array}{c}\text { USBCA } \\
\mathrm{n}=62\end{array} \\
\end{array}$ & $\begin{array}{c}\mathbf{A A} \\
\mathrm{n}=61\end{array}$ \\
\hline \multicolumn{4}{|l|}{ Physical Activity Index } \\
\hline hs-CRP & -0.060 & -0.141 & -0.112 \\
\hline TC/HDL-C Ratio & 0.076 & $-0.340^{* *}$ & -0.141 \\
\hline \multicolumn{4}{|l|}{ Body Mass Index } \\
\hline hs-CRP & $0.278^{*}$ & $0.357 *$ & $0.308^{*}$ \\
\hline TC/HDL-C Ratio & $0.287 *$ & $0.298 *$ & 0.239 \\
\hline \multicolumn{4}{|l|}{ Cigarette Smoking } \\
\hline hs-CRP & - & $0.522^{* *}$ & -0.010 \\
\hline TC/HDL-C Ratio & -- & 0.137 & -0.079 \\
\hline \multicolumn{4}{|l|}{ Alcohol Intake } \\
\hline hs-CRP & -0.231 & 0.189 & 0.241 \\
\hline TC/HDL-C Ratio & -0.043 & 0.058 & 0.233 \\
\hline $\begin{array}{l}{ }^{*} \mathrm{p}<0.05 ;{ }^{*} \mathrm{p}<0.01 \\
\text { hs-CRP, high-sensitivit } \\
\text { Lipoprotein Cholestero } \\
\text { FBCA = Foreign-born A } \\
\text { USBCA = US-born Afro } \\
\text { AA = African American }\end{array}$ & Ams & al Chole & High-density \\
\hline
\end{tabular}




\section{Relationships between Bio-physiological and Emerging Risk Factors}

In females, the DBP $(r=0.329 ; \mathrm{p}<0.01)$ and the SBP $(\mathrm{r}=0.257 ; \mathrm{p}<0.05)$ were positively associated with hs-CRP, whereas in males, DBP was positively associated with TC/HDL-C ratio $(r=0.257 ; \mathrm{p}<0.05)$ (Table 63). In both gender groups, $\mathrm{TC}(\mathrm{r}=0.797, \mathrm{p}<$ $0.001 ; \mathrm{r}=0.676, \mathrm{p}<0.001)$ and LDL-C $(r=0.843, \mathrm{p}<0.001 ; \mathrm{r}=0.809, \mathrm{p}<0.001)$, respectively were strongly and positively associated with TC/HDL-C ratio. In males, serum triglyceride (TG) was positively associated with hs-CRP $(r=0.250 ; p<0.05)$ and TC/HDL-C ratio $(r=0.471 ; p<0.01)$. In both male and female groups, high HDL-C levels were negatively associated with TC/HDL-C ratio $(r=-0.480, p<0.01 ; \mathrm{r}=-0.525, \mathrm{p}<0.01)$, respectively (Table 63).

For the FBCA, USBCA and AA participants, the TC and LDL-C levels correlated strongly and positively with the TC/HDL-C ratio $(r=0.699, \mathrm{p}<0.001 ; \mathrm{r}=0.703, \mathrm{p}<0.001$; $\mathrm{r}=0.627, \mathrm{p}<0.001$ ), respectively (Table 64). Also, for the USBCA participants, serum TG was positively associated with TC/HDL-C ratio $(r=0.421 ; \mathrm{p}<0.01)$. For the $\mathrm{FBCA}$, USBCA and AA participants, HDL-C levels were negatively associated with TC/HDL-C ratio $(\mathrm{r}=-0.582, \mathrm{p}<0.01 ; \mathrm{r}=-0.548, \mathrm{p}<0.01 ; \mathrm{r}=-0.490, \mathrm{p}<0.01)$, respectively (Table 64). 
Table 63 - Correlations between the Physiological and the Emerging Risk Factors of the Participant by Gender

\begin{tabular}{|c|c|c|}
\hline Variables & $\begin{array}{r}\text { Males } \\
\mathrm{n}=91 \\
\end{array}$ & $\begin{array}{l}\text { Females } \\
\mathrm{n}=98\end{array}$ \\
\hline \multicolumn{3}{|c|}{ Systolic Blood Pressure } \\
\hline hs-CRP & 0.063 & $0.257 *$ \\
\hline TC/HDL-C Ratio & 0.178 & -0.193 \\
\hline \multicolumn{3}{|c|}{ Diastolic Blood Pressure } \\
\hline hs-CRP & 0.143 & $0.329 * *$ \\
\hline TC/HDL-C Ratio & $0.257^{*}$ & -0.182 \\
\hline \multicolumn{3}{|l|}{ Fasting Blood Glucose } \\
\hline hs-CRP & 0.041 & -0.069 \\
\hline TC/HDL-C Ratio & -0.009 & -0.038 \\
\hline \multicolumn{3}{|l|}{ Total Cholesterol } \\
\hline hs-CRP & 0.131 & -0.088 \\
\hline TC/HDL-C Ratio & $0.797 * * *$ & $0.676 * * *$ \\
\hline Homocysteine & 0.096 & -0.060 \\
\hline \multicolumn{3}{|l|}{ LDL-C } \\
\hline hs-CRP & 0.112 & -0.097 \\
\hline TC/HDL-C Ratio & $0.843 * * *$ & $0.809 * * *$ \\
\hline \multicolumn{3}{|l|}{ Triglyceride } \\
\hline hs-CRP & $0.250^{*}$ & -0.047 \\
\hline TC/HDL-C Ratio & $0.471^{* *}$ & 0.110 \\
\hline \multicolumn{3}{|l|}{ HDL-C } \\
\hline hs-CRP & -0.189 & -0.027 \\
\hline TC/HDL-C Ratio & $-0.480 * *$ & $-0.525^{* *}$ \\
\hline
\end{tabular}

${ }^{*} \mathrm{p}<0.05 ;{ }^{* *} \mathrm{p}<0.01 ; * * * \mathrm{p}<0.001$

hs-CRP = high-sensitivity C-reactive Protein, TC/HDL-C = Total Cholesterol to High-density Lipoprotein Cholesterol 
Table 64 - Correlations between the Physiological and the Emerging Risk Factors of the Participant by Ethnicity

\begin{tabular}{|c|c|c|c|}
\hline Variables & $\begin{array}{c}\mathrm{FBCA} \\
\mathbf{n}=31 \\
\end{array}$ & $\begin{array}{l}\text { USBCA } \\
\mathrm{n}=30\end{array}$ & $\begin{array}{l}\mathrm{AA} \\
\mathrm{n}=30\end{array}$ \\
\hline \multicolumn{4}{|c|}{ Systolic Blood Presssure } \\
\hline hs-CRP & 0.123 & 0.170 & 0.027 \\
\hline TC/HDL-C Ratio & 0.003 & -0.008 & 0.220 \\
\hline \multicolumn{4}{|c|}{ Diastolic Blood Pressure } \\
\hline hs-CRP & 0.165 & 0.225 & 0.224 \\
\hline TC/HDL-C Ratio & 0.139 & -0.082 & 0.216 \\
\hline \multicolumn{4}{|l|}{ Fasting Blood Glucose } \\
\hline hs-CRP & -0.064 & 0.047 & -0.129 \\
\hline TC/HDL-C Ratio & 0.132 & -0.139 & -0.051 \\
\hline \multicolumn{4}{|l|}{ Total Cholesterol } \\
\hline hs-CRP & -0.184 & 0.235 & -0.137 \\
\hline TC/HDL-C Ratio & $0.699 * * *$ & $0.703 * * *$ & $0.627 * * *$ \\
\hline \multicolumn{4}{|l|}{ LDL-C } \\
\hline hs-CRP & -0.214 & 0.223 & -0.155 \\
\hline TC/HDL-C Ratio & $0.805 * * *$ & $0.801 * *$ & $0.797 * * *$ \\
\hline \multicolumn{4}{|l|}{ Triglyceride } \\
\hline hs-CRP & -0.191 & 0.139 & 0.177 \\
\hline TC/HDL-C Ratio & 0.197 & $0.421 * *$ & 0.219 \\
\hline \multicolumn{4}{|l|}{ HDL-C } \\
\hline hs-CRP & 0.182 & 0.013 & -0.130 \\
\hline TC/HDL-C Ratio & $-0.582 * *$ & $-0.548 * *$ & $-0.490 * *$ \\
\hline
\end{tabular}

$* \mathrm{p}<0.05 ; * * \mathrm{p}<0.01 ; * * * \mathrm{p}<0.001$

hs-CRP = high-sensitivity C-reactive Protein, TC/HDL-C $=$ Total Cholesterol to High-density Lipoprotein Cholesterol FBCA = Foreign-born Afro Caribbeans USBCA = US-born Afro Caribbean Americans $\mathrm{AA}=$ African Americans 


\section{Research Questions Summary}

Research Question 1 - Are the socio-demographic profiles of FBCA, USBCA and AA adults similar?

The mean age of USBCA participants was significant $(p<0.01)$ lower than the mean age of the AA participants but not the FBCA participants. Also, the mean age of the USBCA males was significantly $(p<0.01)$ lower than the mean age of the AA males but not the FBCA males. Significantly $(\mathrm{p}<0.05)$ more USBCA $(96.8 \%)$ participants were attending school compared to FBCA (83.4\%) and AA (73.8\%) participants. However, school attendance was significantly $(p<0.05)$ higher in the USBCA $(96.7 \%)$ males compared to the AA (60.0\%) males but not the FBCA (77.4\%) males. However, no significance differences between the three female ethnic subgroups with respect to the socio-demographic variables were observed

Research Question 2 -Are FBCA adults less likely to have behavioral/environmental risk factors for CHD as compared to USBCA and AA adults?

The behavioral/environmental risk factors for CHD were significantly $(\mathrm{p}<0.05)$ lower in FBCA participants compared to the USBCA and AA participants. The FBCA participants compared to the USBCA and AA participants had higher quality of dietary intakes (diet score $3.5 \pm 1.6$ vs. $2.7 \pm 1.7$ and $2.6 \pm 1.5$, range $0-7$, where $0=$ 'poor' diet quality and 7 = 'good' diet quality), were less likely to watch television often/very often ( $45.5 \%$ vs. $54.8 \%$ and $49.2 \%$ ), were less obese ( $7.6 \%$ vs. $17.7 \%$ and $23.0 \%$ ), did not smoke cigarettes ( $0.0 \%$ vs. $4.8 \%$ and $6.6 \%$ ) and consumed less alcoholic beverages 
( $24.2 \%$ vs. $29.0 \%$ and $50.8 \%)$, respectively. Significantly $(p<0.05)$ more FBCA participants than USBCA and AA participants met Dietary Guidelines for percent energy from fat, whereas significantly $(p<0.05)$ more FBCA participants compared to AA but not USBCA participants met the Dietary and Food Guide Pyramid Guidelines for percent energy from carbohydrate and servings of fruits, respectively.

Additionally, significantly $(\mathrm{p}<0.05)$ more USBCA (22.6\%) and AA (19.7\%) participants compared to FBCA participants $(6.1 \%)$ had three or more combined behavioral/environmental risk factors for CHD.

Research Question 3 - Are FBCA adults less likely to have physiological risk factors for CHD as compared to USBCA and AA adults?

In general, there were no statistical significant differences between the physiological risk factors for CHD of the FBCA, USBCA and AA participants.

Research Question 4 - Are FBCA adults less likely to have emerging risk factors for CHD as compared to USBCA and AA adults?

The FBCA and USBCA participants compared to the AA participants had significantly $(\mathrm{p}<0.01)$ lower mean hs-CRP levels $(1.1 \pm 1.3$ and $1.3 \pm 1.6$ vs. $2.2 \pm 2.7)$, respectively. In general, significant differences were observed between males and females. The significant differences observed within the ethnic groups were also observed more frequently within the male ethnic subgroups than within the female ethnic subgroups. 


\section{CHAPTER V}

\section{DISCUSSION}

This study investigated the relationship between risk factors for coronary heart disease (CHD) in three ethnic groups of a predominantly Black population in South Florida. The study addressed the question of (keeping race and geography constant) whether there are differences in (a) socio-demographic (b) behavioral/environmental (c) bio-physiological and, (d) emerging risk factors for CHD between apparently healthy foreign-born Afro Caribbean American (FBCA) living in the US for less than 10 years, US-born Afro Caribbean American (USBCA) and African American (AA) adults (18-40 years), living in South Florida. This is the first comprehensive study of its kind presenting socio-demographic, behavioral, bio-physiological and emerging risk profiles for FBCA and USBCA in South Florida.

Research Question 1: Are the socio-demographic profiles of FBCA, USBCA and AA adults similar?

\section{Socio-economic status and Perceived Stress}

The risk for CHD in males and females increases with advancing age (NCEP: ATP III, 2001). In our study, the AA participants were significantly older than the USBCA but not the FBCA participants. Age was positively associated with the diet score $(r=0.245 ; \mathrm{p}<0.05)$ in males and FBCA participants $(r=0.321 ; \mathrm{p}<0.05)$ an indication that older individuals had better diet practices. 
There is evidence indicating that individuals with lower socio-economic status (SES), using income and educational levels as proxies for SES, have a higher risk for stroke and CHD compared to those in the higher SES groups (AHA Statistical Update, 2002; Kaplan and Keil, 1993). Individuals from lower socio-economic groups also have poorer health habits and health knowledge (Wood et al., 1998).

In our study, the reported socio-demographic variables of the FBCA, USBCA and AA participants showed differences. However, these differences did not reach significance possibly due to small sample sizes and non-randomized study design. In our study, although more AA (71\%) and USBCA (82\%) compared to FBCA (67\%) participants reported completing high school, more FBCA (33\%) compared to AA (29\%) and USBCA (18\%) participants were in graduate school. This is in contrast to the report of Harris (1999) that showed foreign-born adults (64.5\%) were less educated (completed 12 years or more of school) than US-born (78.7\%) adults. Our study is supported by the reports of Fang et al (1996), Singh and Siahpush (2002) and Logan and Deane (2003) who used larger sample sizes. Fang et al (1996) reported that $77.9 \%$ and $10.1 \%$ of nonHispanic Black Caribbeans attained at least a high school education and were unemployed compared to Blacks in the Northwest $(74.6 \% ; 19.7 \%)$ and Southwest $(64.5 \% ; 19.2 \%)$ of the US, respectively. In the Singh and Siahpush (2002) report, $18 \%$ and $1.4 \%$ of non-Hispanic Blacks had more than 16 years of education and had a family income in excess of $\$ 50,000$ compared to their US-born counterparts $(8 \%, 0.9 \%)$, respectively. The authors did not report if these differences in the socio-economic parameters they observed were significant. The report by Logan and Deane (2003) showed Afro Caribbeans were better educated, have higher median household incomes, 
and typically live in better neighborhoods with higher socio-economic standings compared to African Americans. This maybe due to selective migration (Logan and Deane, 2003) by the Afro Caribbeans based on their educational attainment or their plans for higher education.

Although limited data are available about the pathways through which SES influences health-related outcomes, one hypothesis is that SES may work through chronic stress (Baum et al., 1999). This report stated that chronic stress burden is normally associated with overcrowding, crime, noise pollution, discrimination, personal distress, mental health problems and health-impairing behaviors.

In our study, a negative association between SES and PS in males compared to females was observed. Additionally, the relationship between SES, total cholesterol (TC) and low-density lipoprotein cholesterol (LDL-C) were inconsistent. Higher education was positively associated with TC and LDL-C in USBCA and LDL-C and TC/HDL-C ratio in AA participants, whereas higher household income was negatively associated with TC and LDL-C in FBCA participants, which is in the direction associating lower SES with CHD. Other investigators have reported an association between SES and CHD (AHA Update, 2002; Kaplan and Keil, 1993; Wood et al., 1998; Davey et al., 1996). It is suggested that individuals with lower SES may have higher cumulative levels of stress and that biological reaction to this stress may contribute to hypertension (Kington and Smith, 1997; Williams, 1992), overeating and/or increased cigarette smoking (AHA Statistical Update, 2002). 


\section{Knowledge of CHD Risk Factors}

Little is known about the attitudes and knowledge of CHD risk factors in individuals and the factors will motivate individuals to effect changes in their behaviors in an attempt to reduce the onset of chronic diseases, such as CHD are not properly understood (shepherd, 1998; Erhardt and Hobbs, 2002). In our study, knowledge of CHD risk factors was relatively high (mean $18.1 \pm 1.9$; range $0-25$, where 0 is low knowledge and 25 is very good knowledge) and, higher in females compared to males. However, there were no differences between the ethnic groups with respect to knowledge of CHD risk factors. Interestingly, good knowledge of CHD risk factors did not translate into action as FBCA participants were less likely to be physically active, whereas AA participants with high knowledge of CHD risk factors were more likely to have high BMI (an indication of sedentary lifestyle). This is in agreement with the report of Shepherd (1998) that showed individuals in 5 European countries (France, Germany, Italy, Sweden, United Kingdom) to have considerable degree of indifference to $\mathrm{CHD}$, despite the possession of a reasonable level of knowledge of CHD risk factors and, even among individuals with $\mathrm{CHD}$.

\section{Family History of CHD}

There is evidence to indicate that a family history of premature CHD (definite myocardial infarction or sudden death before 55 years of age in father or other male firstdegree relative, or before 65 years of age in mother or other female first-degree relative) is an independent risk factor for coronary events even when other risk factors are absent (Benson et al., 1999; Li et al., 2000; Williams et al., 2001). Coronary heart disease tends 
to cluster in families and a positive family history of premature CHD counts as a risk factor (NCEP: ATP III, 2001). Children of parents with heart disease are more likely to develop the condition themselves, probably due to shared socio-cultural environment, exposures to similar behavioral habits and genetics (NCEP: ATP III, 2001). In our study, the reported presence of a family history of CHD was higher in FBCA (39.4\%) participants compared to AA (31.1\%) and USBCA (25.8\%) participants, although the differences were not significant. Maybe the variation in this parameter could be due to the information about family health issues not being discussed or shared with siblings. In our study we observed that the percentage of the USBCA (51.6\%) participants who reported that they did not know their family history of CHD was higher compared to the FBCA (30.3\%) and AA (32.8\%) participants. However, a positive family history of CHD captures the underlying complexities of gene-gene and gene-environment interactions by identifying families with combinations of risk factor, both measured and unmeasured, that lead to disease expression (Hunt et al., 2003). Our results showed males who had a positive family history of CHD were smokers an indication that this knowledge did not influence their smoking habits. Also, USBCA participants with a positive family history of CHD were more likely to have elevated TC, LDL-C and hs-CRP. Thus, targeted screening and intensive intervention (Hunt et al., 2003) in this population may prevent the early onset of cardiovascular evens in the future. There is a very limited data on the relationship between family history of CHD and different ethnic groups or the effects on behavioral/environmental parameters. 


\section{Health Insurance and Visitations to Healthcare Professionals}

Health Insurance is the key that provides access to high quality health care and consequently to better health", so states a recent Institute of Medicine (IOM) report (IOM, 2002). Nearly 30 million working adults are uninsured. The uninsured receive too little medical care and receive it too late, are generally sicker and die earlier and receive poorer care during visitations to the hospital or doctor (IOM, 2002). Our study results showed that more FBCA (71.2\%) participants had medical insurance coverage compared to USBCA (69.4\%) and AA (67.2\%) participants, however, the USBCA participants (85.5\%) were more likely to visit a Healthcare Professional (HCP) during the past year compared to FBCA $(80.3 \%)$ and AA $(73.8 \%)$ participants. Since most of the FBCA participants were international students, then registration under the university's health plan was compulsory. The differences in visitations to the HCP may mirror differences in beliefs, attitude and values of how each group perceives illness and disease or possibly mistrust of the health care system, which lead to less willingness to use the health care system (LaVeist et al., 2000a,b). Our result is in contrast to the report of Singh and Siahpush (2002) which indicated that the percentage of US-born non-Hispanic Blacks $(40.2 \%)$ who had insurance coverage was higher compared to foreign-born non-Hispanic Blacks (34.5\%). 


\section{Research Question 2: Are FBCA adults less likely to have behavioral risk factors for CHD as compared to USBCA and AA adults?}

\section{Dietary Intakes}

Krauss et al. (2000) have indicated that populations consuming diets high in fruits, vegetables, whole grains and unsaturated fatty acids may be at lower risk for many of the chronic degenerative conditions, such as $\mathrm{CHD}$, obesity, hypertension and diabetes. There is evidence from animal experiments, observational studies, clinical trials and metabolic studies conducted in different populations showing strong and consistent relationship between dietary fats and CVD, especially CHD (Kris-Etherton et al., 2001). The American Heart Association recommends a diet low in saturated fat, trans fat, cholesterol and sodium. It should also be high in vegetables, fruits, whole grains, legumes, fat-free or low-fat dairy products, and dietary fiber (AHA Statistical Update, 2002). The total fat (saturated, trans, monounsaturated, polyunsaturated) intake should be less than $30 \%$ of total daily calories. Saturated fat and trans fat should not exceed $10 \%$ of total daily calories for healthy individuals.

In general, the findings of our study have showed that the foreign-born Afro Caribbean (FBCA) individuals had a better diet profile compared to their US-born counterparts. The dietary findings included the observations that the FBCA participants compared to the USBCA and AA participants consumed significantly less total fat, saturated fat, percent energy from fat, fat servings, dietary cholesterol and sodium and more percent energy from carbohydrate and vegetables. A similar trend was seen for the FBCA males compared with the USBCA and AA males. For the FBCA females, only the 
mean dietary cholesterol intake was significantly lower compared to the USBCA and AA females.

Reports from the BRFSS 2000 (CDC/NCHS, 2000) survey showed that $81 \%$ of males and $73 \%$ of females consumed fewer than 5 servings of fruits and vegetables each day. This report also states that more than $60 \%$ of young adults eat too much fat (fat provided more than $30 \%$ of total energy intake) whereas less than $20 \%$ ate the recommended 5 or more servings of fruits and vegetables each day. Our study showed similar trends as $70 \%$ of the males and $53 \%$ of the females consumed too much energy from fat (fat provided more than $30 \%$ of total energy intake) of whereas less than $20 \%$ and $30 \%$ of males and females, respectively, consumed less than 2 servings of fruits and vegetables daily. Also, the percentages of participants exceeding dietary recommendations for percent energy from fat, saturated fat, cholesterol and sodium and not meeting these recommendations for fruit consumption were higher for USBCA and AA participants compared to the FBCA participants. Thus, based on these dietary findings, the USBCA and AA participants may be at greater risk of CHD events in the future, since evidence from animal experiments, observational studies, clinical trials and metabolic studies conducted in different populations have showed strong and consistent relationships between dietary fats and CVD, especially CHD (Kris-Etherton et al., 2001). In our study, high diet score (better diet) correlated positively with high-density lipoprotein cholesterol (HDL-C) in AA participants, indicating the close relationship between diet and dyslipidemia. Our study is in agreement with other studies showing a relationship between diet, dyslipidemia and CHD (Kruass et al., 2000; Willett, 1998). Also, low vegetable and fiber consumption in USBCA participants was associated with 
high TC, whereas high dietary cholesterol consumption by the AA participants was associated with low HDL-C levels. These findings are in agreement with the Framingham Heart Study (Wilson et al., 1998), the Multiple Risk Factor Intervention Trial (MRFIT) (Stamler et al., 1986), and the Lipid Research Clinics (LRC) trial (Lipid Research Clinics Program 1984a,b) which showed a direct relationship between LDL-C (or TC) and the rate of new onset of CHD in men and women who were initially free of CHD. Also, there is strong epidemiological evidence indicating that low levels of HDL-C increase CHD mortality and morbidity (Wilson et al., 1998; Gordon et al., 1989; NCEP: ATP III, 2001). In our study, the fat, dietary cholesterol, vegetable and sodium components of the diet were the major differences between the ethnic groups. In England, Sharma et al (1999) have shown that foreign-born Afro Caribbeans eat less fat compared to the United Kingdom-born (UK-born) Afro Caribbeans and the general UK population. This may be due to the influence of the social environment on the food selection process (Anderson, 2003) a concept not investigated in this study and, an important area of research in the future. Understanding the social context of food choice may be as important as identifying the food sources of the major nutrients in food (Anderson, 2003). This is because food provides a means to demonstrate cultural identity and is also symbolic of many areas of the participants' origin, religious background and beliefs structures (Anderson, 2003). Bonow and Eckel (2003), have suggested support for a "heart-healthy" balanced diet. They suggested that this diet should consist of a wide variety of fruits and vegetables, whole grains, legumes, lean meat, poultry and fish, with the percentage intakes of total fat accounting for less than $30 \%$ and, saturated and trans fatty acids less than $10 \%$ of the total calories, respectively. Advise from the American Heart Association 
(AHA statistical Update, 2004) is in agreement with this suggestion. The AHA recommends a diet low in saturated fat, trans fat, cholesterol and sodium. It should also be high in vegetables, fruits, whole grains, legumes, fat-free or low-fat dairy products, and dietary fiber. The total fat (saturated, trans, monounsaturated, polyunsaturated) intake should be less than $30 \%$ of total daily calories. Saturated fat and trans fat should not exceed $10 \%$ of total daily calories for healthy individuals.

Poor nutrition and physical inactivity together are the second leading preventable cause of death in the US. Poor nutrition and physical inactivity are responsible for an estimated 300,000 deaths each year and are causally related to a variety of chronic diseases and conditions, including overweight and obesity, high blood pressure, hyperglycemia and diabetes, hypercholesterolemia, CHD, stroke, osteoarthritis and some cancers (FLDH, 2001). A healthy diet is important for preventing heart disease and stroke (Wood et al., 1998; AHA Statistical Update, 2002).

\section{Physical Activity/Inactivity}

The mean physical activity index (PAI), work activity index (WAI), sports activity index (SAI) and leisure-time time activity index (LTAI) scores of the participants were generally low and did not differ significantly between the ethnic groups or the gender-ethnic male and female subgroups. More USBCA and AA participants did not play a sport (indication of sporting activities) and watched TV often/very often (indication of leisure-time physical inactivity) compared to FBCA although the differences did not reach statistical significance. There is evidence indicating that physical inactivity is a major risk factor for CHD (National Cholesterol Education 
Program [NCEP]: Adult Treatment Panel [ATP] III, 2001; Fletcher et al., 1996; US Surgeon General Report, 1996). The relative risk of CHD associated with physical inactivity ranges from 1.5 to 2.4 , an increase in risk comparable to that observed for high blood pressure, high blood cholesterol or cigarette smoking (AHA Statistical Update, 2002; Pate et al., 1995). Individuals who are less active have a 30-50\% greater risk of developing high blood pressure compared to those who are more active (AHA Statistical Update, 2002; Haskell et al., 1992).

In our study, the mean PAI was moderate $(7.8 \pm 1.4$; range $3.0-15.0$, where 3.0 is low PAI and 15.0 is high PAI scores) and did not differ significantly between the ethnic groups. The USBCA participants who were physically inactive were more likely to have elevated TC and LDL-C and total cholesterol/high-density lipoproten cholesterol (TC/HDL-C) ratio, observations that were in the direction showing an association between physical inactivity and CHD (NCEP: ATP III, 2001; Fletcher et al., 1994; US Surgeon General Report, 1996). Also, the USBCA participants who were physically inactive were more likely to have elevated hs-CRP levels suggesting that physical activity may reduce hs-CRP levels. Indeed there is evidence to this effect (Smith et al., 1999; Tisi et al., 1997). Watching television often/very often was positively associated with DBP in FBCA participants and elevated TC/HDL-C in AA participants, demonstrating the importance of increased physical activity on risk factors for CHD in this and other population. 
Evidence from the Framingham Heart Study has indicated that obesity is strongly predictive of CHD and, that risk for cardiovascular disease is increased when abdominal obesity is present. In our study, overweight was defined as body mass index (BMI) 25.0$29.9 \mathrm{kgm}^{-2}$ and obesity BMI $\geq 30.0 \mathrm{kgm}^{-2}$. Central obesity was defined as waist circumference (WC) $\geq 35$ inches ( 88 centimeters) in women and $\geq 40$ inches (102 centimeters) in men (World Health Organization [WHO], 1997; National Heart, Lung and Blood Institute [NHLBI], 1998; NCEP: ATP III, 2001). The mean BMI (24.8 \pm 4.0$)$ and WC $(32.6 \pm 4.1)$ score of FBCA participants were lower compared to the USBCA $(26.2 \pm 4.8,33.5 \pm 4.6)$ and $\mathrm{AA}(26.7 \pm 6.6,33.5 \pm 5.2)$ participants. Also, the percentages of FBCA participants who were obese (7.6\%) and had increased abdominal girth $(10.6 \%)$ were lower compared to USBCA $(17.7 \%, 16.1 \%)$ and AA $(23.0 \%, 16.4 \%)$ participants. Similar findings have been documented by the studies of Singh and Siahpush (2002). Using data from the National Longitudinal Mortality Study (1979 through 1989), they showed that the mean BMI score of foreign-born non-Hispanic Black (25.6) adults was lower compared to the US-born non-Hispanic Black (27.1) adults. This same study showed that foreign-born non-Hispanic Blacks (25.2\%) were less likely to be overweight compared to their US-born (38.4\%) counterparts. Our study has also been corroborated by a report from the National Academy of Sciences (Harris, 1999) reporting that foreign-born young adults were less likely than their US-born young adult counterparts to be obese ( $17.0 \%$ versus $26.7 \%)$. 
We observed positive associations between BMI/WC and, systolic/diastolic blood pressures (S/DBP), TC, LDL-C in USBCA, S/DBP in AA participants and DBP in FBCA participants. Also, BMI/WC was negatively associated with HDL-C in FBCA, USBCA and AA participants. These findings are consistent and in the direction of reports showing relationships between BMI and these parameters that are associated with CHD (NCEP: ATP III, 2001; AHA Statistical Update, 2004; Koenig et al., 1999; Rhode et al., 1999). Our study is also in agreement with the report of Folsom et al (1989) which demonstrated that central or abdominal obesity was negatively correlated with HDL-C in both Blacks and Whites. The positive association between BMU/WC and TC, LDL-C in the USBCA participants compared to the FBCA participants could be due to the higher percentages of the USBCA participants who were overweight and obese or who had poorer diets compared to the FBCA participants. It is suggested that although body fat is metabolically active it cannot by itself cause CHD but may be an index of underlying metabolic disturbances and that could directly impinge on atherogenesis and CHD (Zoratti, 1998). Body fat also plays a leading role in increasing the flux of free fatty acids (FFA) carried to the liver from abdominal adipocytes (Marin et al., 1992) with the possibility of the excess FFA interacting with the secretions of lipoproteins (McKeigue et al., 1991) and possibly influencing TC and LDL-C levels.

A positive association between BMI and hs-CRP was also observed in males, females and all three ethnic groups. Our results are similar to that of Saito et al (2003) who suggested that body fat may directly influence hs-CRP levels, since adipocytes are known to secrete interleukin-6, the primary hepatic stimulant of hs-CRP (Visser et al., 1999; Yudkin et al., 1999) and, may of also synthesize and secrete hs-CRP (Pepys And 
Hirschfield, 2003). Our study is also similar to the National Health and Nutrition Examination Survey (NHANES II) study (Wilson et al., 1998) that showed a positive association between BMI and hs-CRP levels, with the occurrence of elevated hs-CRP found to be twice as likely in obese men and six times more likely in obese females. Other studies (Yudkin et al., 1999; Festa et al., 2000; Heald et al., 2003) have also found similar associations between hs-CRP and BMI. Also, BMI was positively associated with TC/HDL-C in the males and all three ethnic groups but did not achieve significance for the AA participants. Scranton et al (2004) have also demonstrated a positive association between increased weight or failure to exercise and TC/HDL-C ratio. They demonstrated that physicians who gained weight $\left(B M I \geq 25.0 \mathrm{~kg} / \mathrm{m}^{2}\right.$ ) were twice as likely to have a TC/HDL-C ratio $\geq 5.0$ compared with physicians who maintained normal weight $(\mathrm{BMI}<$ $25.0 \mathrm{~kg} / \mathrm{m}^{2}$ ). Also, physicians who stopped exercising were $40 \%$ more likely to have TC/HDL-C ratio $\geq 5.0$.

Additionally, the positive association between BMI and cigarette smoking in USBCA and BMI and PAI in AA participants compared to FBCA participants may suggest that USBCA and AA participants may gain weight by other measures, such as the type and quality of their diets and possibly, time spent watching television (TV). We observed significant differences between the ethnic groups with respect to dietary habits. Although there was no significant difference in the amount of time spent watching TV between the ethnic groups, the percentages of USBCA and AA participants who watched TV was higher compared to FBCA participants. Positive associations between obesity and time spent watching TV, have also been reported by other investigators (Dietz and Gortmaker, 1985; Gortmaker et al., 1990; Tucker and Bagwell, 1991; Buchowski and 
Sun, 1996). Although we did not detect any consistent association between BMI and the physical activity index, this could indicate that we may not have adequately identified all the activities that contribute to energy expenditure in this population. The physical activity questionnaire used in this study did not include any questions about housework (Pomerleau et al., 1999), an import source of energy expenditure for students especially women in this population. Thus, more research is needed in assessing physical activity in this population. These findings are in agreement with reports showing that obesity especially central obesity is a risk factor for many chronic diseases including cardiovascular disease (CVD) (Willett, et al., 1995) and possibly death (Folsom et al,, 1993). Obesity is of public health importance affecting not only the individuals in the US population but also individuals in the developing world (Eckel, 1997). Knowledge of, and awareness of the complications of obesity may help motivate individuals in their weight management efforts (Williamson et al., 2000). This is important since Afro Caribbean and African American women with fuller figures are more acceptable to their male counterparts whereas, non-Hispanic White females with fuller figures maybe despised by their male counterparts (Anderson et al., 2002). Also, in a study of elderly women in the US, African Americans were more likely than white women to report that they were satisfied with their weight and less likely to diet (Stevens et al., 1994).

\section{Cigarette Smoking}

There is evidence to support the claim that cigarette smoking is a contributor to the risk for CHD and other forms of CVD (Kannel et al., 1980; Colditz et al., 1988; LaCroix, 1991; McBride, 1992; Jonas et al., 1992; Pyörälä et al., 1994). This relationship 
is dose related and smoke cessation will reduce the risk for CVD events within months of quitting (US Surgeon General Report, 1990).

In our study, more USBCA (4.8\%) and AA (6.6\%) individuals smoked cigarettes compared to the FBCA $(0.0 \%)$ individuals. A similar trend for cigarette smoking was seen in the report of Singh and Siahpush (2002). They reported that the percentage of foreign-born Blacks (10.4\%) who was current smokers was lower compared to US-born Blacks (29.3\%), whereas in our report FBCA participants were all nonsmokers. The AA participants were generally older than the FBCA and USBCA participants and, a positive association was observed between age and cigarette smoking in the AA participants. In the US, as many as $30 \%$ of all deaths due to CHD are attributable to cigarette smoking or, being exposed to environmental tobacco smoke at home or work, with the risk being dose-related (AHA Statistical Update, 2004). Cigarette smoking also acts synergistically with other risk factors, thus substantially increasing the risk of CHD (Ockene and Miller, 1997). Our study demonstrated a positive association between cigarette smoking and hsCRP levels in USBCA participants. Our results agree with other reports showing that cigarette smoking, which seems to promote arterial inflammation and predispose to major coronary events, was associated with higher hs-CRP levels (Tracy et al., 1997b) and, possibly risk of CHD.

\section{Alcohol Consumption}

Although there is a complex relationship between alcohol consumption and CHD, with moderate consumption of alcohol showing reduction in CHD, risky drinking has been associated with health risks (Pearson, 1996). Compared to the USBCA (29.0\%) and 
FBCA (24.2\%) participants, AA (50.8\%) participants consumed more alcoholic beverages each month. Our findings are in agreement with those of Hicks et al (2003). Using data from NHANES III, they showed that the percentages of African Americans from the North (Northeast, Midwest or West census regions) (56.8\%) or South census regions (47.5\%) who consumed more than $12 \mathrm{~g}$ alcoholic beverages per day to be higher than that of Black immigrants (39.7\%). Of interest $72 \%$ of individuals less than 21 years old were consuming alcoholic beverages with $34 \%$ consuming in excess of one drink per month. This could be due to the easy availability of alcoholic beverages on the university campus or, younger individuals may consume alcoholic beverages purchased by older persons.

In our study, we observed a positive association between alcohol consumption and triglyceride (TG) levels in males compared to females indicating an association between risky drinking with health risk (Pearson, 1996). There is epidemiological evidence indicating a positive relationship between serum triglyceride (TG) levels and incidence of CHD (Austin et al., 1998; Assmann et al., 1998a; NCEP: ATP III, 2001). In our study, we also observed a negative association between alcohol consumption and systolic blood pressure (SBP) levels in USBCA participants but not FBCA and AA participants. Moderate alcohol consumption has been shown to be associated with reduction in CHD risk (Pearson, 1996). 


\section{Research Question 3: Are FBCA adults less likely to have bio-physiological risk factors for CHD as compared to USBCA and AA adults?}

\section{Blood Pressure}

A recent report $\left(7^{\text {th }}\right.$ Report of the Joint National Committee on Prevention, Detection, Evaluation and Treatment of High Blood Pressure [JNC] VII, 2003) has classified normal $\mathrm{BP}$ as systolic $\mathrm{BP}<120 \mathrm{~mm} \mathrm{Hg}$ and diastolic $\mathrm{BP}<80 \mathrm{~mm}$. Hypertension is the most potent risk factor for stroke. It is also a major risk factor for CHD, kidney failure and heart failure (JNC VI, 1997). The percentages of FBCA (25.8\%; $29.0 \%$ ) participants with elevated SBP and DBP were higher compared to the USBCA $(13.3 \% ; 26.7 \%)$ but lower than values for the AA $(26.7 \% ; 50.0 \%)$ participants, respectively, although these differences were not significant. In the report by Hicks et al (2003), hypertension was defined as S/BBP $\geq 140 / 90 \mathrm{~mm} \mathrm{Hg}$ and they reported the percentage of Black immigrants with hypertension (48\%) to be lower than African Americans in the northern (72\%) and Southern (80\%) census regions, respectively, whereas Singh and Siahpush (2002) reported a much lower prevalence of hypertension in foreign-born non-Hispanic Blacks (17\%) compared to US-born non-Hispanic Blacks $(25 \%)$, using self-reported data on hypertension. However, like our study these differences were not statistically significant. However, significantly lower rates of hypertension were reported for Caribbean and African Blacks compared to US Blacks in the International Collaborative Study of Hypertension in Blacks (ICSHIB) (Cooper et al., 1997a). Possible reasons for these different results may be due to different methodologies, or the fact that majority of our participants were young students. The 
FBCA participants are in the process of adjusting to a new environment and higher blood pressure values may reflect this acclimatization process (Singh and Siahpush, 2002). In our study, BMI was found to be associated positively with S/DBP in males and females, DBP in FBCA participants and, S/DBP in USBCA and AA participants indicating that obesity is a powerful determinant for the development of hypertension and CHD (JNC VII, 2003). In our study, a positive association was observed between S/DBP and hs-CRP in females and between DBP and TC/HDL-C ratio in males. This is in agreement with other studies (Koenig et al., 1999; Rhode et al., 1999) showing positive associations between S/DBP and CVD risk factors, including hs-CRP. In the Koenig et al (1999) study, they investigated and followed, for 8.2 years, 936 men between the ages of 45 to 64 years, who participated in the first Monitoring Trends and Determinants in Cardiovascular Disease (MONICA) Augsburg study, in Germany, between 1984 and 1985. They demonstrated that hs-CRP levels varied significantly with age, BMI, TC, smoking status, blood pressure and diabetes and, slightly with alcohol consumption, education, leisure-time physical activity and work activity. In the Rohde et al (1999), they investigated 1172 apparently healthy middle-aged (40-84 years) men who participated in the Physician Health Study. They showed significant positive associations (all $\mathrm{p}$ values $<$ 0.05 ) between hs-CRP levels and age, number of cigarettes smoked each day, BMI, S/DBP, TC, triglyceride (TG), lipoprotein (a) [Lp (a)], apolipoprotein B (apo B), tissuetype plasminogen activator antigen, total homocysteine and fibrinogen. 


\section{Blood Glucose}

Impaired fasting blood glucose levels $(110-125 \mathrm{mg} / \mathrm{dL})$ is an indicator of insulin resistance and is generally accompanied by other metabolic risk factors (Tripathy et al., 2000; NCEP: ATP III, 2001). Some individuals with impaired fasting blood glucose will eventually develop type 2 diabetes (Lindahl et al., 1999), which further enhances the risk for CHD. Type 2 diabetes is the epitome of the metabolic syndrome, characterized by abdominal obesity, atherogenic dyslipidemia (elevated triglyceride, small low-density lipoprotein cholesterol particles, low high-density lipoprotein cholesterol), elevated blood pressure, insulin resistance, pro-thrombotic and pro-inflammatory conditions (NCEP: ATP III, 2001). This disorder is associated with increased risk of CHD and occurs in $24 \%$ of the US adult population (Ford et al., 2002). Over 600,000 individuals are diagnosed with DM each year. Diabetes accounts for over 200,000 deaths and $\$ 18.9$ million in ambulatory visits annually (Center of Disease Control (CDC) Diabetes Factsheets, 2000).

In this study, the mean fasting blood glucose (FBG) levels of the three ethnic groups were normal $(<110 \mathrm{mg} / \mathrm{dL})$ and did not differ significantly. However, impaired fasting blood glucose (110-125 mg/dL) levels were detected in $6.1 \%, 6.5 \%$ and $6.6 \%$ of FBCA, USBCA and AA participant, respectively. These individuals were indeed unaware of their condition and may indicate a subset of this young adult population at risk for diabetes mellitus and CHD. There is evidence indicating that over 16 million (12 million diagnosed) Americans, nearly $6 \%$ of the US population, have diabetes mellitus, with $50 \%$ (5.9 million) of these individuals unaware of their condition (CDC Diabetes Factsheets, 2000). 
Symptoms associated with the metabolic syndrome (MS) were observed in $4.2 \%$ of this young adult population. This is much lower than that reported by Ford et al (2002). A low percentage of participants in the present study met the criteria for the MS: $1.5 \%, 1.6 \%$ and $3.2 \%$ of FBCA, USBCA and AA participants, respectively. This indicates that a small subset of individuals are showing early signs of CHD risk and, may indeed develop CHD in the future if behavioral and other risk factors are not addressed. This is the first report that addressed the differentials in the metabolic syndrome of foreign-born and US-born individuals.

In our study, higher perceived stress (PS) was associated with fasting blood glucose (FBG) levels in AA participants, whereas cigarette smoking was positively associated with FBG levels in females, indicating the harmful effects of PS and cigarette smoking on health outcomes. There is evidence showing that the effect of stress on the hypothalamic-pituitary-adrenal (HPA) axis, which plays a central role in the homeostatic process, is the increased production of glucocorticoids (cortisol, catecholamines), which have been shown to have diabetogenic effects (Dalman et al., 1993; Rosmond, 2005) and, in fact contribute to the entire spectrum of the metabolic syndrome, including central obesity, hyperglycemia, dyslipidemia and other risk factors for CHD (Björntorp, 1993; Rosmond, 2004).

\section{Total Cholesterol and Low-density Lipoprotein Cholesterol}

There is strong epidemiological evidence demonstrating that CHD is positively correlated with TC and LDL-C (Gordon et al., 1989, Wilson et al., 1998, NCEP: ATP III, 2001; AHA Statistical Update, 2004). In our study, the percentages of males with TC 
levels above $200 \mathrm{mg} / \mathrm{dL}$ and LDL-C above $130 \mathrm{mg} / \mathrm{dL}$ were $27.5 \%$ and $30.8 \%$, respectively, whereas in the females, the situation was reversed with $\mathrm{TC}$ levels above 200 $\mathrm{mg} / \mathrm{dL}$ and LDL-C above $130 \mathrm{mg} / \mathrm{dL}$ being $34.7 \%$ and $27.6 \%$, respectively. The American Heart Association has reported similar results (AHA Statistical Update, 2004). The report showed the percentages of male African Americans with TC levels above 200 $\mathrm{mg} / \mathrm{dL}$ and LDL-C above $130 \mathrm{mg} / \mathrm{dL}$ were $37 \%$ and $46 \%$, respectively, whereas for the females, the values were $46 \%$ and $42 \%$, respectively.

The percentage of FBCA (25.8\%) participants with elevated TC was lower than the USBCA (37.1\%) and AA (31.1\%) participants, whereas the percentage of FBCA (27.3\%) with elevated LDL-C lower than USBCA (35.5\%) but not the AA (24.6\%) participants. This is the first report that addressed the differentials in the lipid profile of foreign-born and US-born individuals. In our study, we observed a strong and positive relationship between TC, LDL-C levels and the TC/HDL-C ratio in the male and female groups and the three ethnic groups, indicating the strong relationship between dyslipidemia and risk for CHD (Wilson et al., 1998, NCEP: ATP III, 2001; AHA Statistical Update, 2004). Scranton et al (2004) have also demonstrated an association between dyslipidemia and the TC/HDL-C ratio. There is evidence indicating that the total cholesterol/HDL-Cholesterol ratio (TC/HDL-C) is a powerful predictor for CHD risk (Kinosian et al., 1995; Criqui and Golomb, 1998).

\section{Triglyceride and High-density Lipoprotein Cholesterol}

There is strong epidemiological evidence indicating that low levels of HDL-C increase CHD mortality and morbidity (Wilson et al., 1998; Gordon et al., 1989; NCEP: 
ATP III, 2001). Many case-controlled and prospective studies have shown that HDL-C, TG or both are better predictors of individuals with or without CHD than TC or LDL-C (Nikkila et al., 1990; Simons et al., 1991; Stampfer et al., 1991; Romm et al., 1991; Genest et al., 1991; Buring et al., 1992).

In our study, the TG levels were similar between the gender and ethnic groups. However, the percentage of males $(35.2 \%)$ with low HDL-C $(<40 \mathrm{mg} / \mathrm{dL})$ was significantly higher compared to the females (8.2), whereas, there were no significant differences between the percentages of the ethnic groups with low HDL-C levels. The AHA have reported similar trends (AHA Statistical Update, 2004) They reported that low HDL-C levels were present in $24 \%$ of African American males and 13\% of females. There is evidence indicating that a $1 \%$ decrease in HDL-C is associated with a $2-3 \%$ increase in CHD risk (Gordon et al., 1989) whereas high HDL-C levels have been associated with longevity and are indeed protective against CHD (NCEP: ATP II, 2001; Toth, 2004). For example, in the Framingham study (Castelli et al., 1986) low HDL-C levels (below $40 \mathrm{mg} / \mathrm{dL}$ ) have been shown to increase sharply the risk of CHD and, this relationship was independent of TG levels as demonstrated by The Prospective Cardiovascular Munster Study (Assman and Schulte, 1992).

In our study, we observed a strong and positive relationship between TG levels and hs-CRP in males. It is suggested that a possible pathway through which dyslipidemia influence CHD maybe through inflammation, now considered a risk factor for CHD (ATP III, 2001; Ross, 1999). Also, there were strong and positive associations between TG and TC/HDL-C ratio in males and USBCA participants and, strong and negative associations between HDL-C and the TC/HDL-C ratio in males and females and the three 
ethnic groups. It is suggested that a possible mechanism for the cardio-protective effects of HDL-C could be through a reverse cholesterol transport pathway, or inhibition of lipid peroxidation (Mackness and Durrington, 1995), cellular adhesion (Barter, 1997) and/or platelet activation (Sugatani et al., 1996).

Research Question 4: Are FBCA adults less likely to have emerging risk factors for CHD as compared to USBCA and AA adults?

\section{C-reactive Protein}

Lipid deposition in the vascular system has been thought to be the major cause of $\mathrm{CHD}$, and thus aggressive cholesterol testing has been used as a tool to identify individuals at increased risk for CHD. Yet $50 \%$ of all myocardial infarctions and strokes occur in individuals with normal or slightly elevated TC (Kannel, 1995) but elevated hsCRP levels (Rifai and Ridker, 2001; Ridker, 2004). There are reports showing an association between hs-CRP and low-grade inflammation, an important determinant of atherothrombosis, a leading cause of morbidity and mortality in the world (NCEP: ATP III, 2001; Libby et al., 2002; Zeltser et al., 2004). Measurement of hs-CRP is now considered a sensitive marker of chronic low-grade vascular inflammation, which has been implicated in the pathogenesis of atherosclerosis (Koenig et al., 1999). There is evidence indicating that serum hs-CRP levels are increased many years in advance of the first coronary and cerebrovascular events in healthy (Ridker et al., 1997; Ridker et al., 1998b; Koenig et al., 1999) and high-risk individuals (Tracy et al., 1997b; Kuller et al., 1996). In two of these studies (Ridker et al., 1998a, 2000), hs-CRP was shown to be a 
powerful indicator of future cardiovascular events even among subgroups of women with no history of hyperlipidemia, hypertension, smoking, diabetes or family history of CHD. There is additional evidence demonstrating that individuals with elevated hs-CRP were 4 times as likely to have a cardiovascular event as those with low hs-CRP. Additionally, in the Women' Health Study (Ridker et al., 2002), risk of CVD was markedly elevated in women with elevated hs-CRP and normal LDL-C $(<130 \mathrm{mg} / \mathrm{dL})$ indicating that hs-CRP provides risk prediction better than LDL-C alone, that it identifies high risk individuals who would not be considered to be at high risk on the based on LDL-C or other traditional risk factors, and that hs-CRP adds substantial predictive information to traditional risk evaluation (Ridker, 2004). Most of the information about hs-CRP in ethnic groups originated from Europe where hs-CRP levels of Afro Caribbeans were generally lower than UK-born individuals (Heald et al., 2003). However, studies in the US (Wener et al., 2000; Wong et al., 2001) have shown that hsCRP levels are normally higher in Blacks than Whites. However, there is no published data on hs-CRP differentials in foreign-born and US-born individuals.

In our study, the mean hs-CRP levels in FBCA were lower compared to USBCA and significantly lower compared to the AA participants. Also, the percentage of FBCA with elevated hs-CRP was lower than USBCA and significantly lower than the AA participants. Thus, USBCA and AA participants may be showing early signs of subclinical inflammation and, maybe at risk of early cardiovascular events compared to FBCA participants. Although no firm guidelines on treatment for elevated hs-CRP currently exists, there is evidence suggesting that hs-CRP adds predictive information to 
the other established CHD risk factors, such as TC and LDL-C (Ridker et al., 2002; Albert et al., 2003).

\section{Total Cholesterol/High-density Lipoprotein Cholesterol Ratio}

There is evidence indicating that the TC/HDL-C is a powerful predictor for CHD risk (Kinosian et al., 1995; Criqui and Golomb, 1998). A TC/HDL-C ratio of $\leq 3.5$ (males and females) is associated with a lower risk for CHD whereas TC/HDL-C ratios of $\geq 5.0$ are associated with higher risk for CHD (Sonnenberg et al., 1996; Kannel, 1983).

In our study, the mean TC/HDL-C ratio of the USBCA participants was higher but not significantly than values in the FBCA and AA participants. Also, more USBCA participants (19.4\%) had elevated TC/HDL-C $(\geq 5.0)$ ratio compared to the FBCA (13.6\%) and AA (13.1\%) participants. Thus, based on the TC/HDL-C ratio alone, the USBCA participants maybe at very high risk for early CHD events compared to FBCA and AA participants. This is because Konosian et al (1995) and Grover et al (1995), using data from the Framingham Heart Study and the Lipid Research Clinics Population Prevalence Study, showed that the TC/HDL-C ratio is a better predictor of CHD risk, even when compared with newer risk factors for CHD, such as $\mathrm{Lp}(\mathrm{a})$, homocysteine, fibrinogen and tissue plasminogen activator antigen. Gotto et al (2000), using data from the Air Force/Texas Coronary Atherosclerosis Prevention Study indicated that even in individuals with average cholesterol levels, baseline TC/HDL-C levels were significant predictors of subsequent major coronary event while TC and LDL-C levels were not. 


\section{Additional Findings}

\section{Behavioral/Environmental}

Individuals with low numbers of risk factors for $\mathrm{CHD}$ have low prevalence of CHD (Stamler et al., 1999). Low prevalence of CHD is related to healthy behavioral activities such as healthy diet, not smoking, consumption of moderate amount of alcohol and maintaining healthy body weight (Stampfer et al., 2000). These behavioral risks/risk factors impose a significant burden on our health care system and, are indeed the most important and modifiable causes of mortality and morbidity from CHD and stroke (Mokdad et al., 2004). This is demonstrated in the Nurses', Health Study (Stampfer et al., 2000) which reported that females who ate a healthy diet, did not smoke, had moderate alcohol consumption and exercise regularly had an $84 \%$ reduction in cardiovascular events. Behavioral risks tend to cluster in individuals and populations (Niggs et al., 2002, Orleans et al., 1999; Solomon and Kington, 2002) and disease risk and health care costs are greatest for individuals with multiple behavioral risks (Orleans, 2004).

In general, the findings of our study have showed that the foreign-born Afro Caribbean (FBCA) individuals had a better behavioral/environmental (healthy diet as measured by the diet score, lower percentages not watching television, proxy for leisuretime inactivity, obese, smoking and drinking alcoholic beverages) profile compared to their US-born counterparts. Multiple behavioral risks are indeed common (Ford et al., 2001; Berrigan et al., 2003; Coups et al., 2004; Pronk et al., 2004) even in our relatively young adult population.

Comparisons of our results with other reports on behavioral risk factors are difficult due to differences in methodology, variables and study population. In our study, 
the percentage of individuals $(14.5 \%)$ with none of the 5 behavioral/environmental risk factors (unhealthy diet, watching television often/very often, obese, cigarette smoking, excess alcohol consumptions) was higher than that reported by Pronk et al (2004) $(10.8 \%)$, whereas the percentage of individuals $(15.9 \%)$ with $\geq 3$ behavioral risk factors was lower than the percentage of adults $(26.9 \%)$ who did not met $\geq 3$ behavioral guidelines in the same report. Data from the Pronk et al (2004) study compared whether participants between the ages 18 to 64 years, from a large Midwestern health plan in the US, met the recommended guidelines for behavioral factors such as high diet quality, being physically active, having healthy weight, not smoking and no/or moderate alcohol consumption. Other studies (Coups et al., 2004; Fine et al., 2004; Ford et al., 2001; Berrigan et al., 2003) reporting on 4 behavioral risk factors have demonstrated the high prevalence of multiple behavioral risk factors for CHD in the US, and the challenge this may present for heath care professionals and policy planners. Coups et al (2004) investigated physical inactivity, overweight, cigarette smoking and risky drinking (consumption of $\geq 5$ alcoholic beverages in a single day), whereas Fine et al (2004) reported on overweight, physical inactivity, cigarette smoking and risky drinking both using data from the National Health Interview Survey (2001). Coups et al (2004) reported that $11.0 \%$ of the participants had none or $\geq 3$ behavioral risk factors, respectively whereas Fine et al (2004) reported that $10.0 \%$ of the participants had none of the behavioral risk factors but that $17.0 \%$ had $\geq 3$ behavioral risk factors. Ford et al (2001) investigated healthy lifestyle factors such as not smoking, adequate fruit and vegetable intakes, adequate physical activity and normal body weight in adults $\geq 21$ years whereas 
Berrigan et al (2003) investigated patterns of health behavior by characterizing adherence to public health recommendations for behavior related to exercise, tobacco, alcohol, dietary fat and fruit and vegetable intakes both using data from the NHANES III database for their studies. Ford et al (2001) showed that only $6.8 \%$ of the US population engaged in all 4 healthy lifestyle behaviors whereas Berrigan et al (2003) showed that adherence to recommendations concerning 5 health behaviors ranged from $34 \%$ to $74 \%$ (exercise, $40.6 \%$; tobacco use, $68.1 \%$; alcohol use, $73.9 \%$; percent energy from fat, $34.4 \%$; fruit and vegetable consumption, $35.1 \%$, respectively.

Most of the data on the clustering of behavioral activities are general in nature (Coups et al., 2004; Pronk et al., 2004; Berrigan et al., 2003; Ford et al., 2001; Fine et al., 2004) and have not reported on differences between ethnic subgroups. Also, most of these reports have included BMI, a proxy for sedentary lifestyle (Pronk et al., 2004; Coups et al., 2004; Ford et al., 2001; Fine et al., 2004), fruits, vegetables, dietary fat (Berrigan et al., 2003) or fruits and vegetables (Ford et al., 2001). Additionally, the percentage of males $(24.2 \%)$ with $\geq 3$ behavioral/environmental risk factors was significantly $(\mathrm{p}<0.01)$ higher than females $(8.2 \%)$. The percentage of the FBCA $(6.1 \%)$ participants with $\geq 3$ behavioral/environmental risk factors was significantly $(\mathrm{p}<0.05)$ lower than that of the USBCA (22.6\%) and AA participants (19.7\%). Similarly, the percentage of the FBCA ( $3.2 \%$ ) males with $\geq 3$ behavioral/environmental risk factors was significantly $(p<0.01)$ lower than the USBCA (36.7\%) and AA (33.3\%) males. Our study is in agreement with other studies (Coups et al., 2004; Fine et al., 2004; Pronk et al., 2004; Ford et al., 2001; Berrigan et al., 2003) demonstrating the high prevalence of multiple behavioral/environmental risk factors in the US, and indeed this population, and 
the challenge this may present for primary care and public health systems (Fine et al., 2004).

\section{Strengths of Study}

This is the first study of its kind presenting socio-demographic, behavioral/environmental, bio-physiological and emerging risk profiles for foreign and US-born Afro Caribbeans in South Florida. Although the Afro Caribbeans are considered part of the African American ethnic mosaic, they have different attitudes and behaviors towards health and healthcare and indeed are growing faster than other well-established ethnic groups, such as Cubans (Logan and Deane, 2003). There are over 1.5 million Afro Caribbean individuals living in the US with over 500,000 living in the tri-state counties of Miami-Dade, Broward and Palm Beach Counties (US Census, 2000). Individuals from the Caribbean Region are the fastest growing ancestry group in Broward, Palm Beach and Miami-Dade Counties in South Florida (US Census, 2000). For the year 1990 to 2000, the Caribbean population grew by $173 \%$ (from 55,197 to 150,476 ) in Broward County, $142 \%$ (from 20,441 to 49402 ) in Palm Beach County and $45 \%$ (from 105,477 to 153,255 ) in Miami-Date County (US Census, 2000). In spite of this increase in the foreign-born population of the US, there are relatively few studies investigating differentials in health, nutrition and mortality patterns between foreign-born and US-born individuals, especially Afro Caribbean Americans. The importance of this study is based on the realization that the US population is diversifying. Thus, distinctions based on national origins will become more visible and, therefore, healthcare policy planners and healthcare professionals must be aware of differences between the various ethnic groups. This will 
help in the formulation of culturally sensitive strategies and programs to improve health access and health outcomes in this and other populations. Also, with budget limitation for healthcare, properly identifying ethnic groups at increased risk for CHD can help in targeting this scarce resource more efficiently and effectively.

In the comparison of risk factors for CHD in this young and apparently healthy Black population, we demonstrated that some individuals had multiple risk factors for CHD. Indeed, differences in behavioral/environmental and emerging risk factors for CHD between FBCA, USBCA and AA participants were the major findings of this project. There were differences in socio-demographic and physiologic profiles between the ethnic groups, but these did not attain statistical significance, possibly due to small sample sizes. Data from the AHA Statistical Update, 2004 showed higher CVD events in males compared to females at younger ages. If these trends continue or if these risk factors for CHD remain unattended, then these can fuel increases in CVD events in the near future. The participants, in answering the self-administered questionnaires and receiving information on their health profile were more aware of their health status and this may provoke them to address health issues of concern to them. We also investigated the clustering of behavioral/environmental and metabolic risk/risk factors that provided more information about the health status of this population than would have been possible if the report was based on only a single factor (Pronk et al., 2004). The comprehensive behavioral risk measure and the established metabolic risk measure should be used more frequently, especially in the field of health promotion, disease prevention and health policy determinants. 


\section{Limitations of the Study}

The self-report nature of some aspects of the data collection process such as alcohol and cigarette use, in our young adult population, maybe reported with less accuracy (Dufour, 2001) and raises the possibility of recall bias. However, our results showed that a majority of the participants was knowledgeable about cardiovascular disease and, the test-retest results of our questionnaires showed high correlation. Thus, like Coups et al (2004) we were not aware of any evidence of systematic biases associated with recall of information in our study. To reduce information bias in this study, one investigator, using standardized and validated instruments collected and analyzed the data. To reduce self-reporting biases, assurances of confidentiality were given to the participants in order to encourage truthfulness. Accuracy of their responses on the questionnaires was encouraged as the participants were informed that a health profile would be given. Thus, for the health profile to be helpful, the accuracy of the information given is of vital importance. To minimize data extraction bias, all data was entered into SPSS, Version 12.0 by the Principal Investigator only and, the data checked for anomalies in order to ensure consistency in data extraction. Our research design was cross-sectional and, we may not be able to generalize our results beyond these ethniccultural groups from South Florida. The FBCA and USBCA populations comprise different groups such as Jamaicans, Haitians, Trinidadians, Barbadians and others from

the English-speaking Caribbean. These different groups of individuals may have different socio-economic backgrounds and behavioral habits. Thus, future research should investigate risk factors for CHD in the different Caribbean groups living in the Caribbean and compare these risk factors with US-born counterparts over time. 


\section{Summary}

Our study is corroborated by large studies such as the studies of Stephen et al (1994), Fang et al (1996; 1997) and Singh and Siahpush (2002). Stephen et al (1994), using data from the National Center for Health Statistics, have reported that foreign-born persons were generally healthier than the US-born population, although the health advantage varied by length of residence in the US. They also stated that in every measure of health status, and with regard to almost every socio-demographic variable, the most recent immigrants were healthier than foreign-born individuals who have lived in the US 10 years or more as well as healthier than the US-born population, although the differences were not as striking as between recent immigrants and the native-born population. There are some suggestions for this so-called "immigrant effect". It is suggested that (a) recent migrants may have been healthier than earlier migrants at the time of migration (Stephen et al., 1994), or physically fitter and more driven than those who remain in their country of origin (Singh and Siahpush, 2002), (b) earlier migrants may have been as healthy as recent migrant but that the health status of these earlier migrants may have deteriorated with increased duration of residence in the US (Stephen et al (1994) and, (c) these findings may reflect a combination of the above or other factors such as behavioral, psychological and environmental conditions known to influence health status, morbidity and mortality (Singh and Siahpush, 2002). This suggest that recent immigrants may have acquired behaviors that expose them to risk of chronic diseases in their new environment or that access to health care or visitation to health care professions may have been limited. In our study, although more FBCA (71.2\%) participants had medical insurance compared to USBCA (69.4\%) and AA (67.2\%) 
participants, USBCA (85.5\%) participants were more likely to visit with healthcare professionals during the past year compared to FBCA (80.3\%) and $\mathrm{AA}(73.8 \%)$ participants. A study from the National Academy of Sciences (National Academies of Sciences, Hernandez, 1999: access at http://www.nap.edu) has reported on the protective effect of immigrant status. This report demonstrated that foreign-born young adults experienced more favorable physical and emotional health and less involvement in risky behaviors than US-born young adults of foreign-born parents and US-born young adults of US-born parents. The report also stated that the protective quality of immigrant status was not related to the family or neighborhood context of immigrant children. Thus, it is suggested that future research should concentrate on uncovering the mechanism(s) behind the health protective effect(s) of foreign birth. Fang et al. (1997) showed 46\%-51\% lower mortality among black migrants compared to US-born black counterparts in New York City. In an earlier study, Fang et al. (1996) showed that Caribbean-born Blacks in New York City had substantially lower all-cause and cardiovascular mortality rates compared to Blacks born in the southern and northern region of the US. In this same study, Fang et al. (1996) have intimated that the risk of cardiovascular disease of foreign-born individual increases with length of residence in the US. Singh \& Siahpush (2002), using data from the National Longitudinal Mortality Study (1979 through 1989), a data set for the examination of socio-demographic and occupational factors associated with all-cause and cause-specific mortality in the US, found that immigrants tended to have more favorable health-enhancing behavioral habits than their US-born counterparts. They also showed that non-Hispanic black migrants compared to their US-born non-Hispanic black counterparts were less likely to smoke cigarettes (10.4\% vs. $29.3 \%)$, less likely to be 
overweight ( $25.2 \%$ vs. $38.4 \%)$, and less likely to be hypertensive $(16.8 \%$ vs. $25.5 \%)$ but more likely to have no medical insurance ( $40.2 \%$ vs. $34.5 \%)$. Our results are also in contrast to the report of Singh and Siahpush (2002) which indicated the percentage of US-born non-Hispanic Blacks (40.2\%) who had medical insurance coverage was higher compared to foreign-born non-Hispanic Blacks (34.5\%). The Fang et al (1996) and Singh and Siahpush (2002) reports did not inform if the differences they observed were significant. Also, Cairney and Ostbye (1999) showed that prevalence of excess body weights increased with time since migration to Canada. Prevalence of excess body weights increased from $33.5 \%$ and $17.1 \%(0-4$ years since immigration) to $52.6 \%$ and $42.1 \%$ (10 or more years since immigration) in males and females, respectively. Prevalence of excess body weights in male and female Canadians were $59.3 \%$ and $37.4 \%$, respectively. Additionally, in England, Sharma et al (1999) have also shown that as the length of residence of the foreign-born Afro Caribbean population increases, their eating habits approximates that of the eating habits of the native-born population.

There is a suggestion that ethnic groups within one location may adopt certain behaviors, whereas in another location this same ethnic group may behave differently (Yusuf et al., 2001) indicating a geographical transition of risks (Abbotts et al., 2004) for CHD. Differences in demographic profiles, environmental factors, early childhood programminig influences as well as differences in gene frequency or expression can influence variations in CVD between different populations (Yusuf et al., 2001) and indeed between different ethnic groups and ethnic subgroups within the same population. For example, in the Ni-Hon-San study of Japanese migrants, low TC and CHD rates were seen in Japanese men in Japan whereas intermediate and high TC levels and CHD rates 
were seen in Honolulu and San Francisco, respectively (Kagan et al., 1974). In England, comparison of Afro Caribbeans, South Asians and Europeans showed marked differences in central obesity, glucose intolerance hyperinsulinemia nd elevated dyslipidemia, despite having similar blood pressure, BMI and TC (McKeigue et al., 1991). Additionally, a study of individuals of West African Ancestry in Africa (Cameroon), the Caribbean (Jamaica) and England (Manchester) showed the prevalence of diabetes mellitus and hypertension was lowest in rural Cameroon and highest in Manchester (Cooper et al., 1997/1997a; Mbanga et al., 1999; Cruickshank et al., 2001). Thus, behavioral activities and culture rather than differences in genetics and clinical factors between foreign-born and US-born individuals may in part be explanatory for differences in CHD mortality between foreign- and US-born individuals as reported by Stephen et al (1994), Fang et al (1996) and Singh and Siahpush (2002).

\section{Conclusion and Recommendations}

Reports from several authors have demonstrated that foreign-born Black individuals have lower mortality and morbidity from CHD (Stephen et al., 1994; Fang et al., 1996; Singh and Siahpush, 2002; Hicks et al., 2003) and suggested that this maybe due to better health profiles compared to their US-born counterparts (Stephen et al., 1994; Singh and Siahpush, 2002; Hicks et al., 2003). In most of these reports (Fang et al., 1996; Stephen et al., 1994; Singh and Siahpush, 2002) reported differences between the foreign-born and their US-born counterparts were noted but whether these differences were statistically significant was not published. However, Hick et al (2003) reported statistically significant differences in the measured parameters they investigated. 
Our study provided baseline information on socio-demographic, behavioral/environmental, bio-physiological and emerging risk factors for CHD in three subgroups of a predominantly Black population in South Florida. In general, the findings of this study showed that the foreign-born Afro Caribbean (FBCA) individuals had a better behavioral health profile compared to their US-born counterparts. The FBCA had better behavioral (healthy diet as measured by the diet score, watching television [proxy for leisure-time inactivity], lower percentage being obese, lower percentages not smoking or drinking alcoholic beverages) profiles. The dietary findings included the observations that the FBCA participants compared to the USBCA and AA participants consumed significantly less total fat, saturated fat, percent energy from fat, fat servings, dietary cholesterol and sodium and more percent energy from carbohydrate and vegetables. A similar trend was seen for the FBCA males compared with the USBCA and AA males. For the FBCA females, only the mean dietary cholesterol intake was significant. Of interest, the mean total fat, saturated fat and dietary cholesterol intakes of the FBCA participants were significantly $(p<0.05)$ lower than the intakes reported for the African American population by the NHANES III study (Popkins et al., 2001) whereas the intakes of these nutrients for the USBCA and AA participants approximate that of the NHANES III values. Also, the percentages of participants exceeding dietary recommendations for percent energy from fat, saturated fat, cholesterol and sodium and not meeting these recommendations for fruit consumption were higher for USBCA and AA participants compared to the FBCA participants, with the differences being more pronounced in the male ethnic subgroups. The fat, dietary cholesterol, vegetable and 
sodium components of the diet are the major differences between the groups and indeed modifiable and subject to appropriate interventions.

Over the last decade the US Government (Healthy People 2010) and the American Heart Association (AHA Statistical Update, 2004) have set recommendations for improving the dietary intakes of the US population. The recommendations are to reduce total fat, especially saturated fat, dietary sodium and increase fruits, vegetables and fiber intakes. Although the knowledge of CHD risk factors was high in this population, the USBCA and AA participants in this study were apparently not following these recommendations. Maybe the messages were not culturally sensitive or specific to this ethnic group or, as reported by Anderson (2003) there may be differences in the social environment in which food is selected and consumed, areas not investigated in this study. Understanding the social context of food choice may be as important as identifying the food sources of the major nutrients in food (Anderson, 2003). This is because food provides a means to demonstrate cultural identity and is also symbolic of many areas of the participants' origin, religious background and beliefs structures (Anderson, 2003). We also observed that more USBCA and AA participants were obese compared to the FBCA participants. Other authors (Stephen et al., 1994; Singh and Siahpush, 2002; Hicks et al., 2003) also found that more US-born individuals were indeed obese compared to their foreign-born counterparts. The USBCA and AA participants may be at increased risk of CHD based on our findings that the percentages of USBCA and AA participants with behavioral and biophysical risk factors for $\mathrm{CHD}$ were higher than FBCA participants. These behavioral and biophysical risk factors for CHD have been associated 
with risk for CHD in numerous reports (NCEP: ATP III, 2001; AHA Statistical Update, 2004; Pearson et al., 2002).

These findings will become more noteworthy as the population of foreign-born individuals especially Afro Caribbeans increases. The need for culturally sensitive messages and programs to address health disparities by ethnically trained health care professionals will become even more important. It is also important that the health care establishment evaluates and understands the health needs, concerns and behavioral propensities of this and other ethnic groups.

According to the US Census Brief Report (2001), the term, "Black or African American" describes individuals having origins in any of the Black racial groups of Africa and, includes individuals who reported their race as "Black, African American, Negro, Afro American, Nigerian or Haitian," on the 2000 census survey. Such broad categorization of individuals does not take into consideration the existence of subgroups/cultures within this black population. These subgroups/cultures maybe composed of varying numbers of foreign-born individuals. Also, individuals of this black population may share some socio-demographic and genetic characteristics. Thus, differences in attitude, knowledge and behaviors leading to differences in some of the risk factors for CHD within the subcultures of this Black population (Kleier, 2003). Thus, it may be of vital importance to health policy planners and health care providers in the US, to monitor the health status of immigrants, especially as the population of immigrants increases (Stephen et al., 1994). The challenge of preventing CHD in this population lies in identifying and addressing the components of CHD most relevant to each ethnic group (Yusuf et al., 2001) especially dietary habits, one of the major 
differences between FBCA and the US-born counterparts. Poor dietary habit, one of the components of behavioral profile, is the cause of over $\$ 33$ billion in medical cost and over $\$ 9$ billion in lost productivity due to $\mathrm{CHD}$, cancer, stroke and diabetes mellitus (AHA Statistical Update, 2004).

Research is required to elucidate the various mechanisms for the differences in health behaviors between foreign- and US-born counterparts. Additionally research should examine screening for multiple behavioral and metabolic risk/risk factors in other racial/ethnic-cultural groups and subgroups with respect to geography (geographical transition of risks) and other behavioral factors such as sleep (Pronk et al., 2004), working at home/housework and visitations to healthcare professionals. Also, research on the behavioral activities of immigrants in the US versus their activities in their country of birth may help us better understand the geographical transition of behavioral and other risk factors for CHD in this and other ethnic populations. It is suggested that we should also investigate individuals who adhere to multiple health behavior recommendations as this might help us to understand the mechanisms that influence such behaviors (Berrigan et al., 2003). This might be helpful in the design of programs that will encourage healthier behaviors in this and other populations.

Focus should be placed on the ethnic and cultural differences in a population to better understand the variations in health indicators among different ethnic and cultural groups of the same race. This focus can provide healthcare professionals and policy planners with the opportunity to develop culturally sensitive nutrition/health programs and strategies for the improvement of health outcomes. 


\section{REFERENCES}

Albert CM, Ma J, Rifai N, Stampfer MJ, Ridker PM. Prospective Study of C-reactive Protein, Homocysteine and Plasma Lipid Levels as Predictors of Sudden Cardiac Death. Circulation 2002; 105: 2595-2599.

Albert MA, Glynn RJ, Ridker PM. Plasma Concentration of C-reactive Protein and the Calculated Framingham Coronary Heart Disease Risk Score. Circulation 2003; 108: 161-165.

American Diabetes Association. The Expert Committee on the Diagnosis and

Classification of Diabetes Mellitus. Clinical Practice Recommendations 2002. Diabetes Care 2002; 25(Suppl 1): S1-S147.

American Dietetic Association. Nutrition Experts Respond to New Government Report Card on American Diet. 1995; July 19: News Release.

American Heart Association. Heart and Stroke Statistical Update 2002. Dallas, TX: American Heart Association: 2001. www.americanheart.org. Accessed June 10, 2002.

American Heart Association. Heart and Stroke Statistical Update 2003. Dallas, TX: American Heart Association: 2002. www.americanheart.org. Accessed March 21, 2003.

American Heart Association. Heart and Stroke Statistical Update 2004. Dallas, TX: American Heart Association: 2003. www.americanheart.org. Accessed July 14, 2004.

Anand SS. Using Ethnicity as a Classification Variable in Health Research: Perpetuating the Myth of Biological Determinism, Serving Socio-political Agendas, or Making Valuable Contributions to Medical Sciences? Ethn Health 1999; 4: 241-244.

Anderson AS. The Challenge of Assessing Nutrient Intake in Ethnic Minority Groups. $\underline{J}$ Hum Nutr Dietet 2003; 16: 313-314.

Anderson LA, Eyler AA, Galuska DA, Brown DR, Brownson RC. Relationship of Satisfaction with Body Size and Trying to Lose Weight in a National Survey of Overweight and Obese Women aged 40 and Older, United States. Prev Med 2002; 35: 390-396.

Anderson JW, Hanna TJ. Impact of Non-digestible Carbohydrates on Serum Lipoproteins and Risk for Cardiovascular Disease. J Nutr 1999; 129: 1457-1466. 
Andreotti F, Kluft C. Circadian Variation of Fibrinolytic Activity in Blood. Chronobiol Int 1991; 8: 336-351.

Angleton P, Chandler WL, Schmer G. Diurnal Variation of Tissue-type Plasminogen Activator and its Rapid Inibition (PAI-1). Circulation 1989; 79: 101-106.

Assman G, Schulte H. Relation of High-density Lipoprotein Cholesterol and Triglycerides to Incidence of Atherosclerotic Coronary Disease (the PROCAM experience). Am J Cardiol 1992; 70: 733-737.

Assmann G, Schulte H, Funke H, von Eckardstein A. The Emergence of Triglycerides as a Significant Independent Risk Factor in Coronary Artery Disease. Eur Heart J 1998; 19(Suppl M): M8-M14.

Assmann G, Cullen P, Jossa F, Lewis B, Mancini M, for the International Task Force for the Prevention of Coronary Heart Disease. Coronary Heart Disease: Reducing the Risk. The Scientific Background to Primary and Secondary Prevention of Coronary Heart Disease. A World View. Arterioscler Thromb Vasc Biol 1999; 19: 1819-1824.

Atherosclerosis Risk in Communities Investigators. The Atherosclerosis Risk in Communities (ARIC) Study: Design and Objectives. Am J Epidemiol 1989; 129: 687-702.

Austin MA, Hokanson JE, Edwards KL. Hypertriglyceridemia as a Cardiovascular Risk Factor. Am J Cardiol 1998; 81: 7B-12B.

Austin MA, McKnight B, Edwards KL, Bradley CM, McNeely MJ, Psaty BM, Brunzell JD, Motulsky AG. Cardiovascular Disease Mortality in Familial Forms of Hypertriglyceridemia: A 20-year Propective Study. Circulation 2000a; 101: 27772782.

Baecke JAH, Burema J, Frijters JER. A Short Questionnaire for the Measurement of Habitual Physical Activity in Epidemiological Studies. Am J Clin Nutrition 1982; 36: 936-942.

Baum A, Garofalo JP, Yali A. Socio-conomic Status and Chronic Stress: Does Stress Account for SES Effects on Health? Annals of the New York Academy of Sciences 1999; 896: 131-144.

Beaglehole R, Saracci R, Panico S. Editorial. Cardiovascular Diseases: Causes, Surveillance and Prevention. Int J Epidemiol 2001; 30: S1-S4.

Barter PJ. Inhibition of Endothelial Cell Adhesion Molecule Expression by High-density Lipoproteins. Clin Exp Pharmacol Physiol 1997; 24: 286-287. 
Bauer, U. Diabetes in Florida. J Florida Med Assoc. 1998; 85: 20-21.

Bensen JT, Hutchinson RG, Province MA, Tyroler HA. Family History of Coronary Heart Disease and Pre-clinical Carotid Artery Atherosclerosis in AfricanAmericans and whites: The ARIC Study: Atherosclerosis Risk in Communities. Genet Epidemiol 1999; 16: 165-178.

Berrigan D, Dodd K, Troiano RP, Krebs-Smith SM, Barbash RB. Patterns of Health Behavior in US adults. Prev Med 2003; 36: 615-623.

Blair SN, Cooper KH, Gibbons LW, Gettman LR, Lewis S, Goodyear N. Changes in Coronary Heart Disease Risk Factors Associated with Increased Treadmill Time in 753 Men. Am J Epidemiol 1983; 118: 352-359.

Blair D, Habicht JP, Sims EA, Slywester D, Abraham S. Evidence for an Increased Risk for Hypertension with Centrally Located Body Fat and the Effect of Race and Sex on this Risk. Am J Epidemiol 1984; 119: 526-540.

Blake GJ, Ridker PM. Inflammatory Bio-markers and Cardiovascular Risk Prediction. J Int Med 2002; 252: 283-294.

Block G, Hartman AM, Dresser CM, Carroll MD, Gannon J, Gardner L. A Data-based Approach to Diet Questionnaire Design and Testing. Am J Epidemiol 1986; 124: 453-469.

Block G, Woods M, Potosky A, Clifford C. Validation of a Self-administered Diet History Questionnaire Using Multiple Diet Records. J Clin Epidemiol 1990; 43: 1327-1335.

Bonow RO, Eckel RH. Diet, Obesity and Cardiovascular Risk. N Eng J Med 2003; 348 : 2057-2058.

Boushey CJ, Beresford SAA, Omenn GS, Motulsky AG. A Quantitative Assessment of Plasma Homocysteine as a Risk Factor for Vascular Disease: Probable Benefits of Increasing Folic Acid Intakes. JAMA 1995; 274: 1049-1057.

Bowman SA, Lino M, Gerrior SA, Basiotis PP. The Healthy Eating Index: 1994-96. Washington DC: US Department of Agriculture, Center for Nutrition Policy and Promotion. 1998.

Braunwald E. Shattuck Lecture - Cardiovascular Medicine at the Turn of the Millennium: Triumphs, Concerns and Opportunities. New Eng J Med 1997; 337 : 1360-1369.

Bronner Y. Cultural Sensitivity and Nutrition Counseling. Topics in Clinical Nutrition 1994; 9: 13-19. 
Brookins GK. Culture, Ethnicity and Bicultural Competence: Implications for Children with Chronic Illness and Disability. Pediatr 1993; 91: 1056-1062.

Brown MS, Goldstein JL. A Receptor-mediated Pathway for Cholesterol Homeostasis. Science 1986; 232; 34-37.

Buchowski MS, Sun M. Energy Expenditure, Television Viewing and Obesity. Int J Obes 1996; 20: 236-244.

Budde T, Fechtrup C, Bosenberg E, Vielhauer C, Enbergs A, Schulte H, Assmann G, Breithardt G. Plasma Lp (a) Levels Correlate with Number, Severity, and Lengthextension of Coronary Lesions in Male Patients Undergoing Coronary Arteriography for Clinically Suspected Coronary Atherosclerosis. Arterioscler Thromb 1994; 14: 1730-1736.

Burchard EG, Ziv E, Coyle N, Gomez SL, Tang H, Karter AJ, Mountain JL, Perez-Stable EJ, Sheppard D, Risch N. The Importance of Race and Etnic Background in Biomedical Research and Clinical Practice. N Eng J Med 2003; 348: 1170-1175.

Buring JE, O'Connor GT, Goldhaber SZ, Rosner B, Herbert PN, Blum CB, Breslow JL, Hennekens CH. Decreased HDL2 and HDL3 Cholesterol, apo A-1 and apo A-II, and Increased Risk of Myocardial Infarction. Circulation 1992; 85: 22-29.

Cairney J, Ostbye T. Time Since Immigration and Excess Body Weight. Canadian J Public Health 1999; 90: 120-124.

Callaway CW, Chumlea WC, Bouchard C, Himes JH, Lohman TG, Martin AD, Mitchell CD, Mueller WH, Roche AF, Seefeldt VD. Measurement Descriptions and Techniques. In: Anthropometric Standardization Manual, Human Kinetics Books, 1988. A Division of Human Kinetics Publishers, Inc., P.O. Box 5075, Champaign, IL 61820.

Calle EE, Thun MJ, Petrelli JM, Rodriquez C, Heath CW. Body Mass Index and Mortality in a Cohort Study of US Adults. N Eng J Med 1999; 341: 1097-1105.

Calles-Escandon, J, Ballor D, Harvey-Berino J, Ades P, Tracy R, Sobel B. Amelioration of the Inhibition of Fibrinolysis in Elderly Obese Subjects by Moderate Energy Intake Restriction. Am J Clin Nutr 1996; 64: 7-11.

Campbell V. Caribbean Foodways. Caribbean Food and Nutrition Institute (CFNI) June, 1988; Kingston, Jamaica.

Cappuccio FP, Cook DG, Atkinson RW, Strazzullo P. Prevalence, Detection and Management of Cardiovascular Risk Factors in Different Ethnic Groups in South London. Heart 1997; 78: 555-563. 
Castelli WP, Garrison RJ, Wilson PW, Abbott RD, Kalousdian S, Kannel WB. Incidence of Coronary Heart Disease and Lipoprotein Cholesterol Levels: The Framingham Study. JAMA 1986; 256: 2835-2838.

Center for Disease Control \& Prevention. Folate Status in Women of Childbearing Age United States, 1999. MMWR 2000; 49: 962-965.

Center for Disease Control \& Prevention. 2000. Statistics: Prevalence of Diabetes. http://www.cdc.gov/diabetes/pubs/factsheet.htm. Assessed July 2, 2003.

Center for Disease Control \& Prevention. http://www.cdc.gov/omh/AMH/factsheets/cardio.htm. Assessed October 8, 2002.

Chave SP, Morris JN, Moss S, Semmence AM. Vigorous Exercise in Leisure Time and the Death Rate: A Study of Male Civil Servants. J Epidemiol Community Health 1978; 32: 239-243.

Clark LT, Ferdinand KC, Flack JM, Gavin JR III, Hall WD, Kumanyika SK, Reed JW, Saunders E, Valantine HA, Watson K, Wenger NK, Wright JT. Coronary Heart Disease in African-Americans. Heart Disease 2001; 3: 97-108.

Cohen J. Statistical Power Analysis for the Behavioral Sciences. $2^{\text {nd }}$ Edition, 1988. Lawrence Erlbaum Associates, Hillsdale, NJ.

Cohen S, Kamarck T, Mermelstein R. A Global Measure of Perceived Stress. J Health Social Behav 1983; 24: 385-396.

Cohen S, Williamson GM. Perceived Stress in a Probability Sample of the United States, in Spacapan SS, Oskamp S, eds., The Social Psychology of Health. Newbury Park, CA: Sage, 1988.

Colditz GA, Bonita R, Stampfer MJ, Willett WC, Rosner B, Speizer FE, Hennekens CH. Cigarette Smoking and Tisk of Stroke in Middle-aged Women. N Eng J Med 1988; 318: 937-941.

Cooper RS. Social Inequality, Ethnicity and Cardiovascular Disease. Int J Epidemiol 2001; 30: S48-S52.

Cooper RS, Rotimi CN, Kaufman JS, Owoaje EE, Fraser H, Forrester T, Wilks R, Riste LK, Cruickshank JK. Prevalence of NIDDIM among Populations of the African Diaspora. Diabetes Care 1997; 20: 343-348. 
Cooper R, Rotimi C, Ataman S, McGee D, Osotimehin B, Kadiri S, Muna W, Kingue S, Fraser H, Forrester T, Bennett F, Wilks R. The Prevalence of Hypertension in Seven Populations of West African Origin. Am J Public Health 1997a; 87: 160168.

Coups EJ, Gaba A, Orleans CT. Physician Screening for Multiple Behavioral Health Risk Factors. Am J Prev Med 2004; 27(Suppl 2): S34-S41.

Criqui MH, Golomb BA. Epidemiologic Aspects of Lipid Abnormalities. Am J Med 1998; 105(Suppl): 48S-57S.

Cruickshank JK, Mbanya JC, Wilks R, Balkau B, Forrester T, Anderson SG, Mennen L, Forhan A, Riste L, McFarlane-Anderson N. Hypertension in Four African-origin Populations: Current 'Rules of Halves', Quality of Blood Pressure Control and Attributable Risk of Cardiovascular Disease. J Hypertens 2001; 19: 41-46.

Curtis S, Lawson K. Gender, Ethnicity and Self-Reported Health: The Case of AfricanCaribbean Populations in London. Social Science and Medicine 2000; 50: 365385.

Cushman M, Meilhan EN, Psaty BM, Hormane Replacement Therapy, Inflammation, and Hemostasis in Elderly Women. Arterioscler Thromb Vasc Biol 1999; 19: 893-899.

Dallman MF, Strack AM, Akana SF, Bradbury MJ, Hanson ES, Scribner KA, Smith M. Feast and Famine: Critical Role of Glucocorticoids with Insulin in Daily Energy Flow. Font Neuroendocrinol 1993; 14: 303-347.

Dannenberg AI, Garrison RJ, William BK. Incidence of Hypertension in the Framingham Study. Am J Pub Health 1988; 78: 676-679.

Davey SG, Wentworth D, Neaton JD, Stamler R, Stamler J. Socioeconomic Differentials in Mortality Risk among Men Screened for the Multiple Risk Factor Intervention Trial: 2. Black Men. Am J Public Health 1996; 86: 497-504.

de Ferranti S, Rifai N. C-reactive Protein and Cardiovascular Disease: A Review of risk Predictive and Interventions. Clinica Chimica Acta 2002; 317: 1-15.

Denke MA, Sempos CT, Grundy SM. Excess Body Weight. An Under-recognized Contributor to High Blood Cholesterol Levels in White American Men. $\underline{\text { Arch }}$ Intern Med 1993; 153: 1093-1103. 
Diabetes Control and Complicatiions Trial Research Group (DCCT). The Effect of Intensive Treatment of Diabetes on the Development and Progression of Longterm Complications in Insulin-dependent Diabetes Mellitus. N Eng J Med 1993; 329: 977-986.

Dietz WH, Gortmaker SL. Do We Fatten Our Children at the Television Set? Obesity and Television Viewing in Children and Adolescents. Pediatrics 1985; 75: 807-812.

Downs JR, Clearfield M, Weis S, Whitney E, Shapiro DR, Beere PA, Langendorfer A, Stein EA, Kruyer W, Gotto AM. Primary Prevention of Acute Coronary Events with Lovastatin in Men and women with Average Cholesterol Levels: Results of AFCAPS/TexCAPS. JAMA 1998; 279: 1615-1622.

Dufour MC. If You Drink Alcoholic Beverages Do So in Moderation: What Does This Mean? J Nutr 2001; 131: 552-561.

Dundas R, Morgan M, Redfern J, Lemic-Stojcevic N, Wolfe C. Ethnic Differences in Behavioral Risk Factors for Stroke: Implications for Health Promotion. Ethn Health 2001; 6: 95-103.

Eckel RH. Obesity and Heart Disease: A statement for Healthcare Professionals from the Nutrition Committee, American Heart Association. Circulation 1997; 96: 32483250 .

Erhardt L, Hobbs FD. Public Perceptions of Cardiovascular Risk in Five European Countries: The React Survey. Int J Clin Pract 2002; 56: 638-644.

Executive Summary of the Third Report of the National Cholesterol Education Program (NCEP) Expert Panel on Detection, Evaluation and Treatment of High Blood Cholesterol in Adults (ATP III). JAMA 2001; 285: 2486-2497.

Fang J, Medhavan S, Alderman MH. The Association between Birthplace and Mortality from Cardiovascular Causes Among Black and White Residents of New York City. N Eng Med J 1996; 335: 1545-1551.

Fang J, Madhaven S, Alderman MH. Nativity, Race and Mortality: Favorable Impact of Birth Outside the United States on Mortality in New York City. Hum Biol 1997: 69: 689-701.

Festa A, D'Agostino R, Howard G, Mykkanen L, Tracy RP, Haffner SM. Chronic Subclinical Inflammation as Part of the Insulin Resistance Syndrome: The Insulin Resistance Atherosclerosis Study (IRAS). Circulation 2000; 102: 42-47. 
Fine LJ, Philogene G S, Gramling R, Coups EJ. Prevalence of Multiple Chronic Disease Risk Factors: 2001 National Health Interview Survey. Am J Prev Med 2004; 27(Suppl 2): S18-S24.

Flegal KM, Carroll MD, Ogden CL, Johnson CL. Prevalence and Trends in Obesity Among USA Adults, 1999-2000. JAMA 2002; 288: 1723-1727.

Fletcher GF, Balady G, Blair SN, Blumenthal J, Caspersen C, Chaitman B, Epstein S, Sivarajan J, Froelicher ES, Froelicher VF, Pina IL, Pollock ML. Statements on Exercise: Benefits and Recommendations for Physical Activity Programs for All Americans. A Statement for Health Professionals by the Committee on Exercise and Cardiac Rehabilitiation of the Council on Clinical Cardiology. American Heart Association. Circulation 1996; 94: 857-862.

Florida Cardiovascular Surveillance Summary and State Plan of Action. Florida Department of Health, Bureau of Chronic Disease Prevention. 2001; Tallahassee, FL 32399. www.doh.state.fl.us. Assessed August 12, 2003.

Folsom AR, Kaye SA, Sellers TA, Hong CP, Cerhan JR, Potter JD, Prineas RJ. Body Fat Distribution and 5-year Risk of Death in Older Women. JAMA 1993; 269: 483487.

Food Composition Tables for Use in the English-speaking Caribbean. Caribbean Food and Nutrition Institute (CFNI), 1995; Kingston, Jamaica.

Food Composition Tables for Use in the English-speaking Caribbean. Caribbean Food and Nutrition Institute (CFNI): Supplement, 2000, Kingston, Jamaica.

Ford CS. Body Mass Index, Diabetes and C-reactive Protein among US Adults. Diabetes Care 1999; 22: 1971-1977.

Ford ES, Ford MA, Will JC, Galuska DA, Ballew C. Achieving a Healthy Lifestyle Among United States Adults: A Long Way to Go. Ethn Dis 2001; 11: 224-231.

Ford ES. Does Exercise Reduce Inflammation? Physical Activity and C-reactive Protein among US Adults. Epidemiology 2002; 13: 561-568.

Ford, Giles WH, Dietz WH. Prevalence of the Metabolic Syndrome among US Adults: Findings from the Third National Health and Nutrition Examination Survey. JAMA 2002; 287: 356-359.

Frazao E. The American Diet: A Costly Health Problem. FoodReview 1996; 19: 2-6. 
Freidewald WT, Levy RI, Fredrickson DS. Estimation of the Concentration of Lowdensity Lipoprotein Cholesterol in Plasma without Use of the Centrifuge. Clin Chem 1972; 18: 499-502.

Fuster V, Lewis A. Conner Memorial Lecture. Mechanisms Leading to Myocardial Infarction: Insights from Studies of Vascular Biology. Circulation 1994; 90 : 2126-2146.

Gaines K, Burke G. Ethnic Differences in Stroke: Black-White Differences in the United States Population. Neuroepidemiol 1995; 14: 209-239.

Gardner CD, Fortmann SP, Krauss RM. Association of Small Low-density Lipoprotein Particles with the Incidence of Coronary Artery Disease in Men and Women. JAMA 1996; 276: 875-881.

Gates G, McDonald M. Comparison of Dietary Risk Factors for Cardiovascular Disease in African-Americans and White Women. J Am Diet Assoc 1997; 97: 1394-1400.

Gavin JR, Alberti KGMM, Davidson MB, DeFronzo RA, Drash A, Gabbe SG, Genuth S, Harris MI, Kahn R, Keen H, Knowler WC, Lobovitz H, Maclaren NK, Palmer JP, Raksin P, Rizza RA, Stern MP. Report of the Expert Committee on the Diagnosis and Classification of Diabetes Mellitus. Diabetes Care 1998; 21(suppl 1): S5-S19.

Genest JJ, McNamara JR, Salem DN, Schaefer EJ. Prevalence of Risk Factors in Men with Premature Coronary Artery Disease. Am J Cardiol 1991; 67: 1185-1189.

Gerber LM. The Influence of Environmental Factors on Mortality from Coronary Heart Disease among Filipinos in Hawaii. Hum Biol 1980; 52: 269-278.

Gerber LM, Madhaven S. Epidemiology of Coronary Heart Disease in Migrant Chinese Population. Med Anthropol 1980; 4: 307-320.

Gey KF, Moser UK, Jordan P, Stahelin HB, Eichholzer M, Ludin E. increased Risk of Cardiovascular Disease at Suboptimal Plasma Concentrations of Essential Antioxidants: An Epidemiological Update with Special Attention to Carotene and Vitamin C. Am J Clin Nutr 1993; 57(suppl): 787S-797S.

Giles WH, Croft JB, Greenlund KJ, Ford ES, Kittner SJ. Association between Total Homocysteine and the Likelihood for a History of Acute Myocardia; Infarction by Race and Ethnicity: Results from the Third National Health and Nutrition Examination Survey. Am Heart J 2000; 139: 446-453.

Gillum RF. Stroke in Blacks. Stroke 1988; 19: 1-9. 
Gillum RF, Mussolino ME, Madans JH. Coronary Heart Disease Incidence and Survival in African-American Women and Men. The NHANES I Epidemiologic Followup Study. Ann Intern Med 1997; 127: 111-118.

Gillum RF. Sudden Death in Hispanic Americans and African-Americans. Am J Public Health 1997; 87:1461-1466.

Glueck CJ, Gartside F, Fallart RW, Sielski J, Steiner PM. Longivity Syndromes: Familial Hypobeta- and Familial Hyperalphalipoproteinemia. J Lab Clin Med 1976; 88: 941-957.

Goldbourt U, Yaari S, Medalie JH. Isolated Low HDL Cholesterol as a Risk Factor for Coronary Heart Disease Mortality. A 21-year Follow-up of 8000 Men. Arterioscler Thromb Vasc Biol 1997; 17: 107-113.

Goldstein LB, Adams R, Becker K, Furberg CD, Gorelick PB, Hademenos G, Hill M, Howard G, Howard VJ, Jacobs B, Levine SR, Mosca L, Sacco RL, Sherman DG, Wolf PA, del Zoppo, GJ. Primary Prevention of Ischemic Stroke: A Statement for Healthcare Professionals from the Stroke Council of the American Heart Association. Stroke 2001; 32: 280-299.

Gordon DJ, Probstfield JL, Garrison RJ, Neaton JD, Castelli WP, Knoke JD, Jacobs DR Jr, Bangdiwala S, Tyroler HA. High-density Lipoprotein Cholesterol and Cardiovascular Disease: Four Prospective American Studies. Circulation 1989; 79: 8-15.

Gorelick PB. Cerebrovascular Disease in African-Americans. Stroke 1998; 29: 26562664.

Gortmaker SL, Dietz WH, Cheung LWY. Inactivity, Diet and the Fattening of America. J Am Diet Assoc 1990; 90: 1252-1255.

Gotto Jr AM, Whitney E, Stein EA, Shapiro DR, Clearfield M, Weis S, Jou JY, Langendörfer A, Beere PA, Watson DJ, Downs JR, de Cani JS. Application of the National Cholesterol Education Program and Joint European Treatment Criteria and Clinical Benefit in the Air Force/Texas Coronary Atherosclerosis Prevention Study (AFCAPS/TexCAPS). European Heart J 2000; 21: 1627-1633.

Greenberg M, Schneider D. Region of Birth of Blacks in the United States. Int J Epidemiol 1992: 21: 324-328.

Greenland P, Knoll MD, Stamler J, Neaton JD, Dyer AR, Garside DB, Wilson PW. Major Risk Factors as Antecedants of Fatal and Nonfatal Coronary Heart Disease Events. JAMA 2003; 290: 891-897. 
Grover SA, Coupal L, Hu XP. Identifying Adults at Increased Risk of Coronary Disease. How Well do the Current Cholesterol Guidelines Work? JAMA 1995; 274: 801806.

Grundy SM, Blackburn G, Higgins M, Lauer R, Perri MG, Ryan . Physical Activity in the Prevention and Treatment of Obesity and its Comorbidities: Evidence Report of Independent Panel to Assess the Role of Physical Activity in the Treatment of Obesity and its Comorbidities. Med Sci Sports Exerc 1999a; 31: 1493-1500.

Grundy SM, Pasternak R, Greenland P, Smith Jr. S, Fuster V. Assessment of Cardiovascular Risk by Use of Multiple-Risk Factor Assessment Equations. A Statement for Healthcare Professionals from the American Heart Association and the American College of Cardiology. Circulation 1999; 100: 1481-1492.

Gussekloo J, Schaap MC, Frolich M, Blauw GJ, Westendorp RG. C-reactive Protein is a Strong but Non-specific Risk Factor of Fatal Stroke in Elderly Persons. Arterioscler Thromb Vasc Biol 2000; 20: 1047-1051.

Han TS, van Leer EM, Seidell JC, Lean ME. Waist Circumference Action Levels in the Identification of Cardiovascular Risk Factors: Prevalence Study in a Random Sample. BM J 1995; 311: 1401-1405.

Hann SC, Rock CL, King I, Drewnowski A. Validation of the Healthy Eating Index with Use of Plasma Biomarkers in a Clinical Sample of Women. Am J Clin Nutr 2001; 74: 479-486.

Hansson L, Zanchetti A, Carruthers SG, Dahlöf B, Elmfeldt D, Julius S Menard J, Rahn $\mathrm{KH}$, Wedel H, Westerling S. Effects of Intensive Blood -pressure Lowering and Low-dose Aspirin in Patients with Hypertension: Principal Results of the Hypertension Optimal Treatment (HOT) Randomized Trial. HOT Study Group. Lancet 1998; 351: 1755-1762.

Harris, M. NIDDM: Epidemiology and Scope of the Problem. Diabetes Spectrum. 1996; 9: 26-29.

Harris KM. The Health Status and Risk Behaviors of Adolescents in Immigrant Families. In: Children of Immigrants: Health, Adjustments and Public Assistance. National Academy Press, Washington DC. 1999; 286-347. www.nap.edu/openbook/0309065453/html/286.html

Hartman A, Rundek T, Mast H, Paik MC, Boden-Albala B, Mohr JP, Sacco RL. Mortality and Causes of Death after First Ischemic Stroke. The Northern Manhattan Stroke Study. Neurology 2001; 57: 2000-2005. 
Haskell WL, Leon AS, Caspersen CJ, Froelicher VF, Hagberg JM, Harlan W, Holloszy JO, Regensteiner JG, Thompson PD, Wahburn RA, Wilson PWF. Cardiovascular Benefits and Assessment of Physical Activity and Physical Fitness in Adults. Med Sci Sports Exerc 1992; 24(Suppl 6): S201-S220.

Haskell WL, Alderman EL, Fair JM, Maron DJ, Mackey SF, Superko HR, William PT, Johnstone IM, Champagne MA, Krauss RM, Farquhar JW. Effects of Intensive Multiple Risk Factor Reduction on Coronary Atherosclerosis and Clinical Cardiac Events in Men and Women with Coronary Artery Disease: The Stanford Coronary Risk Intervention Project (SCRIP). Circulation 1994; 89: 975-990.

Hatano S. Experience from a Multi-center Stroke Register: A preliminary Report. Bull World Health Organ 1976; 54: 541-553.

Heald AH, Anderson SG, Ivison F, Laing I, Gibson JM, Cruickshank K. C-reactive Protein and the Insulin-like Growth Factor (IGF)-system in Relation to Risk of Cardiovascular Disease in Different Ethnic Groups. Atherosclerosis 2003; 170: 79-86.

Health Care Financing Administration (HCFA) [now Centers for Medicare and Medicaid Services (CMS)], Review Statistical Supplement, 2000.

Healthy People 2010: Objectives for Improving Health. Priority Area 12. Heart Disease and Stroke. http://www.health.gov. Assessed February 1, 2002.

Helmrich SP, Ragland DR, Leung RW, Paffenbarger RS Jr. Physical Activity and Reduced Occurrence of Non-insulin Dependent Diabetes Mellitus. N Eng J Med 1991; 325: 147-152.

Hemmingway H, Marmot, M. psychosocial Factors in the Aetiology and Prognosis of Coronary Heart Disease: Systematic Review of Prospective Cohort Studies. $\underline{B M J}$ 1999; 318: 1460-1467.

Hemmingway H, Whitty CJ, Shipley M, Stansfeld MS, Brunner E, Fuhrer R, Marmot M. Psychosocial Risk Factors for Coronary Disease in White, South Asian and AfroCaribbean Civil Servants: The Whitehall II Study. Ethn Dis 2001; 11: 391-400.

Hennekens CH, Dyken ML, Fuster V. Aspirin as a Therapeutic Agent in Cardiovascular Disease: A Statement for Healthcare professionals from the American Heart Association. Circulation 1997; 96: 2751-2753.

Henry FJ. obesity-Related Mortality, Morbidity and Behavior in the Caribbean. Cajanus 2001; 34: 62-72. 
Hernandez DJ ED. Children of Immigrants: Health, Adjustments and Public Assistance. Washington DC: National Academy of Sciences Press, 1999.

Hicks LS, Fairchild DG, Cook EF, Ayanian JZ. Association of Region of Residence and Immigrant Status with Hypertension, Renal Failure, Cardiovascular Disease and Stroke among African American Participants in the Third National Health and Nutrition Survey (NHANES III). Ethn Dis 2003; 13: 316-323.

Hopkins PN. Effects of Dietary Cholesterol on Serum Cholesterol: A Meta-analysis and Review. Am J Clin Nutr 1992; 55: 1060-1070.

Hu FB, Stampfer MJ, Manson JE, Rimm E, Colditz GA, Rosner BA, Hennekens CH, Willett WC. Dietary Fat Intake and the Risk of Coronary Heart Disease in Women. N Eng J Med 1997; 337: 1491-1499.

Hu FB,Stampfer MJ, Rimm EB, Manson JE, Ascherio A, Colditz GA, Rosner BA, Spiegelman D, Speizer FE, Sacks FM, Hennekens CH, Willett WC. A Prospective Study of Egg Consumption and Risk of Cardiovascular Disease in Men and Women. JAMA 1999; 281: 1387-1394.

Hu FB, Stampfer MJ, Colditz GA, Ascherio A, Rexrode KM, Willett WC, Manson JE. Physical Activity and Risk of Stroke in Women. JAMA 2000; 283: 2961-2967.

Huijbregts P, Feskens E, Rasanen L, Fidanza F, Nissinen A, Menotti A, Krombout D. dietary Pattern and 20 Year Mortality in Elderly Men in Finland, Italy, and the Netherlands: Longitudinal Cohort Study. BMJ 1997; 314: 13-17.

Hunt SC, Gwinn M, Adams TD. Family History Assessment: Strategies for Prevention of Cardiovascular Disease. Am J Prev Med 2003; 24: 136-142.

Hyman DJ, Simons-Morton DG, Ho K. Cholesterol-related Knowledge, Attitudes and Behaviors in a Low-income, Urban Patient Population. Am J Prev Med 1993; 9: 292-299.

Imhof A, Froehlich M, Brenner H, Boeing H, Pepys MB, Koenig W. Effect of Alcohol Consumption on Systemic Markers of Inflammation. Lancet 2001; 357: 763-767.

Institute of Medicine, Care without Coverage: Too Little, Too Late. Committee on the Consequences of Uninsurance. Board on Health Care Services. Institute of Medicine, 2002. National Academy Press, Washington, DC.

Jackson M. risk Factors for Obesity in Caribbean Women and Children. Cajanus 2001; 2: 88-96. 
Jacobsen BK, Thelle DS. Risk Factors for Coronary Heart Disease and Levels of Education. The Tromso Heart Study. Am J Epidemiol 1988; 127: 923927.

Jacques PF, Selhub J, Bostom AG, Wilson PW, Rosenberg IH. The Effect of Folic Acid Fortification on Plasma Folate and Total Homocysteine Concentrations. $\mathrm{N}$ Eng J Med 1999; 340: 1449-1454.

Jonas MA Oates JA, Ockene JK, Hennekens CH. Statement on Smoking and Cardiovascular Disease for Health Care Professionals. Circulation 1992; 86: 1664-1669.

Kagan A, Harris BR, Winkelstein W Jr, Johnson KG, Kato H, Syme SL, Rhoads GG, Gay ML, Nichaman MZ, Hamilton HB, Tillotson J. Epidemiologic Studies of Coronary Heart Disease and Stroke in Japanese Men Living in Japan, Hawaii and California: Demographic, Physical, Dietary and Biochemical Characteristics. J Chronic Dis 1974; 27: 345-364.

Kannel WB, Dawber TR, McGee DL. Perspectives on Systolic Hypertension, the Framingham Study. Circulation 1980; 61: 1179-1182.

Kannel WB. High-density Lipoproteins: Epidemiologic Profile of Risks of Coronary Artery Disease. Am J Cardiol 1983; 52: 9B-12B.

Kannel WB, D'Agostino RB, Sullivan L, Wilson PWF. Concept and Usefulness of Cardiovascular Risk Profiles. Am Heart J 2004; 148: 16-26.

Kaplan GA, Keil JE. Socio-economic Factors and Cardiovascular Disease: A Review of the Literature. Circulation 1993; 88: 1973-1998.

Katan MB. Trans Fatty Acids and Plasma Lipoproteins. Nutr Rev 2000; 58: 188-191.

Keil JE, Loadholt CB, Weinrich MC, Sandifer SH, Boyle Jr. Incidence of Coronary Heart Disease in Blacks in Charleston, South Carolina. Am Heart J 1984; 108: 779-786.

Khaw KT, Barrett-Connor E. Family History of Heart Attack: A Modifiable Risk Factor? Circulation 1986; 74: 239-244.

King H, Kriska AM. Prevention of Type 2 Diabetes by Physical Training: Epidemiological Considerations and Study Methods. Diabetes Care 1992; 15(Suppl 4): 1794-1799.

Kington RS, Smith JP. Socioeconomic Status and Racial and Ethnic Differences in Functional Status Associated with Chronic Diseases. Am J Public Health 1997; 87: 805-810. 
Kinosian B, Glick H, Preiss L, Puder KL. Cholesterol and Coronary Heart Disease: Predicting Risks in Men by Changes in Levels and Ratios. J Investig Med 1995; 43: 443-450.

Kittler PM, Sucher KP. Food and Culture. 3rd Edition; Wadworth/Thomson Learning Publishing, Belmont, CA; 2001.

Koenig W, Sund M, Frohlich M, Fischer HG, Lowel H, Doring A, Hutchinson WL, Pepys MB. C-Reactive Protein, a Sensitive Marker of Inflammation, Predicts Future Risk of Coronary Heart Disease in Initially Healthy Middle-Aged Men. Results from the MONICA (Monitoring Trends and Determinants in Cardiovascular Disease) Augsburg Cohort Study, 1984 to 1992. Circulation 1999; 99: 237-242.

Kostis JB, Davis BR, Cutler J, Grimm RH Jr, Berge KG, Cohen JD, Lacy CR, Perry HM Jr, Blaufox MD, Wassertheil-Smoller S, Black HR, Schron E, Berkson DM, Curb JD, Smith WM, McDonald R, Applegate WB. Prevention of Heart Failure by Antihypertensive Drug Treatment in Older Persons with Isolated Systolic Hypertension. Systolic Hypertension in the Elderly Program (SHEP) Cooperative Research Group. JAMA 1997; 278: 212-216.

Krauss RM, Eckel RH, Howard B, Appel LJ, Daniels SR, Deckelbaum RJ, Erdman JW Jr, Kris-Etherton P, Goldberg IJ, Kotchen TA, Lichtenstein AH, Mitch WE, Mullis R, Robinson K, Wylie-Rosett J, St. Jeor S, Suttie J, Tribble DL, Bazzarre TL. AHA Dietary Guidelines: Revision 2000: A Statement for Healthcare Professionals from the Nutrition Committee of the American Heart Association. Circulation 2000; 102: 2284-2299.

Kris-Etherton PM. Monounsaturated Fatty Acids and Risk of Cardiovascular Disease. Circulation 1999; 100: 1253-1258.

Kriska AM, Caspersen CJ. Baecke Questionnaire of Habitual Physical Activity. Med Sci Sports Exerc 1997; 29(Suppl 6): S15-S18.

Kumanyika SK. Diet and Nutrition as Influences on the Morbidity/Mortality Gap. Ann Epidemiol 1993; 3: 154-158.

Kuller LH, Tracy RP, Shaten J, Meilahn EN (for the Multiple Risk Factors Intervention Trial [MRFIT] Research Group). Relationship of C-Reactive Protein and Coronary Heart in the MRFIT Nested Case-Controlled Study. Am J Epidemiol 1996; 144: 537-547.

LaCroix AZ, Lang J, Scherr P, Wallace RB, Cornoni-Huntley J, Berkman L, Curb JB, Evans D, Hennekens CH. Smoking and Mortality among Older Men and Women in Three Communities. N Eng J Med 1991; 324: 1619-1625. 
LaVeist TA, Bowie JA, Cooley-Quille M. Minority Health Status in Adulthood: The Middle Years. Health Care Financing Review 2000a; 21: 1-13.

LaVeist TA, Bowie J, Nickerson K. Attitudes, about Racism, Medical Mistrust and Satisfaction with Care among African American and White Cardiac Patients. Medical Care Research and Review 2000b; 57: 147-161.

Law MR, Wald NJ, Thompson SG. By How Much and How Quickly does Reduction in Serum Cholesterol Concentration Lower Risk of Ischemic Heart Disease? BMJ 1994b; 308: 367-372.

Law MR. Lowering Heart Disease Risk with Cholesterol Reduction: Evidence from Observational Studies and Clinical Trials. Eur Heart J Suppl 1999; (Suppl 1): S3S8.

Ledue TB, Weiner DL, Sipe J, Poulin SE, Collins MF, Rifai N. Analytical Evaluation of Particle-enhanced Immunoephelometric Assays for C-reactive Protein, Serum Amyloid A and Monnose-binding Protein in Human Serum [Abstract]. Clin Chem 1997; 43: S240.

Leon AS, Myers MJ, Connett J. Leisure-time Physical Activity and the 16-year risk of mortality from Coronary Heart Disease and all-causes in the Multiple Risk Factor Intervention Trial (MRFIT). Int J Sports Med 1997; 18(Supp1): S208-S215.

Lemic-Stojcevic N, Dundas R, Jenkins S, Rudd A, Wolfe C. Preventable Risk Factors for Coronary Heart Disease and Stroke amongst Ethnic Groups in London. Ethn Health 2001; 6: 87-94.

Levy AS, Fein SB, Stephenson M. Nutrition Knowledge Levels about Dietary Fat and Cholesterol: 1983-1988. J Nutr Educ 1993; 25: 60-66.

Li R, Bensen JT, Hutchinson RG, Province MA, Hertz-Picciotti I, Sprafka JM, Tyroler HA. Family Risk Score of Coronary Heart Disease (CHD) as a Predictor of CHD: The Atherosclerosis Risk in Communities (ARIC) Study and the NHLBI Family Heart Study. Genet Epidemiol 2000; 18: 236-250.

Libby P, Ridker PM, Maseri A. Inflammation and Atherosclerosis. Circulation 2002; 105: 1135-1143.

Lindahl B, Weinehall L, Asplund K, Hallmans G. Screening for Impaired Glucose Tolerance. Results from a Population-based Study in 21,057 Individuals. Diabetes Care 1999; 22: 1988-1992.

Linton MF, Fazio S. A Practical Approach to Risk Assessment to Prevent Coronary Artery Disease and Its Complications. Am J Cardiol 2003; 92(suppl): 19i-26i. 
Lipid Research Clinics Program. The Lipid Research Clinics Coronary Primary

Prevention Trial Results, I: Reduction in the Incidence of Coronary Heart

Disease. JAMA 1984a; 251: 351-364.

Lipid Research Clinics Program. The Lipid Research Clinics Coronary Primary Prevention Trial Results, II: The Relationship of Reduction in Incidence of

Coronary Heart Disease to Cholesterol Lowering. JAMA 1984b; 251: 365-374.

Liu S, Stampfer MJ, Hu FB, Giovannucci E, Rimm E, Manson JE, Hennekens CH, Willett WC. Whole-grain Consumption and Risk of Coronary Heart Disease: Results from the Nurses' Health Study. Am J Clin Nutr 1999; 70: 412-419.

Logan JR, Deane G. Black Diversity in Metropolitan America. Lewis Mumford Center For Comparative Urban and Regional Research, University at Albany. August 15 , 2003.

Lowe GD, Yarnell JW, Rumley A, Bainton D, Sweetnam PM. C-reactive Protein, Fibrin D-dimer, and Incident Ischemic Heart Disease in the Speedwell Study: Are Inflammation and Fibrin Turnover Linked in Pathogenesis? Arterioscler Thromb Vasc Biol 2001; 21: 603-610.

Luepker RV, Rosamond WD, Murphy R, Sprafka JM, Folsom AR, McGovern PG, Blackburn H. Socio-economic Status and Coronary Heart Disease Risk Factor Trends. The Minnesota Heart Survey. Circulation 1993; 88: 2172-2179.

Mackness MI, Durrington PN. HDL, Its Enzymes and Its Potential to Influence Lipid Peroxidation. Atherosclerosis 1995; 115: 243-253.

Macy EM, Hayes TE, Tracy RP. Variability in the Measurement of C-reactive Protein in Healthy Subjects: Implications for Reference Intervals and Epidemiological Application. Clin Chem 1997; 43: 52-58.

McBride PE. The Health Consequences of Smoking. Cardiovascular Diseases. Med Clin North Am 1992; 76: 333-353.

McKeigue PM, Shah B, Marmot MG. Relation of Central Obesity and Insulin Resistance with High Diabetes Prevalence and Cardiovascular Risk in South Asians. Lancet 1991; 337: 382-386.

McMurray RG, Ainsworth BE, Harrell JS, Griggs TR, Williams OD. Is Physical Activity or Aerobic Power More Influential on Reducing Cardiovascular Disease Risk Factors? Med Sci Sports Exerc 1998; 30: 1521-1529.

McNamara JR, Schaefer EJ. Automated Enzymatic Standardized Lipid Analysis for Plasma and Lipoprotein Fractions. Clin Chim Acta 1987; 166: 1-8. 
Magnus P, Beaglehole R. The Real Contribution of the Major Risk Factors to the Coronary Epidemics: Time to End the "Only 50\%" Myth. Arch Intern Med 2001; 161: 2657-2660.

Malinow MR. Plasma Homocyst(e)ine - A Risk Factor for Arterial Occlusive Diseases. $\underline{J}$ Nutr 1996; 126(suppl): 1238S-1243S.

Malinov MR, Bostom AG, Krauss RM. Homocysteine, Diet and Cardiovascular Diseases. A Statement for Healthcare Professionals from the Nutrition Commmittee, American Heart Association. Circulation 1999; 99: 178-182.

Marin P, Anderson B, Ottosson M, Olbe L, Chowdhury B, Kvist H, Holm G, Sjostrom L, Bjorntorp P. The Morphology and Metabolism OF Intraabdominal Adipose Tissue In Men. Metabolism 1992; 41: 1242-1248.

Martin MJ, Hulley SB, Browner WS, Kuller LH, Wentworth D. Serum Cholesterol, Blood Pressure, and Mortality: Implications from a Cohort of 361,662 Men. Lancet 1986; 2: 933-936.

Mbanya JC, Cruickshank JK, Forrester T, Balkau B, Ngogang JY, Riste L, Forhan A, Anderson NM, Bennett F, Wilks R. Standardized Comparison of Glucose Intolerance in West African Origin Populations of Rural and Urban Cameroon, Jamaica and Caribbean Migrants to Britain. Diabetes Care 1999; 22: 434-440.

Mendell MA, Strachan DP, Butland BK, Ballam L, Morris J, Sweetnam PM, Elwood PC. C-Reactive Protein: Relation to Total Mortality, Cardiovascular Mortality and Cardiovascular Risk Factors in Men. Eur Heart J 2000; 21: 1584-1590.

Mensink RP, Katan MB. Effects of Dietary Fatty Acids on Serum Lipids and Lipoproteins. A Meta-analysis of 27 Trials. Arterioscler Thromb 1992; 12: 911919.

Millen BE, Quatromoni PA, Franz MM, Epstein BE, Cupples LA, Copenhafer DL. Population Nutrient Intake Approaches Dietary Recommendations: 1991-1995 Framingham Nutrition Studies. J Am Diet Assoc 1997; 97: 742-749.

Miller BD, Alderman EL, Haskell WL, Fair JM, Krauss RM. Predominance of Dense Low-density Lipoprotein Particles Predicts Angiographic Benefit of Therapy in the Stanford Coronary Risk Intervention Project. Circulation 1996; 94: 21462153.

Mokdad AH, Marks JS, Stroup DF, Gerberding JL. Actual Causes of Death in the United States, 2000. JAMA 2004; 291: 1238-1245. 
Montalescot G, Collet JP, Choussat R, Thomas D. Fibrinogen as a Risk Factor for Coronary Heart Disease. Eur Heart J 1998; 19(Suppl H): H11-H17.

Morbidity and Mortality Weekly Report (MMWR), 1999; 48: CDC/NCHS.

Morbidity and Mortality Weekly Report (MMWR), 2000; 49: CDC/NCHS.

Morbidity and Mortality Weekly Report (MMWR), 2004; 53: CDC/NCHS.

Muntner P, He J, Chen J, Fonseca V, Whelton PK. Prevalence of Non-traditional Cardiovascular Disease Risk Factors among Persons with Impaired Fasting Glucose, Impaired Glucose Tolerance, Diabetes and the Metabolic Syndrome: Analysis of the Third National Health and Nutrition Examination Survey (NHANES III). Ann Epidemiol 2004; 14: 686-695.

Natarajan S, Glick H, Criqui M, Horowitz D, Lipsitz SR, Kinosian B. Cholesterol Measures to Identify and Treat Individuals at Risk for Coronary Heart Disease. Am J Prev Med 2003; 25: 50-57.

National Center for Health Statistics: Plan and Operation of the Third National Health and Examination Survey. Gaithersburg: DHHS Pub. No (PHS) 94-1308, 1994.

National Cholesterol Education Program. Third Report of the Expert Panel on Detection, Evaluation, and Treatment of High Blood Cholesterol in Adults (Adult Treatment Panel [ATP] III, 2001). Also available at www.nhlbi.nih.gov/guidelines/cholesterol/atp3 rpt.htm. Assessed April 1, 2002.

National Health and Nutrition Examination Survey III (NHANES III, 1988-1994). Prevalence Estimates for Coronary Heart Disease, Stroke and Congestive Heart Failure. $\mathrm{CDC} / \mathrm{NCHS}$.

National Heart, Lung and Blood Institute, National Institute of Health. Fact Sheets on Black Americans, Hispanic Americans, Asian/Pacific Islanders, and American Indians. May, 1992.

National Heart, Lung and Blood Institute, Healthy Heart I.Q. NIH Publication No. 932724. www.nhlbi.nih.gov/health/public/heart/other/hh-iq.htm. Accessed August 9, 2002.

National Heart, Lung, and Blood Institute (NHLBI). Morbidity and Mortality:1998 Chart-book on cardiovascular, Lung and Blood Diseases. Bethesda, MD: Public Health Service (PHS), National Institutes of Health (NIH), NHLBI, October, 1998. 
National Heart, Lung and Blood Institute, Test Your Cholesterol and Heart Disease I.Q. NIH Publication No. 95-3794. www.nhlbi.nih.gov/health/public/chol/chol-iq.htm. Accessed August 9, 2002.

National Institute of Health (NIH). Physical Activity and Cardiovascular Health: NIH Consensus Statement, 1995; 13: 1-33.

National Institutes of Health (NIH). Clinical Guidelines on the Identification, Evaluation and Treatment of Overweight and Obesity in Adults: Evidence Report. NIH Pub. No. 98-4083. Bethesda, MD: National Heart, Lung and Blood Institute, 1998a.

National Institutes of Health (NIH). Clinical Guidelines on the Identification, Evaluation and Treatment of Overweight and Obesity in Adults: Evidence Report. J Obesity Res 1998b; 6(Suppl. 2): 51S-209S.

Newman TB, Browner WS, Cummings SR, Hulley SB. Designing an Observational Study: Cross-sectional and Case-control Studies. In: Designing Clinical Research. An Epidemiological Approach, $2^{\text {nd }}$ Edition; 2001. Williams and Wilkins, Philadelphia.

Nicklas TA, Webber LS, Srinivasan SR, Berenson GS. Secular Trends in Dietary Intakes and Cardiovascular Risk Factors of 10 year Old Children: The Bogalusa Heart Study (1973-1988). Am J Clin Nutr 1993; 57: 930-937.

Niggs CR, Allegrante JP, Ory MG. Theory-comparison and Multiple-behavior Research: Common Themes Advancing Health Behavior Research. Health Educ Res 2002; 17: $670-679$.

Nikkila M, Koivula T, Niemela K, Sisto T. High Density Lipoprotein Cholesterol and Triglycerides as Markers of Angiographically assessed Coronary Artery Disease. Br Med J 1990; 63: 78-81.

Ockene IS, Miller NH. Cigarette Smoking, Cardiovascular Disease and Stroke. Circulation 1997; 96: 3243-3247.

Orleans CT, Gruman J, Ulmer C, Emont SL, Hollendonner JK. Rating Our Progress in Population Health Promotion: Report Card on Six Behaviors. Am J Health Promot 1999; 14: 75-83.

Orleans, CT. Addressing Multiple Behavioral Health Risks in Primary Care. Am J Prev Med 2004; 27(Supp1 2): S1-S3 
Packard CJ, O'Reilly DS, Caslake MJ, McMahon AD, Ford I, Cooney J, MacPhee CH, Suckling KE, Krishna M, Wilkinson FE, Rumley A, Lowe GD. Lipoproteinassociated Phospholipase A2 as an Independent Predictor of Coronary Heart Disease. West of Scotland Coronary Prevention Study Group. N Eng J Med 2000; 343: 1148-1155.

Pate RR, Pratt M, Blair SN, Haskell WL, Macera CA, Bouchard C, Buchner D, Ettinger W, Heath GW, King AC, Kriska A, Leon AS, Marcus BH, Morris J, Pattenbarger RS, Patrick K, Pollock ML, Rippe JM, Sallis J, Wilmore JH. Physical Activity and Public Health. A Recommendation from the Centers for Disease Control and Prevention and the American College of Sports Medicine. JAMA 1995; 273: 402407.

Pearson TA. Alcohol and Heart Disease. From the National Committee of the American Heart Association. Circulation 1996; 94: 3023-3025.

Pearson TA, Mensah GA, Alexander RW, Anderson JL, Cannon RO III, Criqui M, Fadl YY, Fortmann SP, Hong GL, Myers GL, Rifai N, Smith SC Jr, Taubert K, Tracy RP, Vinicor F. Markers of Inflammation and Cardiovascular Disease: Application to Clinical and Public Health Practice: A Statement for Healthcare Professionals from the Centers of Disease Control and Prevention and the American Heart Association. Circulation 2003; 107: 499-511.

Pepys MB, Baltz ML. Acute Phase Proteins with Specific Reference to C-reactive Protein and Related Proteins (Pentaxins) and Serum Amyloid A Protein. Adv Immunol 1983; 34: 141-212.

Pepys MB, Hirschfield GM. C-reactive Protein: A Critical Update. J Clin Invest 2003; 111: $1805-1812$.

Perry IJ, Refsum H, Morris RW, Ebrahim SB, Ueland PM, Shaper AG. Prospective Study of Serum and Total Homocysteine Concentration and Risk of Stroke in Middle-aged British Men. Lancet 1995; 346: 1395-1398.

Perseghin G, Price TB, Petersen KF, Roden M, Cline GW, Gerow K, Rothman DL, Shulman GI. Increased Glucose Transport-phosphorylation and Muscle Glycogen Synthesis after Exercise Training in Insulin-resistant subjects. N Eng J Med 1996; 335: 1357-1362.

Phillips RL, Lilienfeld AM, Diamond EL, Kagan A. Frequency of Coronary Heart Disease and Cerebrovascular Accidents in Parents and Sons of Coronary Heart Disease Index Cases and Controls. Am J Epidemiol 1974; 100: 87-100. 
Pietinen P, Rimm EB, Korhonen AM, Willett WC, Albanes D, Virtamo J. Intake of Dietary Fiber and Risk of Coronary Heart Disease in a Cohort of Finnish Men. The Alpha-Tocopherol, Beta-carotene Cancer Prevention Study. Circulation 1996; 94: 2720-2727.

Plawecki H. Cultural Considerations. J Holistic Nurs 1992; 10: 4-5.

Pohjola-Sintonen S, Rissanen A, Liskola P, Luomanmaki K. Family History as a Risk Factor of Coronary Heart Disease in Patients under 60 Years of Age. Eur Heart J 1998; 19: 235-239.

Pomerleau J, McKeigue PM, Chaturvedi N. Factors Associated with Obesity in South Asian, Afro-Caribbean and European Women. Int J Obes Relat Disord 1999; 23: 25-33.

Pooling Project Research Group. Relationship of Blood Pressure, Serum Cholesterol, Smoking Habit, Relative Weight and ECG Abnormalities to Incidence of Major Coronary Events: Final Report of the Pooling Project. J Chronic Dis 1978; 31 : 201-306.

Popkin BM. The Nutrition Transition in Low Income Countries: An Emerging Crisis. Nutr Rev 1994; 52: 285-298.

Popkin BM, Siega-Riz AM, Haines PS, Jahns L. Where's the Fat? Trends in US Diets 1965-1996. Prev Med 2001; 32: 245-254.

Pradham AD, Manson JE, Rossouw JE, Siscovick DS, Mouton CP, Rifai N, Wallace RB, Jackson RD, Pettinger MB, Ridker PM. Inflammatory Biomarkers, Hormone Replacement Therapy and Incident Coronary Heart Disease: Prospective Analysis from the Women's Health Initiative Observational Study. JAMA 2002; 288: 980987.

Pronk NP, Anderson LH, Crain AL, Martinson BC, O’Connor PJ, Sherwood NE, Whitebird RR. Meeting Recommendations for Multiple Healthy Lifestyle Factors: Prevalence, Clustering and Predictors Among Adolescents, Adults and Senior Health Plan Members. Am J Prev Med 2004; 27(Suppl 2): S25-S33.

Pyörälä K, De Backer G, Graham I, Poole-Wilson P, Wood D. Prevention of Coronary Heart Disease in Clinical Practice: Recommendations of the Task Force of the European Society of Cardiology, European Atherosclerosis Society and European Society of Hypertension. Atherosclerosis 1994; 110: 121-161.

Retterstol L, Eikvar L, Berg K. A Twin Study of C-reactive Protein Compared to Other Risk Factors for Coronary Heart Disease. Atherosclerosis 2003; 169: 279-282. 
Ridker PM, Cushman M, Stampfer MJ, Tracy RP, Hennekens CH. Inflammation, Aspirin and the Risk of Cardiovascular Disease in Apparently Healthy Men. N Eng J Med 1997; 336: 973-979.

Ridker PM, Cushman M, Stampfer MJ, Tracy RP, Hennekens CH. Plasma Concentration of C-Reactive Protein and Risk of Developing Peripheral Vascular Disease.

Circulation 1998a; 97: 425-428.

Ridker PM, Buring JE, Shih J, Matias M, Hennekens CH. Prospective Study of CReactive Protein and the Risk of Future Cardiovascular Events among Apparently Healthy Women. Circulation 1998b; 98: 731-733.

Ridker PM, Glynn RJ, Hennekens CH. C-Reactive Protein Adds to the Predictive Value of Total and HDL Cholesterol in Determining Risk of First Myocardial Infarction. Circulation 1998c; 97: 2007-2011.

Ridker PM, Rifai N, Pfeffer MA, Sacks FM, Moye LA, Goldman S, Inflammation, Pravastatin and the Risk of Coronary Events after Myocardial Infarction in Patients with Average Cholesterol Levels. Cholesterol and Recurrent Events (CARE) Investigators. Circulation 1998d; 98: 839-844.

Ridker PM. Evaluating Novel Cardiovascular Risk Factors: Can We better Predict Heart Attacks? Ann Intern Med 1999; 130: 933-937.

Ridker PM, Hennekens CH, Rifai N, Buring JE, Manson JE. Hormone Replacement Therapy and Increased Plasma Concentration of C-reactive Protein. Circulation 1999b; 100: 713-716.

Ridker PM, Hennekens CH, Buring JE, Rifai N. C-Reactive Protein and other Markers of Inflammation in the Prediction of Cardiovascular Disease in Women. $\underline{\mathrm{N} \text { Eng J }}$ Med 2000; 342: 836-843.

Ridker PM, Rifai N, Clearfield M, Downs JR, Weis SE, Miles JS, Gotto AM Jr. Measurement of C-reactive Protein for the Targeting of Statin Therapy in the Primary Prevention of Acute Coronary Events. N Eng J Med 2001; 344: 19591965.

Ridker PM, Rifai N, Rose L, Buring JE, Cook NR. Comparison of C-reactive Protein and Low-density Lipoprotein Cholesterol Levels in the Prediction of First Cardiovascular Events. N Eng J Med 2002; 347: 1557-1565.

Ridker PM. Clinical Application of C-reactive Protein for Cardiovascular Disease Detection and Prevention. Circulation 2003; 107: 363-369. 
Ridker PM. High-sensitivity C-reactive Protein and Cardiovascular Risk: Rationale for

Screening and Primary Prevention. Am J Cardiol 2003a; 92(suppl): 17K-22K.

Ridker PM, Rifai N, Rose L, Buring JE, Cook NR. Comparison of C-reactive Protein and Low-density Lipoprotein Cholesterol Levels in the Prediction of First Cardiovascular Events. N Eng J Med 2003; 347(20): 1557-1565.

Rifai N, Tracy RP, Ridker PM. Clinical Efficacy of an Automated High-Sensitivity CReactive Protein Assay. Clin Chem 1999; 45: 2136-2141.

Rifai N, Ridker PM. High-Sensitivity C-Reactive Protein: A Novel and Promising Marker of Coronary Heart Disease. Clin Chem 2001; 47: 403-411.

Rifai N, Ridker PM. Proposed Cardiovascular Risk Assessment Algorithm Using Highsensitivity C-reactive Protein and Lipid Screening. Clin Chem 2001a; 47: 2830 .

Rifai N, Ridker PM. Inflammatory Markers and Coronary Heart Disease. Curr Opin Lipidol 2002; 13: 383-389.

Rimm EB, Ascherio A, Giovannucci E, Spiegelman D, Stampfer MJ, Willett WC. Vegetable, Fruit and Cereal Fiber Intake and Risk of Coronary Heart Disease among Men. JAMA 1996a; 275: 447-451.

Rimm EB, Klatsky A, Grobbee D, Stampfer MJ. Review of Moderate Alcohol Consumption and Reduced Risk of Coronary Heart Disease: Is the Effect due to Beer, Wine or Spirits? BMJ 1996b; 312: 731-736.

Rimm EB, Willett WC, Hu FB, Sampson L, Colditz GA, Manson JE, Hennekens CH, Stampfer MJ. Folate and Vitamin $\mathrm{B}_{6}$ from Diet and Supplements in Relation to Risk of Coronary Heart Disease among Women. JAMA 1998; 279: 359-364.

Rimm EB, Williams P, Fosher K, Criqui M, Stampfer MJ. Moderate Alcohol Intake and Lower Risk of Coronary Heart Disease: Meta-analysis of Effects on Lipids and Hemostatic Factors. BMJ 1999; 319: 1523-1528.

Rissanen AM, Nikkila EA. Coronary Artery Disease and its Risk Factors in Families of Young Men with Angina pectoris and in Controls. Br Heart J 1977; 39: 875-883.

Rissanen AM. Familial Aggregation of Coronary Heart Disease in a High Incidence Area (North Karelia, Finland). Br Heart J 1979; 42: 294-303. 
Robertson TL, Kato H, Gordon T, Kagan A, Rhoads GG, Land CE, Worth RM, Belsky JL, Dock DS, Miyanishi M, Kawamoto S. Epidemiologic Studies of Coronary Heart Disease and Stroke in Japanese Men Living in Japan, Hawaii and California: Coronary Heart Disease Risk Factors in Japan and Hawaii. Am J Cardiol 1977; 39: 244-249.

Rodriquez BL, Curb D, Burchfiel CM, Abbott RD, Petrovitch H, Masaki K, Chiu D. Physical Activity and 23-year Incidence of Coronary Heart Disease Morbidity and Mortality among Middle-aged Men. Circulation 1994; 89: 2540-2544.

Rohde LEP, Hennekens CH, Ridker PM. Survey of C-Reactive Protein and Cardiovascular Risk Factors in Apparently Healthy Men. Am J Cardiol 1999; 84: 1018-1022.

Roivainen M, Viik-Kajander M, Palosuo, T, Toivanen P, Leinonen M, Saikku P, Tenkanen L, Manninen V, Hovi T, Manttari M. Infections, Inflammation and the Risk of Coronary Heart Disease. Circulation 2000; 101: 252-257

Romm PA, Green CE, Reagan K, Rackley CE. Relation of Serum Lipoprotein Cholesterol Levels to Presence and Severity of Angiographic Coronary Artery Disease. Am J Cardiol 1991; 67: 479-483.

Rosamond WD, Folsom AR, Chambless LE, Wang CH, McGovern PG, Howard G, Cooper LS, Shahar E. Stroke Incidence and Survival among Middle-aged Adults: Nine-year Follow-up of the Atherosclerosis Risk in Communities (ARIC) Cohort. Stroke 1999; 30(4): 736-743.

Rosenberg IH. Homocysteine, Vitamins and Arterial Occlusive Disease - An Overview. J Nutr 1996; 126(suppl): 1235S-1237S.

Rosengren A, Wilhelmsen L, Eriksson E, Risberg B, Wedel H. Lipoprotein (a) and Coronary Heart Disease: A Prospective Case-control Study in a General Population Sample of Middle-aged Men. BMJ 1990; 301: 1248-1251.

Rosenson RS, Koening W. Utility of Inflammatory Markers in the Management of Coronary Artery Disease. Am J Cardiol 2003: 92(Suppl): 10i-18i.

Rosmond R. Roles of Stress in the Pathogenesis of the Metabolic Syndrome. Psychoneuroendocrinology 2005; 30: 1-10. www.elsevier.com/locate/psyneuen. Assessed 09/21/04.

Rost NS, wolf PA, Kase CS, Kelly-Hayes M, Silbershatz H, Massaro JM, D'Agostino RB, Franzblau C, Wilson PW. Plasma Concentration of C-reactive Protein and Risk of Ischemic Stroke and Transient Ischemic Attack: The Framingham Study. Stroke 2001; 32: 2575-2579. 
Rubins HB, Robins SJ, Collins D, Iranmanesh A, Wilt TJ, Mann D, Mayo-Smith M, Faas FH, Elam MB, Rutan GH, Anderson JW, Kashyap ML, Schectman G.

Distribution of Lipids in 8500 Men with Coronary Artery Disease. Department of Veterans Affair HDL Intervention Trial Study Group. Am J Cardiol 1995; 75: 1196-1201.

Saito M, Ishimitsu T, Minami J, Ono H, Ohrui M, Matsuoka H. Relations of Plasma Creactive Protein to Traditional Cardiovascular Risk Factors. Atherosclerosis 2003; 167: 73-79.

Sakkinen P, Abbott RD, Curb JD, Rodriguez BL, Yano K, Tracy RP. C-reactive Protein and Myocardial Infarction. J Clin Epidemiol 2002; 55: 445-451.

Scandinavian Simvastatin Survival Study Group. Randomized Trial of Cholesterol Lowering in 4444 Patients with Coronary Heart Disease: The Scandinavian Simvastatin Survival Study (4S). Lancet 1994; 344: 1383-1389.

Selhub J, Jacques PF, Wilson PW, rush D, Rosenberg IH. Vitamin Status and Intake as Primary Determinants of Homocysteinemia in an Elderly Population. JAMA 1993; 270: 2693-2698.

Sempos CT, Cleeman JI, Carroll MD, Johnson CL, Backorik PS, Gordon DJ, Burt VL, Briefel RR, Brown CD, Lippel K, Rifkind BM. Prevalence of High Blood Cholesterol among US Adults: An Update Based on Guidelines from the second Report of the National Cholesterol Education Progrram Adult Treatment Panel. JAMA 1993; 269: 3009-3014.

Seman LJ, DeLuca C, Jenner JL, Cupples LA, McNamara JR, Wilson PW, Castelli WP, Ordovas JM, Schaefer EJ. Lipoprotein (a) Cholesterol and Coronary Heart Disease in the Framingham Heart Study. Clin Chem 1999; 45: 1039-1046.

Sharma S, Cade J, Riste L, Cruickshank K. Nutrient Intake Trends among AfricanCaribbeans in Britain: A Migrant Population and its Second Generation. $\underline{\text { Public }}$ Health Nutr 1999; 2: 469-476.

Sharrett AR, Sorlie PD, Chambless LE, Folsom AR, Hutchinson RG, Heiss G, Szklo M. Relative Importance of Various Risk Factors for Asymptomatic Carotid Atherosclerosis versus Coronary Heart Disease Incidence. The Atherosclerosis Risk in Communities Study. Am J Epidemiol 1999; 149: 843-852.

Shaw A, Davis C. The Dietary Guidelines Focus on Reducing Excessive Intakes. FoodReview 1994; 17: 4-7. 
Sheperd J, Cobbe SM, Ford I, Isles CG, Lorimer AR, MacFarlane PW, McKillop JH, Packard CJ (for the West of Scotland Coronary Prevention Study Group).

Prevention of Coronary Heart Disease with Pravastatin in Men with

Hypercholesterolemia. N Eng J Med 1995: 333: 1301-1307.

Simons LA, Friedland Y, McCallum J, Simons J, Powell I, Heller R, Berry G. The Dubbo Study of the Health of Elderly: Correlates of Coronary Heart Disease at Study Entry. J Am Geriatr Soc 1991; 39: 584-590.

Singh GK, Siahpush M. Ethnic-immigrant Differentials in Health Behaviors, Morbidity and Cause-specific Mortality in the United States: An Analysis of Two National Databases. Hum Biol 2002; 74: 83-109.

Sinha DP. Food, Nutrition and Health in the Caribbean: A Time for Re-examination 1995. Caribbean and Nutrition Institute Press, Kingston, Jamaica.

Smith JK, Dykes R, Douglas JE, Krishnaswamy G, Berk S. Long-term Exercise and Atherogenic Activity of Blood Mononuclear Cells in Persons at Risk of Developing Ischemic Heart Disease. JAMA 1999; 281: 1722-1727.

Snowden CB, McNamara PM, Garrison RJ, Feinleib M, Kannel WB, Epstein FH. Predicting Coronary Heart Disease in Siblings - A Multivariate Assessment. The Framingham Heart Study. Am J Epedemiol 1982; 115: 217-222.

Solomon S, Kington R. National Efforts to Promote Behavior-change Research: Views from the Office of Behavioral and Social Sciences Research. Health Educ Res 2002; 17: 495-499.

Sonnenberg LM, Quatromoni PA, Gagnon DR, Cupples LA, Franz MM, Ordovas JM, Wilson PWF, Schaefer EJ, Millen BE. Diet and Plasma Lipids in Women. II. Macronutrients and Plasma Triglycerides, High-density Lipoprotein and the Ratio of Total to High-density Lipoprotein Cholesterol in Women: The Framingham Nutrition Studies. J Clin Epidemiol 1996; 49: 665-672.

Stamler J, Wentworth D, Neaton JD. Is the Relationship between Serum Cholesterol and Risk of Premature Death from Coronary Heart Disease Continuous and Graded? Findings in 356,222 Primary Screenees of the Multiple Risk Factor Intervention Trial (MRFIT). JAMA 1986; 256: 2823-2828.

Stamler J, Stamler R, Neaton JD, Wentworth D, Daviglus ML, Garside D, Dyer AR, Liu $\mathrm{K}$, Greenland P. Low-risk Factor Profile and Long-term Cardiovascular and Non-cardiovascular Mortality and Life Expectancy: Findings for 5 Large Cohorts of Young Adults and Middle-aged Men and Women. JAMA 1999; 282: 2012-2018. 
Stampfer MJ, Hu FB, Manson JE, Rimm EB, Willett WC. Primary Prevention of CHD in Women through Diet and Lifestyle. N Eng J Med 2000; 343: 16-22.

Stephen EH, Foote K, Hendershot GE, Schoenborn CA. Health of the Foreign-born Population: United States, 1989-90. Adv Data 1994;241: 1-12.

Stern MP, Haffner SM. Body Fat Distribution and Hyperinsulinemia as Risk Factors for Diabetes and Cardiovascular Disease. Arteriosclerosis 1986; 6: 123-130.

Stevens J, Kumanyika SK, Keil JE. Attitudes towards Body Size and Dieting: Differences Between Elderly Black and White Women. Am J Public Health 1994; 84: 1322-1325.

Stewart JA, Dundas R, Howard S, Rudd AG, Wolfe CDA. Ethnic Differences in Incidence of Stroke: Prospective Study with Stroke Register. BMJ 1999; 318: 967-971.

Stubbs P, Seed M, Lane D, Collinson P, Kendall F, Noble M. Lipoprotein (a) as a Risk Predictor for Cardiac Mortality in Patients with Acute Coronary Syndromes. Eur Heart J 1998; 19: 1355-1364.

Subar AF, Thompson FE, Kipnis V, Midthune D, Hurwitz P, McNutt S, McIntosh A, Rosenfeld S. Comparative Validation of the Block, Willett and National Cancer Institute Food Frequency Questionnaires: The Eating at America's Table Study. Am J Epidemiol 2001; 154: 1089-1099.

Sugatani J, Miwa M, Komiyama Y, Ito S. High-density Lipoprotein Inhibits the Synthesis of Platelet-activating Factor in Human Vascular Endothelial Cells. J Lipid Mediat Cell Signal 1996; 13: 73-88.

Susuf S, Reddy S, Ounpuu S, Anand S. Global Burden of Cardiovascular Diseases Part 1: General Considerations, the Epidemiologic Transition, Risk Factors, and Impact of Urbanization. Circulation 2001; 104: 2746-2753.

Systolic Hypertension in the Elderly Program (SHEP) Cooperative Research Group. Prevention of Stroke by Antihypertensive Drug Treatment in Older Persons with Isolated Systolic Hypertension. Final Results of the SHEP. JAMA 1991; 265: 3255-3264.

Tate JR, Rifai N, Berg K, Couderc R, Dati F, Kostner GM, International Federation of Clinical Chemistry Standardization Project for the Measurement of Lipoprotein (a), Phase 1. Evaluation of the Analytical Performance of Lipoprotein (a) Assay Systems and Commercial Calibrators. Clin Chem 1998; 44: 1629-1640. 
The Sixth Report of the Joint National Committee (JNC VI) on Prevention, Detection, Evaluation, and Treatment of High Blood Pressure. Arch Intern Med 1997; 157: 2413-2446.

The Seventh Report of the Joint National Committee (JNC VII) on Prevention, Detection, Evaluation, and Treatment of High Blood Pressure. JAMA 2003; 289(19): 25602572.

Tisi PV, Hulse M, Chulakdabba A, Gosling P, Shearman CP. Exercise Training for Intermittent Claudication: Does it Adversely Affect Biochemical Markers of the Exercise-induced Inflammatory Response? Eur J Vasc Endovasc Surg 1997; 14: 344-350.

Toth PP. High-density Lipoprotein and Cardiovascular Risk. Circulation 2004; 109: 1809-1812.

Tracy RP, Lemaitre RN, Psaty BM, Ives DG, Evans RW, Cushman M, Meilahn EN, Kuller LH. Relationship of C-reactive protein to Risk of Cardiovascular Disease in the Elderly: Results from the Cardiovascular Health Study and the Rural Health Promotion Project. Arterioscler Thromb Vasc Biol 1997a; 17: 1121-1127.

Tracy RP, Psaty BM, Macy E, Bovill EG, Cushman M, Corrnell ES, Kuller LH. Lifetime Smoking Exposure Affects the Association of C-reactive Protein with Cardiovascular Disease in Risk Factors and Subclinical Disease in Healthy Elderly Subjects. Arterioscler Thromb Vasc Biol 1997b; 17: 2167-2176.

Tripathy D, Carlsson M, Almgren P, Isomaa B, Taskinen MR, Tuomi T, Groop LC. LDL Cholesterol. Insulin Secretion and Insulin Sensitivity in Relation to Glucose Tolerance: Lessons from the Botnia Study. Diabetes Care 2000; 49: 975-980.

Tyroler HA, Kowles MG, Wing SB, Loque EE, Davis CE, Heiss G, Heyden S, Hames CG. Ischemic Heart Disease Risk Factors and Twenty-year Mortality in Middleaged Evans County Black Males. Am Heart J 1984; 108: 738-746.

UK Prospective Diabetes Study (UKPDS) Group. Tight Blood Pressure Control and Risk of Macrovascular and Microvascular Complications in Type 2 Diabetes: (UKPDS 38). BMJ 1998d; 317: 703-713.

US Department of Agriculture. USDA Nutrient Database for Standard Reference. www.nal.usda.gov/fnic/foodcomp. Accessed August 9, 2002.

US Department of Agriculture and US Department of Health and Human Services. Nutrition and Your Health: Dietary Guidelines for Americans. $5^{\text {th }}$ edition. Home and Garden Bulletin no. 232. Washington, D.C.: US department of Agriculture 2000; 87 pages. 
US Census Bureau, Current Population Reports, Series P23-206, Profile of the Foreign-born Population in the United States: 2000, US Government Printing Office, Washington, DC, 2001.

http://www.census.gov/population/www/socdemo/foreign.htlm. Accessed March $8,2002$.

US Census Bureau, Coming from the Americas; A Profile of the Nation's Foreign-born Population from Latin America (2000 Update), Census Brief: Current Population Survey, 2002. http://www.census.gov/population/www/socdemo/foreign.htlm. Accessed March 31, 2003.

US Census Bureau, The Foreign-born Population in the United States: March 2002, Current Population Reports, P20-539, US Census Bureau, Washington, DC, 2003. http://www.census.gov/population/www/socdemo/foreign.htlm. Accessed March 31, 2003.

US census Bureau, Poverty 2002.

http://www.census.gov/hhes/poverty/threshld/thresh02.htm. Accessed May 16, 2003.

US Census Bureau, Department of Commerce, Economic and Statistics Administration, 2001. www.census.gov/prod/2001pubs/cenbr01-1.pdf. Accessed March 31, 2003.

US Department of Health and Human Services. Public Health Services. Healthy People 2000. National Health Promotion \& Disease Prevention Objectives. DHHS Publication No. (PHS) 91-50213. Washington D.C. US Government Printing Office, 1991.

US Department of Health and Human Services. Physical Activity and Health: A Report of the Surgeon General. Atlanta, Georgia: US Department of Health and Human Services, Public Health Service, Center for Disease Control and Prevention, National Center for Chronic Disease Prevention and Health Promotion, 1996b.

US Surgeon General. The Health Benefits of Smoking Cessation: A Report of the Surgeon General. DHHS pub. No. (CDC) 90-8416. Washington, DC.: US Department of Health and Human Services, 1990.

US Department of Agriculture, Human Nutrition Information Service. The Food Guide Pyramid. Washington, DC: USDA, 1992.

US Department of Agriculture/US Department of Health and Human Services. Nutrition and Your Health: Dietary Guidelines for Americans. Washington, DC: USDA/USDHHS, 1990. 
US Department of Agriculture, Agricultural Research Service, Data Tables: Results from USDA's 1994-1996 Continuing Survey of Food Intakes by Individuals and 19931996 Diet and Health Knowledge Survey (onlinre). ARS Food Surveys Research Group. Available (under releases @): http://www.barc.usda.gov/bhnrc/fooddsurvey/home.htm., Accessed October 8, 2001.

US Bureau of the Census, Population Projections Program, Population Division (2000, Jan). Projections of the Resident Population by Race, Hispanic Origin, and Nativity: Middle Series, 1999 and 2000. Washington, DC: US Bureau of the Census.

http://www.census.org/population/projections/nation/summary/np-t5-a.txt. Accessed October 8, 2001.

US Department of Agriculture, Human Nutrition Information Source. Primary Nutrient Data Set for USDA Food Consumption Surveys. 1998; Release 14, USDA, Springfield, VA.

Vasan RS, Larson MG, Leip EP, Kannel WB, Levy D. Assessment of Frequency of Progression to Hypertension in Non-hypertensive Participants in the Framingham Heart Study. Lancet 2001; 358: 1682-1686.

Vigushin DM, Pepys MB, Hawkins PN. Metabolic and Scintigraphic Studies of Radioiodinated Human C-reactive Protein in Health and Disease. J Clin Invest 1993; 91: 1351-1357.

Visser M, Bouter LM, McQuillan GM, Wener MH, Harris TB. Elevated C-reactive Protein Levels in Overweight and Obese Adults. JAMA 1999; 282: 2131-2135.

Verhoef P, Hennekens CH, Malinow MR, Kok FJ, Willett WC, Stampfer MJ. A Prospective Study of Plasma Homocysteine and Risk of Ischemic Stroke. Stroke 1994; 25: 1924-1930.

Verschuren VMM, Jacobs DR, Bloemberg BP, Kromhout D, Menoti A, Avantis C, Blackburn H, Buzina R, Dontas AS. Serum Total Cholesterol and Long-term Coronary Artery Disease Mortality in Different Cultures: Twenty-five-year Follow-up of the Seven Countries Study. JAMA 1995; 274: 131-136.

Wener MH, Daum PR, McQuillan GM. The Influence of Age, Sex, and Race on the Upper Reference Limit of Serum C-reactive Protein Concentration. J Rheumatol 2000; 27: 2351-2359.

White WB, Anwar YA. Evaluation of the overall Efficacy of the Omron Office Digital Blood Pressure HEM-907 Monitor in Adults. Blood Press Monit 2001; 6: 107110. 
Whitty CJM, Brunner EJ, Shipley MJ, Hemmingway H, Marmot MG. Differences in Biological Risk Factors for Cardiovascular Disease between Three Ethnic Groups in the Whitehall II Study. Atherosclerosis 1999; 142: 279-286.

Wilkinson CE, Goldman JD, Cook A. Trend in Food and Nutrient Intakes by Adults: NFCS 1977-78; CSFII 1989-91; CSFII 1994-95. Family Econ and Nutr Rev 1997; 10: 2-15.

Willett WC, Sampson L, Stampfer MJ, Rosner B, Bain C, Witschi J, Hennekens CH, Speizer FE. Reproducibility and Validity of a Semi-quantitative Food Frequency Questionnaire. Am J Epidemiol 1985; 122: 51-65.

Willett WC, Manson JE, Stampfer MJ, Colditz GA, Rosner B, Speizer FE, Hennekens $\mathrm{CH}$. Weight, Weight Change and Coronary Heart Disease in Women: Risk within the 'Normal' Weight Range. JAMA 1995; 273: 461-465.

Willett WC. Nutritional Epidemiology. Monographs in Epidemiology and Biostatistics, 1998; 30: 414-466. Oxford University Press, Inc, New York, NY 10016.

Williams DR. Black-White Differences in Blood Pressure: The Role of Social Factors. Ethn Dis 1992; 2: 126-141.

Williams RR, Hunt SC, Schumacher MC, . Genes, Hypertension and Coronary Heart Disease: Evidence for Shared Metabolic Pathophysiology. In: Smith U, Bruun NE, Hedner T, Hokfelt B, eds. Hypertension - An insulin-resistant Disorder. Genetic Factors and Cellular Mechanisms. Armsterdam: Elsevier, 1991; 89-101.

Williams RR, Hunt SC, Heiss G, Province MA, Bensen JT, Higgins M, Chamberlain RM, Ware J, Hopkins PN. Usefulness of Cardiovascular Family History Data for Population-based Preventive Medicine and Medical Research (the Health Family Tree Study and the NNHLBI Family Heart Study). Am J Cardiol 2001; 87: 129135.

Williamson AR, Hunt AE, Pope JR, Tolman NM. Recommendation of Dietitians for Overcoming Barriers to Dietary Adherence in Individuals with Diabetes. Diabetes Educator. 2000; 26: 272-279.

Wilson PW, D'Agostino RB, Levy D, Belanger AM, Silbershatz H, Kannel WB. Prediction of Coronary Heart Disease using Risk Factor Categories. Circulation 1998; 97: 1837-1847.

Wong ND, Pio J, Valencia R, Thakal G. Distribution of C-reactive Protein and its Relation to Risk Factors and Coronary Heart Disease Risk Estimation in the National Health and Nutrition Examination Survey (NHANES) III. Prev Cardiol 2001; 4: 109-114. 
Wood D, De Backer G, Faergeman O, Graham I, Mancia G, Pyörälä K. Prevention of Coronary Heart Disease in Clinical Practice: Recommendations of the Second Joint Task Force of European and other Societies on Coronary Prevention. Atherosclerosis 1998; 140: 199-270.

World Health Organization (WHO) Expert Committee on Biological Standardization. WHO Technical Report Series 760. Geneva, Switzerland; 1987.

World Health Organization (WHO). The World Health Report 2000. Health Systems: Improving Performance. Geneva, Switzerland: World Health Organization; 2000.

World Health Organization (WHO) Consultation on Obesity. Obesity: Preventing and Managing the Global Epidemic. WHO Technical Report Series 894. Geneva, Switzerland: World Health Organization; 2000b.

World Health Organization/Food and Agricultural Organization Expert Consultation Study Group on Diet, Nutrition and the Prevention of Chronic Diseases. WHO Technical Report Series 916. Geneva, Switzerland; 2002.

Yu H, Rifai N. High-sensitivity C-reactive Protein and Atherosclerosis: From Theory to Therapy. Clin Biochem 2000; 33: 601-610.

Yudkin JS, Stehouwer CD, Emeis JJ, Coppack SW. C-reactive Protein in Healthy Subjects: Associations with Obesity, Insulin Resistance and Endothelial Dysfunction: A Potential Role for Cytokines Originating from Adipose Tissue? Arterioscler Thromb Vasc Biol 1999; 19: 972-978.

Zoratti R. A Review on Ethnic Differences in Plasma Triglycerides and High-density Lipoprotein Cholesterol: Is the Lipid Pattern the Key Factor for the Low Coronary Heart Disease Rate in People of African Origin? Eur J Epidemiol 1998; 14: 9-21. 


\section{APPENDICES}

Appendix A: Recruitment Flyer

Appendix B: Informed Consent Form

Appendix C: Socio-demographic Questionnaire

Appendix D: Cohen Perceived Stress Questionnaire

Appendix E: Knowledge of CHD risk factors Questionnaire

1. Check your healthy Heart I.Q.

2. Check your Cholesterol and Heart Disease I.Q.

Appendix F: Food Frequency Questionnaire

Appendix G: Baecke Physical Activity Questionnaire

Appendix H: Document to Collect and Record

1. Weight

2. Height

3. Waist Circumference

4. Blood Pressure

5. Blood Lipids

6. High-sensitivity C-reactive Protein

Appendix I: Glossary of Health and Cultural Terms 
APPENDIX A 


\section{Florida International University \\ College of Health and Urban Affairs}

Comparison of Coronary Heart Disease Risk Factors in Caribbean-American and African-American Adults in South Florida

$>$ Aged $18-40$ Years Old?

Are You:

$>$ Caribbean National?

$>$ Caribbean-American?

$>$ African-American?

$>$ Not taking anti-inflammatory drugs (Aspirin, Motrin, Advil)?

$>$ Then You Can Participate in a Research Study

You will be asked to:

$>$ Sign an informed consent form.

$>$ Answer a dietary questionnaire.

$>$ Answer a cardiovascular risk questionnaire.

$>$ Answer a knowledge of heart disease risk questionnaire.

$>$ Answer a stress assessment questionnaire.

$>$ Give 1 tablespoon of blood.
You will Receive:

$>$ Meal voucher

$>$ Free study-related dietary assessment

$>$ Free study-related heart disease assessment

$>$ Free study-related assessment of heart disease knowledge

$>$ Free study-related stress assessment

For more information Call:

Errol Davis at 305-348-6427

Email: edavis02@fiu.edu

Room HLS 430 
APPENDIX B 


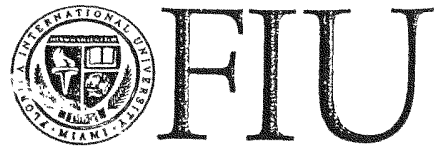

FLORIDA INTERNATIONAL UNIVERSITY

Miamis public research university
IRB Approvd

Date: $2211 / 03 \bigcirc$

No.: $010803-00$

\section{INFORMED CONSENT}

Comparison of Coronary Heart Disease Risk Factors in Caribbean-American and African-American Adults in South Florida

\section{PURPOSE}

You are being invited to freely participate in this study to look at risk factors for coronary heart disease among Caribbean-Americans and African-Americans. You will be one of one hundred and eighty individuals who will be involved in this study. The entire study will require 45-60 minutes of your time.

\section{PROCEDURES}

a You will be asked to complete five questionnaires that ask about (a) yourself and your family history of coronary heart disease and diabetes, (b) your food intake, (c) your physical activity, (d) your perceived stress and (e) your knowledge of coronary heart disease risk factors.

- Your weight, height and waist circumference will be measured

- Your blood pressure will be taken

a. One tablespoon (approximately $15 \mathrm{~mL}$ ) of blood will be taken, from your arm, by a licensed Phlebotomist, using standard procedures. This is necessary to measure your coronary heart disease risk. Some of your blood will be stored at $-70 \mathrm{oC}$, in one of our freezers, for future analysis of C-reactive protein (a marker for cardiovascular disease and inflammation) and other biochemical substances as needed.

\section{RISKS}

There is no cost to you for being in this study. You may feel a slight discomfort lasting less than a minute, due to the withdrawal of blood. However, with the use of sterile equipment and, standard procedures administered by a well-trained phlebotomy technician, the risk of soreness or infection is very small.

\section{REIMBURSEMENT}

You will receive a meal voucher ( $\$ 3.20$ value) which will be honored by the Pollo Tropical Restaurant, within a one month period, at Florida International University.

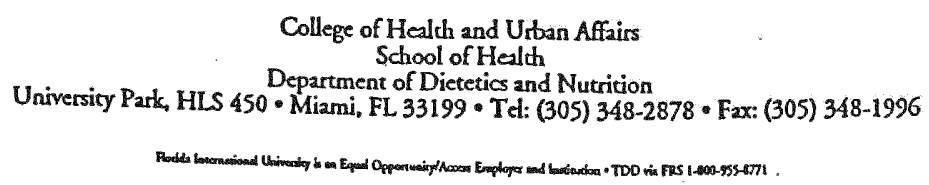




\section{CONFIDENTIALITY}

Your answers and other personal information on the survey forms will be kept strictly private. You will be given an identification number known ONLY to the people doing the study. All survey forms and other information taken from you will be kept in locked files that will be used only by the people doing the study.

\section{OTHER INFORMATION}

You are free to withdraw your consent and refuse further participation in this study at any time with no problems. You may ask questions about any materials and tests being used. We will provide answers to your satisfaction.

If you have more questions about this study, you may contact Dr. Fatma Huffman at (305) 3483788 or Mr. Errol Davis at (305) 348-6427. If you have any questions about your rights, you may call the Chairperson of the Institutional Review Board at Florida International University, Dr. Bernard Gerstman at (305) 348-3115. You will receive a copy of this Informed Consent form once you and the researcher have signed it.

I have read and understood the information above.

$\overline{\text { Participant's Name }}$

$\overline{\text { Participant's Signature }}$

\section{Date}

I have explained the study to the satisfaction of the participant and have provided a copy of this Informed Consent Form. 
APPENDIX C 


\section{Florida International University \\ Socio-demographic Questionnaire}

Dear Participant: Please answer the following questions about your lifestyle habits and please write or place a check $(\sqrt{ })$ in the appropriate spaces.

Pin \#

Last Name:

1 -Gender: 1 Male

2-Age: 1 _ less than 21 years

$\begin{array}{r}2 \\ 3\end{array}-31-30$ years

3 - Race and ethnic background

1 - African American
2 - Caribbean National/American
Date:

First Name:

2 Female

\section{4 - Please state your place of birth}

5 - How long have you lived in the United States of America?

$$
\begin{aligned}
& 1 \text { - less than } 1 \text { year } \\
& 2-1-3 \text { years } \\
& 3-4-5 \text { years }
\end{aligned}
$$

6 - Marital Status

\section{Single (never married)}

3 _ Separated or Divorced
4 4
5
$6 \_$__

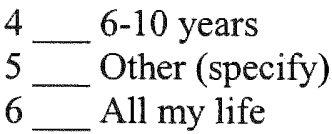

7 - At present, are you attending school?

_ Yes

No

If yes,

$$
\begin{aligned}
& 1 \text { full time } \\
& 2 \text { _ part-time } \\
& 3 \text { _ not attending school }
\end{aligned}
$$

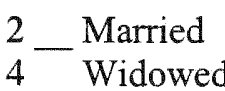

\section{8 - Education (completed)}

1 - $6^{\text {th }}$ grade (Caribbean)

3 _ High School (Caribbean)

5 - College (Caribbean)

7 - Graduate Studies (Caribbean)

9 - Other (Specify)

$2 \ldots 8^{\text {th }}$ grade (US)

4 High School (US)

6 College (US)

8 G_ Graduate Studies (US)

If yes, please specify

$$
9 \text { - Do you have medical insurance? }
$$

1 No

$$
2
$$
Yes
A _ Medicare
B Medicaid
C _ Private Provider Organization
D _ Home Management Organization

E Other (Specify) 
10 - How often do you visit a Health Care Professional for medical examination?

5 _ never/no visit in past year

1 Less than $\$ 10,000$

2 - $\$ 10,000-\$ 19,999$

3 - $\$ 20,000-\$ 29,999$

12 - Household Income

$$
\begin{aligned}
& 4 \text { - } \$ 30,000-\$ 39,000 \\
& 5 \text { more than } \$ 40,000
\end{aligned}
$$

13 - Monthly Expenditure on Food

1 Less than $\$ 100$
$2-\$ 100-\$ 200$
$3-\$ 200-\$ 300$

$4-\$ 300-\$ 400$

$5-\$ 400-\$ 500$

6 More than $\$ 500$

14 - How many individuals live in your household?

1 Children less than 5 years

3 Adults 21-64 years
2 Children $5-20$ years

4 Adults 65 years and older

15 - Family history of heart disease (check first correct answer):

1 two relatives with heart disease before 60 years of age

2 _ one relative with heart disease before 60 years of age

3 _ two relatives with heart disease after 60 years of age

4 _ one relative with heart disease after 60 years of age

5 - you have no family history of heart disease

6 _ you do not know your family history of heart disease

16 - Personal history of heart disease:

1 _ you have had or have been diagnosed to have heart disease

2 __ you have never had heart disease

17 - Family history of diabetes (check first correct answer):

1 _ you have two relatives with diabetes

2 - you have one relative with diabetes

3 _ you have no family history of diabetes

4 _ you do not know your family history of diabetes

18 - Personal History of diabetes

1 _ you have diabetes that began before the age of 20

2 _ you have diabetes that began between the ages $20-60$

3 _ you do not have diabetes

Please answer the other attached questionnaires

THANK YOU. 


\section{APPENDIX D}




\section{Florida International University \\ Cohen Perceived Stress Scale}

Pin \#

The questions in this scale ask you about your feeling and thoughts and perceived level of stress during the past month. Please circle a number for each question according to the following scale.

0 = Never $\quad 1$ =Almost Never $\quad 2$ =Sometimes 3 = Fairly Often 4 = Very Often

1. In the last month, how often have you been upset because of something that happened unexpectedly?

$[0] \quad[1] \quad[2] \quad[3] \quad[4]$

2. In the last month, how often have you felt that you were unable to control the important things

$[0] \quad[1] \quad[2] \quad[3]$ in your life?

3. In the last month, how often have you felt nervous and "stress"?

$[0] \quad[1] \quad[2] \quad[3]$

*4. In the last month, how often have you dealt successfully with irritating life hassles?

$[0] \quad[1] \quad[2] \quad[3] \quad[4]$

*5. In the last month, how often have you felt that you were effectively coping with important changes that were occurring in your life?

*6. In the last month, how often have you felt confident about your ability to handle your $[0] \quad[1] \quad[2] \quad[3] \quad[4]$ personal problems?

*7. In the last month, how often have you felt things were going your way?

$[0] \quad[1] \quad[2] \quad[3] \quad[4]$

8. In the last month, how often have you found that you could not cope with all the things that you had to do?

*9. In the last month, how often have you been able to control irritations in your life?

$[0] \quad[1] \quad[2] \quad[3] \quad[4]$

* 10. In the last month, how often have you felt that you were on top of things?

$[0] \quad[1] \quad[2] \quad[3] \quad[4]$

11. In the last month, how often have you been angered because of things that happened that were outside of your control? 
12. In the last month, how often have you found yourself thinking about things that you have

$[0] \quad[1] \quad[2] \quad[3] \quad[4]$ to accomplish?

*13. In the last month, how often have you been able to control the way you spend your time?

$[0] \quad[1] \quad[2] \quad[3] \quad[4]$

14. In the last month, how often have you felt that difficulties were piling up so high that you could not overcome them?

Note: * Scored in the reverse direction.

$[0] \quad[1] \quad[2] \quad[3] \quad[4]$

Used with permission of the publisher from Cohen S, Kamack T, Mermelstein R. A Global Measure of Perceived Stress. J Health and Social Behav 1983; 24: 385-396. 
APPENDIX E 


\section{Florida International University \\ Knowledge of Cardiovascular Risk Factors Questionnaire}

Dear Participant: Please circle True (T) or False (F) to the following questions to test your knowledge of heart disease and its risk factors.

Pin \#:

1 - T F The risk factors for heart disease that you can do something about are: high blood pressure, high blood cholesterol, smoking, obesity and physical inactivity.

2- T F

A stroke is often the first symptom of high blood pressure, and a heart attack is often the symptom of high blood cholesterol.

3- T F

A blood pressure greater than or equal to $140 / 90 \mathrm{~mm} \mathrm{Hg}$ is generally considered to be high.

4- T F

High blood pressure affects the same number of AfricanAmericans and Caribbean-Americans as it does non-Hispanic Whites.

$5-\mathrm{T}$ F

The best ways to treat and control high blood pressure are to control your weight, exercise, eat less salt (sodium), restrict your intake of alcohol, and take your high blood pressure medicine, if prescribed by your doctor.

$6-\mathrm{T} \quad \mathrm{F}$

A blood cholesterol level of $240 \mathrm{mg} / \mathrm{dL}$ is desirable for adults.

7- T F

The most effective dietary way to lower the level of your blood cholesterol is to eat food low in cholesterol.

8- T F

Lowering blood cholesterol levels can help people who have already had a heart attack.

9- $\mathrm{T}$ F

Only children from families at high risk of heart disease need to have their blood cholesterol levels checked.

$10-\mathrm{T} F$

Smoking is a major risk factor for 4 of the 5 leading causes of death including heart attack, stroke, cancer and lung diseases such as emphysema and bronchitis.

$11-\mathrm{T}$ F

If you have had a heart attack, quitting smoking can help reduce your chances of having a second attack.

12 - T F

Someone who has smoked for 30 to 40 years probably will not be able to quit smoking. 
The best way to lose weight is to increase physical activity and eat fewer calories.

14 - T F

Heart disease is the leading killer of men and women in the United States.

$15-\mathrm{T} F$

High blood cholesterol is one of the risk factors for heart disease that you can do something about.

16- T F

To lower your blood cholesterol level you must stop eating meat altogether.

17- T F

Any blood cholesterol level below $240 \mathrm{mg} / \mathrm{dL}$ is desirable for adults.

$18-\mathrm{T} \quad \mathrm{F}$

Fish oil supplements are recommended to lower blood cholesterol.

19- T F

To lower your blood cholesterol level you should eat less saturated fat, total fat and cholesterol and, lose weight if your are overweight.

$20-\mathrm{T} F$

Saturated fat raises your blood cholesterol level more than anything else in your diet.

$21-\mathrm{T} F$

All vegetable oils help lower blood cholesterol levels.

$22-\mathrm{T} F$

Lowering blood cholesterol levels can help people who have already had a heart attack.

23- T F

All children need to have their blood cholesterol levels checked.

$24-\mathrm{T} F$

Women do not need to worry about high blood cholesterol and heart disease.

25- $\mathrm{T} F$

Reading food labels can help you eat the heart healthy way.

National Heart, Lung and Blood Institute Publication, Nos. 93-2724 and 95-3794 
APPENDIX F 
RESPONDENT ID NUMBER



This form is about the foods you usually eat. It will take about 15 - 25 minutes to complete.

- Please answer each question as best you can. Estimate if you aren't sure.

- Use only a No. 2 pencil.

- Fill in the circles completely, and erase completely if you make any changes.

Please print your name in this box.

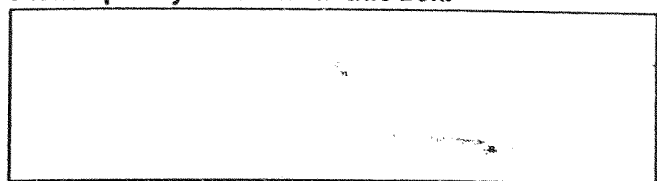

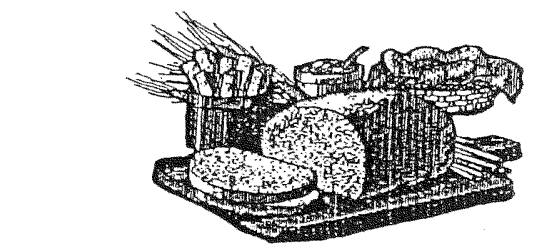

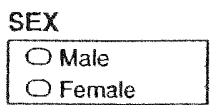

AGE

WEIGHT pounds
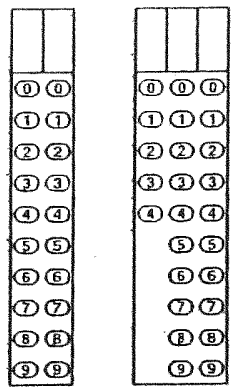

If Remale, are you pregnant or breast feeding?

\begin{tabular}{|l|}
\hline No \\
$\bigcirc$ Yes \\
$\bigcirc$ Not female \\
\hline
\end{tabular}

\begin{tabular}{|l|}
\hline \\
\hline (1) (0) \\
(1) (1) \\
(2) (2) \\
(3) (3) \\
(4) (4) \\
(5) (5) \\
(6) (6) \\
() (2) \\
(8) (B) \\
(2) (9)
\end{tabular}

HEIGHT

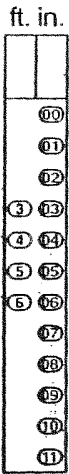

This form Is about your usual eating habits in the past year or so. This includes all meals or snacks, at home or in a restaurant or carry-out. There are two kinds of questions for each food.

HOW OFTEN, on average, did you eat the food during the past year? "Please DO NOT SKIP any foods. Mark "Never" if you didn't eat it.

HOW MUCH did you usually eat of the food?

"Sometimes we ask how many you eat, such as $1 \mathrm{egg}, 2$ eggs, etc., ON THE DAYS YOU EAT IT.

"Sometimes we ask "how much" as A, B, C or D. LOOK AT THE ENCLOSED PICTURES. For each food, pick the picture (bowls or plates) that looks the most like the serving size you usually eat. (If you don't have pictures: $A=1 / 4$ cup, $B=1 / 2$ cup, $C=1$ cup, $D=2$ cups.)

EXAMPLE: This person drank apple juice twice a week, and had one glass each time. Once a week he ate a "C"-sized serving of rice (about 1 cup).

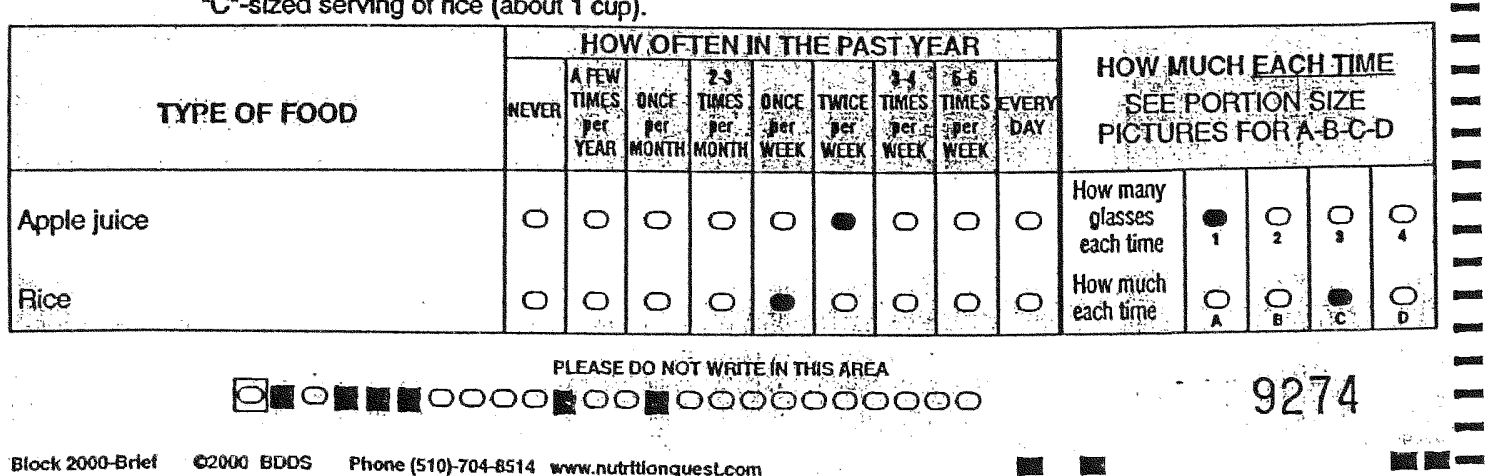




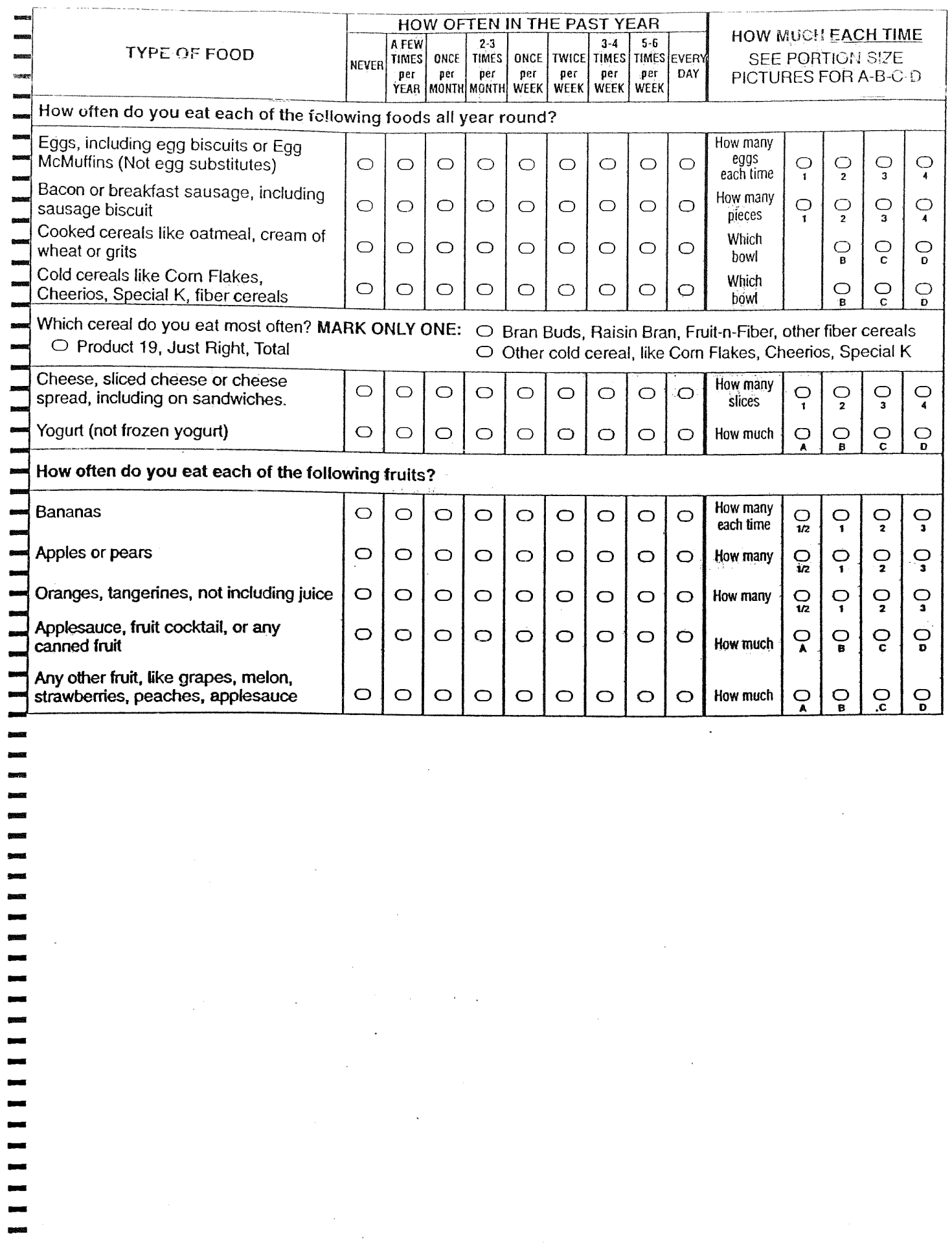




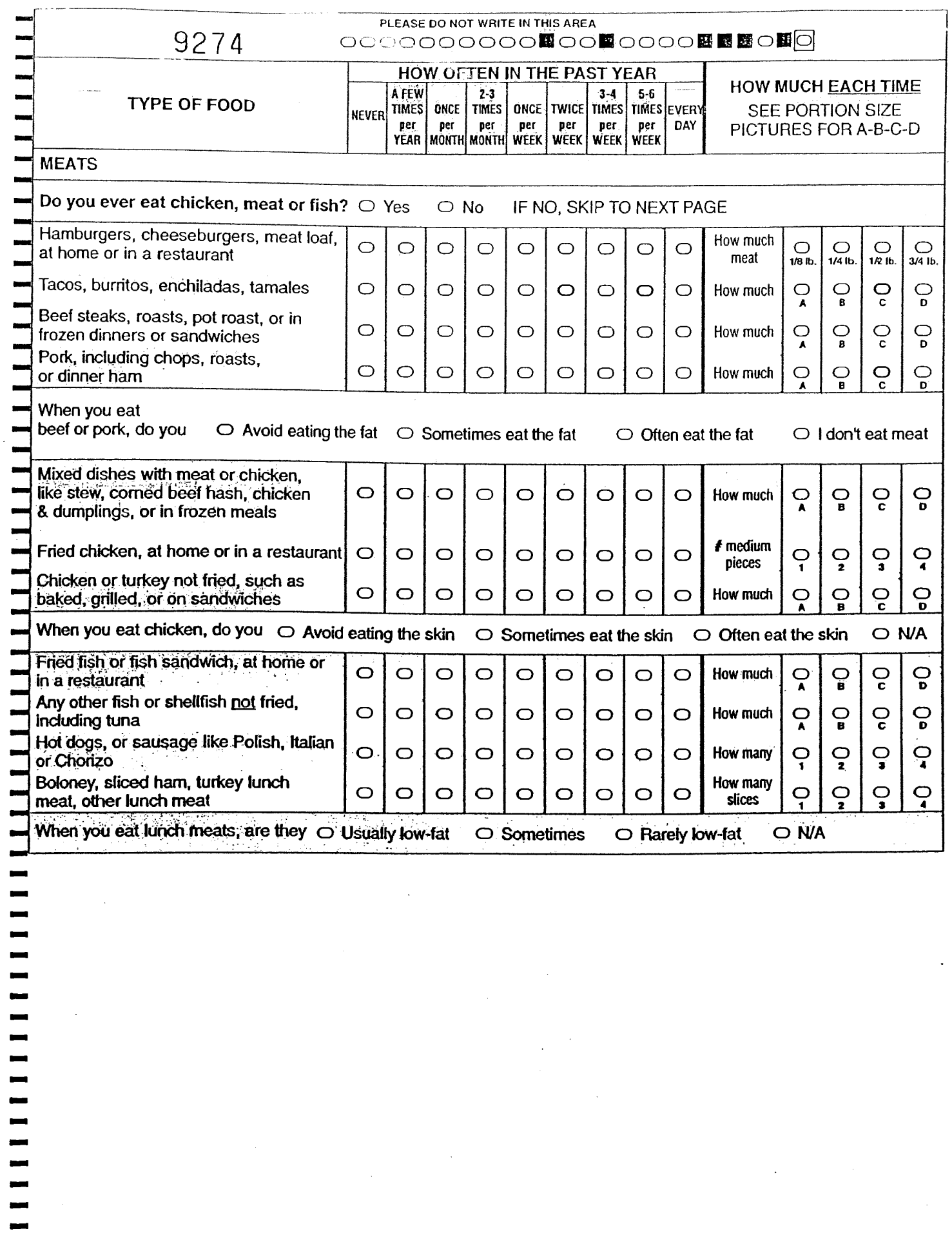




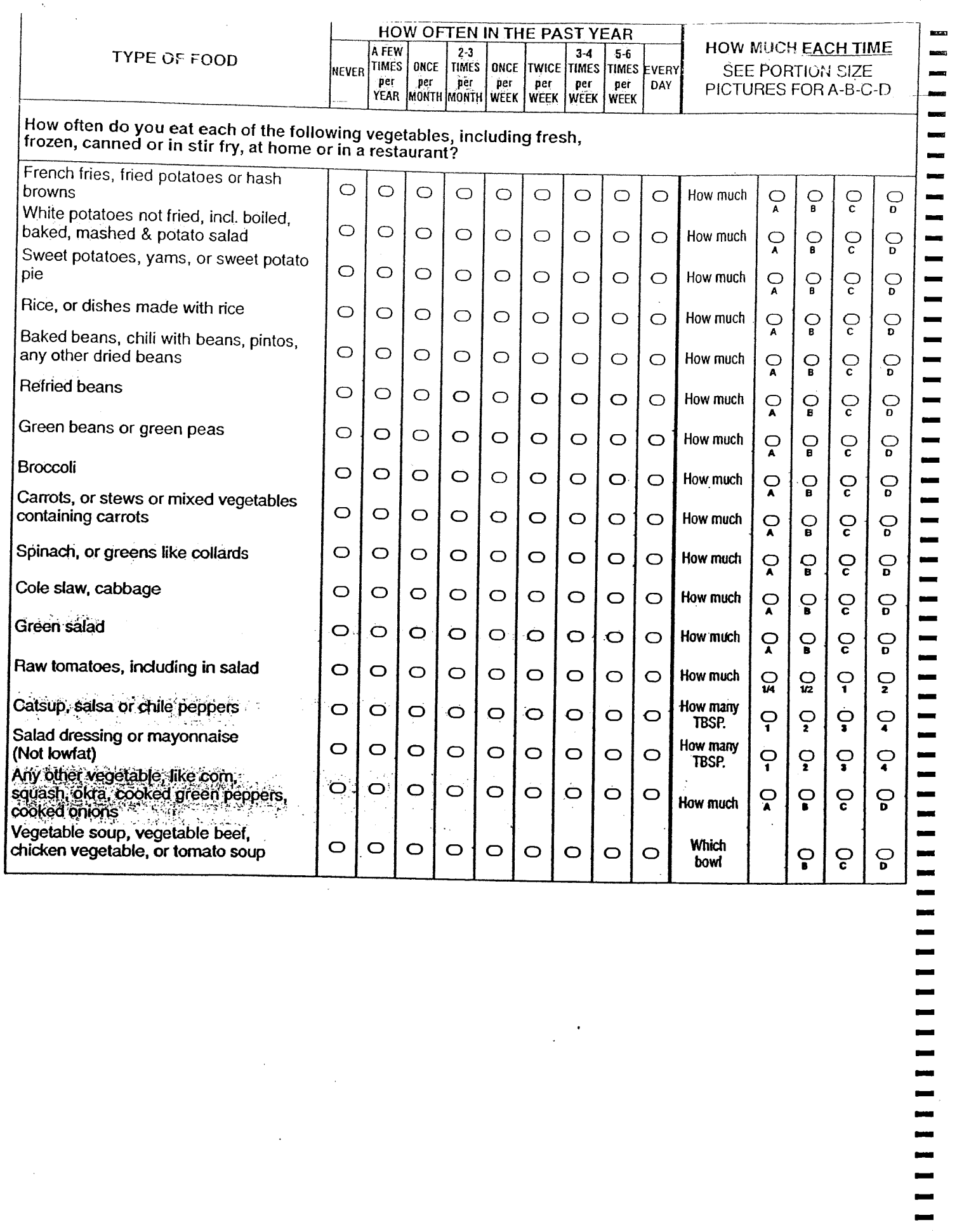




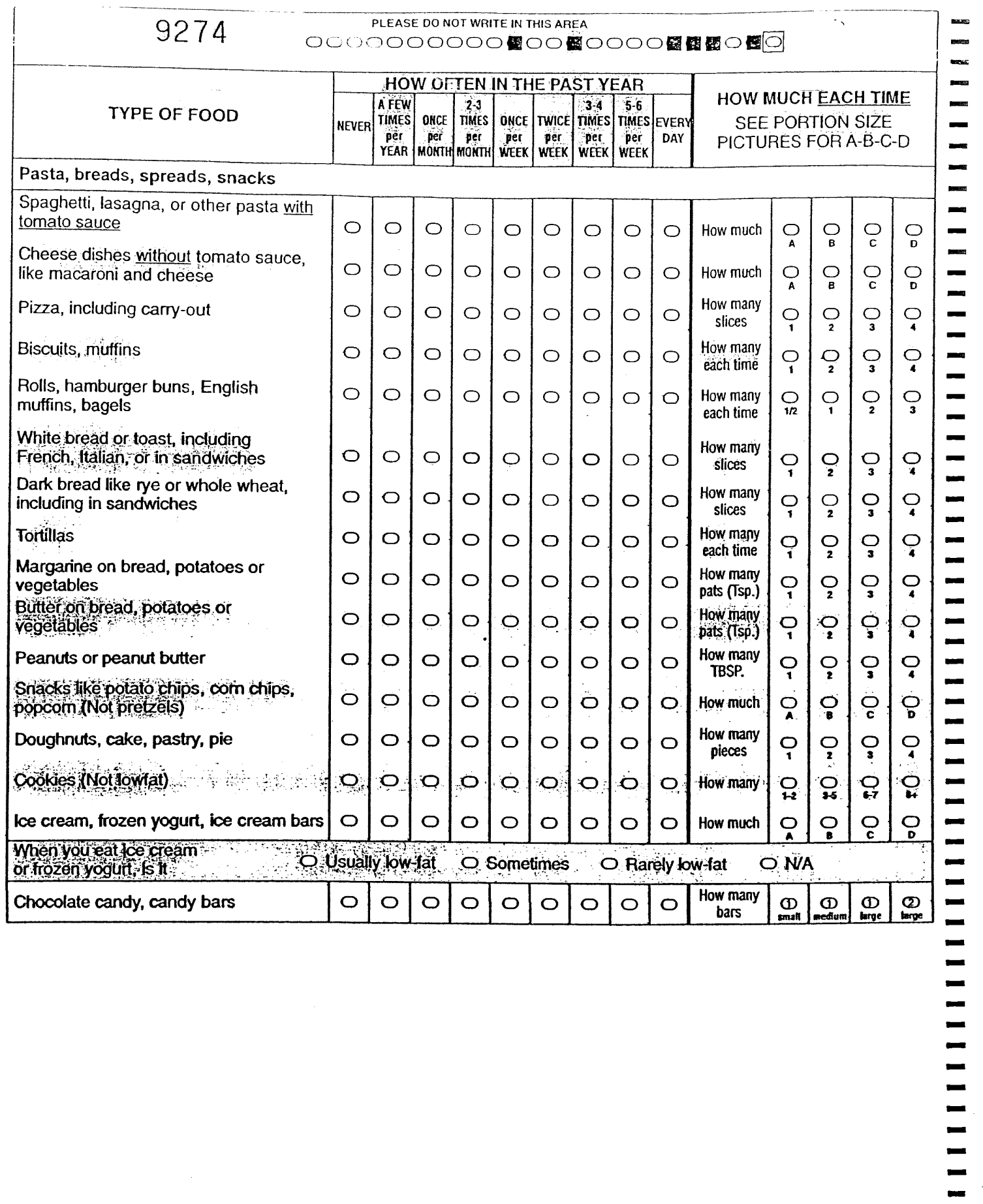




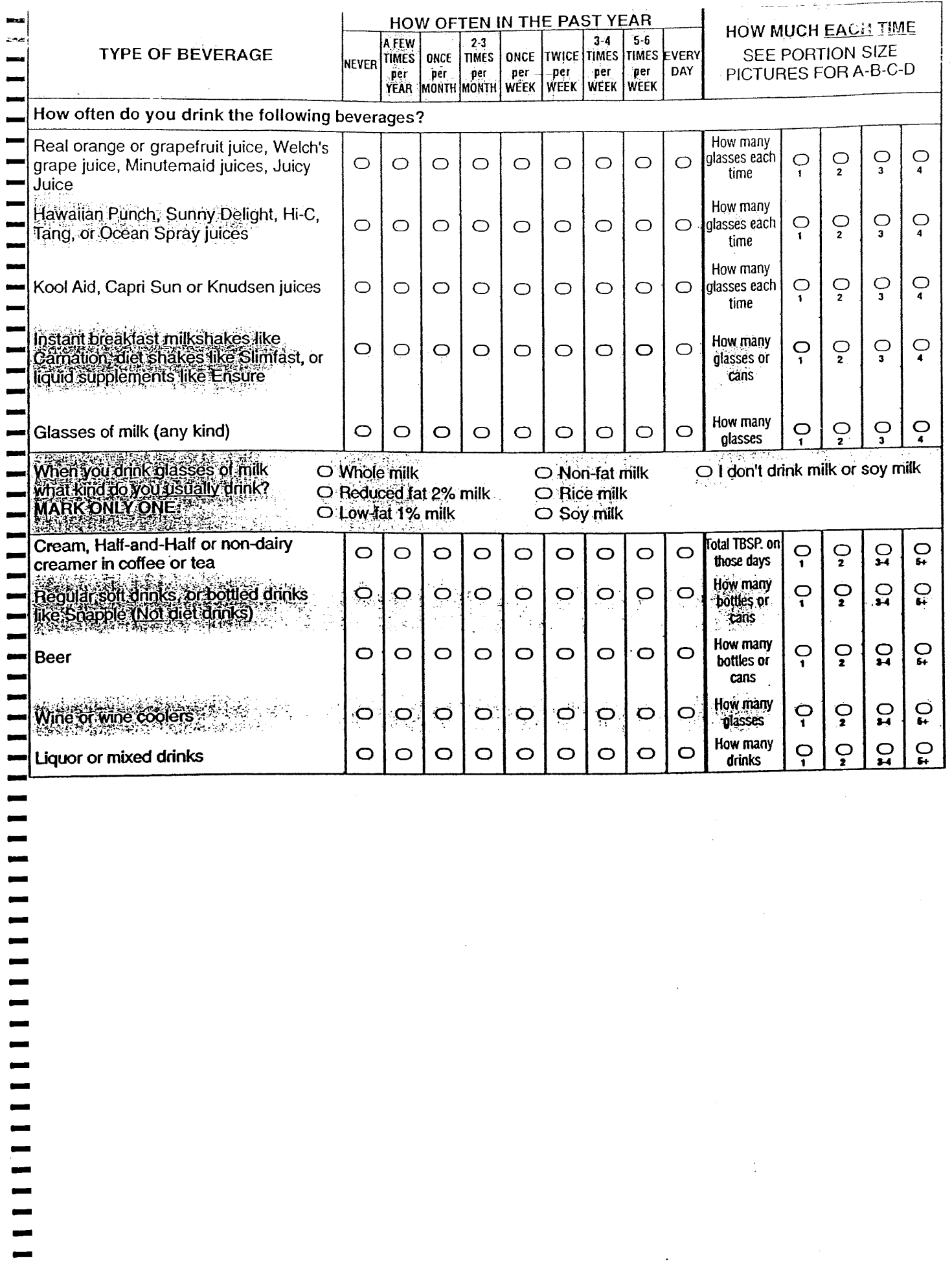


During the past year, have you taken ary witamins or minerals regularly, at least once a month?

$\bigcirc$ No, not regularly $O$ Yes, fairly regularly 2

(IF YES) WHAT DID YOU TAKE FAIRLY REGULARLY?

\begin{tabular}{|c|c|c|c|c|c|c|c|c|c|c|c|}
\hline \multirow[b]{3}{*}{ Multiple Vitamins. Did you take } & \multicolumn{5}{|c|}{ HOW OFTEN } & \multicolumn{6}{|c|}{ FOR HOW MANY YEARS? } \\
\hline & $\begin{array}{l}\text { DIDNT } \\
\text { TAKE }\end{array}$ & $\begin{array}{c}\text { A FEW } \\
\text { DAYS } \\
\text { per } \\
\text { MONTH } \\
\end{array}$ & \begin{tabular}{|c|}
$1-3$ \\
DAYS \\
per \\
WEEK \\
\end{tabular} & \begin{tabular}{|c|}
46 \\
DAYS \\
per \\
WEEK
\end{tabular} & $\begin{array}{c}\text { EVERY } \\
\text { DAY }\end{array}$ & $\begin{array}{l}\text { LESS } \\
\text { THAN } \\
\text { IYR. }\end{array}$ & YEAR & $\begin{array}{c}2 \\
\text { VEARS } \\
\end{array}$ & $\begin{array}{c}3-4 \\
\text { YEARS }\end{array}$ & $\begin{array}{c}5-9 \\
\text { YEARS }\end{array}$ & VEA \\
\hline & & & & & & & & & & & \\
\hline Regular Once-A-Day, Centrum, or Thera type & $\bigcirc$ & 0 & 0 & 0 & 0 & 0 & 0 & 0 & 0 & 0 & 0 \\
\hline Stress-tabs or B-Complex type & $\bigcirc$ & 0 & ○ & 0 & 0 & 0 & 0 & 0 & 0 & 0 & 0 \\
\hline $\begin{array}{l}\text { Antioxidant combination type } \\
\text { Sinqle Vitamins (not }\end{array}$ & O & 0 & 0 & ○ & 0 & 0 & 0 & 0 & 0 & O & 0 \\
\hline $\begin{array}{l}\text { Single Vitamins (not part of } \\
\text { Vitamin A (not beta-carote }\end{array}$ & & & & & & & & & & & \\
\hline Beta-carotene & 0 & 0 & 0 & $\odot$ & 0 & $\odot$ & 0 & 0 & 0 & 0 & 0 \\
\hline Vitamin C & O & 0 & 0 & 0 & 0 & 0 & 0 & 0 & 0 & 0 & 0 \\
\hline Vitamin E & O & 0 & 0 & 0 & 0 & 0 & 0 & 0 & 0 & 0 & ○ \\
\hline Folic acid, folate & $\bigcirc$ & 0 & 0 & 0 & 0 & 01 & 0 & 0 & 0 & 0 & 0 \\
\hline Calcium or Tums, alone or combined with vit. D or & 0 & 0 & 0 & 0 & 0 & 0 & 0 & 0 & 0 & 0 & 0 \\
\hline mag & $\bigcirc$ & 0 & 0 & 0 & ○ & 0 & 0 & 0 & O & 0 & 0 \\
\hline Zinc & 0 & 0 & 0 & 0 & $\bigcirc$ & 0 & 0 & రి & 0 & 0 & 0 \\
\hline Iron & 0 & 0 & 0 & 0 & 0 & 0 & 0 & 0 & 0 & 0 & 0 \\
\hline Selenium & 0 & 0 & 0 & 0 & 0 & 0 & 0 & 0 & 0 & $\varnothing$ & 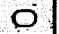 \\
\hline Vitamin $D_{1}$ alone or combined with & 0 & 0 & 0 & 0 & 0 & 0 & 0 & 0 & 0 & 0 & 0 \\
\hline
\end{tabular}

If you took vitamin $\mathrm{C}$ or vitamin $\mathrm{E}$ :

How many milligrams of vitamin $\mathrm{C}$ did you usually take, on the days you took it?

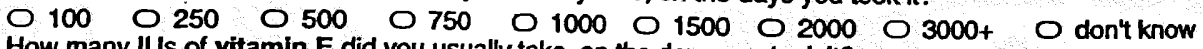
How many IUs of vitamin E did you usually take, on the days you took it?
O 100
0200
$0300 \bigcirc 400$
0600
800
01000
$02000+\quad 0$ don't know

How often do you use fat or oil In cooking?

$O$ Less than once per week OA few times per week O Once a day $O$ Twice a day $O 3+$ per day

What kinds of fat or oll do you usually use in cooking? MARK ONLY ONE OR TWO

O Don't know, or Pam O Butter/margarine blend O Lard fattock,

O Stick margarine

O Low-fat margarine

Soft tub margarine

Com oil, vegetable oil

O Butter

$\checkmark$ Olive oil or canola oil

Crisco

Did you ever drink more beer, wine or liquor than you do now? O Yes O No

Do you smoke cigarettes now? O Yes $O$ No

IF YES, On the average about how many cigarettes a day do you smoke now?

O 1-5 O 6-14 O 15-24 O 25-34 O 35 or more

What is your ethnic group? (MARK ONE OR MORE)

Hispanic or Latino O Black or African American O American Indian or Alaska Native

White, not Hispanic $\mathrm{O}$ Asian

O Native Hawaiian or Other Pacific Islander

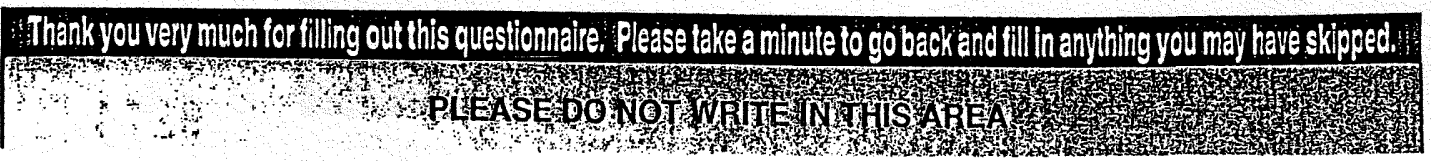



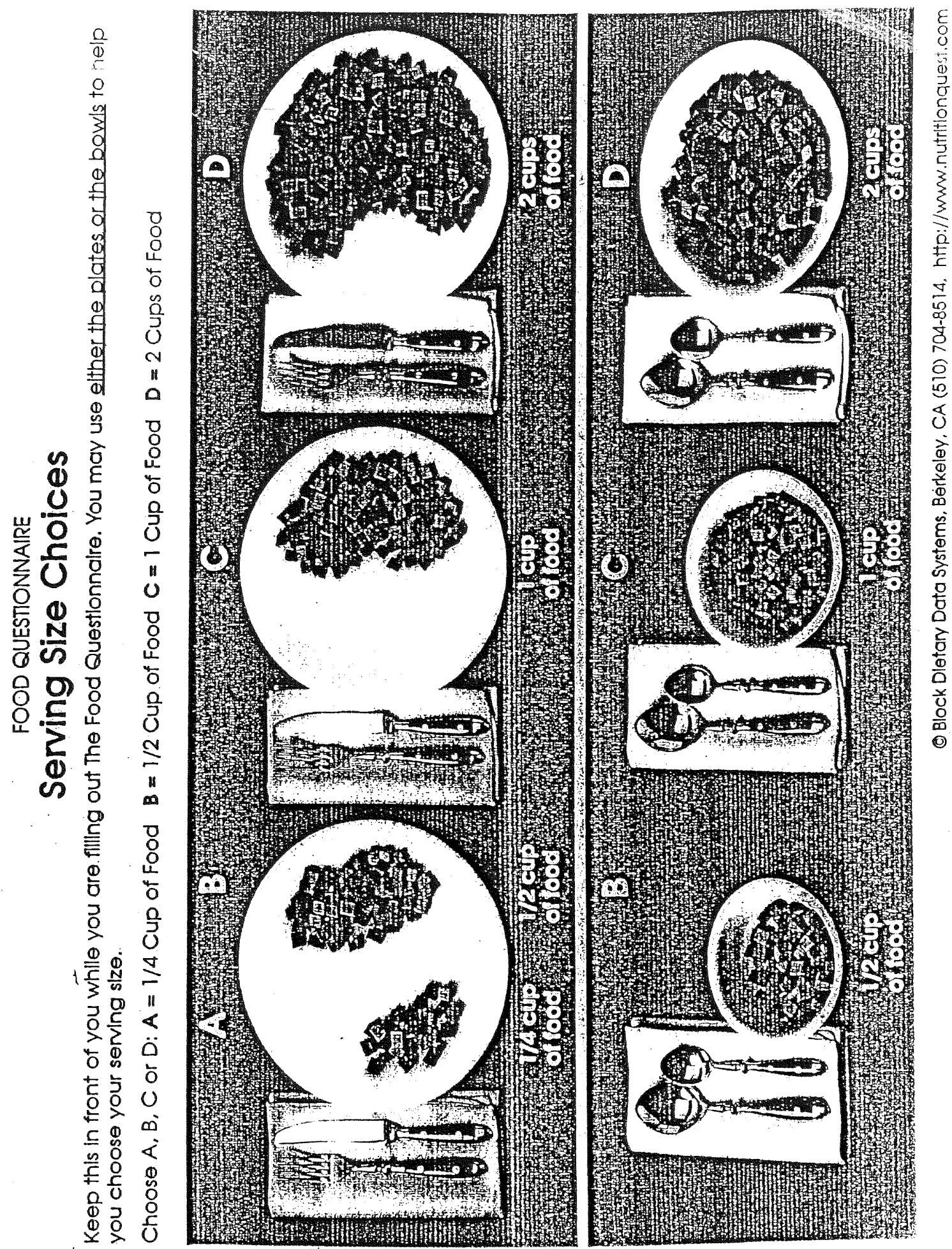
Please think. Are there any foods you did not see on this questionnaire which you ate at least once per week, even in small quantities, or that you ate frequently in a particular season. Please indicate in the spaces below. Thank you.

How Many Times a Day, Week or a Month Would You Say You Ate the Particular Food Item? Please See Portion Size Pictures for A-B-C-D.

\begin{tabular}{|c|c|c|c|c|c|c|c|}
\hline \multirow[t]{2}{*}{ Food } & \multicolumn{4}{|c|}{ Serving Size } & \multicolumn{3}{|c|}{ How Often } \\
\hline & $\mathrm{A}$ & $\mathrm{B}$ & $\mathrm{C}$ & $\mathrm{D}$ & Day & Week & Month \\
\hline & & & & & & & \\
\hline & & & & & & & \\
\hline & & & & & & & \\
\hline & & & & & & & \\
\hline & & & & & & & \\
\hline & & & & & & & \\
\hline & & & & & & & \\
\hline & & & & & & & \\
\hline & & & & & & & \\
\hline & & & & & & & \\
\hline & & & & & & & \\
\hline & & & & & & & \\
\hline & & & & & & & \\
\hline & & & & & & & \\
\hline & & & & & & & \\
\hline & & & & & & & \\
\hline & & & & & & & \\
\hline & & & & & & & \\
\hline & & & & & & & \\
\hline & & & & & & & \\
\hline & & & & & & & \\
\hline & & & & & & & \\
\hline & & & & & & & \\
\hline & & & & & & & \\
\hline & & & & & & & \\
\hline & & & & & & & \\
\hline & & & & & & & \\
\hline & & & & & & & \\
\hline & & & & & & & \\
\hline & & & & & & & \\
\hline & & & & & & & \\
\hline & & & & & & & \\
\hline & & & & & & & \\
\hline & & & & & & & \\
\hline & & & & & & & \\
\hline
\end{tabular}


APPENDIX G 


\section{Baecke Questionnaire of Habitual Physical Activity}

\section{Please circle or write the appropriate response}

1. What is your main occupation? $1-3-5$

2. At work, I sit (never/seldom/sometimes/often/always) $1-2-3-4-5$

3. At work, I stand (never/seldom/sometimes/often/always) $1-2-3-4-5$

4. At work, I walk (never/seldom/sometimes/often/always) $1-2-3-4-5$

5. At work, I lift heavy loads (never/seldom/sometimes/often/very often) $1-2-3-4-5$

6. After work, I am tired (very often/often/sometimes/seldom/never) _ 5-4-3-2-1

7. At work, I sweat (very often/often/sometimes/seldom/never) $5-4-3-2-1$

8. In comparison with others my own age, I think my work is physically much heavier/heavier/as heavy/lighter/much lighter $5-4-3-2-1$

9. Do you play sport? Yes/No

If yes:

- which sport do you play most frequently? Intensity $0.76-1.26-1.76$

- How much hours per week? $<1 / 1-2 / 2-3 / 3-4 />4$ Time $0.5-1.5-2.5-3.5-4.5$

- How many months per year? $<1 / 1-3 / 4-6 / 7-9 />9$ Proportion $0.04-0.17-0.42-0.67-0.92$

If you play a second sport:

- Which sport do you play most frequently? _ Intensity $0.76-1.26-1.76$

- How much hours per week? $<1 / 1-2 / 2-3 / 3-4 />4$ Time $0.5-1.5-2.5-3.5-4.5$

- How many months per year? _ $<1 / 1-3 / 4-6 / 7-9 />9$

Proportion $0.04-0.17-0.42-0.67-0.92$

10. In comparison with others my own age, I think my physical activity during leisure is much more/more/the same/less/much less $5-4-3-2-1$

11. During leisure time, I sweat very often/often/sometimes/seldom/never $5-4-3-2-1$

12. During leisure time, I play sport never/seldom/sometimes/often/very often $1-2-3-4-5$

13. During leisure time, I watch television never/seldom/sometimes/often/very often $1-2-3-4-5$ 
14. During leisure time

I walk never/seldom/sometimes/often/very often $1-2-3-4-5$

15. During leisure, I cycle never/seldom/sometimes/often/very often $1-2-3-4-5$

16. How many minutes do you walk and/or cycle per day to and from work, school, and shopping? $<5 / 5-15 / 15-30 / 30-45 />45$ $1-2-3-4-5$ 


\section{INSTRUCTIONS}

The Baecke Questionnaire is self-explanatory.

\section{CALCULATIONS}

Scoring of the questionnaire was describe by Baecke et al (1982) as follows:

Work activity index (WAI) = mean score from the occupational Likert scale questions 1-8.

Note: Score categories for question 1 are:

$1=$ 'Low level' occupations such as office or clerical work, driving, teaching or studying

$2=$ 'Middle level' occupations such as factory work, plumbing or carpentry

$3=$ 'High level' occupations such as dock work or construction work.

Sports activity index $(\mathrm{SAI})=$ mean score of questions $9-12$.

Note: Score for question $9=$ sum of (proportion of year of participation $\times$ intensity code $\times$ time [duration] for all activities. The intensity, duration and proportion codes can be found on the questionnaire.

'Low level sport' = billiards, sailing, bowling, golf.

'Middle level sport' = badminton, cycling, dancing, swimming, tennis.

'High level sport' = boxing, basketball, football, rugby, rowing.

Non-sports leisure-time activity index (LTAI) =mean score of questions 13-16.

Total score $=\mathrm{WAI}+\mathrm{SAI}+\mathrm{LTAI}$.

Note: For questions 2 and 13, the Likert scale response is subtracted from six. Since the questionnaire scores have no inherent unit of measure (e.g., kcal/unit time), they were designed to be categorized into quartiles for general classification of activity levels.

\section{EXAMPLE}

Hypothetical responses to the Baecke Questionnaire (Question [Response]: 1 (3); 2 (4); 3 (3); 4 (5); 5 (2); 6 (2); 7 (1); 8 (3); 9 (cycling, 3-4 hours/week, 4-6 months/year and, volleyball, 1-2 hours/week, 1 -3 months/year); 10 (4); 11 (4); 12 (4); 13 (3); 14 (3); 15 (5); 16 (1):

WAI (questions $1-8)=(3+[6-4]+3+4+2+2+1+3) \div 8=2.5$

SAI (questions 9-12) $=\left(2^{*}+4+4+4\right) \div 4=3.5$

- *Calculation for $9:(0.42 \times 1.76 \times 3.5)+(0.17 \times 1.26 \times 1.5)=2.9$

- The value of 2.9 is scored as a 2.0 according to the following scale:

- 0 (no sport reported) $=1 ; 0.01<4=2 ; 4<8=3 ; 8<12=4 ; \geq 12=5$.

LTAI (questions $13-16)=([6-3]+3+5+1) \div 4=3.0$

Total score $=2.5+3.5+3.0=9.0$

Used with permission of the publisher from Baecke JA, Burma J, Frijters JER. A Short Questionnaire for the Measurement of Habitual Physical Activity in Epidemiological Studies. Am J Clin Nutr 1982; 36: 936-942. 
APPENDIX H 
Pin \#

$\begin{array}{ll}\text { Weight } & \text { lb } \\ \text { Weight } & \mathrm{kg}\end{array}$

Height _ in

Height $\mathrm{cm}$ BMI

Waist circumference

in Waist circumference cm

Blood Pressure: 1

Systolic

Diastolic $\mathrm{mm} \mathrm{Hg}$ $\mathrm{mm} \mathrm{Hg}$
Blood Pressure: 2

Systolic

Diastolic $\mathrm{mm} \mathrm{Hg}$ Average $\mathrm{mm}$ Hg Average $\mathrm{mm} \mathrm{Hg}$ $\mathrm{mm} \mathrm{Hg}$

Blood Lipids

Total Cholesterol $\mathbf{m g} / \mathbf{d L}$

High-density Lipoprotein Cholesterol $\mathrm{mg} / \mathrm{dL}$

Triglyceride $\mathrm{mg} / \mathrm{dL}$

Low-density Lipoprotein Cholesterol $\mathrm{mg} / \mathrm{dL}$

Blood Glucose $\mathrm{mg} / \mathrm{dL}$

High-sensitivity C-reactive Protein $\mathrm{mg} / \mathrm{L}$

Cholesterol/HDL-C

VLDL $\mathbf{m g} / \mathbf{d L}$ 


\section{APPENDIX I}




\section{Glossary of Health and Cultural Terms}

Acculturation: As a group that stays in the (United StatesUS for a period of time, they become more like the dominant culture because of changes that occur due to the repeated exposure to influence from another culture. The extent of this transformation is related to age, education and opportunities for interaction with another culture. Individuals within groups will be found to lie all along the continuum representing various levels of change.

Age-adjusted Rates: Used mainly to compare the rates of two or more communities, population groups or the country as a whole, over time. A standard population year (2000) is used so that these rates are not affected by changes or differences in the age composition of the population.

Angina (angina pectoris): A pain or discomfort in the chest that occurs when some part of the heart does not receive enough blood. Angina is a common symptom of coronary heart disease.

Atherosclerosis: Hardening of the arteries in which cholesterol and other substances in the blood are deposited in the walls of arteries, including coronary arteries that supply blood to the heart.

Bias: Any systematic error in the design, conduct or analysis of a study that results in a mistaken estimate of an exposure's effect on the risk of disease.

Body Mass Index (BMI): A mathematical formula to assess body weight relative to height. This measure correlates highly with body fat. It is calculates as weight in kilograms divided by the square of the height in meters $\left(\mathrm{kg} / \mathrm{m}^{2}\right)$. Because it is easily calculated, BMI is the measurement of choice as an indicator of healthy weight, overweight and obesity.

Blood Pressure: The force of blood pushing against the walls of the arteries. Blood pressures less than 120/80 are considered normal.

Cardiovascular Disease (CVD): Includes a variety of diseases of the heart and blood vessels, coronary heart disease (coronary artery disease, ischemic heart disease), stroke (brain attack), high blood pressure (hypertension), rheumatic heart disease, congestive heart failure and peripheral artery disease (ICD/10 codes 100-199, Q20-Q28).

\section{Centers for Disease Control and Prevention/National Center for Health Statistics} (CDC/NCHS): A division of the US Department of Health and Human Services (USDHHS). The CDC conducts the Behavioral Risk Factor Surveillance System (BRFSS, ongoing). The NCHS conducted the National Health Examination Survey (NHES) and the National Health and Nutrition Examination Surveys (NHANES) I (197174), II (1976-80) \& III (1988-94). The NCHS also conducts these ongoing studies (among others): The National Health Interview Survey (NHIS), The National Home and 
Hospice Care Survey, The National Hospital Ambulatory Medical Care Survey and The national Hospital Discharge Survey.

Coronary Heart Disease (CHD): A condition in which the flow of blood to the heart muscle is reduced. If enough oxygen-carrying blood cannot reach the heart muscle, one will feel pain in the chest or sometimes in the left arm or shoulder (angina). When the blood supply is cut off completely, the result is a heart attack. This includes ICD/10 codes 120-125.

Culture: The values, norms, traditions, customs, arts, history folklore and institutions that are developed, learned and shared by a group of people and passed down from one generation to the next. Culture is not static. It is an ongoing process within which individuals are constantly trying out new ideas and behaviors to better meet their goals and objectives. Culture is not monolithic. People within a racial/ethnic group are heterogenous. Put another way culture is a group which shared experiences, language and values that permit people to communicate in ways not shared by those outside the culture. Under certain circumstances, such a group may go beyond racial or ethnic categories.

Culturally Appropriate: An unbiased attitude and organizational policy that values cultural diversity in the population served. Reflects an understanding of diverse attitudes, beliefs, behaviors, practices, habits and communication patterns that could be attributed to age, gender, race, ethnicity, religion, socio-economic status, physical or mental ability, or generational and acculturational status. It includes an awareness that cultural differences may affect health, health outcomes and the effectiveness of health care delivery.

Cultural Sensitivity: The recognition that cultural differences as well as similarities exist, that both are valued, and there is no right or wrong cultural difference. They are just different.

Death Rate: Sometimes reported as "crude" death rate reflects the frequency with which death occurs within some specified interval of time in a population. However, agespecific or sex-specific rates are more meaningful and informative. This is because it allows one to look at well-defined sub-groups of the total population.

Ethnicity: Belonging to a common group, often linked by race, nationality and language with a common cultural heritage or derivation. Ethnicity is also a construct that encompasses both genetic and cultural differences.

Health: A state of physical, mental and social well-being and merely the absence of disease and infirmity.

Health Outcomes: The results or consequences of a process of care. Health outcomes may include satisfaction with care as well as the use of health care resources. This 
includes clinical outcomes such as changes in health status and changes in the length and quality of life as a result of detecting or treating disease.

Health Literacy: The degree to which individuals have the capacity to obtain, process and understand basic health information and services needed to make appropriate healthdecisions.

Health Promotion: Any planned combination of educational, political, regulatory and organizational supports for actions and conditions of living conducive to the health of individuals, groups and communities.

Incidence: An estimate of the number of new cases of a disease that develop in a population in a one-year period.

Morbidity: Incidence and prevalence rates are both measures of morbidity, that is, measures of various effects of disease on a population.

Mortality: The total number of deaths from a given disease in a population during a specific interval of time, usually a year.

National Heart, Lung and Blood Institute (NHLBI): An institute in the National Institutes of Health in the USDHHS. The NHLBI conducts the Atherosclerosis Risk in Communities (ARIC) study (ongoing since 1985), The Cardiovascular Health Study (CHS, ongoing since 1988), The Framingham Heart Study (FHS, ongoing since 1948), The Honolulu Heart Program (HHP, 1965-97) and the Strong Heart Study (SHS, 198992; 1991-98). The NHLBI publishes the reports of the Joint National Committee on Prevention, Detection, Evaluation and Treatment of High Blood Pressure, JNC VII, the most recent.

Prevalence: An estimate of the total number of cases of a disease existing in a population at a specific point in time. Prevalence is sometimes expressed as a percentage of population.

Primary Prevention: Health care services, medical tests, counseling and health education and other actions designed to prevent the onset of a targeted condition. Routine immunization of healthy individuals is an example of primary prevention.

Race: A socially defined population that is derived from distinguishable physical characteristics that are genetically transmitted.

Risk Factor: A characteristic that has been demonstrated statistically to be associated with a particular injury.

Secondary Prevention: Measures such as health care services designed to identify or treat individuals who have a disease or risk factors of a disease but who are yet to 
experience symptoms of the disease. Cholesterol and high blood pressure screenings are examples of secondary prevention.

Target Population: The group of individuals (usually those at high risk) whom program interventions are designed to reach.

Tertiary Prevention: Preventive health care services that are part of the treatment and management of individuals with clinical illness. Example of tertiary prevention include cholesterol reduction in persons with CHD and insulin therapy to prevent complications of diabetes mellitus.

The definitions were adapted from:

American Heart Association. 2004 Heart and Stroke Statistical Update. Dallas, TX.

Healthy People 2010. With Understanding and Improving Health and Objectives for Improving Health. (November 2000) $\left(1^{\text {st }}\right.$ and $2^{\text {nd }}$ ed) Washington, DC: US Government Printing Office, US Department of Health and Human Services.

Healthy People 2010. Heart Disease and Stroke. (November 2000) $\left(1^{\text {st }}\right.$ ed) Washington, DC: US Government Printing Office, US Department of Health and Human Services.

Bronner Y. Cultural Sensitivity and Nutrition Counseling. Topics in Clinical Nutrition 1994; 9(2): 13-19.

Anand SS. Using Ethnicity as a Clssification Variable in Health Research: Perpetuating the

Myth of Biological Determinism, Serving Socio-political Agendas, or Making Valuable Contributions to Medical Sciences? Ethn Health 1999; 4(4): 241-244.

Susuf S, Reddy S, Ounpuu S, Anand S. Global Burden of Cardiovascular Diseases Part 1: General Considerations, the Epidemiologic Transition, Risk Factors, and Impact of Urbanization. Circulation 2001; 104: 2746-2753.

Maitland, TE. The Health and Nutritional Status of Multiethnic Perimenopausal Women. $\mathrm{Ph} . \mathrm{D}$ Thesis, Florida International University, 2001. 


\section{VITA}

EDUCATION:

2001

1996

1993

1986

1978

\section{EXPERIENCE:}

\section{ERROL E. DAVIS \\ Born, Jamaica, West Indies}

Graduate Teaching Certificate

The Academy for the Art of Teaching

Florida International University, Miami

Certificate, Principles and Techniques of Social Marketing and Counseling

Pan American Health Organization/Caribbean Food and Nutrition Institute (PAHO/CFNI)

Certificate, Spa Cuisine

Caribbean Hospitality Training Institute

Kingston, Jamaica

M.Sc., Nutrition

Tropical Metabolism Research Unit (TMRU)

University of the West Indies, Kingston, Jamaica

B.Sc., Natural Sciences ( $2^{\text {nd }}$ Class Honors)

University of the West Indies

Mona, Kingston, Jamaica
$2003-2004$
$1999-2004$
1997- 1998
$1988-1998$
Research Assistant
Health Science Recruitment and Retention Program
Florida International University, Miami
Teaching Assistant/Adjunct Professor
Florida International University
Miami, Florida
Nutrition Consultant
Pan American Health Organization/Caribbean Food and Nutrition Institute (PAHO/CFNI)
University of the West Indies, Jamaica
External Examiner in Hospitality \& Food Science
Department at College of Arts Science and
Technology, Kingston, Jamaica 
1983-1984

Nutritionist and Research Assistant at the Ministry of Health (Portland) and CFNI, University of the West Indies, Kingston, Jamaica

\section{AWARDS/HONORS:}
$2002-2004$
Latin American and Caribbean Scholarship
1999 - Present
Department of Dietetics and Nutrition Graduate
Assistantship Award
2003
Florida International University Foundation Grant
2003
Graduate Student Association Research Award
2003
Wolmer's Alumni Association, South Florida
Chapter Scholarship
2001
Graduate Student Association Scholarly
Award Florida International University

\section{PUBLICATION:}

Davis E, Nath S, Huffman F, Dixon Z. Coronary Heart Disease Risk among Foreign- and US-born Individuals of African Ancestry. Federation of American Societies for Experimental Biology. FASEB J 2004; 18(4): A 147.

Davis E, Huffman F, Nath S. In Black Women HDL Cholesterol is Associated with Body Mass Index and Central Obesity. Federation of American Societies for Experimental Biology. FASEB J 2004; 18(5): A857.

Davis EE. Sports Nutrition. Cajanus Quarterly 1996;29(1).

Davis EE. Sports Nutrition. Candi J 1994; 1(1): 39-43.

Davis EE, Hibbert, JM, Jackson AA. The relationship between Glutathione and Fatty Infiltration of the Liver in Weanling Rats. Nutrition Reports International 1988; 37 (4): 847-856.

Davis EE, Hibbert JM, Jackson AA. The relationship between Glutathione and Fatty Infiltration of the Liver in Weanling Rats. W I Med J 1985; 34(suppl): 36. 